\title{
A NOVEL AND DYNAMIC PREDICTION ENGINE FOR PRACTICING PRECISION MEDICINE TO PREVENT CHEMOTHERAPY- INDUCED NAUSEA AND VOMITING
}

A Dissertation presented to

the Faculty of the Graduate School

at the University of Missouri

In Partial Fulfillment

Of the Requirements for the Degree

Doctor of Philosophy

By

ABU SALEH MOHAMMAD MOSA

Dr. Illhoi Yoo, Dissertation Supervisor

DECEMBER 2015 
(C) Copyright by ABU SALEH MOHAMMAD MOSA All Rights Reserved 
The undersigned appointed by the Dean of the Graduate School, have examined the dissertation entitled:

\section{A NOVEL AND DYNAMIC PREDICTION ENGINE FOR PRACTICING PRECISION MEDICINE TO PREVENT CHEMOTHERAPY-INDUCED NAUSEA AND VOMITING}

presented by Abu Saleh Mohammad Mosa,

a candidate for the degree of Doctor of Philosophy and hereby certify that, in their opinion, it is worthy of acceptance.

Dr. Illhoi Yoo

Dr. A. Mosharraf Hossain

Dr. Suzanne A. Boren

Dr. Mihail Popescu

Dr. Jerry C. Parker 


\section{DEDICATION}

To my loving mother, Meherneka Begum, who is by far the strongest women I have even known, who has been very caring, and who has been very keen for my education regardless of any obstacles.

To the memory of my loving father, Md. Abul Bashar, who is my role model, who raised me as a righteous and hard-working person, and whose last words are always encouraging to me. He inspired me to achieve the goals of my life regardless of any obstacles.

To my loving wife, Sadia Akter, who has shared tremendous amount of sacrifices for completing this dissertation but always been very proud and supportive of my work.

To my loving son, Ahnaf Mosa, who has born to be the bravest man in this world and missed the precious moments of his first two years of childhood. 


\section{ACKNOWLEDGEMENTS}

This project would not have been possible without the support of many people. Firstly, I would like to thank my adviser, Dr. Illhoi Yoo. His guidance, encouragements, and continuous support over the duration of my research have been invaluable, without which, the completion of this dissertation would not have been possible. Secondly, I would like to thank Dr. A. Mosharraf Hossain, who has clinical specialization in Cancer treatment. His guidance and help for data collection and research design have been invaluable. I am also thankful to Ellis Fischel Cancer Center for supporting the project.

Also thanks to my committee members, Dr. Jerry C. Parker, Dr. Suzanne A. Boren, and Dr. Mihail Popescu, who offered guidance and support. I am also thankful to the people at the MU Informatics Institute, Dr. Chi-Ren Shyu, previous DGS Dr. Sanda Erdelez, current DGS Dr. Gavin Conant, Robert Sanders and Tracy Pickens. Their advice and help with administrative matters were instrumental to my progress.

And finally, thanks to my parents, my wife Sadia Akter, and numerous friends who endured this long process with me, always offering much needed support, encouragement and love. 


\section{TABLE OF CONTENTS}

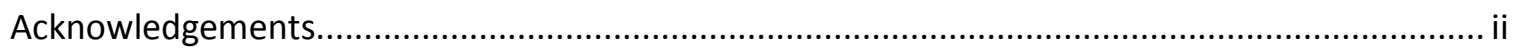

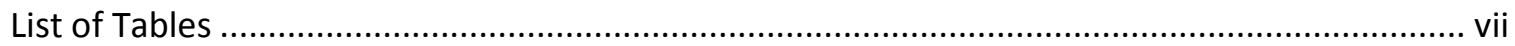

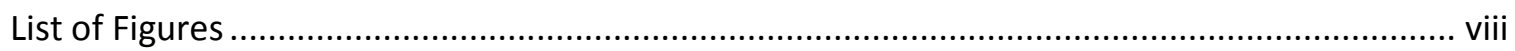

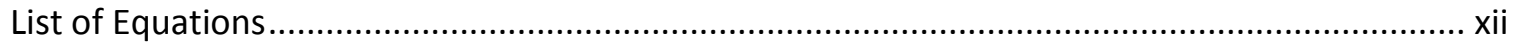

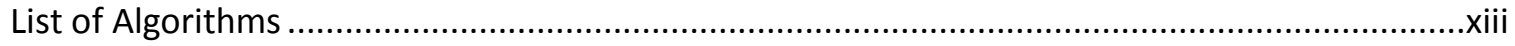

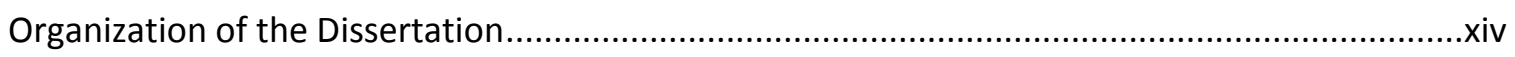

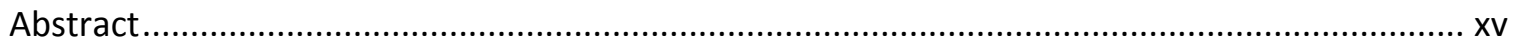

Chapter 1 : Chemotherapy Induced Nausea and Vomiting (CINV) .......................................... 1

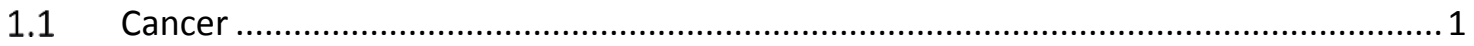

1.2 Chemotherapy for Cancer Treatment................................................................... 2

1.3 Chemotherapy Induced Nausea and Vomiting ............................................................

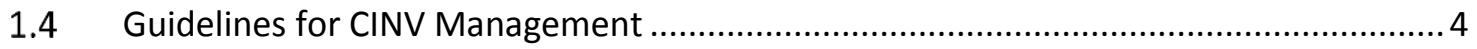

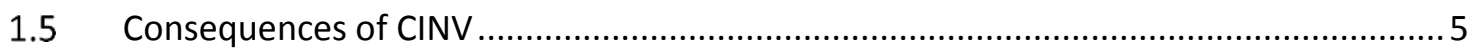

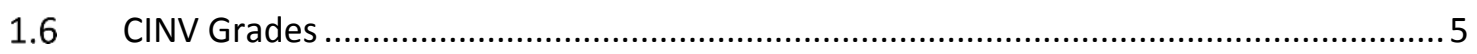

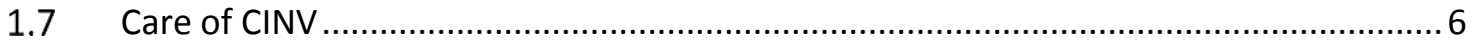

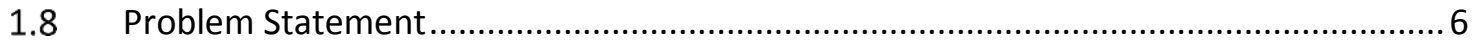

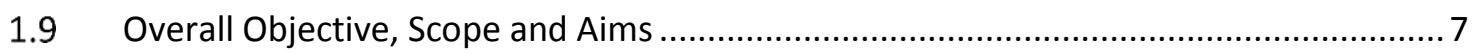

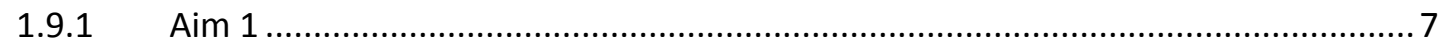

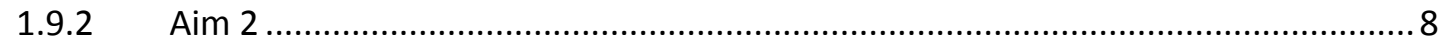

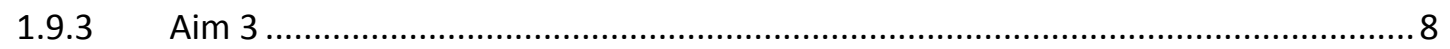

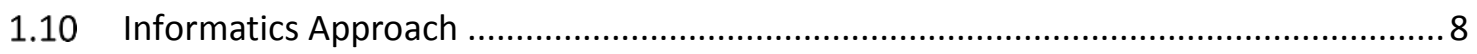

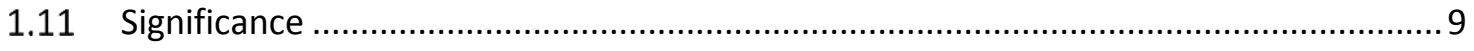

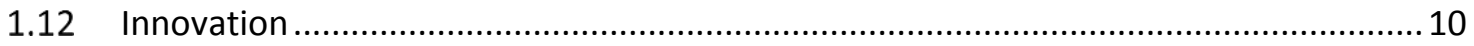

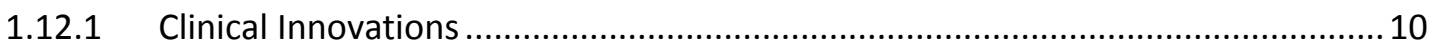

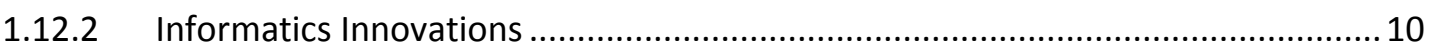

Chapter 2 : Patient-Related Risk Factors: A Systematic Review ............................................. 12

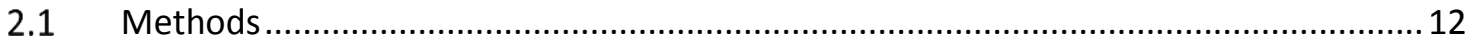

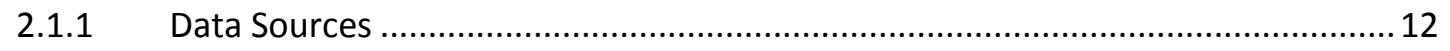

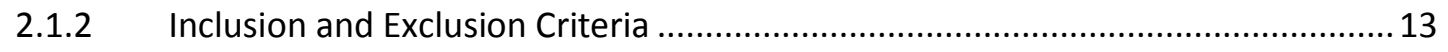

2.1.3 Study Selection and Data Extraction ............................................................... 13 


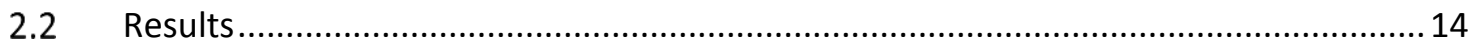

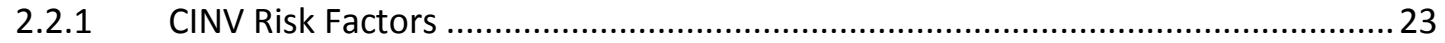

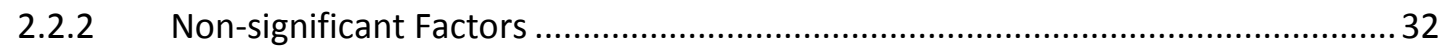

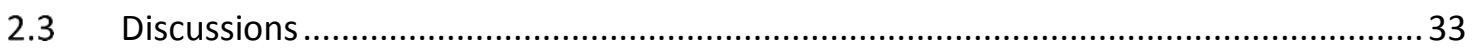

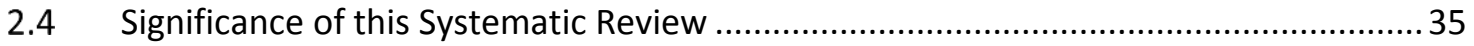

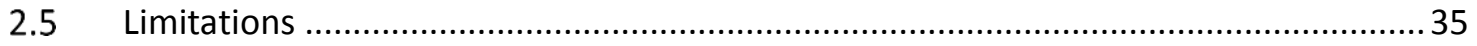

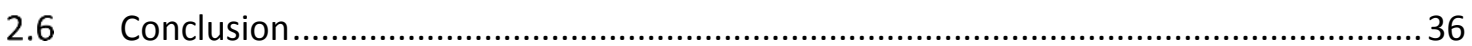

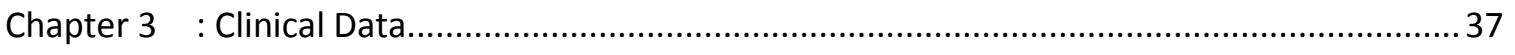

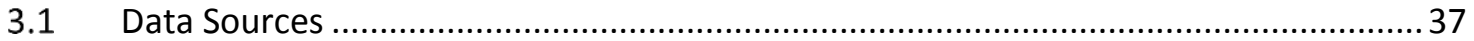

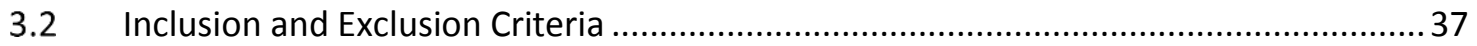

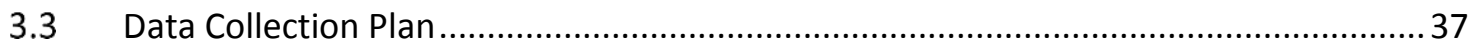

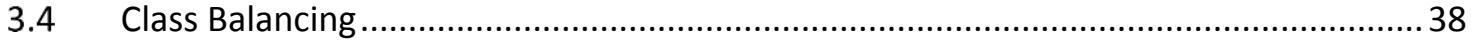

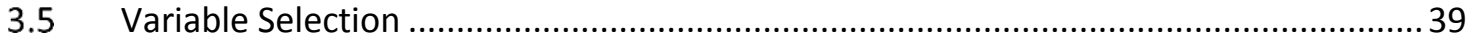

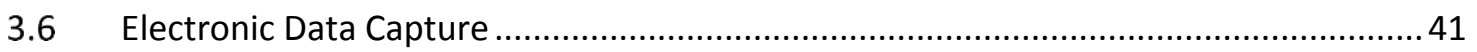

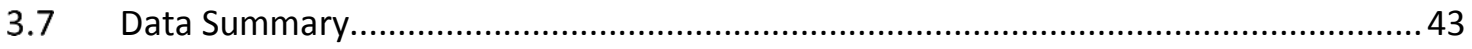

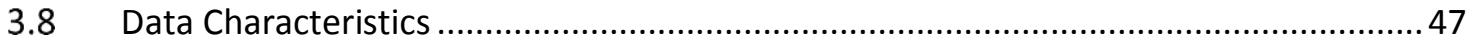

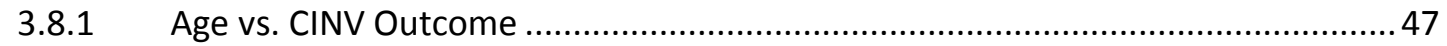

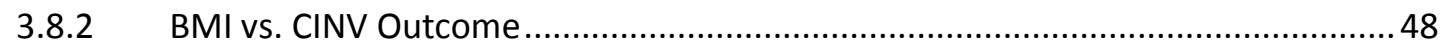

3.8.3 Number of Comorbidities vs. CINV Outcome …................................................... 49

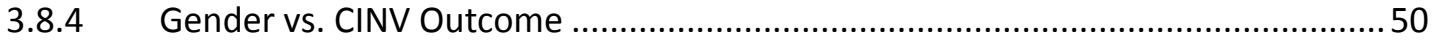

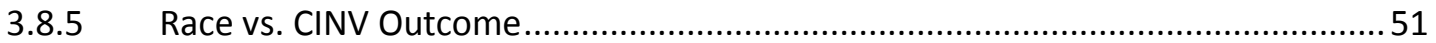

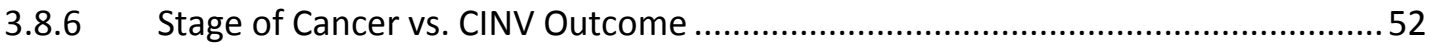

3.8.7 Alcohol Consumption vs. CINV Outcome ............................................................... 53

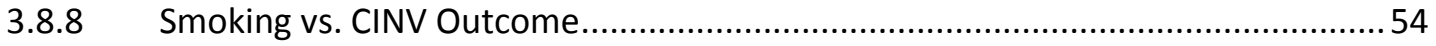

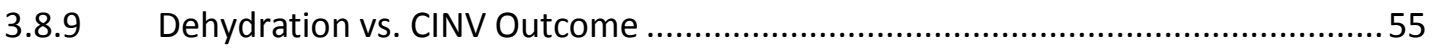

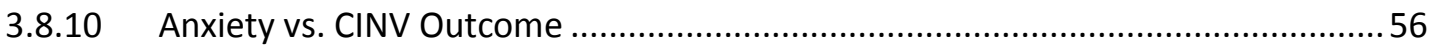

3.8.11 Number of Previous Chemotherapy vs. CINV Outcome …....................................57

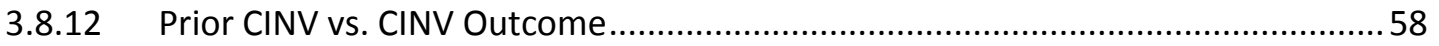

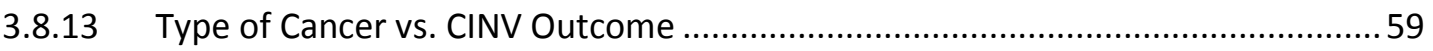

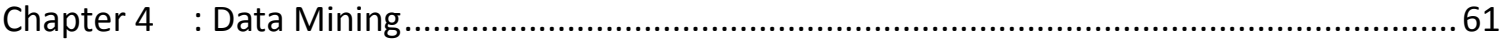

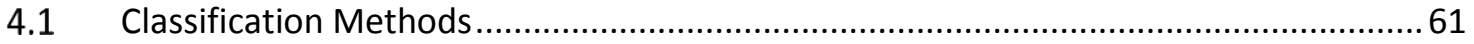

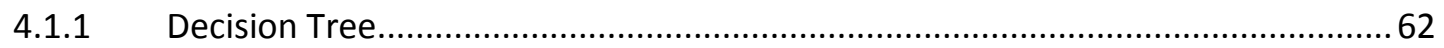




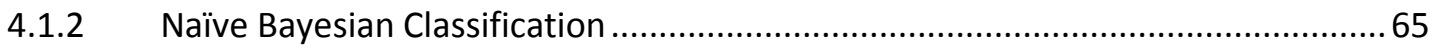

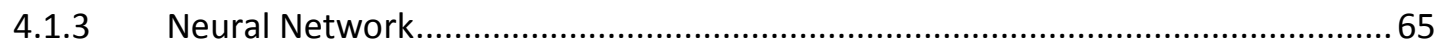

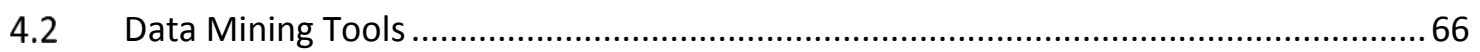

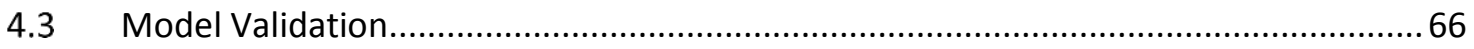

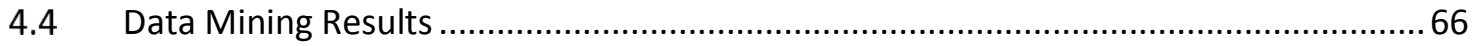

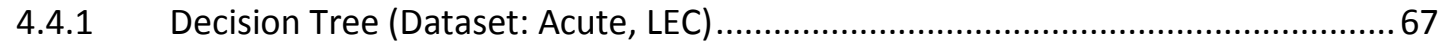

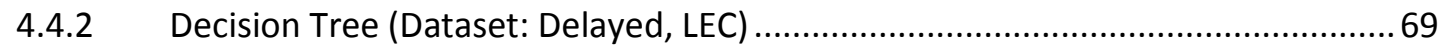

4.4.3 Decision Tree (Dataset: Acute, MEC) ................................................................. 71

4.4.4 Decision Tree (Dataset: Delayed, MEC) …......................................................... 73

4.4.5 Decision Tree (Dataset: Acute, HEC) ……........................................................ 75

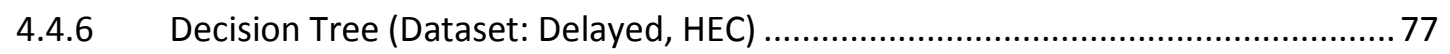

Chapter 5 : Mining Context Sensitive Associative Classification Rules....................................... 80

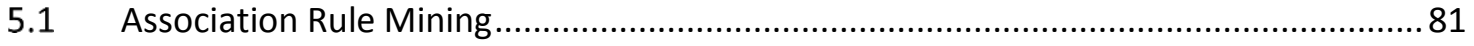

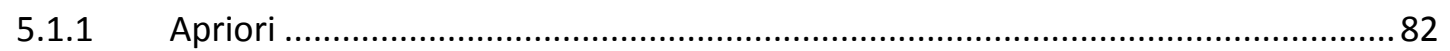

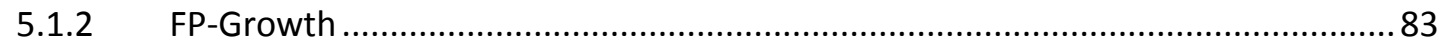

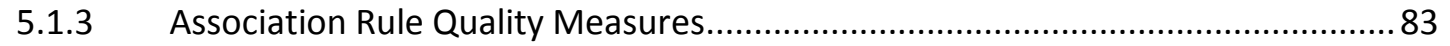

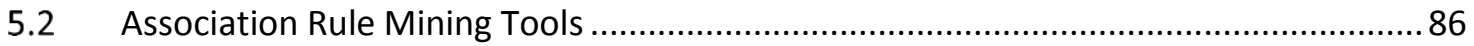

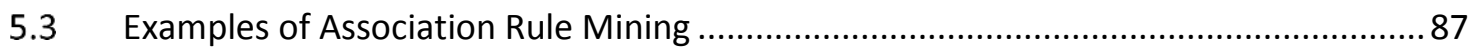

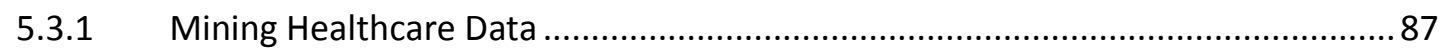

5.3.2 Mining Association of PubMed Search Tags ....................................................... 88

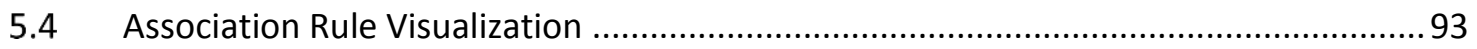

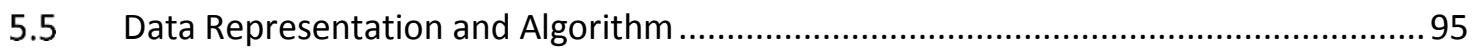

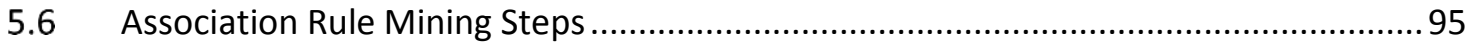

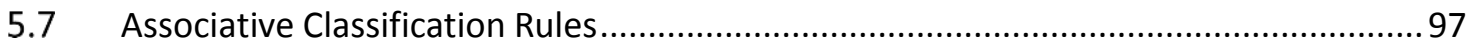

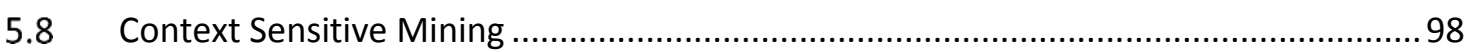

5.9 Mining Context Sensitive Associative Classification Rules Using Regular Algorithms. 102

5.10 Context Sensitive Frequent Associative Classification Tree (CS-FACT) ........................ 104

5.11 Mining Context Sensitive Associative Classification Rule using CS-FACT ....................112

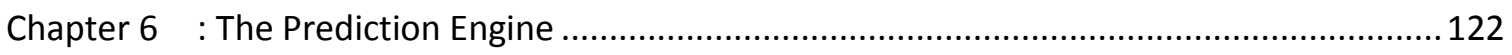

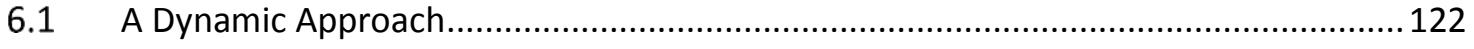

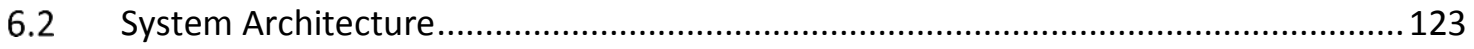

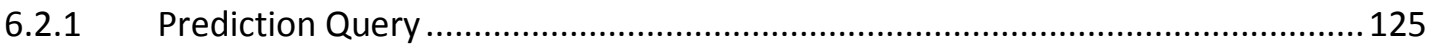




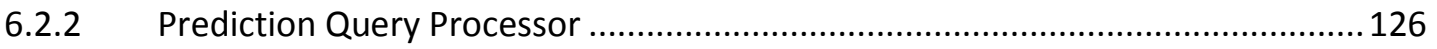

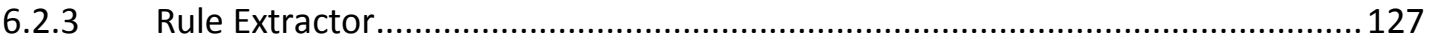

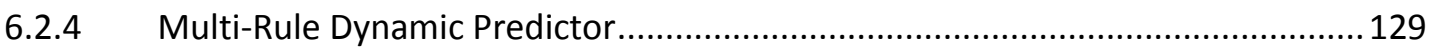

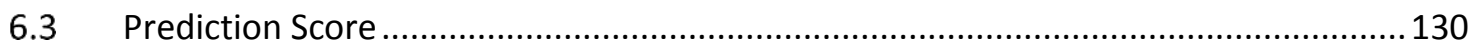

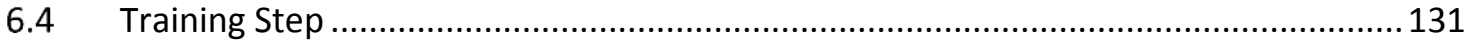

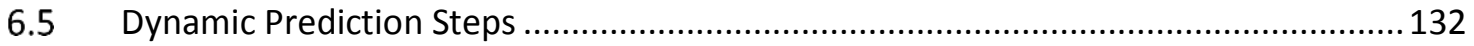

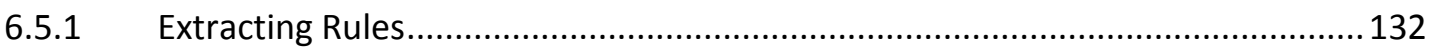

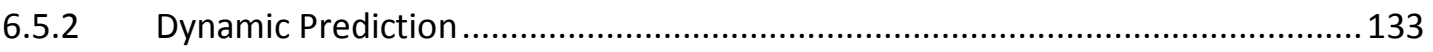

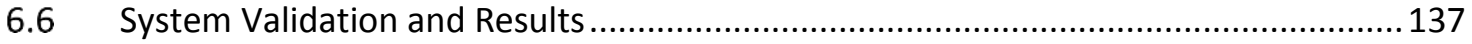

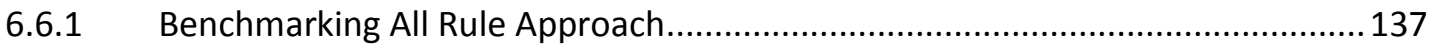

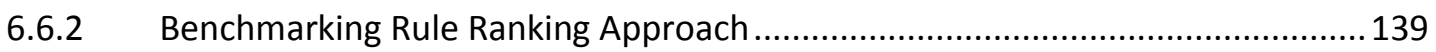

6.6.3 Prediction Using Any Number of Variables......................................................... 140

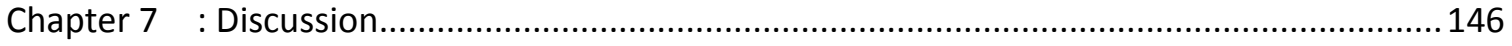

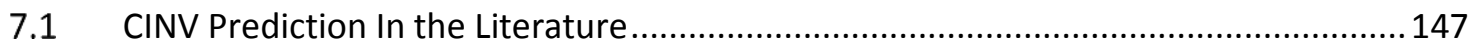

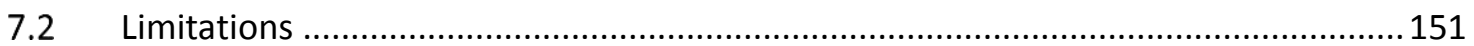

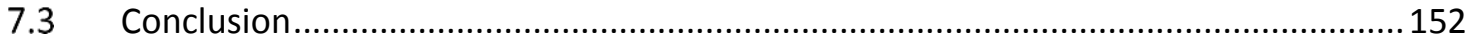

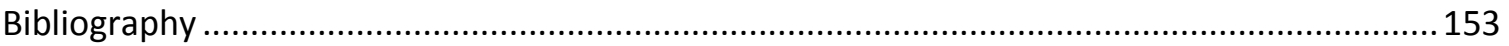

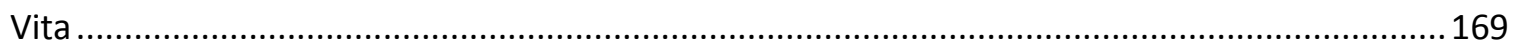




\section{LIST OF TABLES}

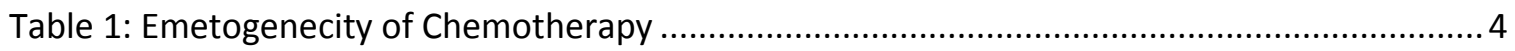

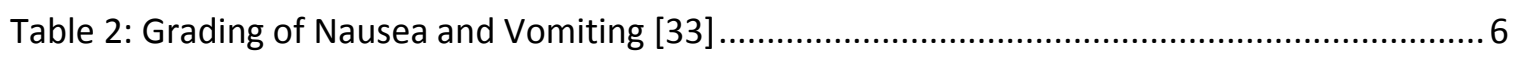

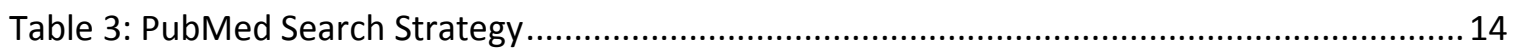

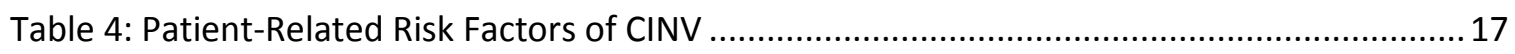

Table 5: Patient-Related Factors as the Significant Risk Factors of CINV ................................... 22

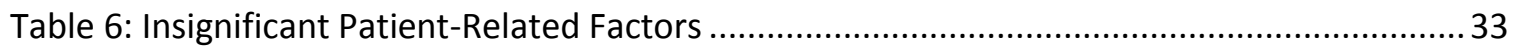

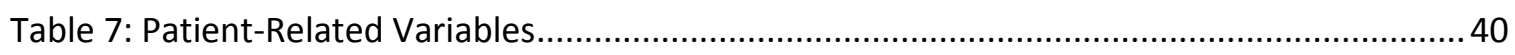

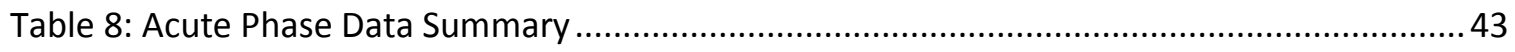

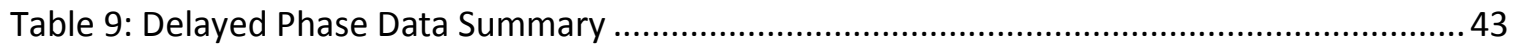

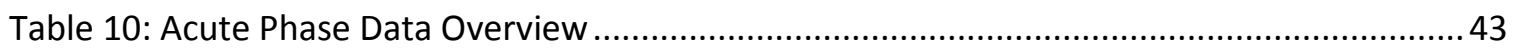

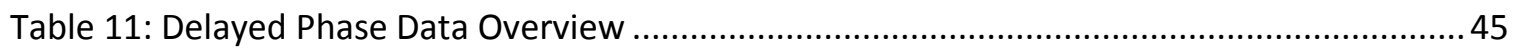

Table 12: Example Transactions for Illustrating Support, Confidence and Lift.............................84

Table 13: Example of Transactions for Illustrating Independence (Lift=1) ............................... 86

Table 14. Association Rules Consisting of Informational Tags only ........................................... 90

Table 15. Association Rules Consisting of Navigational Tags Only .............................................92

Table 16: Examples of Context Sensitive Associative Classification Rules from a Class Imbalanced

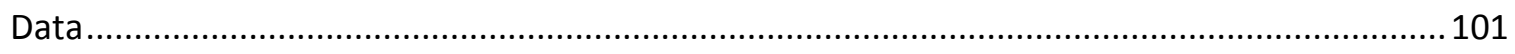

Table 17: Performance of Risk Scoring Algorithm for Acute CINV ........................................... 148

Table 18: Performance of Risk Scoring Algorithm for Delayed CINV ....................................... 149

Table 19: Performance of three CINV logistic regression models by Molassiotis et al. (2013) [70] 


\section{LIST OF FIGURES}

Figure 1: Overall 5-Year Relative Survival Rate of Cancer [9] ................................................ 2

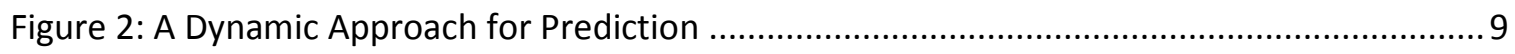

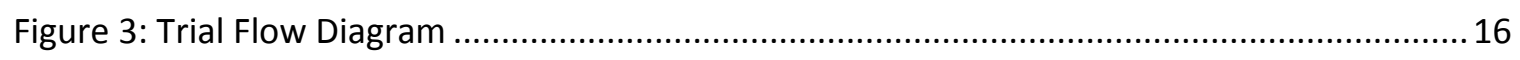

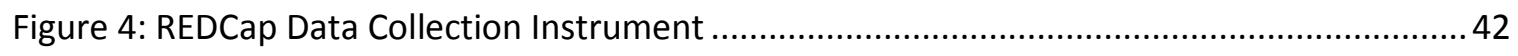

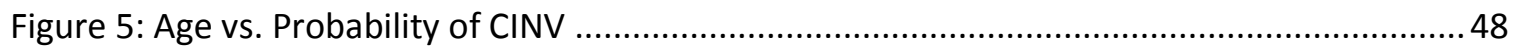

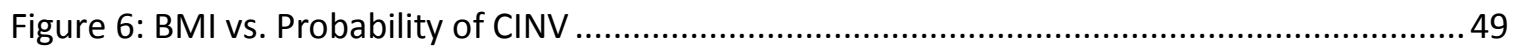

Figure 7: Number of Comorbidities vs. Probability of CINV ................................................. 50

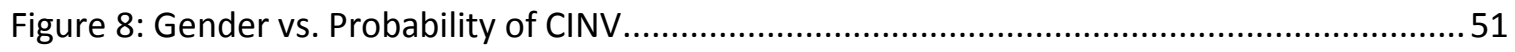

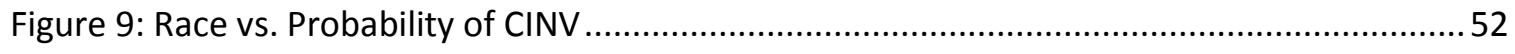

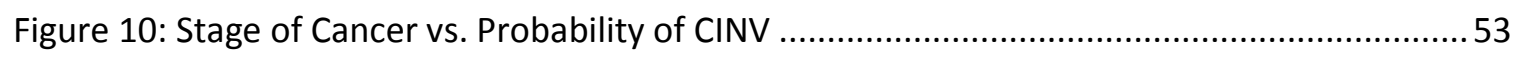

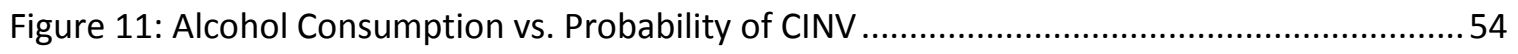

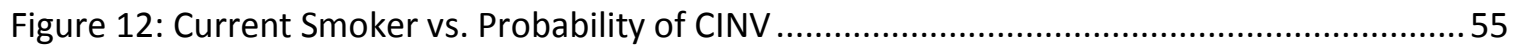

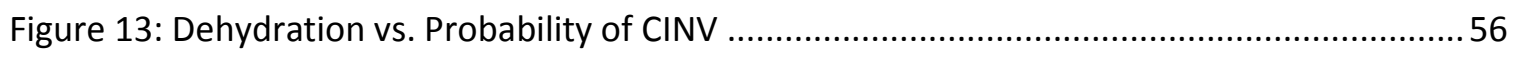

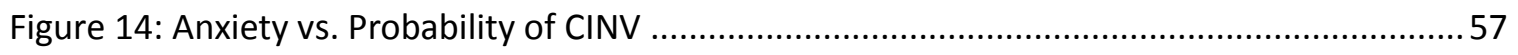

Figure 15: Number of Previous Chemotherapy vs. Probability of CINV ....................................58

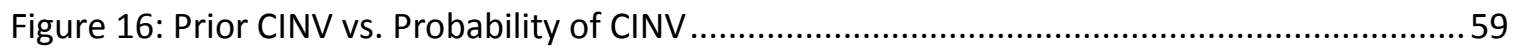

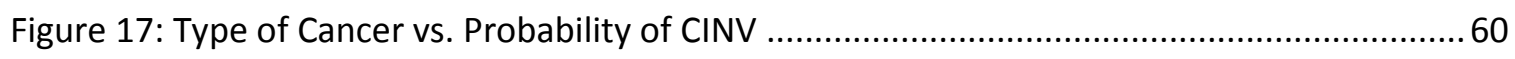

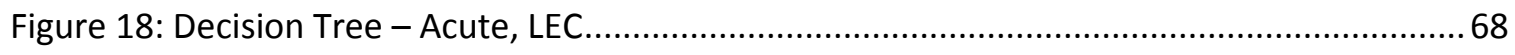

Figure 19: Prediction Result on Acute Phase and Low Emetogenic Chemotherapy Dataset .........69

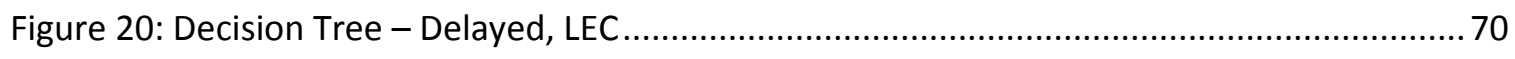

Figure 21: Prediction Result on Delayed Phase and Low Emetogenic Chemotherapy Dataset .... 71

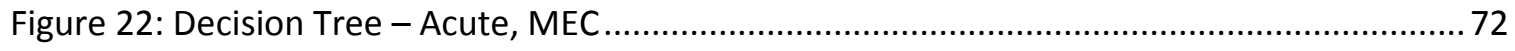


Figure 23: Prediction Result on Acute Phase and Moderate Emetogenic Chemotherapy Dataset

Figure 24: Decision Tree - Delayed, MEC

Figure 25: Prediction Result on Delayed Phase and Moderate Emetogenic Chemotherapy

Dataset 75

Figure 26: Decision Tree - Acute, HEC. 76

Figure 27: Prediction Result on Acute Phase and High Emetogenic Chemotherapy Dataset ....... 77

Figure 28: Decision Tree - Delayed, HEC 78

Figure 29: Prediction Result on Delayed Phase and High Emetogenic Chemotherapy Dataset.... 79

Figure 30: Association Rule Example 82

Figure 31. Visualization of association rules consisting of informational tags only 94

Figure 32. Visualization of association rules consisting of navigational/bibliographic tags only .. 94

Figure 33: Association Rule Mining Steps 96

Figure 34: Number of Association Rule Growth Chart .96

Figure 35: Processing Time for Generating Association Rules 97

Figure 36: Associative Classification Rule 98

Figure 37: Example of Contexts from the CINV Dataset 99

Figure 38: Context Sensitive Associative Classification Rule 100

Figure 39: Steps for Mining Context Sensitive Associative Classification Rule using Regular Algorithms 103

Figure 40: Number of Context Sensitive Associative Classification Rules 104

Figure 41: Example of Transactions for Generating Context Sensitive Associative Classification Tree - (a) Transaction, (b) Sorted item list, (c) Sorted Transactions 107

Figure 42: Construction of CS-FACT - (a) Insert Transaction \#1 and (b) Insert Transaction \#2 ... 108 
Figure 43: Construction of CS-FACT - Insert Transaction \#3 ................................................ 108

Figure 44: Construction of CS-FACT - Insert Transaction \#4 ..................................................... 109

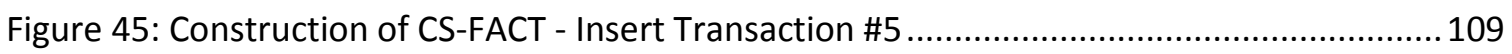

Figure 46: Construction of CS-FACT - Insert Transaction \#6 ................................................ 110

Figure 47: Construction of CS-FACT - Insert Transaction \#7 ................................................... 110

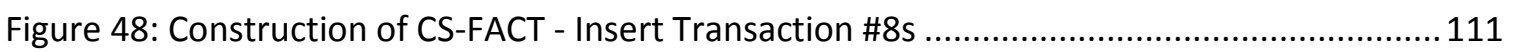

Figure 49: Construction of CS-FACT - Insert Transaction \#9 ................................................ 111

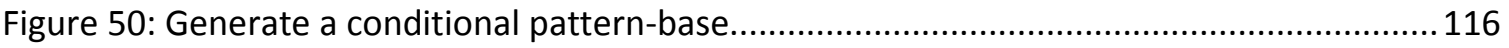

Figure 51: Conditional Frequent Pattern Tree for a Class ..................................................... 117

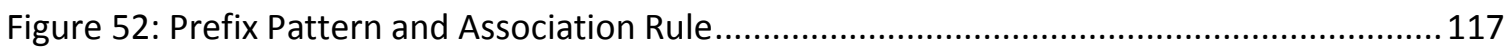

Figure 53: Conditional Frequent Pattern Tree for a Prefix Pattern ..........................................118

Figure 54: Context Sensitive Associative Classification Rules.................................................118

Figure 55: Mining Support Count for Antecedent ........................................................... 120

Figure 56: Steps for Mining Context Sensitive Associative Classification Rule using CS-FACT ....120

Figure 57: Processing Time for Generating Context Sensitive Associative Classification Rules

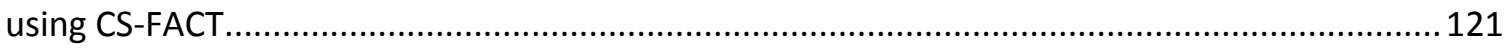

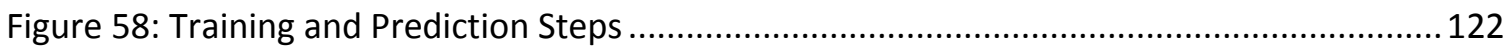

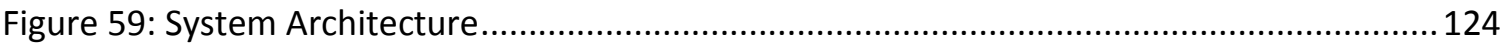

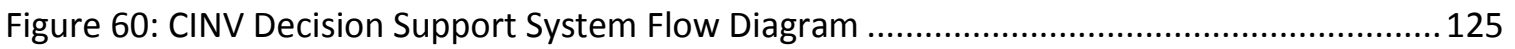

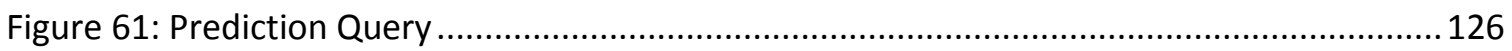

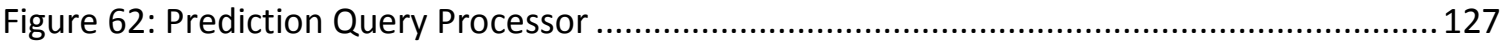

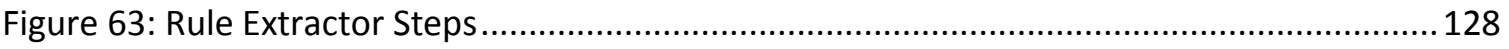

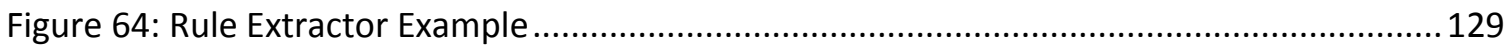

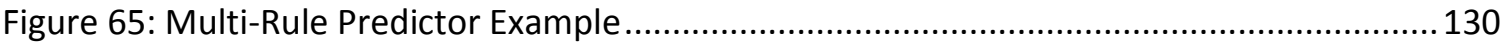


Figure 66: Reduction of Number of Rules on an Imbalanced Dataset

Figure 67: Multi-Rule Dynamic Predictor -All Rule Approach .................................................. 133

Figure 68: Multi-Rule Dynamic Predictor -Rule Ranking Approach ........................................ 135

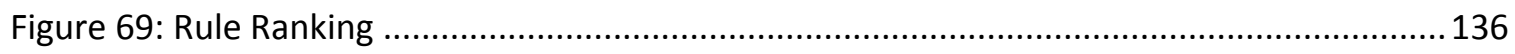

Figure 70: Benchmarking - All Rule Approach................................................................ 138

Figure 71: Comparison of Prediction Engine with Other Algorithms ..................................... 140

Figure 72: Loss of prediction capacity - Unavailable or Unreported Variable Situation............. 141

Figure 73: Comparison of Prediction with Incomplete Query (dehydration is missing in the query)

Figure 74: Comparison of Prediction with Incomplete Query (anxiety is missing in the query) .143

Figure 75: Comparison of Prediction with Incomplete Query (prior_cinv is missing in the query)

Figure 76: Comparison of Prediction with Incomplete Query (dehydration \& anxiety is missing in the query). 144

Figure 77: Comparison of Prediction with Incomplete Query (dehydration \& prior_cinv is missing in the query). 144

Figure 78: Comparison of Prediction with Incomplete Query (anxiety \& prior_cinv is missing in the query). 145

Figure 79: Comparison of Prediction with Incomplete Query (dehydration, anxiety \& prior_cinv is missing in the query) 145

Figure 80: CINV Prediction from the Literature vs. the Prediction Engine... .151 


\section{LIST OF EQUATIONS}

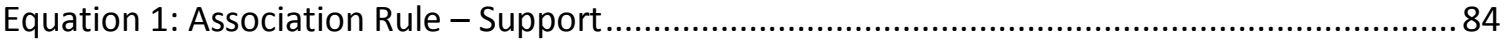

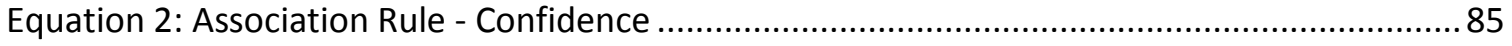

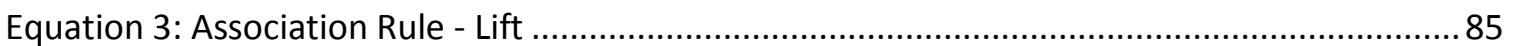

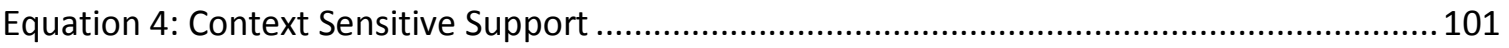

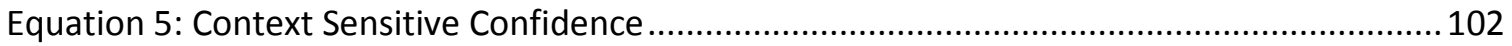

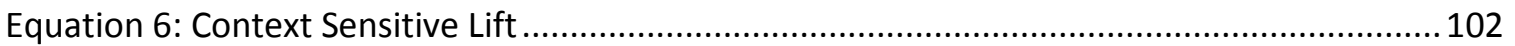

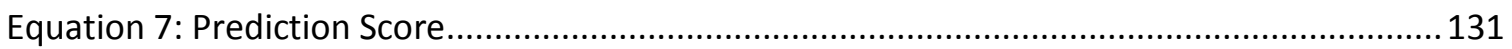




\section{LIST OF ALGORITHMS}

Algorithm 1: Construction of Context Sensitive Frequent Associative Classification Tree .........106

Algorithm 2: Mining Context Sensitive Associative Classification Rule using CS-FACT ...............115

Algorithm 3: Mining Support Count for an Itemset using a CS-FACT Tree ..................................119

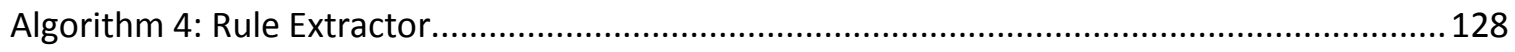




\section{ORGANIZATION OF THE DISSERTATION}

Chapter 1 provides an introduction to the clinical problem of chemotherapy induced nausea and vomiting (CINV). Chapter 2 provides a systematic review on the patient-related risk factors of CINV. Chapter 3 presents the data collection strategy, data overview and characteristics of the data. Chapter 4 presents the results of data mining using various traditional algorithms. There are a total of six datasets (i.e., contexts) and all the datasets were analyzed individually. Chapter 5 presents a new technique for mining context sensitive associative classification rules. Chapter 6 present a novel and dynamic prediction engine that has the capacity to build a highly precise prediction system while preserving the context-sensitiveness in one single system. The prediction performance of the system outperformed many popular prediction methods and all the results of CINV risk prediction published in the literature. Chapter 7 discusses the improvements that was made by this project and concludes the dissertation. 


\section{ABSTRACT}

Cancer remains the second major cause of death in the United States over the last decade. Chemotherapy is a core component of nearly every cancer treatment plan. Chemotherapy-Induced Nausea and Vomiting (CINV) are the two most dreadful and unpleasant side-effects of chemotherapy for cancer patients. The consequences of CINV include: (1) impaired life quality, (2) poor social life, (3) burden on economy due to loss of workdays, (4) increased healthcare cost, and (5) denial of chemotherapy due to unendurable CINV. There are three clinical guidelines (ASCO, NCCN, and MASCC/ESMO) for the management of CINV. Several patient-specific factors affect the risk of CINV. However, none of the guidelines consider those factors. Not all of patients have the similar emetic risk of CINV. Despite the improvements in CINV management, as many as two-thirds of chemotherapy patients still experience some degree of CINV. As a result, physicians use their personal experiences for CINV treatment, which leads to inconsistent managements of CINV. The overall objective of this study is to improve the prevention of CINV using precise, personalized and evidence-based antiemetic treatment before chemotherapy. Physicians receive feedback about CINV risks of patients from a CINV decision support system based on patient-specific factors. This objective was achieved by accomplishing clinical innovations through the discovery of combined relationships of various patient-specific factors for causing CINV, and informatics innovations through the development of a novel, precise and dynamic prediction engine for practicing precision and personalized medicine in CINV prevention. The approach presented in this dissertation can be applied to any other clinical predictions. 


\section{CHAPTER 1 : CHEMOTHERAPY INDUCED NAUSEA AND VOMITING (CINV)}

\subsection{CANCER}

Early diagnosis of cancer and the advancement in its treatments [1-6] have greatly increased the overall five-year relative and absolute survival rates (Figure 1) [5,7-11], but cancer remains the second major cause of mortality after cardiovascular mortality in the United States over the last decade [1,10-12]. The percentage of total mortality for the cardiovascular diseases is only $0.9 \%$ higher than that of the cancer in 2010 [12]. The US National Center for Health Statistics estimated that a total of 19 million adults in the US have ever been diagnosed with cancer [13]. Today, more cancer patients are treated than ever before with increasingly complex regimens. In cancer, tumors are formed due to fast, uncontrolled, and limitless growth of cells that can invade adjacent tissues and/or travel to distant sites (metastases) $[14,15]$. The overall goal of cancer treatments is to remove or destroy those cancerous cells. The main treatment plans for cancer include surgery, radiation therapy, and chemotherapy [16-18]. The goal of surgery is to remove the tumor and some tissues around it. Radiotherapy and/or chemotherapy is used to kill the cancerous 
cells. High-frequency rays are used in radiotherapy, and cytotoxic antineoplastic drugs are used in chemotherapy. The drugs used in chemotherapy are also known as chemotherapeutic agents.

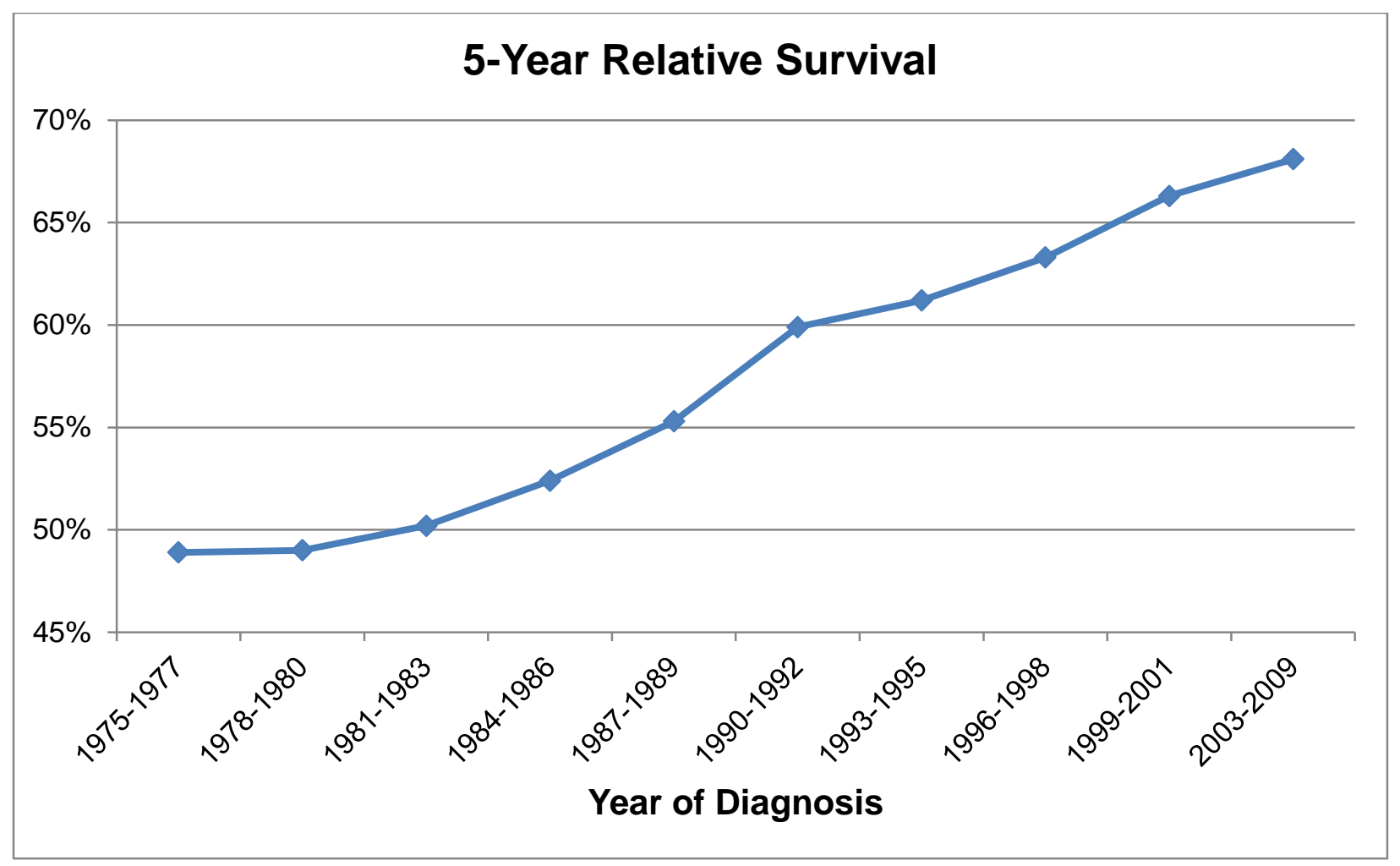

Figure 1: Overall 5-Year Relative Survival Rate of Cancer [9]

\subsection{CHEMOTHERAPY FOR CANCER TREATMENT}

Chemotherapy is a core component of nearly every cancer treatment plan [6]. A large number of cancer patients receive some type of chemotherapy. Hawkins and Grunberg (2009) [17] reported that as many as 1 million Americans receive chemotherapy each year. In cancer, cells are fast-growing, and form a tumor. A chemotherapeutic agent works by entering into the bloodstream and damaging every fast-growing cell as it divides [19]. In addition to destroying tumor cells, a chemotherapeutic agent also damages normal 
cells that are fast-growing, including the cells in skin [20,21], bone marrow [22], hair follicles $[23,24]$, and digestive tract $[25,26]$. As a result, chemotherapy causes many side effects such as nausea, vomiting, hair loss, appetite changes, anemia constipation, and diarrhea among others. [20-28].

\subsection{CHEMOTHERAPY INDUCED NAUSEA AND VOMITING}

Chemotherapy-induced nausea and vomiting (CINV) are the two most dreadful and unpleasant side-effects of chemotherapy [27-32]. The US National Cancer Institute (NCI) Common Terminology Criteria for Adverse Events (CTCAE) v4.0 defined nausea as "a disorder characterized by a queasy sensation and/or the urge to vomit" and vomiting as "a disorder characterized by the reflexive act of ejecting the contents of the stomach through the mouth" [33]. The pathophysiology of CINV is a complex process. There are two neurotransmitters involved in vomiting in two different pathways: (1) the serotonin is involved in the peripheral pathway, and (2) a neuropeptide named "substance P" is involved in the central pathway [34,35]. First, the peripheral pathway is associated with the acute phase of CINV that occurs within the first 24 hours of chemotherapy administration. This pathway is primarily located in the gastrointestinal tract, where chemotherapy causes the release of serotonin. CINV occurs when serotonin binds to the 5hydroxytryptamine $\left(5-\mathrm{HT}_{3}\right)$ receptors and sends a signal to the vomiting center in the medulla $[34,35]$. Second, the central pathway is associated with the delayed phase of CINV that occurs after the first 24 hours of chemotherapy administration and may persist up to one week. This pathway is located in the brain, where chemotherapy triggers the release of substance $\mathrm{P}$ that binds to the $\mathrm{NK}_{1}$ receptor in the vomiting center causing CINV $[34,35]$. 


\subsection{GUIDELINES FOR CINV MANAGEMENT}

There are several antiemetic guidelines for the management of CINV such as the American Society of Clinical Oncology (ASCO) guideline [36,37], the National Comprehensive Cancer Network $(\mathrm{NCCN})$ guideline [38], and the guideline from the Multinational Association of Supportive Care in Cancer (MASCC) in cooperation with the European Society of Medical Oncology (ESMO) [39]. The guideline-recommended standard antiemetic prophylaxis for the management of CINV considers only the emetogenecity of the chemotherapeutic agents. The emetogenecity of chemotherapy is divided into four emetic risk categories: (1) minimal, (2) low, (3) moderate, and (4) high (Table 1). The management of CINV is a complex process since it has two different phases involving different pathophysiology. Furthermore, several patient-related risk factors affect the risk of CINV but none of the guidelines considers those factors [40]. As seen in the emetic risk categories (Table 1), not all patients have the similar emetic risk of CINV. Despite the improvements in CINV management, as many as two-thirds of the patients still experience some degree of CINV; several recent studies reported various percentages of CINV cases with use of antiemetics: 28\% [41], 38-52\% [42], 56.1\% [43], 61.2\% [44], and $62 \%$ [45]. As a result, physicians use their personal experiences with the treatment of CINV, which leads to inconsistent management of CINV [46].

\section{Table 1: Emetogenecity of Chemotherapy}

\begin{tabular}{|c|c|}
\hline Degree of Emetogenecity & $\begin{array}{c}\text { \% of Patients Who Suffer from } \\
\text { CINV Without Antiemetics }\end{array}$ \\
\hline Minimal & $<10 \%$ \\
\hline Low (LEC) & $10 \%-30 \%$ \\
\hline Moderate (MEC) & $30 \%-90 \%$ \\
\hline High (HEC) & $>90 \%$ \\
\hline
\end{tabular}




\subsection{CONSEQUENCES OF CINV}

There are many consequences of CINV that affect both patients and the healthcare system as a whole. First, CINV impairs the quality of life in cancer patients due to nutritional deficits, dehydration, and electrolyte imbalance. [44,45,47-49]. Second, CINV makes it difficult to socialize with family and friends leading to a poor social life $[44,50]$. Third, CINV creates an economic burden due to loss of workdays [44,51-53]. Fourth, CINV increases healthcare costs due to CINV-related outpatient visits, hospitalization, and cost of drugs. [44,49,51-56]. Fifth, cancer patients may discontinue chemotherapy due to intolerable CINV leading to poor treatment outcomes, treatment failure, or even death [29,57-59]. In addition to the fact that none of the CINV guidelines considers patientrelated risk factors, these problems are the motivation of this study.

\subsection{CINV GRADES}

The NCI defined three grades for nausea and five grades for vomiting (Table 2). With any chemotherapy, grade 1 (mild) CINV are minimally expected and not considered as clinically significant. However, grade 2 (moderate) to grade 3 (severe) CINV are clinically significant and needs antiemetic prophylaxis (prevention) in-advance before chemotherapy. If CINV is not prevented appropriately, the patient may end having life threatening event (grade 4) leading to death (grade 5). 
Table 2: Grading of Nausea and Vomiting [33]

\begin{tabular}{|c|c|c|}
\hline & Nausea & Vomiting \\
\hline Definition & $\begin{array}{l}\text { A disorder characterized by a queasy } \\
\text { sensation and/or the urge to vomit. }\end{array}$ & $\begin{array}{l}\text { A disorder characterized by the } \\
\text { reflexive act of ejecting the contents of } \\
\text { the stomach through the mouth. }\end{array}$ \\
\hline \multicolumn{3}{|l|}{ Grades } \\
\hline $\begin{array}{l}\text { Grade } 1 \\
\text { (Mild) }\end{array}$ & $\begin{array}{l}\text { Loss of appetite without alteration in } \\
\text { eating habits }\end{array}$ & $\begin{array}{l}1 \text { - } 2 \text { episodes (separated by } 5 \text { minutes) } \\
\text { in } 24 \mathrm{hrs}\end{array}$ \\
\hline $\begin{array}{l}\text { Grade } 2 \\
\text { (Moderate) }\end{array}$ & $\begin{array}{l}\text { Oral intake decreased without } \\
\text { significant weight loss, dehydration or } \\
\text { malnutrition }\end{array}$ & $\begin{array}{l}3-5 \text { episodes (separated by } 5 \text { minutes) } \\
\text { in } 24 \text { hrs }\end{array}$ \\
\hline $\begin{array}{l}\text { Grade } 3 \\
\text { (Severe) }\end{array}$ & $\begin{array}{l}\text { Inadequate oral caloric or fluid intake; } \\
\text { tube feeding, TPN, or hospitalization } \\
\text { indicated }\end{array}$ & $\begin{array}{l}\text { >=6 episodes (separated by } 5 \text { minutes) } \\
\text { in } 24 \mathrm{hrs;} \text { tube feeding, TPN or } \\
\text { hospitalization indicated }\end{array}$ \\
\hline $\begin{array}{l}\text { Grade } 4 \\
\text { (Life Threatening) }\end{array}$ & N/A & $\begin{array}{l}\text { Life-threatening consequences; urgent } \\
\text { intervention indicated }\end{array}$ \\
\hline $\begin{array}{l}\text { Grade } 5 \\
\text { (Death) }\end{array}$ & N/A & Death \\
\hline
\end{tabular}

\subsection{CARE OF CINV}

If a patient started to have CINV, stopping the consequences become very hard. Thus, using antiemetic treatments before chemotherapy for the prevention of CINV is imperative. The prescription for antiemetics should consider the guideline recommendations as well as the associative effect of various patient-specific risk factors.

\subsection{PROBLEM STATEMENT}

Despite the improvements in CINV management, as many as two-thirds of the patients still experience some degree of CINV. Several patient-specific factors affect the risk of CINV but none of the guidelines (NCCN, ASCO, MASCC, and ESMO) considers those factors. As a result, physicians cannot fully rely on the guidelines and they use their own experiences using CINV. Consequently, the management of CINV is inconsistent 
among physicians since their decisions are subjective to their experiences with the CINV management.

\subsection{OVERALL OBJECTIVE, SCOPE AND AIMS}

The overall objective of this project is to improve the prevention of CINV using precise, personalized and evidence-based antiemetic treatment before chemotherapy. Physicians receive feedback about CINV risks of patients from a CINV decision support system based on patient-specific factors. The data mining techniques are used to develop a novel and dynamic prediction engine that provides a CINV treatment decision based on the patient-specific factors. This objective will be achieved by accomplishing three specific aims covering data collection, clinical innovations in the discovery of combined relationships of various patient-specific factors for causing CINV, and informatics innovations in the development of a novel dynamic prediction engine for practicing precision and personalized medicine at the point-of-care.

\subsubsection{Aim 1}

The first aim of the project was to collect potential CINV related risk-factor data by chart review from the MU Ellis Fischel Cancer Center. Two separate datasets for acute and delayed phase of CINV have been collected. Chapter 2 presents the results of a systematic review study on the patient-related risk factors. Chapter 3 presents the details on the data collection plan and the characteristics of data. 


\subsubsection{Aim 2}

The second aim of the project was to apply data mining techniques for predictive analytics. Predicting the risk of CINV before chemotherapy for a chemotherapy patient is important so that physician can make data-driven and evidence-based decision about CINV treatment recommendations. We applied various classification algorithms in this endeavor (Chapter 4).

\subsubsection{Aim 3}

The third aim of the project was to develop a data mining-based novel and dynamic prediction engine employing the patient-related variables. The prediction engine discovers how the patient-related variables and the emetogenecity of chemotherapy are associated with the risk of CINV (Chapter 5). Unlike pre-computed prediction models using training data, this dynamic prediction engine is revisable in real-time based on the actual treatment outcome (Chapter 6). In addition, this prediction engine provides a decision even though an important variable is unknown at the point-of-care.

\subsection{INFORMATICS APPROACH}

The final goal is to develop a data mining-based novel and dynamic prediction engine that can precisely perform the prediction job for determining the patients who are at higher risk of causing CINV based on the patients' personal risk factors. The approach involves data mining for generating a classification model and use the model for prediction. We have developed a novel prediction technique using associative classification rules. Using this technique, the system receives a prediction query, retrieves a set of supported 
associative classification rules in real-time and dynamically generates a context sensitive prediction result. Figure 2 presents the overall project design.

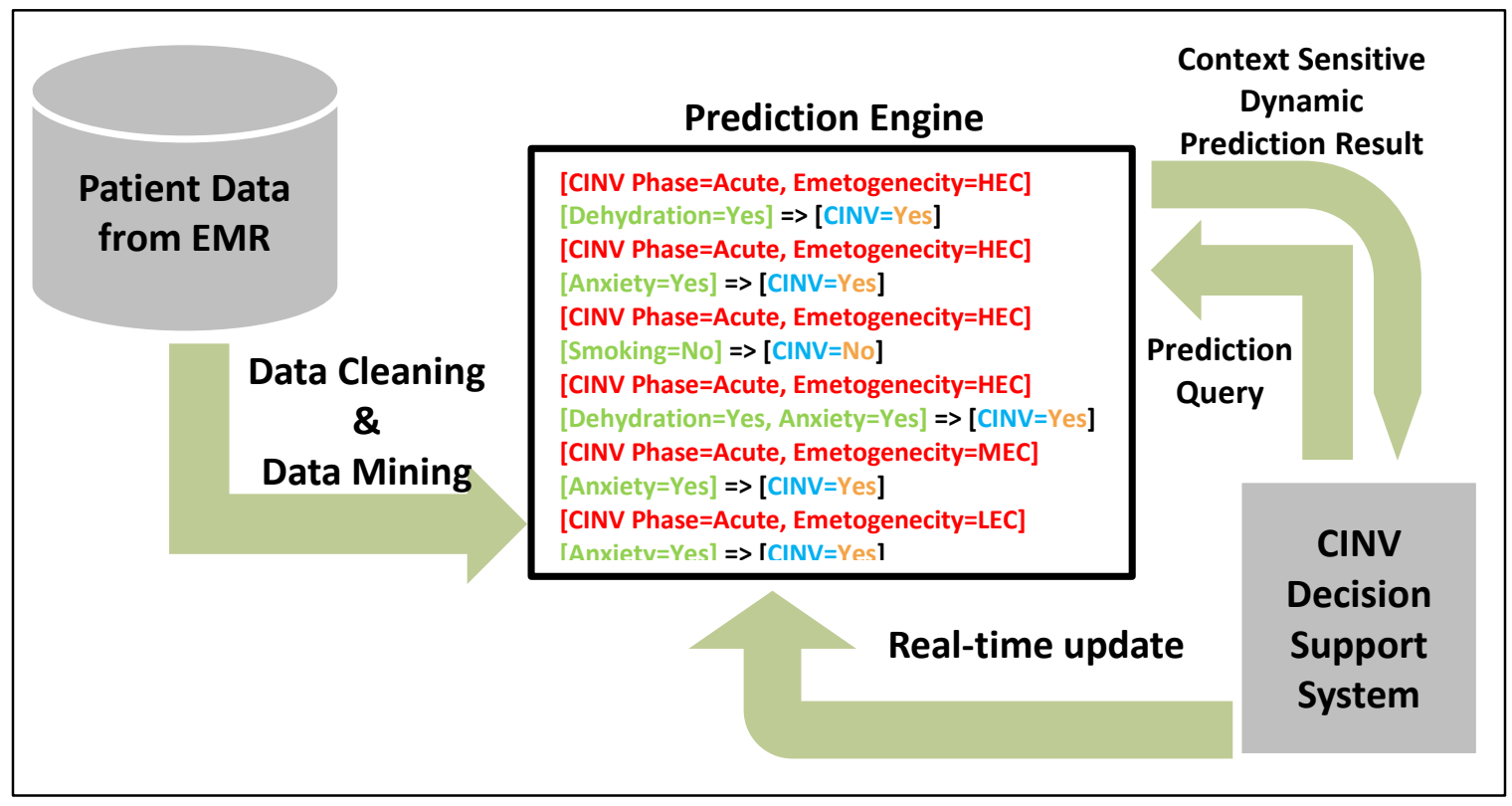

Figure 2: A Dynamic Approach for Prediction

\subsection{SIGNIFICANCE}

As described in section 1.5, CINV has significant negative consequences in personal, social, clinical and economic matters. As a result, a good control of this fearful side-effect is very important for minimizing these consequences. CINV guidelines are based on the classification of the chemotherapy drugs into four vague emetic risk groups. Physicians cannot anticipate the exact probability of CINV for a certain patient using this classification scheme. The guidelines do not consider the patient-related risk factors [40]. In fact, the current NCCN guideline (Version 2.2015) for antiemetic therapy stated that "patient factors" and "prior experience with antiemetics" should be considered in addition to the emetic risk of chemotherapy [60]. 
The main treatment is using antiemetics before chemotherapy for preventing CINV. Over treating all patients is not a viable solution for many reasons: (1) every patients are not at higher risk of CINV, (2) antiemetic medications have their own side-effects, and (3) antiemetic medications are costly. If physicians know which patients are at higher risk of CINV, they can make a precise treatment plan for individual patients. This project solves this important clinical problem by developing a dynamic prediction engine.

\subsection{INNOVATION}

This project contributes innovation in two categories: (1) clinical innovations, (2) informatics innovations.

\subsubsection{Clinical Innovations}

To date, the combined relationships of various patient-related factors for causing CINV was unknown. This project discovers these relationships, improves clinical outcomes through data mining and helps physicians for practicing personalized and precision medicine for the management of CINV. The contribution of this project significantly improves overall cancer care and reduces overall cost of care.

\subsubsection{Informatics Innovations}

The prediction engine proposed in this project is new in comparing to the traditional data mining approaches. The prediction engine consists of several novel features. First, this method does not suffer from the issue of losing prediction capacity in a situation when an important variable is unavailable or unknown at the point-of-care. This can still make a

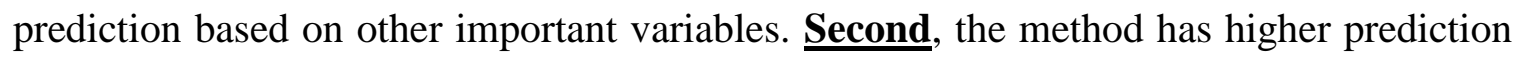


power since multiple important rules are used for a prediction query. A new prediction scoring algorithm has been developed for combining multiple rules; this algorithm generates a prediction score for each class of interest that helps with the prediction process. Third, the prediction engine keeps all unimportant (rare) rules. Fourth, unlike precomputed prediction models using training data such as decision trees, the prediction engine is dynamic (revisable in real-time). This dynamic process may benefit the unimportant rules to become important as more data is added in real-time. Fifth, the approach proposed in this project can be adapted for any other clinical predictions. $\underline{\text { Sixth}}$, the engine can grow from ground-zero by learning in real-time and start providing predictions after minimal growth. 


\section{CHAPTER 2 : PATIENT-RELATED RISK FACTORS: A SYSTEMATIC REVIEW}

Studies have reported that patient-related factors considerably impact the risk of Chemotherapy-Induced Nausea and Vomiting (CINV). In this chapter, a systematic review of the literature is presented that identifies and summarizes patient-related factors (scattered over various articles) that significantly impact a patient's risk of CINV. Oncologists can use these factors to identify patients at risk for CINV prior to the initiation of a chemotherapy regimen, so that they can focus on more aggressive treatment options for those high risk patients for better treatment outcomes and avoid further complications. The reporting of this systematic review follows the "Preferred Reporting Items for Systematic Reviews and Meta-Analyses (PRISMA)” guideline [61].

\subsection{METHODS}

\subsubsection{Data Sources}

The MEDLINE citation database was searched in March, 2015 for eligible articles using PubMed. The search terms are related to the following terms: antiemetics, chemotherapy-induced nausea and vomiting (CINV) and risk factors. We used a comprehensive search strategy not to miss relevant documents in MEDLINE. Table 3 presents the entire search strategy. 


\subsubsection{Inclusion and Exclusion Criteria}

The inclusion criteria include: (1) studies that reported patient-related risk factors of CINV, (2) any kind of clinical studies such as prospective, retrospective, clinical trial, cross-sectional, cross-over, and case-control studies etc., (3) studies that do not provide any concomitant cancer treatment such as radiotherapy or surgery, and (4) the patient population is adult. We excluded any articles that were not written in English.

\subsubsection{Study Selection and Data Extraction}

The study selection was performed in two steps. In the first step, we read the titles and abstracts of the citations by the search query in order to screen the articles based on the inclusion/exclusion criteria. In the second step, we read the full-text of the citations selected by the first step. The search criteria did not include any limitation on publication date; the earliest eligible article was published in 1989.

We abstracted information from the eligible full-text articles. Table 4 presents the extracted information that includes the study type, country name, number of patients, type of cancer, emetic risk of the chemotherapy, potential patient-related factors that are included in the data analysis and the factors that were identified as the predictors of CINV. 
Table 3: PubMed Search Strategy

\begin{tabular}{|c|c|}
\hline Search Topic & Search Strategy \\
\hline Antiemetics & $\begin{array}{ll}\text { 1. } & \text { "antiemetics"[All Fields] } \\
\text { 2. } & \text { "antiemetics"[MeSH Terms] } \\
\text { 3. } & \text { "antiemetics"[Pharmacological Action] } \\
\text { 4. } & \text { OR }(1,2,3)\end{array}$ \\
\hline CINV & 5. "CINV"[All Fields] \\
\hline Chemotherapy & $\begin{array}{l}\text { 6. "drug therapy"[MeSH Terms] } \\
\text { 7. "drug"[All Fields] AND "therapy"[All Fields] } \\
\text { 8. "drug therapy"[All Fields] } \\
\text { 9. "chemotherapy"[All Fields] } \\
\text { 10. "antineoplastic agents"[MeSH Terms] } \\
\text { 11. "antineoplastic"[All Fields] AND "agents"[All Fields] } \\
\text { 12. "antineoplastic agents"[All Fields] } \\
\text { 13. "antineoplastic"[All Fields] AND "agent"[All Fields] } \\
\text { 14. "antineoplastic agent"[All Fields] } \\
\text { 15. "antineoplastic agents"[Pharmacological Action] } \\
\text { 16. OR (6-15) }\end{array}$ \\
\hline Nausea & $\begin{array}{l}\text { 17. "nausea"[MeSH Terms] } \\
\text { 18. "nausea"[All Fields] } \\
\text { 19. OR }(17,18)\end{array}$ \\
\hline Vomiting & $\begin{array}{l}\text { 20. "vomiting"[MeSH Terms] } \\
\text { 21. "vomiting"[All Fields] } \\
\text { 22. OR }(20,21)\end{array}$ \\
\hline $\begin{array}{l}\text { Chemotherapy Induced Nausea } \\
\text { and/or Vomiting (CINV) }\end{array}$ & $\begin{array}{l}\text { 23. } \text { OR }(19,22) \\
\text { 24. } \operatorname{AND}(16,23) \\
\text { 25. } \text { OR }(5,24)\end{array}$ \\
\hline Risk Factors & $\begin{array}{l}\text { 26. "risk factors"[MeSH Terms] } \\
\text { 27. "risk"[All Fields] AND ("factors"[All Fields] OR "factor"[All } \\
\text { Fields]) } \\
\text { 28. "risk factors"[All Fields] } \\
\text { 29. "risk factor"[All Fields] } \\
\text { 30. OR (26-29) }\end{array}$ \\
\hline English Language & 31. English[Lang] \\
\hline Final Search Query & 32. $\operatorname{AND}(4,25,30,31)$ \\
\hline
\end{tabular}

\subsection{RESULTS}

Figure 3 presents the trial flow diagram of identifying the eligible articles for this study. A total of 390 articles were identified through the searches conducted in PubMed. We identified additional 11 articles by reviewing the list of references that were not retrieved by the query. The titles and abstracts of those 401 articles were then screened for eligibility. A total of 337 articles were excluded because these articles do not qualify the inclusion criteria described in the methods section. The remaining 64 articles were assessed 
in full-text for eligibility and data extraction. We excluded a total of 34 articles after fulltext review: (1) 20 articles were excluded because risk factors are cited as the background information of the articles, (2) 3 articles discussed the pharmacogenomics of CINV, (3) 5 articles considered concomitant radiotherapy/surgery, (4) 3 articles presented the risk factors associated with issues other than CINV, (5) 2 articles reported the evaluation of previously developed risk prediction models, and (6) 1 article performed the study on pediatric patients. Finally, we included 30 articles in this study discovering the patientrelated risk factors $(n=30)$.

Table 4 presents the patient-related risk factors from the 30 studies. Among those 30 studies, 13 studies were multicenter study and 17 studies were single-center study. The study types include retrospective $(n=2)$, prospective $(n=17)$, clinical trial $(n=8)$, crosssectional $(n=1)$, cross-over $(n=1)$, and case-control $(n=1)$. The studies were performed in various countries around the world: USA $(n=6)$, Canada $(n=4)$, Singapore $(n=3)$, Italy $(n=2)$, Germany $(n=1)$, Japan $(n=5)$, Malaysia $(n=1)$, Netherlands $(n=1)$, Sweden $(n=1)$, Taiwan $(n=1)$, UK $(n=1)$; four studies were performed in multiple countries $(n=4)$. A total of 14,302 patient-records were analyzed by those 30 studies. Nine studies focused on any cancer including both solid and hematological malignancies $(n=9)$, nine studies focused on the solid cancers only $(n=9)$, and twelve studies focused on a specific type of cancer: breast cancer $(n=7)$, colorectal cancer $(n=1)$, gastrointestinal cancer $(n=1)$, lung cancer $(n=1)$, gynecologic cancer $(n=1)$ and ovarian cancer $(n=1)$. Most of the research studied high and moderate emetic risk of the chemotherapies: (1) 9 studies were on the high emetic risk only, (2) 8 studies were on the moderate emetic risk only, (3) 11 studies were on both high 
and moderate risk chemotherapies, and (4) two studies were on high, moderate and low risk chemotherapies.

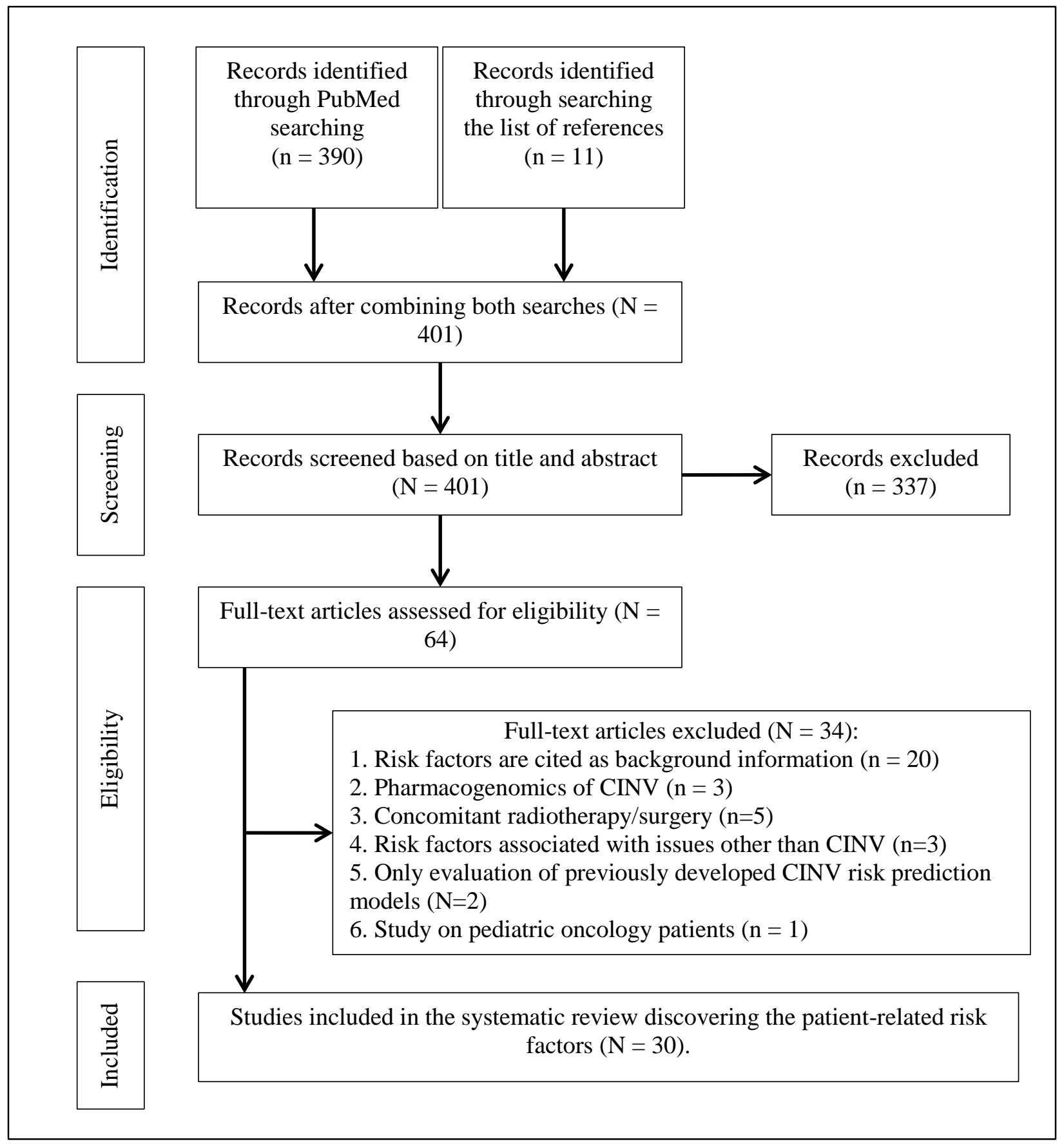

Figure 3: Trial Flow Diagram 
Table 4: Patient-Related Risk Factors of CINV

\begin{tabular}{|c|c|c|c|c|c|c|c|}
\hline \begin{tabular}{|l|} 
Author \\
(Year)
\end{tabular} & Study Type & Country & $\begin{array}{c}\# \\
\text { Patients } \\
\end{array}$ & Cancer Types & $\begin{array}{c}\text { Emetic } \\
\text { Risk }\end{array}$ & Variables Used in the Study & Identified Risk Factors \\
\hline \begin{tabular}{|l} 
Kitazaki et \\
al. $(2015)$ \\
{$[62]$}
\end{tabular} & Prospective & Japan & 133 & Lung & $\begin{array}{l}\text { High and } \\
\text { moderate }\end{array}$ & $\begin{array}{l}\text { Age, sex, history of CINV, history of motion } \\
\text { sickness, alcohol intake }\end{array}$ & None \\
\hline $\begin{array}{l}\text { Tamura et al. } \\
(2015)[63]\end{array}$ & $\begin{array}{l}\text { Multicenter, } \\
\text { prospective, } \\
\text { observational }\end{array}$ & Japan & 1910 & Any Cancer & $\begin{array}{l}\text { High and } \\
\text { moderate }\end{array}$ & $\begin{array}{l}\text { Sex, history of motion sickness, history of } \\
\text { pregnancy-related nausea/vomiting, history of } \\
\text { morning sickness, alcohol intake, age }\end{array}$ & $\begin{array}{l}\text { Young age, female gender, } \\
\text { history of motion sickness, } \\
\text { history of pregnancy-related } \\
\text { nausea/vomiting, history of } \\
\text { morning sickness and \# of } \\
\text { drinks per week }<5\end{array}$ \\
\hline $\begin{array}{l}\text { Molassiotis } \\
\text { et al. }(2014) \\
{[64]}\end{array}$ & $\begin{array}{l}\text { Multicenter, } \\
\text { prospective, } \\
\text { observational }\end{array}$ & $\begin{array}{l}\text { France, } \\
\text { Italy, Spain, } \\
\text { Belgium, } \\
\text { Sweden, } \\
\text { UK, Austria, } \\
\text { and The } \\
\text { Netherlands } \\
\end{array}$ & 991 & Solid & $\begin{array}{l}\text { High and } \\
\text { moderate }\end{array}$ & $\begin{array}{l}\text { Age, sex, cancer type, history of CINV, anxiety, } \\
\text { nausea before chemotherapy, Expectancy of CINV }\end{array}$ & $\begin{array}{l}\text { Age }<50, \text { nausea before } \\
\text { chemotherapy, history of } \\
\text { CINV, anxiety, female gender }\end{array}$ \\
\hline \begin{tabular}{|l|} 
Murakami et \\
al. $(2014)$ \\
{$[65]$}
\end{tabular} & $\begin{array}{l}\text { Prospective, } \\
\text { observational }\end{array}$ & Japan & 92 & Any cancer & Moderate & Age, sex, history of previous chemo & Age $<67$, female gender \\
\hline \begin{tabular}{|l|} 
Furukawa et \\
al. $(2014)$ \\
{$[66]$}
\end{tabular} & Prospective & Japan & 72 & $\begin{array}{l}\text { Gynecologic } \\
\text { cancer }\end{array}$ & Moderate & $\begin{array}{l}\text { Age, BMI, comorbidity (hypertension), type of } \\
\text { cancer, menopause, alcohol intake, smoking } \\
\text { history, history of motion sickness, } \\
\text { defecations/bowel movement, history of } \\
\text { pregnancy-related nausea/vomiting, history of } \\
\text { chemo, history of radiotherapy, ascites }\end{array}$ & $\begin{array}{l}\text { Comorbidity (hypertension), } \\
\text { history of pregnancy-related } \\
\text { nausea/vomiting }\end{array}$ \\
\hline $\begin{array}{l}\text { Celio et al. } \\
(2013)[67]\end{array}$ & $\begin{array}{l}\text { Multicenter, } \\
\text { Retrospective }\end{array}$ & $\begin{array}{l}\text { Austria, } \\
\text { Germany, } \\
\text { Italy and } \\
\text { Spain } \\
{[68,69]}\end{array}$ & 405 & Breast & Moderate & Age & None \\
\hline
\end{tabular}




\begin{tabular}{|c|c|c|c|c|c|c|c|}
\hline \begin{tabular}{|l|} 
Author \\
(Year)
\end{tabular} & Study Type & Country & \begin{tabular}{c|}
$\#$ \\
Patients \\
\end{tabular} & Cancer Types & $\begin{array}{c}\text { Emetic } \\
\text { Risk }\end{array}$ & Variables Used in the Study & Identified Risk Factors \\
\hline $\begin{array}{l}\text { Molassiotis } \\
\text { et al. (2013) } \\
{[70]}\end{array}$ & $\begin{array}{l}\text { Multicenter, } \\
\text { prospective, } \\
\text { observational }\end{array}$ & UK & 336 & Solid & $\begin{array}{l}\text { High, } \\
\text { moderate } \\
\text { and low }\end{array}$ & $\begin{array}{l}\text { Age, expectation of CINV, state anxiety, trait } \\
\text { anxiety, symptom distress score, sex, stage of } \\
\text { cancer, cancer type, chemotherapy cycle no., } \\
\text { alcohol, history of nausea/vomiting (including } \\
\text { motion sickness, pregnancy-related or vestibular } \\
\text { dysfunction), comorbidities, and amount of sleep } \\
\text { prior to treatment in hours }\end{array}$ & $\begin{array}{l}\text { Young age, history of } \\
\text { nausea/vomiting, trait anxiety, } \\
\text { pain, first cycle of } \\
\text { chemotherapy }\end{array}$ \\
\hline $\begin{array}{l}\text { Sekine et al. } \\
(2013)[71]\end{array}$ & Prospective & Japan & 1,549 & Any cancer & $\begin{array}{l}\text { High and } \\
\text { moderate }\end{array}$ & Sex, age, performance status, smoking, alcohol & $\begin{array}{l}\text { Female gender, age }<55 \\
\text { years, poor performance } \\
\text { status, low alcohol intake } \\
\text { (non-habitual drinker) and } \\
\text { non-smoking habit }\end{array}$ \\
\hline $\begin{array}{l}\text { Chan et al. } \\
(2012) \text { [72] }\end{array}$ & Prospective & Singapore & 156 & Gastrointestinal & Moderate & $\begin{array}{l}\text { Age, sex, race, alcohol, anxiety, number of prior } \\
\text { chemo, prior CINV, history of motion sickness and } \\
\text { morning sickness }\end{array}$ & $\begin{array}{l}\text { Prior CINV and moderate to } \\
\text { severe anxiety }\end{array}$ \\
\hline \begin{tabular}{|l|} 
Fleishman et \\
al. $(2012)$ \\
{$[73]$}
\end{tabular} & $\begin{array}{l}\text { Cross- } \\
\text { sectional }\end{array}$ & USA & 41 & Colorectal & Moderate & $\begin{array}{l}\text { Age, Sex, Type of cancer, Stage of cancer, history } \\
\text { of motion sickness, history of morning sickness, } \\
\text { history of chemotherapy, prior CINV }\end{array}$ & $\begin{array}{l}\text { Female gender and prior } \\
\text { CINV }\end{array}$ \\
\hline $\begin{array}{l}\text { Yap et al. } \\
(2012)[74]\end{array}$ & $\begin{array}{l}\text { Prospective, } \\
\text { observational }\end{array}$ & Singapore & 710 & Solid & $\begin{array}{l}\text { High and } \\
\text { moderate }\end{array}$ & 21 symptoms related to anxiety & $\begin{array}{l}\text { Fear of dying, fear of the } \\
\text { worst, unable to relax, hot/cold } \\
\text { sweats, nervousness, faintness, } \\
\text { numbness }\end{array}$ \\
\hline $\begin{array}{l}\text { Higa et al. } \\
(2012) \text { [75] }\end{array}$ & Case-control & USA & 25 & Solid & Moderate & Substance P, 5-HIAA/creatinine & $\begin{array}{l}\text { Pretreatment ratio of } \\
\text { substance-p and 5- } \\
\text { HIAA/creatinine }>70\end{array}$ \\
\hline $\begin{array}{l}\text { Celio et al. } \\
(2012) \text { [76] }\end{array}$ & $\begin{array}{l}\text { Multicenter, } \\
\text { phase III } \\
\text { randomized } \\
\text { trial }\end{array}$ & Italy & 324 & Solid & Moderate & Age, sex, alcohol & Age $<50$ \\
\hline $\begin{array}{l}\text { Hilarius et } \\
\text { al. }(2012) \\
{[48]}\end{array}$ & $\begin{array}{l}\text { Multicenter, } \\
\text { prospective, } \\
\text { observational }\end{array}$ & Netherlands & 277 & Any cancer & $\begin{array}{l}\text { High and } \\
\text { moderate }\end{array}$ & $\begin{array}{l}\text { Age, sex, race, type of cancer, alcohol, pregnancy- } \\
\text { related nausea/vomiting, history of motion } \\
\text { sickness, performance status }\end{array}$ & $\begin{array}{l}\text { Age }<65, \text { female gender, \# of } \\
\text { drinks per week }<5\end{array}$ \\
\hline $\begin{array}{l}\text { Bourdeanu et } \\
\text { al. }(2012) \\
{[77]}\end{array}$ & Retrospective & USA & 358 & Breast & High & $\begin{array}{l}\text { Age, race, depression, diabetes, education, GERD, } \\
\text { private/public insurance, marital status, medical } \\
\text { history, religion, cancer stage, weight and alcohol }\end{array}$ & $\begin{array}{l}\text { Asian race, private insurance, } \\
\text { age } \leq 50 \text { and GERD }\end{array}$ \\
\hline
\end{tabular}

$\mapsto$ 


\begin{tabular}{|c|c|c|c|c|c|c|c|}
\hline \begin{tabular}{|l|} 
Author \\
(Year)
\end{tabular} & Study Type & Country & \begin{tabular}{c|}
$\#$ \\
Patients
\end{tabular} & Cancer Types & $\begin{array}{l}\text { Emetic } \\
\text { Risk }\end{array}$ & Variables Used in the Study & Identified Risk Factors \\
\hline $\begin{array}{l}\text { Warr et al. } \\
(2011)[78]\end{array}$ & $\begin{array}{l}\text { Multicenter, } \\
\text { randomized } \\
\text { phase III trial }\end{array}$ & $\begin{array}{l}\text { USA, } \\
\text { Germany, } \\
\text { Austria, } \\
\text { Canada, } \\
\text { Hong Kong, } \\
\text { Hungary, } \\
\text { Spain, UK, } \\
\text { Italy, } \\
\text { Australia, } \\
\text { and Greece } \\
\text { [79] }\end{array}$ & 866 & Breast & Moderate & $\begin{array}{l}\text { Age, alcohol, pregnancy-related nausea/vomiting, } \\
\text { history of morning and motion sickness, }\end{array}$ & $\begin{array}{l}\text { Age }<55, \# \text { of drinks per week } \\
<5, \text { history of morning } \\
\text { sickness }\end{array}$ \\
\hline $\begin{array}{l}\text { Hassan and } \\
\text { Yusoff } \\
(2010)[80]\end{array}$ & $\begin{array}{l}\text { Prospective, } \\
\text { observational }\end{array}$ & Malaysia & 158 & Breast & High & $\begin{array}{l}\text { Age, Sex, Race (Malay, Chinese and Asian } \\
\text { Indians) }\end{array}$ & Race \\
\hline $\begin{array}{l}\text { Hesketh et } \\
\text { al. }(2010) \\
{[81]}\end{array}$ & $\begin{array}{l}\text { Multicenter, } \\
\text { randomized } \\
\text { phase III trial }\end{array}$ & $\begin{array}{l}\text { Argentina, } \\
\text { Brazil, } \\
\text { Chile, } \\
\text { Colombia, } \\
\text { Guatemala, } \\
\text { Mexico, } \\
\text { Peru, and } \\
\text { Venezuela, } \\
\text { USA and } 14 \\
\text { other } \\
\text { countries } \\
{[82,83]} \\
\end{array}$ & 1,043 & Solid & High & $\begin{array}{l}\text { Age, sex, alcohol, BMI, history of motion and } \\
\text { morning sickness, history of previous } \\
\text { chemotherapy, prior CINV }\end{array}$ & $\begin{array}{l}\text { Female gender, age }<65 \text {, \# of } \\
\text { drinks per week }<5\end{array}$ \\
\hline $\begin{array}{l}\text { Roscoe et al. } \\
(2010)[84]\end{array}$ & $\begin{array}{l}\text { Multicenter, } \\
\text { clinical trial }\end{array}$ & USA & 1,696 & Any cancer & $\begin{array}{l}\text { High and } \\
\text { moderate }\end{array}$ & $\begin{array}{l}\text { Cancer type, expectancies for nausea, pregnancy- } \\
\text { related nausea/vomiting, history of motion } \\
\text { sickness }\end{array}$ & $\begin{array}{l}\text { Breast cancer, young age, } \\
\text { expectancies and perceived } \\
\text { susceptibility to nausea }\end{array}$ \\
\hline $\begin{array}{l}\text { Shih et al. } \\
(2009) \text { [85] }\end{array}$ & $\begin{array}{l}\text { Prospective, } \\
\text { observational }\end{array}$ & Singapore & 91 & Breast & High & $\begin{array}{l}\text { Age, race, education level, alcohol, history of } \\
\text { motion sickness, history of morning sickness, } \\
\text { anxiety, prior CINV, frequency of chemotherapy } \\
\text { cycle, number of risk factors, chemotherapy cycle } \\
\text { no. }\end{array}$ & $\begin{array}{l}\text { Anxiety, prior CINV, history } \\
\text { of motion sickness }\end{array}$ \\
\hline
\end{tabular}




\begin{tabular}{|c|c|c|c|c|c|c|c|}
\hline \begin{tabular}{|l} 
Author \\
(Year)
\end{tabular} & Study Type & Country & \begin{tabular}{c|}
$\#$ \\
Patients
\end{tabular} & Cancer Types & $\begin{array}{c}\text { Emetic } \\
\text { Risk }\end{array}$ & Variables Used in the Study & Identified Risk Factors \\
\hline $\begin{array}{l}\text { Dranitsaris et } \\
\text { al. }(2009) \\
{[86]}\end{array}$ & Prospective & Canada & 200 & Any cancer & $\begin{array}{l}\text { High and } \\
\text { moderate }\end{array}$ & $\begin{array}{l}\text { Age, cancer type, cancer stage, comorbidities, } \\
\text { recent surgery within past } 3 \text { months, history of } \\
\text { motion sickness, history of morning sickness, } \\
\text { alcohol, prior CINV, chemotherapy cycle no., meal } \\
\text { prior to chemotherapy, number of hours of sleep } \\
\text { the night before the chemotherapy, expectation of } \\
\text { CINV, anxiety, non-prescription drugs for emesis } \\
\text { control before chemotherapy, nausea/vomiting } \\
\text { before chemotherapy }\end{array}$ & $\begin{array}{l}\text { Age } \leq 40, \text { gynecologic or } \\
\text { genitourinary cancer, cancer } \\
\text { stage I/II, no existing } \\
\text { comorbidity, \# of drinks per } \\
\text { week }<7, \text { chemotherapy cycle } \\
\text { no. }<3 \text {, non-prescription drugs } \\
\text { for emesis control before } \\
\text { chemotherapy }\end{array}$ \\
\hline $\begin{array}{l}\text { Petrella et al. } \\
(2009) \text { [87] }\end{array}$ & Prospective & Canada & 200 & Any cancer & $\begin{array}{l}\text { High and } \\
\text { moderate }\end{array}$ & $\begin{array}{l}\text { Age, cancer type, cancer stage, comorbidities, } \\
\text { recent surgery within past } 3 \text { months, history of } \\
\text { motion sickness, history of morning sickness, } \\
\text { alcohol, prior CINV, chemotherapy cycle no., meal } \\
\text { prior to chemotherapy, number of hours of sleep } \\
\text { the night before the chemotherapy, expectation of } \\
\text { CINV, anxiety, non-prescription drugs for emesis } \\
\text { control before chemotherapy, nausea/vomiting } \\
\text { before chemotherapy }\end{array}$ & $\begin{array}{l}\text { Age } \leq 40, \text { prior CINV, } \\
\text { nausea/vomiting before } \\
\text { chemotherapy, history of } \\
\text { morning sickness, non- } \\
\text { prescription drugs for emesis } \\
\text { control before chemotherapy, } \\
\text { chemotherapy cycle no. }<3, \\
\text { lower number of hours slept } \\
\text { before the night of } \\
\text { chemotherapy }\end{array}$ \\
\hline $\begin{array}{l}\text { Colagiuri et } \\
\text { al. }(2008) \\
{[88]}\end{array}$ & $\begin{array}{l}\text { Multicenter, } \\
\text { clinical trial }\end{array}$ & USA & 691 & Any cancer & High & $\begin{array}{l}\text { Age, sex, susceptibility to motion sickness, } \\
\text { expectancy of CINV }\end{array}$ & Expectancy of CINV \\
\hline $\begin{array}{l}\text { Booth et al. } \\
(2007) \text { [89] }\end{array}$ & $\begin{array}{l}\text { Multicenter, } \\
\text { prospective }\end{array}$ & Canada & 143 & Breast & $\begin{array}{l}\text { Moderate } \\
\text { and low }\end{array}$ & $\begin{array}{l}\text { Age, sex, cancer stage, comorbidities, history of } \\
\text { motion sickness, history of morning sickness, } \\
\text { alcohol, meal prior to chemotherapy, number of } \\
\text { hours slept during the preceding night, expectancy } \\
\text { of CINV, anxiety, recent surgery }\end{array}$ & $\begin{array}{l}\text { Age }<40, \text { no existing } \\
\text { comorbidity, recent surgery, } \\
\text { expectancy of CINV, \# of } \\
\text { drinks per week }<7, \text { no food } \\
\text { before chemotherapy, history } \\
\text { of morning sickness }\end{array}$ \\
\hline $\begin{array}{l}\text { Roscoe et al. } \\
(2004)[90]\end{array}$ & $\begin{array}{l}\text { Multicenter, } \\
\text { clinical trial }\end{array}$ & USA & 201 & Breast & $\begin{array}{l}\text { High and } \\
\text { moderate }\end{array}$ & $\begin{array}{l}\text { Patient demographics, pregnancy-related } \\
\text { nausea/vomiting, history of motion sickness, } \\
\text { expectancy of CINV }\end{array}$ & Expectancy of CINV \\
\hline $\begin{array}{l}\text { Liaw et al. } \\
(2003)[91]\end{array}$ & $\begin{array}{l}\text { Cross-over } \\
\text { trial }\end{array}$ & Taiwan & 400 & Solid & High & $\begin{array}{l}\text { Age, sex, performance status, previous } \\
\text { noncisplatin chemotherapy, cancer type }\end{array}$ & Female gender \\
\hline
\end{tabular}

ธ 


\begin{tabular}{|c|c|c|c|c|c|c|c|}
\hline \begin{tabular}{|l|} 
Author \\
(Year)
\end{tabular} & Study Type & Country & \begin{tabular}{c|c|}
$\#$ \\
Patients
\end{tabular} & Cancer Types & $\begin{array}{c}\text { Emetic } \\
\text { Risk }\end{array}$ & Variables Used in the Study & Identified Risk Factors \\
\hline $\begin{array}{l}\text { Osoba et al. } \\
(1997)[92]\end{array}$ & Prospective & Canada & 832 & Any cancer & $\begin{array}{l}\text { High and } \\
\text { moderate }\end{array}$ & $\begin{array}{l}\text { Age, sex, history of motion sickness, alcohol, } \\
\text { performance status, social functioning, disease site, } \\
\text { cancer type, food intake, drowsiness, pre- } \\
\text { chemotherapy nausea }\end{array}$ & $\begin{array}{l}\text { Social functioning }<70 \text {, pre- } \\
\text { chemotherapy nausea, female } \\
\text { gender, \# of drinks per week }< \\
10\end{array}$ \\
\hline $\begin{array}{l}\text { Hursti et al. } \\
(1996)[93]\end{array}$ & Clinical trial & Sweden & 101 & Ovarian & High & $\begin{array}{l}\text { Age, history of nausea/vomiting (including motion } \\
\text { sickness, pregnancy-related, or alcohol-related), } \\
\text { neuroticism, autonomic perception, and tumor } \\
\text { burden }\end{array}$ & Tumor burden $\geq 2 \mathrm{~cm}$, age $\geq 55$ \\
\hline $\begin{array}{l}\text { du Bois et al. } \\
(1992)[94]\end{array}$ & $\begin{array}{l}\text { Multicenter, } \\
\text { Prospective }\end{array}$ & Germany & 92 & Solid & High & $\begin{array}{l}\text { Age, sex, cancer type, number of previous } \\
\text { chemotherapy, previous antiemetics, cancer } \\
\text { recurrence (new/recurrence) }\end{array}$ & $\begin{array}{l}\text { Female gender, recurrent } \\
\text { cancer }\end{array}$ \\
\hline $\begin{array}{l}\text { Pollera et al. } \\
(1989) \text { [95] }\end{array}$ & Clinical trial & Italy & 209 & Solid & High & Age, sex, performance status, cancer type & $\begin{array}{l}\text { Age } \leq 55, \text { female gender and } \\
\text { poor performance status }\end{array}$ \\
\hline
\end{tabular}


Table 5: Patient-Related Factors as the Significant Risk Factors of CINV

\begin{tabular}{|c|c|c|}
\hline Variable Name & $\begin{array}{l}\text { \# of Studies Including the } \\
\text { Variable }\end{array}$ & $\begin{array}{c}\text { \# of Studies Finding the Variable as a } \\
\text { Significant Risk Factor }\end{array}$ \\
\hline Age & 26 & 16 \\
\hline Sex & 18 & 11 \\
\hline Alcohol Intake & 17 & 8 \\
\hline $\begin{array}{l}\text { History of CINV or } \\
\text { pregnancy-related } \\
\text { Nausea/Vomiting }\end{array}$ & 17 & 8 \\
\hline Anxiety & 8 & 5 \\
\hline Expectancy of CINV & 7 & 3 \\
\hline Chemotherapy Cycle No. & 4 & 3 \\
\hline Comorbidities & 6 & 4 \\
\hline Cancer Type & 12 & 2 \\
\hline $\begin{array}{l}\text { History of Morning } \\
\text { Sickness }\end{array}$ & 10 & 3 \\
\hline Performance Status & 5 & 2 \\
\hline Race & 5 & 2 \\
\hline $\begin{array}{l}\text { Use of Non-prescription } \\
\text { Drugs }\end{array}$ & 2 & 2 \\
\hline History of Motion Sickness & 17 & 2 \\
\hline Cancer Stage & 6 & 1 \\
\hline $\begin{array}{l}\text { Sleeping before } \\
\text { Chemotherapy }\end{array}$ & 4 & 1 \\
\hline Meal before chemotherapy & 4 & 1 \\
\hline Recent surgery & 3 & 1 \\
\hline Smoking History & 2 & 1 \\
\hline $\begin{array}{l}\text { Neurotransmitter } \\
\text { Measurements (Substance- } \\
\text { P, 5-HIAA/creatinine) }\end{array}$ & 1 & 1 \\
\hline Private/Public Insurance & 1 & 1 \\
\hline Social Functioning & 1 & 1 \\
\hline $\begin{array}{l}\text { Nausea/Vomiting before } \\
\text { Chemotherapy }\end{array}$ & 3 & 2 \\
\hline Tumor Burden & 1 & 1 \\
\hline $\begin{array}{l}\text { Cancer Recurrence } \\
\text { (new/recurrent) }\end{array}$ & 1 & 1 \\
\hline Symptom Distress Score & 1 & 1 \\
\hline
\end{tabular}


We present the patient-related factors in two categories: the factors that were identified as the risk factors of CINV and the factors that were included in the data analysis and found to be insignificant. In the following, the risk factors are discussed followed by the insignificant factors.

\subsubsection{CINV Risk Factors}

Table 5 presents a total of 26 patient-related factors that were found to be the significant risk factors of CINV in one or more studies. These 26 risk factors are: age, sex, alcohol intake, history of CINV and/or pregnancy-related nausea/vomiting, anxiety, expectancy of CINV, chemotherapy cycle number, cancer type, history of morning sickness, performance status, race, comorbidities, use of non-prescription drugs, history of motion sickness, cancer stage, sleeping before chemotherapy, meal before chemotherapy, recent surgery, smoking, neurotransmitter measurements (substance-p and 5hiaa/creatinine), private/public insurance, social functioning, nausea/vomiting before chemotherapy, tumor burden, cancer recurrence (new/recurrent), and symptom distress score.

\subsubsection{Age}

Age was analyzed in 26 studies among which 16 studies confirmed that age is a risk factor of CINV (Table 5). Out of those 16 studies, the results of 15 studies are almost consistent; that is, all of those 15 studies confirmed that the younger patients are at higher risk of CINV than the older patients. However, the term "younger age" was not welldefined in those studies; the threshold for determining "younger age" varied from 40 to 67 in twelve studies, and three studies did not determine any age threshold. The thresholds for 
determining "younger age" are as follows: age $\leq 40(n=3)$, age $\leq 50(n=3)$, age $\leq 55(n=3)$, age $\leq 65(n=2)$, and age $<67(n=1)$ (see Table 4). In contrast, one study showed that older patients (age $\geq 55)$ are at higher risk of CINV [93]. However, in this study, older patients had higher correlation with larger tumor burden (i.e., diameter of the greatest residual tumor $\geq 2 \mathrm{~cm}$ ). They primarily concluded that the patients with larger tumor burden are at higher risk of CINV. Thus, the effect of older age on CINV may be due to its correlation with larger tumor burden. In general, the younger patients are at higher risk of CINV than the older patients.

\subsubsection{Sex}

Gender was analyzed in 18 studies and 11 studies confirmed that female patients are at higher risk of CINV than male patients (see Table 4 and Table 5). One study that initially found female gender at a significantly lower CINV risk in univariate analysis was ultimately found to be insignificant in multivariate analysis and cited the possible explanation as a correlation between gender and other predictive factors (such as age) within the study.

\subsubsection{Alcohol Intake}

The impact of alcohol drinking habit on CINV was studied in 17 studies in which 8 studies confirmed that patients with lower alcohol intake are at higher risk of CINV (see Table 4 and Table 5). Most studies $(n=7)$ defined low alcohol intake on the basis of the number of standard drinks per week. For example, Hilarius et al. (2012) [48], Tamura et al. (2015) [63], Warr et al. (2011) [78], and Hesketh et al. (2010) [81] found that patients taking less than 5 standard drinks per week are at higher risk of CINV than patients taking 
less than 5 standard drinks per week. Both Dranitsaris et al. (2009) [86] and Booth et al. (2007) [89] reported that patients taking at least one standard drinks per day are at lower risk of CINV. In these two studies, low alcohol intake was defined as the number of drinks per week less than 7. Osoba et al. (1997) [92] defined low alcohol intake as the number of drinks per week less than 10; this study also found low alcohol intake to be a significant risk factors for CINV. In contrast, alcohol intake information has been determined based on patients' alcohol consumption habit only without estimating the number of drinks. For example, Sekine et al. (2013) [71] categorized alcohol consumption habit as the habitual vs. non-habitual drinker; this study reported that non-habitual alcohol drinkers are at higher risk of CINV, but did not provide the definition of habitual drinker.

\subsubsection{History of CINV and/or pregnancy-related Nausea/Vomiting}

A total of 17 studies analyzed the history of CINV and/or pregnancy-related nausea/vomiting. Out of those 17 studies, five studies confirmed that patients experiencing CINV during the prior chemotherapy treatment cycles are at higher risk of CINV during their current cycle. One study confirmed the impact of the history of nausea/vomiting on CINV by combining the history of motion sickness, pregnancy-related nausea/vomiting and vestibular dysfunction into a single variable (i.e., history of nausea/vomiting) [70]; history of CINV was not analyzed in that study. Two studies confirmed that female patients experiencing severe nausea/vomiting during their prior pregnancies are ate higher risk of CINV. 


\subsubsection{Anxiety}

Moderate to high anxiety was found to be a risk factor of CINV in 5 studies (out of 8 studies that analyzed that variable); see Table 4 and Table 5. Most of the studies determined the level of anxiety using a Likert scale (e.g., a 4-point Likert scale is graded as none, mild, moderate and high) [96] or a 0-100 mm visual analog scale (VAS) [64]. Yap et al. (2012) [74] studied the impact of 21 anxiety-related symptoms on CINV. They found that 7 symptoms were strongly related to the CINV: fear of dying, fear of the worst, unable to relax, hot/cold sweats, nervousness, faintness, and numbness.

\subsubsection{Expectancy of CINV}

Patients who expect nausea/vomiting after their chemotherapy, regardless of previous experience, are more likely to experience CINV. This attribute has been analyzed in 7 studies out of which 3 studies confirmed the hypothesis.

\subsubsection{Chemotherapy Cycle Number}

This variable has been analyzed in 4 studies in which 2 studies [86,87] demonstrated that patients at earlier cycles (cycle no. < 3) are at higher risk of CINV. A similar result has been reported by Molassiotis et al. (2013) [70] that patients at the first-

cycle of chemotherapy are at higher risk of CINV. However, Shih et al. (2009) [85] found no relation of chemotherapy cycle numbers to the risk of CINV. 


\subsubsection{Comorbidities}

Two out of 5 studies showed that existing comorbidities (such as diabetes, cardiovascular diseases, gastrointestinal, musculoskeletal, thyroid, and other diseases) reduce the risk of CINV [86,89]. In contrast, Bourdeanu et al. (2012) [77] reported that Gastroesophageal Reflux Disease (GERD) increases the risk of CINV, and Furukawa et al. (2014) [66] reported that hypertension was significantly associated with delayed vomiting of grade 1 or higher.

\subsubsection{Cancer Type}

This variable has been analyzed in 12 studies. Only 2 studies found that some specific cancers increase the risk of CINV: breast cancer patients [84] and genitourinary or gynecologic cancer patients [86] were found to be at higher risk of CINV than other cancer types.

\subsubsection{History of Morning Sickness}

Four studies (out of 10 studies that analyzed this variable) found that patients who experienced morning sickness were at higher risk of CINV [63,78,87,89].

\subsubsection{Performance Status (PS)}

The performance status was estimated by the "Eastern Cooperative Oncology Group (ECOG) Performance Status” [97] scale. ECOG performance status is graded at six individual levels (0-5): (1) 0 indicates fully active, (2) 1 indicates ambulatory and able to carry out light works, (3) 2 indicates ambulatory but unable to carry out any works, (4) 3 
indicates capable of only limited self-care, (5) 4 indicates completely disabled, and (6) 5 indicates dead. This variable has been analyzed in 5 studies in which 2 studies reported that poor performance status (i.e. the value of PS is greater than 1 indicating that the patient is not fully active) is a risk factor of CINV [71,95].

\subsubsection{Race}

The impact of race on CINV has been analyzed in 5 studies in which two studies demonstrated that patients from certain races are at higher risk of CINV. Bourdeanu et al. (2012) [77] demonstrated that Asian female breast cancer patients are at higher risk of CINV than Caucasian, African American, and Hispanic patients. Hassan and Yusoff (2010) [80] studied the three races in Malaysia (Malay, Chinese, and Asian Indians) and demonstrated that the Chinese patients were at higher risk of CINV in comparison to the other two ethnicities.

\subsubsection{Use of Non-prescription Drugs}

Two studies demonstrated that the use of non-prescription drugs at home before chemotherapy for nausea/vomiting control significantly increased the risk of CINV $[86,87]$. Both of the studies analyzed the same dataset separately for the acute and delayed phase of CINV. The names of the non-prescription drugs were not reported in those studies.

\subsubsection{History of Motion Sickness}

Two out of 17 studies demonstrated that the history of motion sickness is a risk factor of CINV [85]. Motion sickness is defined as sickness that is caused due to motion while traveling by car, train, airplanes or boats. 


\subsubsection{Cancer Stage}

There are five stages in cancer: stage 0, I, II, III, and IV. The variable "cancer stage" has been analyzed in six studies but only one study reported that patients with early stages of cancer (stage I and II) are at higher risk of CINV during the acute phase [86]; the reasoning behind this fact was not discussed in this study. In contrast, cancer stage was reported to be not significant for developing CINV during the delayed phase using the same dataset [87].

\subsubsection{Sleeping before Chemotherapy}

Petrella et al. (2009) [87] reported that with the increase of the number of hours of sleep in the night before the chemotherapy, the probabilities of CINV during delayed phase decreased. In contrast, this variable had no significant impact on developing CINV during acute phase using the same dataset [96]. There are two other studies that analyzed the variable, but found no impact on developing CINV (see Table 4).

\subsubsection{Meal before Chemotherapy}

Booth et al. (2007) [89] reported that having no food before chemotherapy increased the probability of CINV. As such, this study suggested that the importance of eating a small amount of food prior to treatment should be emphasized during prechemotherapy education so that the risk of developing CINV could be reduced. Three other studies analyzed this attribute, but found no impact on CINV (see Table 4). 


\subsubsection{Recent Surgery}

Booth et al. (2007) [89] reported that having surgery within the past three months of chemotherapy increased the probability of CINV. Two other studies analyzed this attribute as well, but found no impact on CINV (see Table 4).

\subsubsection{Smoking History}

Two studies conducted in Japan by Sekine et al. (2013) [71] and Furukawa et al. (2014) [66] analyzed the impact of smoking on CINV. Among those studies, only the study by Sekine et al. (2013) [71] reported that non-smokers are at higher risk of CINV than smokers.

\subsubsection{Neurotransmitter Measurements}

Substance-P and 5-HIAA/creatinine are two neurotransmitter measurements that are measured from blood and urine sample respectively. Higa et al. (2012) [98] studied the correlation of these neurotransmitters with the development of CINV due to moderately emetogenic chemotherapy (MEC). This study reported that the ratio of substance-P to 5HIAA/creatinine greater than 70 is significantly associated with the development of CINV during delayed phase due to MEC.

\subsubsection{Private/Public Insurance}

Bourdeanu et al. (2012) [77] performed a study in USA on breast cancer patients and demonstrated that patients having private health insurances are at higher risk of CINV than the ones having public health insurances such as state or federally funded insurances. 


\subsubsection{Social Functioning}

Social functioning is a variable within the health-related quality-of-life (HQL) indicator. Osoba et al. (1997) [92] reported that a social functioning score less than 70 is a significant predictor of CINV. The social functioning score is measured by the European Organization for Research and Treatment (EORTC) Core Quality of Life Questionnaire (QLQ-C30) [99]. The score ranges from 0 to 100, and a higher score indicates that the level of functioning is higher.

\subsubsection{Nausea/Vomiting Before Chemotherapy}

Petrella et al. (2009) [87] demonstrated that nausea/vomiting before chemotherapy is a significant predictor of CINV during the delayed phase, but not in the acute phase [86]. Molassiotis et al. (2014) [64] also supported that nausea prior to chemotherapy was a predictor of CINV, but this finding was significant for both the delayed and the acute phases.

\subsubsection{Tumor Burden}

Tumor burden/load is indicative of the amount of cancer in the body. Hursti et al. (1996) [93] categorized the tumor burden into two groups: (1) minimal tumor burden was defined as the diameter of the greatest residual tumor being less than 2 centimeters, and (2) large tumor burden was defined as the diameter of the greatest residual tumor being greater or equal to 2 centimeters. This study reported that the ovarian cancer patients with larger tumor burdens are at higher risk of CINV. 


\subsubsection{Cancer Recurrence (new/recurrent)}

du Bois et al. (1992) [94] demonstrated that recurrent cancer patients are at higher risk of CINV than new cancer patients. This variable is related to the history of chemotherapy since patients with recurrent cancer likely went through more chemotherapy in the past.

\subsubsection{Symptom Distress Score}

Molassiotis et al. (2013) [70] used Symptom Distress Score [100] to measure the symptoms present before chemotherapy. This scale measures the symptom distress for a total of 13 items such as nausea, appetite, insomnia, pain, fatigue, bowel pattern, concentration, appearance, outlook, breathing, cough, frequency of nausea, and frequency of pain. Molassiotis et al. (2013) [70] reported that out of these 13 items, only pain was linked with the development of CINV.

\subsubsection{Non-significant Factors}

Table 6 presents a total of 16 patient-related factors that were not found to be significantly related to the prediction of CINV in any study. These factors are history of previous chemo $(\mathrm{n}=6)$, depression, $(\mathrm{n}=1)$, education $(\mathrm{n}=2)$, marital status $(\mathrm{n}=1)$, religion $(\mathrm{n}=1)$, weight $(\mathrm{n}=1)$, BMI $(\mathrm{n}=2)$, frequency of chemotherapy cycle $(\mathrm{n}=1)$, number of risk factors $(n=1)$, drowsiness $(n=1)$, neuroticism $(n=1)$, autonomic perception $(n=1)$, menopause $(\mathrm{n}=1)$, defecations (bowel movement) $(\mathrm{n}=1)$, history of radiotherapy $(\mathrm{n}=1)$, and ascites $(n=1)$. 
Table 6: Insignificant Patient-Related Factors

\begin{tabular}{|l|l|}
\hline Attribute Name & \# of Studies \\
\hline History of Previous Chemo & 6 \\
\hline Depression & 1 \\
\hline Education & 2 \\
\hline Marital Status & 1 \\
\hline Religion & 1 \\
\hline Weight & 1 \\
\hline BMI & 2 \\
\hline Frequency of chemotherapy cycle & 1 \\
\hline Number of risk factors & 1 \\
\hline Drowsiness & 1 \\
\hline Neuroticism & 1 \\
\hline Autonomic perception & 1 \\
\hline Menopause & 1 \\
\hline Defecations (bowel movement) & 1 \\
\hline History of radiotherapy & 1 \\
\hline Ascites & 1 \\
\hline & \\
\hline
\end{tabular}

\subsection{DISCUSSIONS}

A significant amount of research has been performed to identify the patient-related risk factors of CINV. We have identified 30 studies through a systematic literature search strategy. These studies included a total of 42 patient-related variables in which 26 factors were found to have significant impact on the risk of CINV. Three factors are demographicrelated: younger patients, female gender, and Asian race. A total of sixteen factors are innate to patient's physiology or influenced by physiology, and may alter the pathophysiology of CINV (i.e., intrinsic); these factors include history of CINV or pregnancy-related nausea/vomiting, anxiety, expectancy of CINV, absence of 
comorbidities, cancer type (i.e., breast, genitourinary or gynecologic cancer), history of morning sickness, poor performance status, history of motion sickness, early stage of cancer, lower number of hours of sleep before chemotherapy, recent surgery within the past three months, a higher ratio of neurotransmitter measurements, nausea/vomiting before chemotherapy, larger tumor burden, recurrent cancer, and higher symptom distress score. Seven factors may influence the pathophysiology of CINV that are external in nature (i.e., extrinsic); these factors include low alcohol intake, patients at earlier cycles $(\leq 3)$ of chemotherapy, use of non-prescription drugs at home for emesis control before chemotherapy, no food intake before chemotherapy, non-smoking habit, having private insurance, and low social functioning.

None of the studies considered all 42 variables simultaneously. The average number of variables included per study was 7.06 with a minimum of 1 and a maximum of 16 (median $=6$ and standard deviation $=4.18)$. The average number of significant risk factors that were reported per study was 2.7 with a minimum of 0 and a maximum of 7 $($ median $=2$ and standard deviation $=1.99)$. The maximum number of patient-related risk factors that were reported by a single study was $7(n=3)$ followed by $5(n=3)$ and $4(n=2)$. Dranitsaris et al. (2009) [86], Petrella et al. (2009) [87]and Booth et al. (2007) [89] reported 7 risk factors. Tamura et al. (2015) [63], Molassiotis et al. (2014)[64], and Molassiotis et al. (2013) [70] reported 5 risk factors. Sekine et al. (2013) [71] and Osoba et al. (1997) [92] reported 4 risk factors. 


\subsection{SIGNIFICANCE OF THIS SYSTEMATIC REVIEW}

The significance of this study is threefold. First, the current CINV guidelines that are basically based on antiemetics for the risk of CINV can be augmented using the findings from this review (i.e., a comprehensive list of significant patient-related factors) so that oncologists can consider these factors when they predict CINV. Second, the findings of the study can be used to discover associations among those patient-related factors. While it is important to identify individual patient-related factors, the more important research may be to discover how those individual factors are associated one another in terms of the risk of CINV. Third, this review may lay the foundation for the construction of CINV prediction models. Currently, CINV prediction models based on antiemetics and patientrelated factors are unsatisfactory $[70,86,87,96,101]$. This indicates that associations among patient-related factors are not fully identified, and/or there are some important, yet hidden, patient-related factors.

\subsection{LIMITATIONS}

We have identified four limitations of this systematic review study. First, only two studies included the low emetogenic chemotherapy (LEC) in conjunction with the high and moderate emetogenic chemotherapy. None of the studies independently analyzed patient related risk factors for LEC. Thus, the literature does not confirm whether patient-related factors have an impact on CINV due to LEC. Second, the number of subjects in the studies are not large enough; half of the studies employed less than 243 subjects. The average number of subjects per study was 476.7 with a minimum of 25 and a maximum of 1,910 (standard deviation=504.7). Third, the methods for determining the significance level of 
risk factors were not consistent across different studies. Fourth, the cut-off/threshold for numerical variables were not similar across different studies. For example, five different age cut-offs were used for determining younger patients who are at risk for developing CINV.

\subsection{CONCLUSION}

This systematic review study has identified and summarized patient-related factors that significantly impact a patient's risk of CINV. The identification of patients at risk for CINV based on key risk factors prior to the initiation of a chemotherapy regimen is imperative. Oncologists may be able to selectively focus on more aggressive treatments on those high risk patients. Although there are 30 significant risk factors (see Table 5), most studies used only two to three significant factors as well as many non-significant factors, as discussed in the discussion section. Therefore, future studies are needed that include as many significant risk-factors as possible to see how, in combinations, they may affect the risk of CINV. 


\section{CHAPTER 3 : CLINICAL DATA}

\subsection{DATA SOURCES}

This was a single-center retrospective study. The data were collected by retrospective record review from the electronic medical records used at the University of Missouri Ellis Fischel Cancer Center. The study was approved by the MU Health Sciences IRB (project number 1208541).

\subsection{INCLUSION AND EXCLUSION CRITERIA}

The patients who received chemotherapy and standard antiemetic prophylaxis (based on national antiemetic guidelines) at the oncology outpatient service during the period of June 1, 2010 to July 31, 2012 were included in the study. The patients, who were treated with concurrent radiotherapy or surgical procedure, were excluded from the study. Also, the patients with missing information were excluded from the study.

\subsection{DATA COLLECTION PLAN}

The first aim of this project was to collect data. There are two phases of CINV: acute phase and delayed phase. We planned to collect two independent datasets for each phases of CINV. The rationale behind collecting two independent datasets was that the pathophysiology of CINV for acute phase is different than the delayed phase. The 
pathophysiology of CINV is a complex process (see section 1.3). Since the acute and delayed CINV follows two different pathophysiology, we plan to discover the patientrelated risk factors for causing CINV during both phases independently. As such, two independent datasets were needed.

In each dataset, there are three groups of data based on the emetogenecity of chemotherapy regimens. The degree of emetogenecity of chemotherapy regimens is divided into four emetic risk categories: minimal, low, moderate, and high (see section 1.4). The minimal risk category of chemotherapy for causing CINV is not clinically important since only less than $10 \%$ of patients suffers from CINV. Thus, we collected data in three groups corresponding to three clinically important categories: low, moderate and high.

\subsection{CLASS BALANCING}

The dataset may suffer from class-imbalance problem for a group of data. For example, less than $30 \%$ patients suffer from CINV due to low emetogenic chemotherapy (LEC). With the use of standard antiemetic treatment, this percentage will be further reduced. As such, the dataset will have far less number of CINV cases than no-CINV cases. Another example is the high emetogenic chemotherapy (HEC) group where the number of CINV cases are very higher than the no-CINV cases. Our major classes of interest includes both CINV and no-CINV cases. As a result, class balancing in each group of data (LEC, MEC and HEC) was deemed necessary. This was achieved by making the size of dataset in each class for each group approximately equal. 


\subsection{VARIABLE SELECTION}

In order to identify the potential patient-related variables for causing CINV, we have completed a systematic review study by following the "Preferred Reporting Items for Systematic Reviews and Meta-Analyses (PRISMA)" guideline [61]. Chapter 2 presents the results of the study. We searched MEDLINE to identify the articles that demonstrated patient-related risk factors of CINV through clinical studies. A total of 30 articles were selected for this study. A total of 26 patient-related risk-factors were documented. In this project, we included 13 independent variables and 1 dependent variable (CINV outcome) based on the recommendations from the chemotherapy experts in the MU Elis Fischel Cancer Center; see Table 7 for data descriptions. The rationale behind choosing those 13 variables is two-fold. First, the experts consider some of those variables as clinically significant for causing CINV based on their experience. Second, those variables are easy to collect during a clinical encounter. This is important because the prediction model should be readily usable at the point-of-care before chemotherapy. 
Table 7: Patient-Related Variables

\begin{tabular}{|c|c|}
\hline Variable Name & Description and Data Domain \\
\hline Age & Age of the patient \\
\hline Sex & Male or Female \\
\hline Race & Asian, Hispanic, White, African American \\
\hline $\begin{array}{l}\text { Body Mass Index } \\
\text { (BMI) }\end{array}$ & Calculated based on the height and weight of the patient \\
\hline Type of cancer & $\begin{array}{l}\text { Solid Cancers: } \\
-\quad \text { Breast cancer } \\
-\quad \text { Lung cancer } \\
-\quad \text { Colorectal and anal cancer } \\
-\quad \text { Genitourinary cancer } \\
-\quad \text { Other solid cancers } \\
\text { Hematological Cancers: } \\
-\quad \text { Leukemia both acute and chronic } \\
-\quad \text { Myeloma } \\
-\quad \text { Lymphoma both acute and chronic } \\
-\quad \text { Other heme malignancies }\end{array}$ \\
\hline Stage of Cancer & $\begin{array}{l}\text { The severity of a person's cancer based on the extent of the original (primary) } \\
\text { tumor and whether or not cancer has spread in the body. There are five stages: } \\
\text { 0, I, II, III, and IV. }\end{array}$ \\
\hline $\begin{array}{l}\text { Number of } \\
\text { Comorbidities }\end{array}$ & $\begin{array}{l}\text { Up to four comorbidities are considered: } \\
-\quad \text { Cardiovascular diseases: heart diseases/coronary artery diseases, high } \\
\text { blood pressure, high cholesterol, atrial fibrillation, or any cardiac } \\
\text { arrhythmia } \\
-\quad \text { Respiratory system diseases: asthma, COPD, or chronic bronchitis, etc. } \\
-\quad \text { Chronic pain syndrome } \\
-\quad \text { Other diseases: moderate to severe Gastroesophageal reflux } \\
\text { disease (GERD), crohn's disease, any chronic gallbladder, any chronic } \\
\text { pancreatic problem, chronic diarrhea/constipation, or any moderate to } \\
\text { severe psychiatric illnesses. }\end{array}$ \\
\hline Alcohol Consumption & $\begin{array}{l}\text { This attribute describes the alcohol drinking habit of the patient. The patients } \\
\text { having two or less standard drinks per week are considered as low alcohol } \\
\text { drinking habit; otherwise, the patients are considered as high alcohol drinking } \\
\text { habit. }\end{array}$ \\
\hline Current smoker & $\begin{array}{l}\text { This attribute describes the smoking habit of the patient. This is mainly } \\
\text { characterized by the nicotine dependence that can occur with cigarette smoking, } \\
\text { smokeless tobacco use and/or Cigar or pipe use. A current smoker is defined as } \\
\text { the person had any nicotine dependence in last } 30 \text { days. }\end{array}$ \\
\hline Anxiety & $\begin{array}{l}\text { This attribute describes whether the patient had anxiety during treatment. This } \\
\text { has been determined from the psychosocial evaluation report by a social worker } \\
\text { or a physician. }\end{array}$ \\
\hline Dehydration & $\begin{array}{l}\text { This attribute describes whether the patient had dehydration or not before the } \\
\text { chemotherapy }\end{array}$ \\
\hline $\begin{array}{l}\text { Number of prior } \\
\text { chemotherapy } \\
\text { regimens }\end{array}$ & $\begin{array}{l}\text { This attribute describes the number of different chemotherapy regimens that has } \\
\text { been used for a patient previously. A chemotherapy regimen consists of multiple } \\
\text { cycles of same chemotherapy. }\end{array}$ \\
\hline $\begin{array}{l}\text { History of previous } \\
\text { CINV }\end{array}$ & $\begin{array}{l}\text { This attribute describes whether the patient had at least grade } 2 \text { to } 4 \text { CINV in } \\
\text { their last cycle of chemotherapy. }\end{array}$ \\
\hline $\begin{array}{l}\text { Symptoms }(\mathrm{Sx}) / \mathrm{CINV} \\
\text { Outcome }\end{array}$ & $\begin{array}{l}\text { This attribute describes the use of antiemetic outcome. The use of antiemetics } \\
\text { is based on the national guidelines. The patients suffering from grade } 2 \text { or more } \\
\text { severe CINV with the use of guideline-based antiemetics are considered as } \\
\text { CINV positive; otherwise, the patients are considered as CINV negative. }\end{array}$ \\
\hline
\end{tabular}




\subsection{ELECTRONIC DATA CAPTURE}

The MU-iCATS's Research Informatics Core was used as a central location for data processing and management. The MU-iCATS has implemented an electronic data capture system called Research Electronic Data Capture (REDCap) [102,103], which was developed by Vanderbilt University. Figure 4 presents the REDCap data collection instrument for this project. The REDCap data collection instrument consists of both numerical coding and text labels in the data entry forms for 14 variables including the class variable. This resolved the issue of data coding error. For text entry of numerical data, validation rules were applied. Both datasets were verified by an oncologist for clinical correctness. 


\begin{tabular}{|c|c|c|}
\hline Record ID & & 3054 \\
\hline Age & (H) & \\
\hline "must provide value & $x$ & Age of the patient during chemotherapy \\
\hline $\begin{array}{l}\text { Sex } \\
\text { " must provide value }\end{array}$ & (H) & Male Female \\
\hline $\begin{array}{l}\text { Race } \\
\text { "must provide value }\end{array}$ & (H) & $\begin{array}{l}\text { Asian } \\
\text { Hispanic } \\
\text { White } \\
\text { African American }\end{array}$ \\
\hline Body Mass Index (BMI) & (H) & \\
\hline "must provide value & 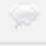 & BMI during chemotherapy \\
\hline $\begin{array}{l}\text { Type of Cancer } \\
\text { must provide value }\end{array}$ & (H) & $\begin{array}{l}\text { Breast Cancer } \\
\text { Lung Cancer } \\
\text { Colorectal and Anal Cancer } \\
\text { Genitourinary Cancer } \\
\text { Other Solid Cancers } \\
\text { Leukemia both Acute and Chronic } \\
\text { Myeloma } \\
\text { Lymphoma both Acute and Chronic } \\
\text { Other Heme Malignancies }\end{array}$ \\
\hline $\begin{array}{l}\text { Stage of Cancer } \\
\text { must provide value }\end{array}$ & (4) & $\begin{array}{l}\text { Stage } 0 \\
\text { Stage I } \\
\text { Stage II } \\
\text { Stage III } \\
\text { Stage IV }\end{array}$ \\
\hline $\begin{array}{l}\text { Number of Comorbidities } \\
\text { " must provide value }\end{array}$ & $(H)$ & $\bigcirc_{1} \bigcirc 2 \bigcirc 3 \bigcirc 4$ \\
\hline $\begin{array}{l}\text { Alcohol Consumption } \\
\text { "must provide value }\end{array}$ & (H) & $\begin{array}{l}\text { The patient have LOW (two or less drinks per week) or } \\
\text { NO alcohol consumption habit } \\
\text { The patient consumes more than two drinks per week }\end{array}$ \\
\hline $\begin{array}{l}\text { Is the Patient a Current Smoker? } \\
\text { "must provide value }\end{array}$ & (H) & $\begin{array}{l}\text { The Patient is NOT a current smoker } \\
\text { The patient is a current smoker }\end{array}$ \\
\hline $\begin{array}{l}\text { Anxiety } \\
\text { " must provide value }\end{array}$ & (H) & $\begin{array}{l}\text { The Patient had NO Anxiety during Chemotherapy } \\
\text { The Patient had Anxiety during Chemotherapy }\end{array}$ \\
\hline $\begin{array}{l}\text { Dehydration } \\
\text { "must provide value }\end{array}$ & (H) & $\begin{array}{l}\text { The Patient had NO Dehydration before Chemotherapy } \\
\text { The Patient had Dehydration before Chemotherapy }\end{array}$ \\
\hline $\begin{array}{l}\text { Number of Prior Chemotherapy Regimen } \\
\text { " must provide value }\end{array}$ & (H) & $\begin{array}{l}\text { Number of different chemotherapy regimen that has been used for a } \\
\text { patient previously. A chemotherapy regimen consists of multiple } \\
\text { cycles of same chemotherapy }\end{array}$ \\
\hline $\begin{array}{l}\text { Emetogenecity of Chemotherapy } \\
\text { must provide value }\end{array}$ & $($ (H) & $\begin{array}{l}\text { Low Emetogenic Chemotherapy (LEC) } \\
\text { Moderate Emetogenic Chemotherapy (MEC) } \\
\text { High Emetogenic Chemotherapy (HEC) }\end{array}$ \\
\hline $\begin{array}{l}\text { History of Previous CINV } \\
\text { must provide value }\end{array}$ & $($ (H) & $\begin{array}{l}\text { The Patient had NO CINV (Grade } 2 \text { to } 4 \text { ) during the } \\
\text { Last Cycle of Chemotherapy } \\
\text { The patient had CINV (Grade } 2 \text { to } 4 \text { ) During the Last } \\
\text { Cycle of Chemotherapy }\end{array}$ \\
\hline $\begin{array}{l}\text { Symptoms (Sx)/CINV Outcome } \\
\text { " must provide value }\end{array}$ & (H) & $\begin{array}{l}\text { The Patient had less than Grade } 2 \text { CINV } \\
\text { The Patient had at least Grade } 2 \text { or more Severe CINV }\end{array}$ \\
\hline
\end{tabular}

Figure 4: REDCap Data Collection Instrument 


\subsection{DATA SUMMARY}

A total of 6,124 records were collected based on the inclusion and exclusion criteria described in section 3.2; the acute phase dataset contains 3,053 records and the delayed phase dataset contains 3,071 records. Table 8 and Table 9 presents the breakdown of both datasets in terms of three chemotherapy categories and two treatment outcomes. Table 10 and Table 11 presents the overview of both acute and delayed phase dataset, consecutively.

Table 8: Acute Phase Data Summary

\begin{tabular}{|l|c|c|c|}
\hline \multicolumn{1}{|c|}{ CINV Treatment Group } & \# of records & CINV=Yes & CINV=No \\
\hline High Emetogenic Chemo (HEC) & 1,026 & $504(49.12 \%)$ & $522(50.88 \%)$ \\
\hline Moderate Emetogenic Chemo (MEC) & 1,012 & $506(50 \%)$ & $506(50 \%)$ \\
\hline Low Emetogenic Chemo (LEC) & 1,015 & $506(50.15 \%)$ & $509(49.85 \%)$ \\
\hline Grand Total & 3,053 & $1,519(49.75 \%)$ & $1,534(50.25 \%)$ \\
\hline
\end{tabular}

Table 9: Delayed Phase Data Summary

\begin{tabular}{|l|c|c|c|}
\hline \multicolumn{1}{|c|}{ CINV Treatment Group } & \# of records & CINV=Yes & CINV=No \\
\hline High Emetogenic Chemo (HEC) & 1,166 & $586(50.26 \%)$ & $580(49.74 \%)$ \\
\hline Moderate Emetogenic Chemo (MEC) & 891 & $447(50.17 \%)$ & $444(49.83 \%)$ \\
\hline Low Emetogenic Chemo (LEC) & 1,014 & $519(51.18 \%)$ & $495(48.82 \%)$ \\
\hline Grand Total & 3,071 & $1,552(50.54 \%)$ & $1,519(49.46 \%)$ \\
\hline
\end{tabular}

Table 10: Acute Phase Data Overview

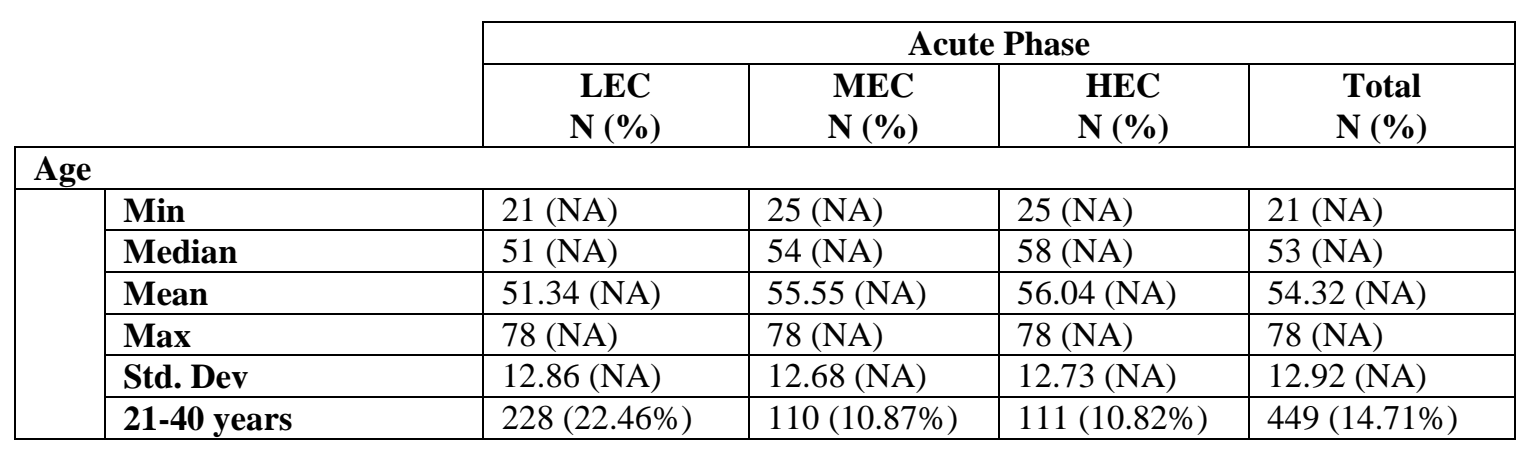




\begin{tabular}{|c|c|c|c|c|c|}
\hline & 41-60 years & $507(49.95 \%)$ & $515(50.89 \%)$ & $496(48.34 \%)$ & $1,518(49.72 \%)$ \\
\hline & 61-80 years & $280(27.59 \%)$ & $387(38.24 \%)$ & $419(40.84 \%)$ & $1,086(35.57 \%)$ \\
\hline \multicolumn{6}{|c|}{ BMI } \\
\hline & Min & 19 (NA) & 19 (NA) & 19 (NA) & 19 (NA) \\
\hline & Max & 29 (NA) & 30 (NA) & 29 (NA) & 29 (NA) \\
\hline & Mean & $30.03(\mathrm{NA})$ & $30.25(\mathrm{NA})$ & $29.93(\mathrm{NA})$ & 30.07 (NA) \\
\hline & Median & 39 (NA) & 39 (NA) & 39 (NA) & 39 (NA) \\
\hline & Std. Dev & 4.77 (NA) & 4.77 (NA) & 4.86 (NA) & 4.80 (NA) \\
\hline & $\begin{array}{l}\text { Underweight } \\
\text { (Below 18.5) }\end{array}$ & $0(00.00 \%)$ & $0(00.00 \%)$ & $0(00.00 \%)$ & $0(00.00 \%)$ \\
\hline & $\begin{array}{l}\text { Normal } \\
(18.5-\text { Below 25) }\end{array}$ & $88(08.67 \%)$ & $75(07.41 \%)$ & $101(09.84 \%)$ & $264(08.65 \%)$ \\
\hline & $\begin{array}{l}\text { Overweight } \\
(25.0-\text { Below 30) }\end{array}$ & $427(42.07 \%)$ & $422(41.70 \%)$ & $431(42.01 \%)$ & $1,280(41.93 \%)$ \\
\hline & $\begin{array}{l}\text { Obese } \\
\text { (30.0 and Above) }\end{array}$ & $500(49.26 \%)$ & $515(50.89 \%)$ & $494(48.15 \%)$ & $1,509(49.43 \%)$ \\
\hline \multicolumn{6}{|c|}{ Number of Comorbidity } \\
\hline & 1 & $253(24.93 \%)$ & $300(29.64 \%)$ & $399(38.89 \%)$ & $952(31.18 \%)$ \\
\hline & 2 & $464(45.71 \%)$ & $469(46.34 \%)$ & $402(39.18 \%)$ & $1,335(43.73 \%)$ \\
\hline & 3 & $95(09.36 \%)$ & $90(08.89 \%)$ & $113(11.01 \%)$ & $298(09.76 \%)$ \\
\hline & 4 & $203(20.00 \%)$ & $153(15.12 \%)$ & $112(10.92 \%)$ & $468(15.33 \%)$ \\
\hline \multicolumn{6}{|c|}{ Gender } \\
\hline & Female & $611(60.20 \%)$ & $502(49.60 \%)$ & $539(52.53 \%)$ & $1,652(54.11 \%)$ \\
\hline & Male & $404(39.80 \%)$ & $510(50.40 \%)$ & $487(47.47 \%)$ & $1401(45.89 \%)$ \\
\hline \multicolumn{6}{|c|}{ Race } \\
\hline & Asian & $69(06.80 \%)$ & $77(07.61 \%)$ & $58(05.65 \%)$ & $204(06.68 \%)$ \\
\hline & African American & $178(17.54 \%)$ & $190(18.77 \%)$ & $164(15.98 \%)$ & $532(17.43 \%)$ \\
\hline & Hispanic & $71(07.00 \%)$ & $73(07.21 \%)$ & $68(06.63 \%)$ & $212(06.94 \%)$ \\
\hline & White & $697(68.67 \%)$ & $672(66.40 \%)$ & $736(71.73 \%)$ & $2,105(68.95 \%)$ \\
\hline \multicolumn{6}{|c|}{ Stage of Cancer } \\
\hline & Stage I & $151(14.88 \%)$ & $224(22.13 \%)$ & $111(10.82 \%)$ & $486(15.92 \%)$ \\
\hline & Stage II & $111(10.94 \%)$ & $160(15.81 \%)$ & $79(07.70 \%)$ & $350(11.46 \%)$ \\
\hline & Stage III & $467(46.01 \%)$ & $385(38.04 \%)$ & $507(49.42 \%)$ & $1,359(44.51 \%)$ \\
\hline & Stage IV & $286(28.18 \%)$ & $243(24.01 \%)$ & $329(32.07 \%)$ & $858(28.10 \%)$ \\
\hline \multicolumn{6}{|c|}{ Alcohol Consumption } \\
\hline & Low & $567(55.86 \%)$ & $540(53.36 \%)$ & $290(28.27 \%)$ & $1,397(45.76 \%)$ \\
\hline & High & $448(44.14 \%)$ & $472(46.64 \%)$ & $736(71.73 \%)$ & $1,656(54.24 \%)$ \\
\hline \multicolumn{6}{|c|}{ Smoking Status } \\
\hline & Not a Current Smoker & $501(49.36 \%)$ & $396(39.13 \%)$ & $481(46.88 \%)$ & $1,378(45.14 \%)$ \\
\hline & Current Smoker & $514(50.64 \%)$ & $616(60.87 \%)$ & $545(53.12 \%)$ & $1,675(54.86 \%)$ \\
\hline \multicolumn{6}{|c|}{ Dehydration } \\
\hline & No & $507(49.95 \%)$ & $518(51.19 \%)$ & $568(55.36 \%)$ & $1,593(52.18 \%)$ \\
\hline & Yes & $508(50.05 \%)$ & $494(48.81 \%)$ & $458(44.64 \%)$ & $1,460(47.82 \%)$ \\
\hline \multicolumn{6}{|c|}{ Anxiety } \\
\hline & No & $462(45.52 \%)$ & $520(51.38 \%)$ & $469(45.71 \%)$ & $1,451(47.53 \%)$ \\
\hline & Yes & $553(54.48 \%)$ & $492(48.62 \%)$ & $557(54.29 \%)$ & $1,602(52.47 \%)$ \\
\hline \multicolumn{6}{|c|}{ Number of Previous Chemotherapy } \\
\hline & $\mathbf{0}$ & $406(40.00 \%)$ & $386(38.14 \%)$ & $263(25.63 \%)$ & $1,055(34.56 \%)$ \\
\hline & 1 & $259(25.52 \%)$ & $329(32.51 \%)$ & $470(45.81 \%)$ & $1,058(34.65 \%)$ \\
\hline & 2 & $178(17.54 \%)$ & $229(22.63 \%)$ & $201(19.59 \%)$ & $608(19.91 \%)$ \\
\hline & 3 & $113(11.13 \%)$ & $68(06.72 \%)$ & $92(08.97 \%)$ & $273(08.94 \%)$ \\
\hline & 4 & $59(05.81 \%)$ & $0(00.00 \%)$ & $0(00.00 \%)$ & $59(01.93 \%)$ \\
\hline \multicolumn{6}{|c|}{ Prior CINV } \\
\hline & No & $516(50.84 \%)$ & $432(42.69 \%)$ & $569(55.46 \%)$ & $1,517(49.69 \%)$ \\
\hline
\end{tabular}




\begin{tabular}{|c|c|c|c|c|c|}
\hline & Yes & $499(49.16 \%)$ & $580(57.31 \%)$ & $457(44.54 \%)$ & $1,536(50.31 \%)$ \\
\hline \multicolumn{6}{|c|}{ Type of Cancer } \\
\hline \multicolumn{6}{|r|}{$231(07.57 \%)$} \\
\hline & Lung & $140(13.79 \%)$ & $144(14.23 \%)$ & $150(14.62 \%)$ & $434(14.22 \%)$ \\
\hline & Colorectal \& anal & $89(08.77 \%)$ & $82(08.10 \%)$ & $86(08.38 \%)$ & $257(08.42 \%)$ \\
\hline & Genitourinary & $119(11.72 \%)$ & $131(12.94 \%)$ & $113(11.01 \%)$ & $363(11.89 \%)$ \\
\hline & Other solid cancer & $84(08.28 \%)$ & $65(06.42 \%)$ & $78(07.60 \%)$ & $227(07.44 \%)$ \\
\hline & $\begin{array}{l}\text { Leukemia both acute } \\
\text { and chronic }\end{array}$ & $152(14.98 \%)$ & $162(16.01 \%)$ & $151(14.72 \%)$ & $465(15.23 \%)$ \\
\hline & Myeloma & $120(11.82 \%)$ & $99(09.78 \%)$ & $113(11.01 \%)$ & $332(10.87 \%)$ \\
\hline & $\begin{array}{l}\text { Lymphoma both acute } \\
\text { and chronic }\end{array}$ & $134(13.20 \%)$ & $140(13.83 \%)$ & $148(14.42 \%)$ & $422(13.82 \%)$ \\
\hline & $\begin{array}{l}\text { Other heme } \\
\text { malignancies }\end{array}$ & $105(10.34 \%)$ & $104(10.28 \%)$ & $113(11.01 \%)$ & $322(10.55 \%)$ \\
\hline \multicolumn{6}{|c|}{ CINV Outcome } \\
\hline & No CINV & $506(49.85 \%)$ & $506(50.00 \%)$ & $522(50.88 \%)$ & $1,534(50.25 \%)$ \\
\hline & CINV & $509(50.15 \%)$ & $506(50.00 \%)$ & $504(49.12 \%)$ & $1,519(49.75 \%)$ \\
\hline
\end{tabular}

Table 11: Delayed Phase Data Overview

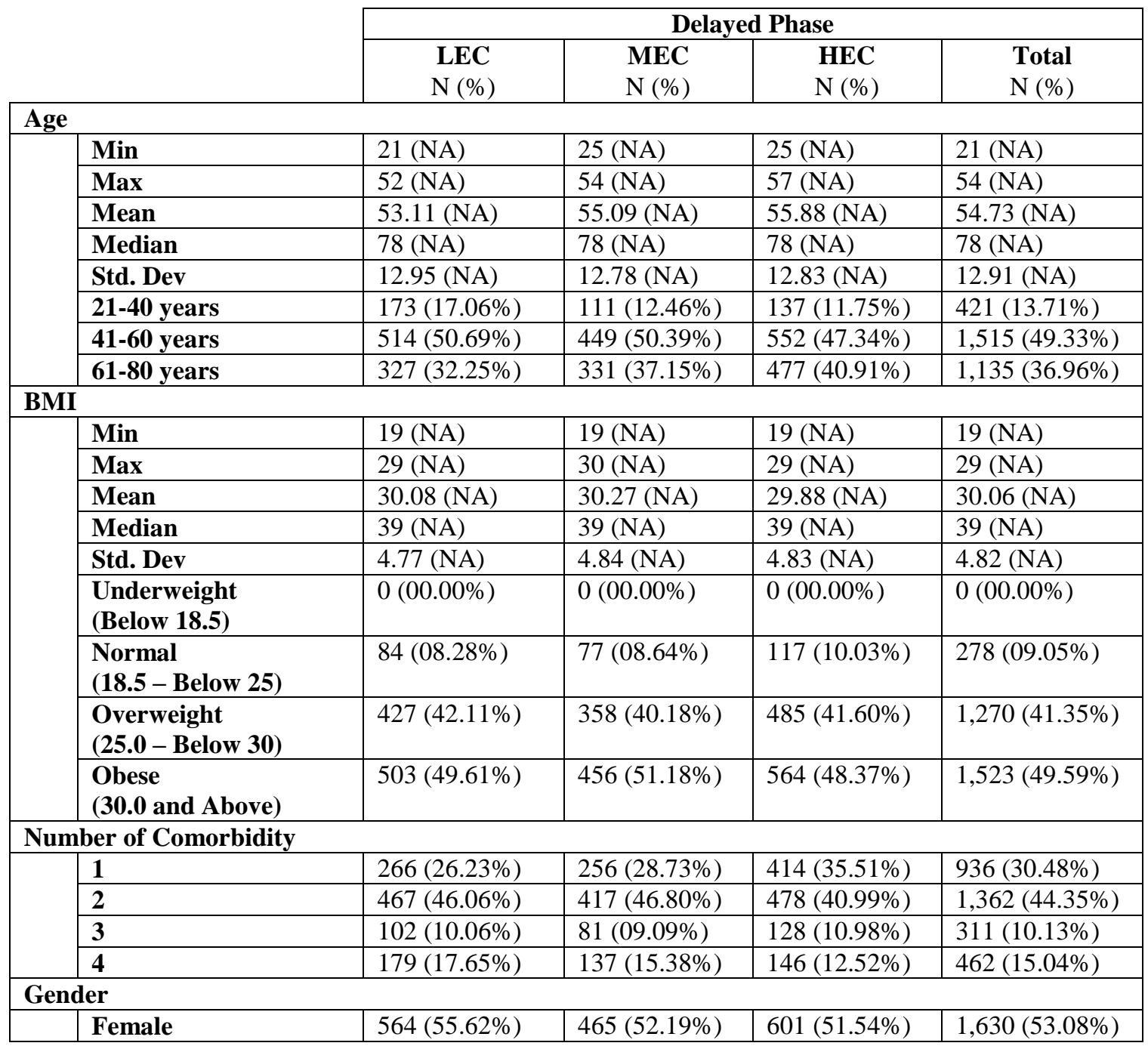




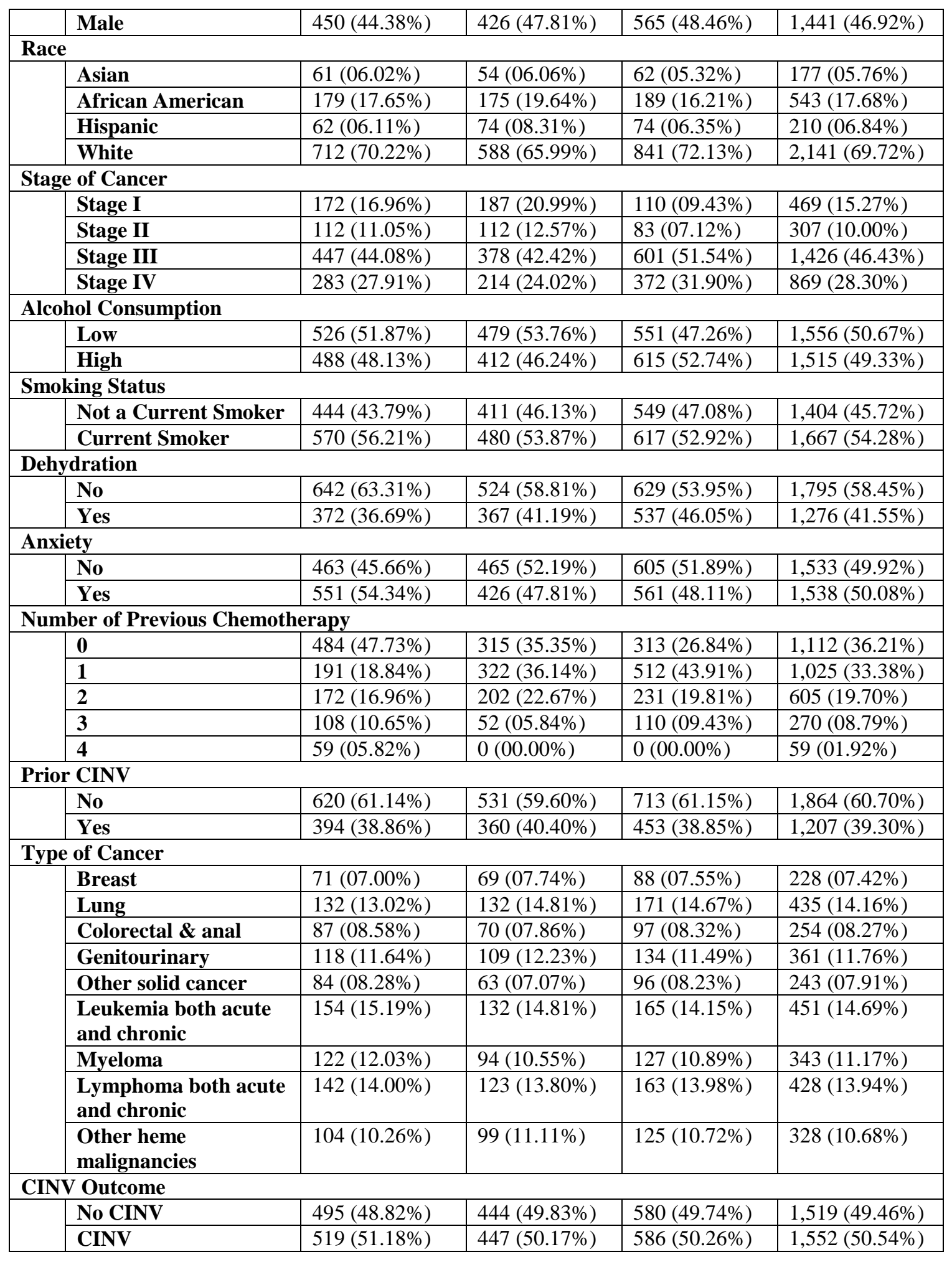




\subsection{DATA CHARACTERISTICS}

This section describes the characteristics of each data domains in terms of their predictive nature (i.e., the probability of CINV) for three treatment categories and two phases of CINV.

\subsubsection{Age vs. CINV Outcome}

In our systematic review, we have found that younger patients were at higher risk of CINV. However, age cutoff was not well-defined; the threshold for determining younger age varied from 40 to 67 . Figure 5 presents the probability of CINV against age. The probability of CINV varied only for low emetogenic chemotherapy (LEC) in acute and delayed phase; younger patients had higher chance of CINV which is consistent with the literature. From the graph, we observe that patients less than 40 years old were at higher risk of CINV for LEC. For moderate and high emetogenic chemotherapies, the probability of CINV was about $50 \%$ for any ages for both acute and delayed phases. 


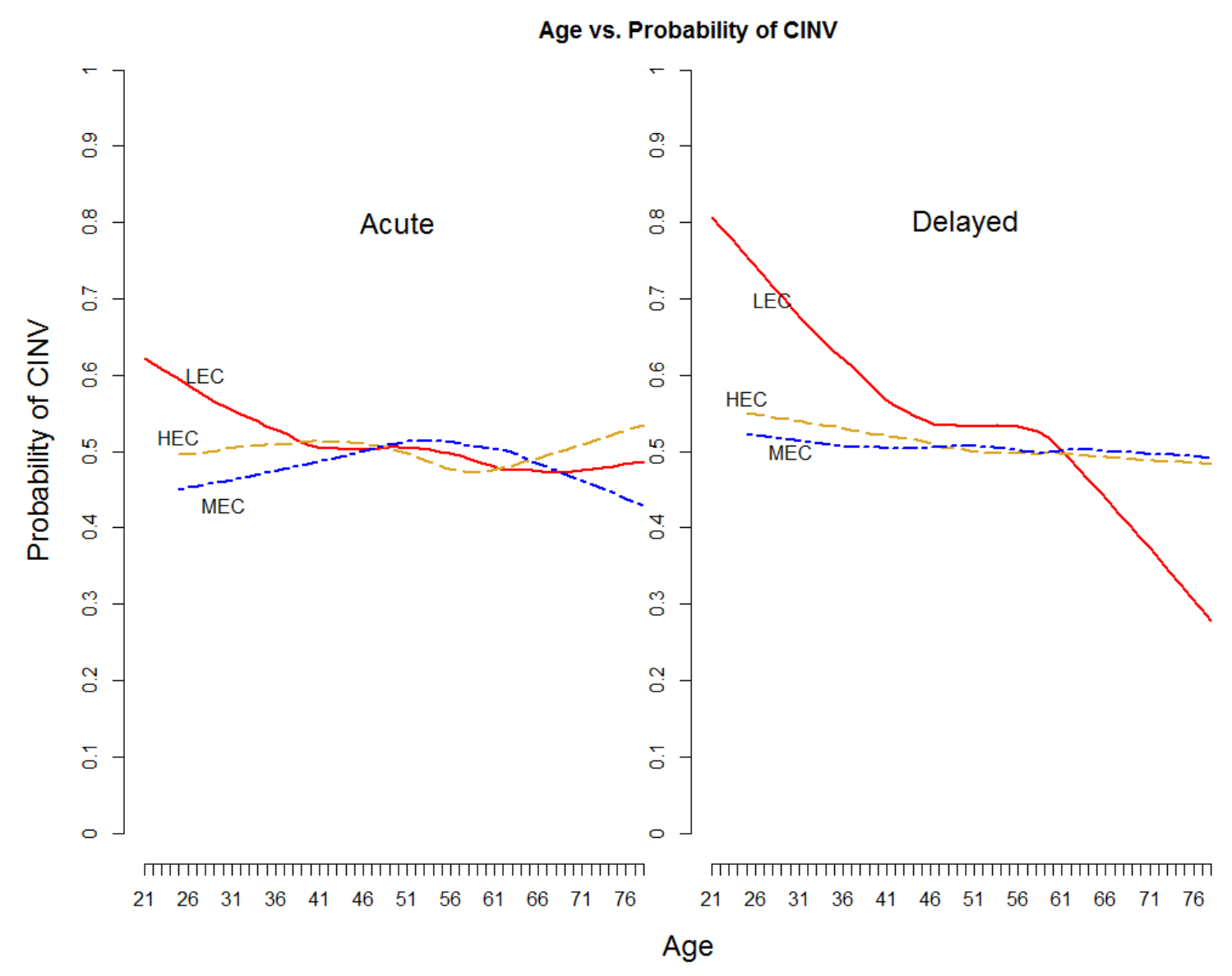

Figure 5: Age vs. Probability of CINV

\subsubsection{BMI vs. CINV Outcome}

In the literature, the BMI was found to be non-significant for CINV prediction. However, in our dataset, we observed that BMI has some impacts on the probability of CINV for LEC in both acute and delayed phase (Figure 6). In acute phase, the patients with lower BMI were at lower risk of CINV for low and moderate chemotherapies. In contrast, in delayed phase, patients with lower BMI were at higher risk of CINV for high emetogenic chemotherapies. However, in delayed phase, patients with higher BMI were at higher risk of CINV. 


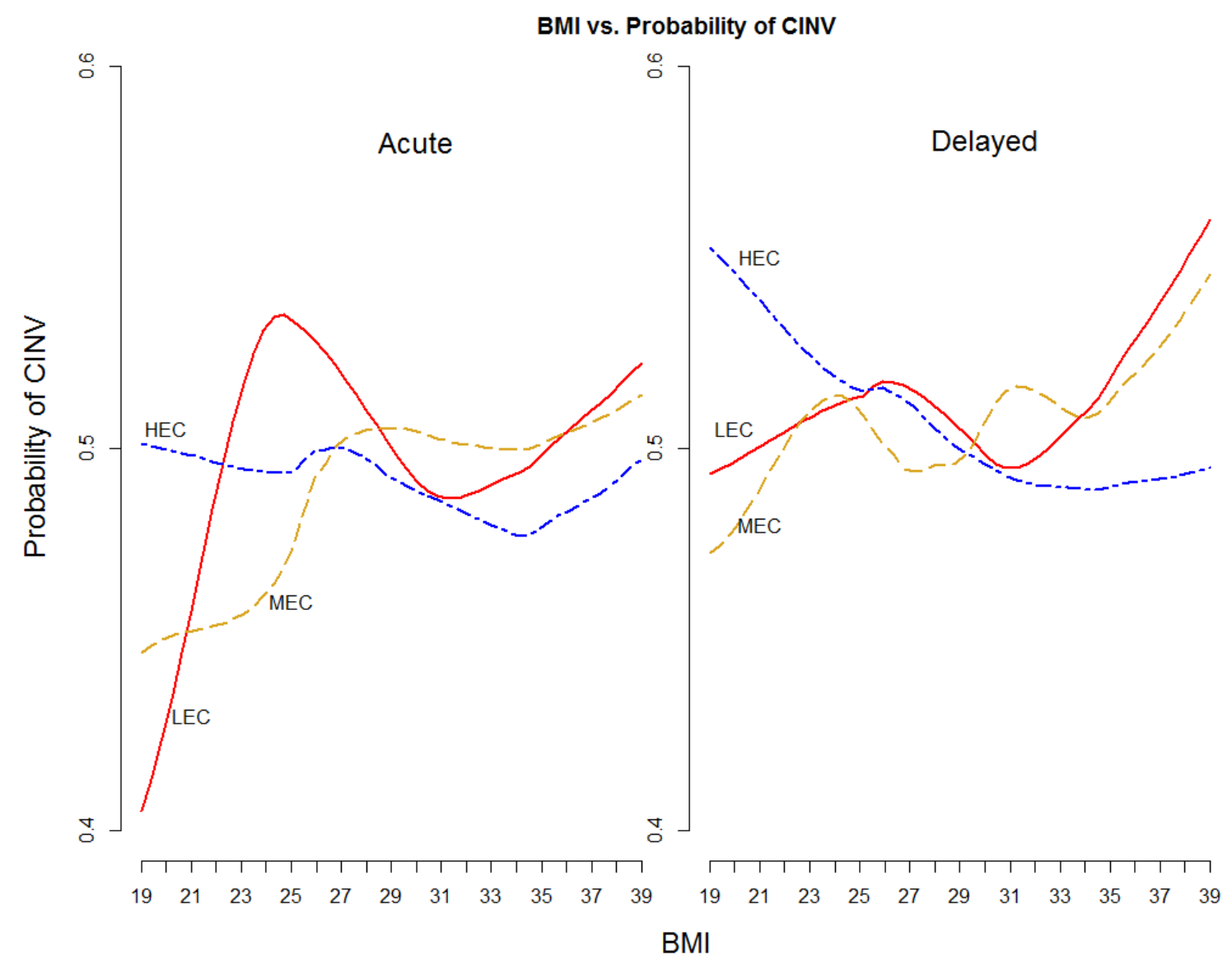

Figure 6: BMI vs. Probability of CINV

\subsubsection{Number of Comorbidities vs. CINV Outcome}

Figure 7 presents that the probability of CINV increases with the increase of number of comorbidities. For high emetogenic chemotherapies, the probability of CINV is about $73.21 \%$ for acute phase and $86.3 \%$ for delayed phase. For other cases, the probability is around $50 \%$. 
Number of Comorbidities vs. Probability of CINV
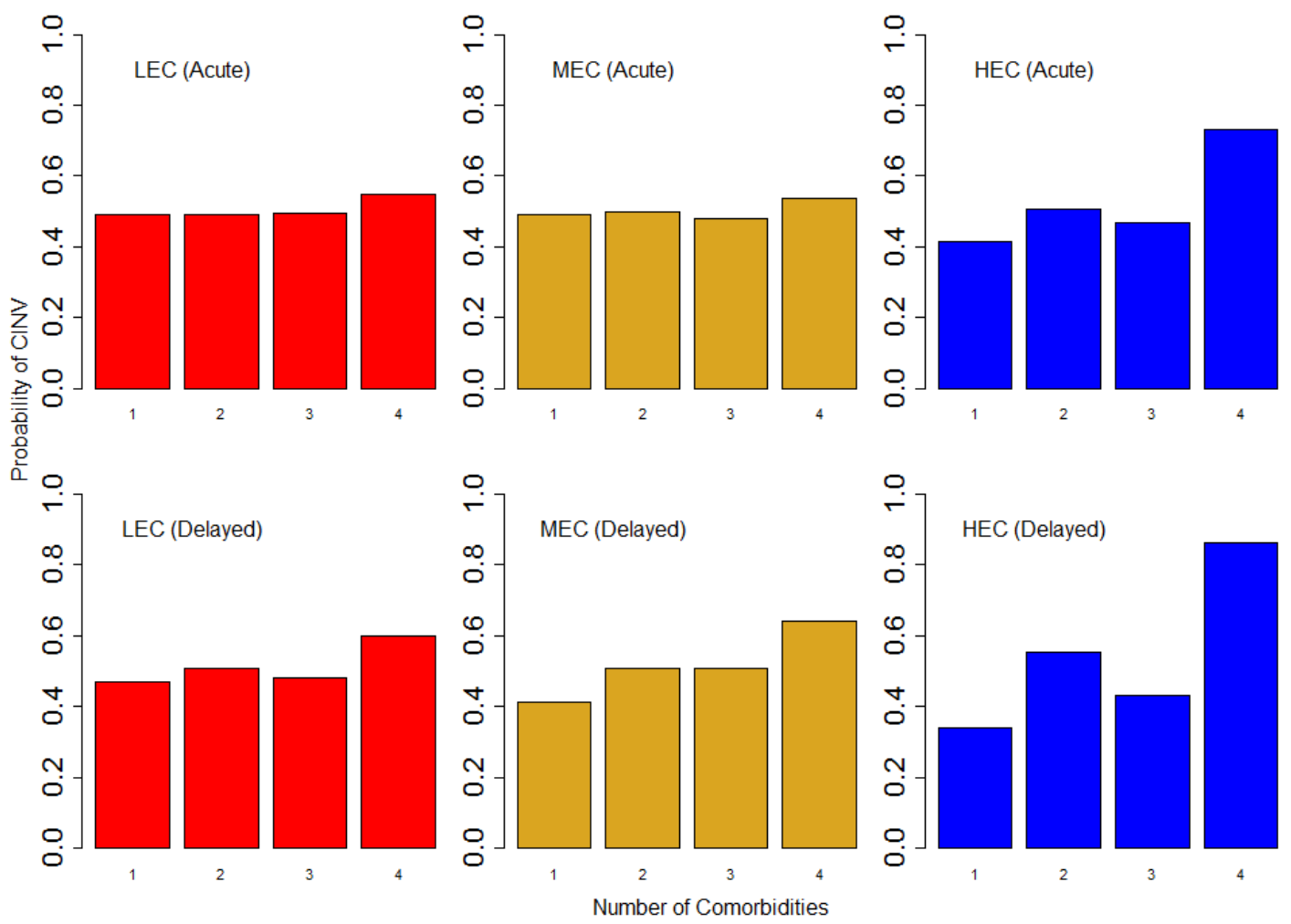

Figure 7: Number of Comorbidities vs. Probability of CINV

\subsubsection{Gender vs. CINV Outcome}

The probability of CINV for both males and females were around 50\% (Figure 8).

Overall this is not a good predictor but may have some impact in a sub population. 

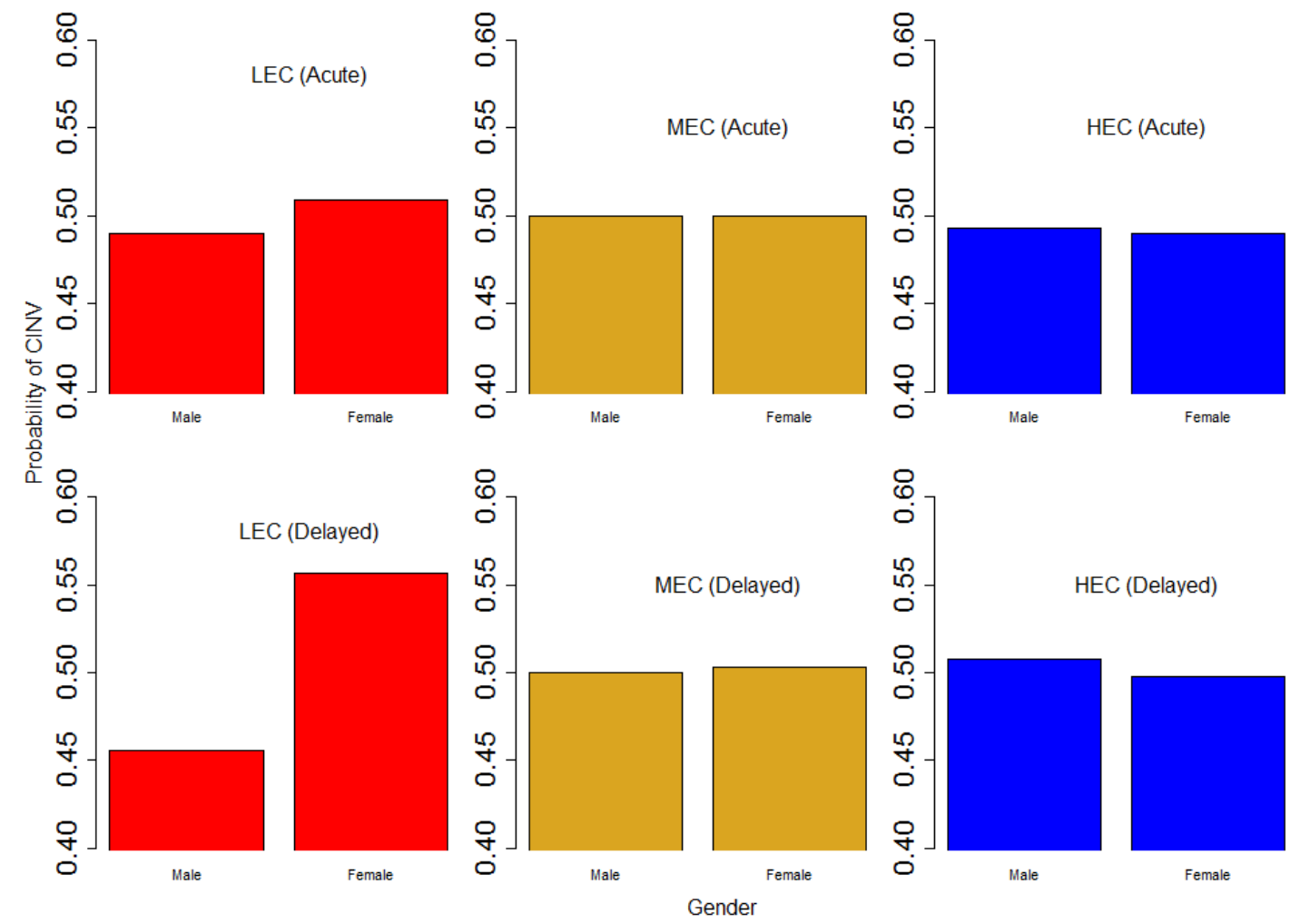

Figure 8: Gender vs. Probability of CINV

\subsubsection{Race vs. CINV Outcome}

In the dataset, the Hispanic population showed lower risk of CINV (around 40\% are at risk of CINV) than other population for both moderate and high emetogenic chemotherapies (Figure 9). Thus, race seems to have some predictive power for Hispanic population. Around 50\% of the White, African American and Asian population were at risk of CINV; thus, race does not seem to be a predictive factor for White, African American and Asian population. 
Race vs. Probability of CINV

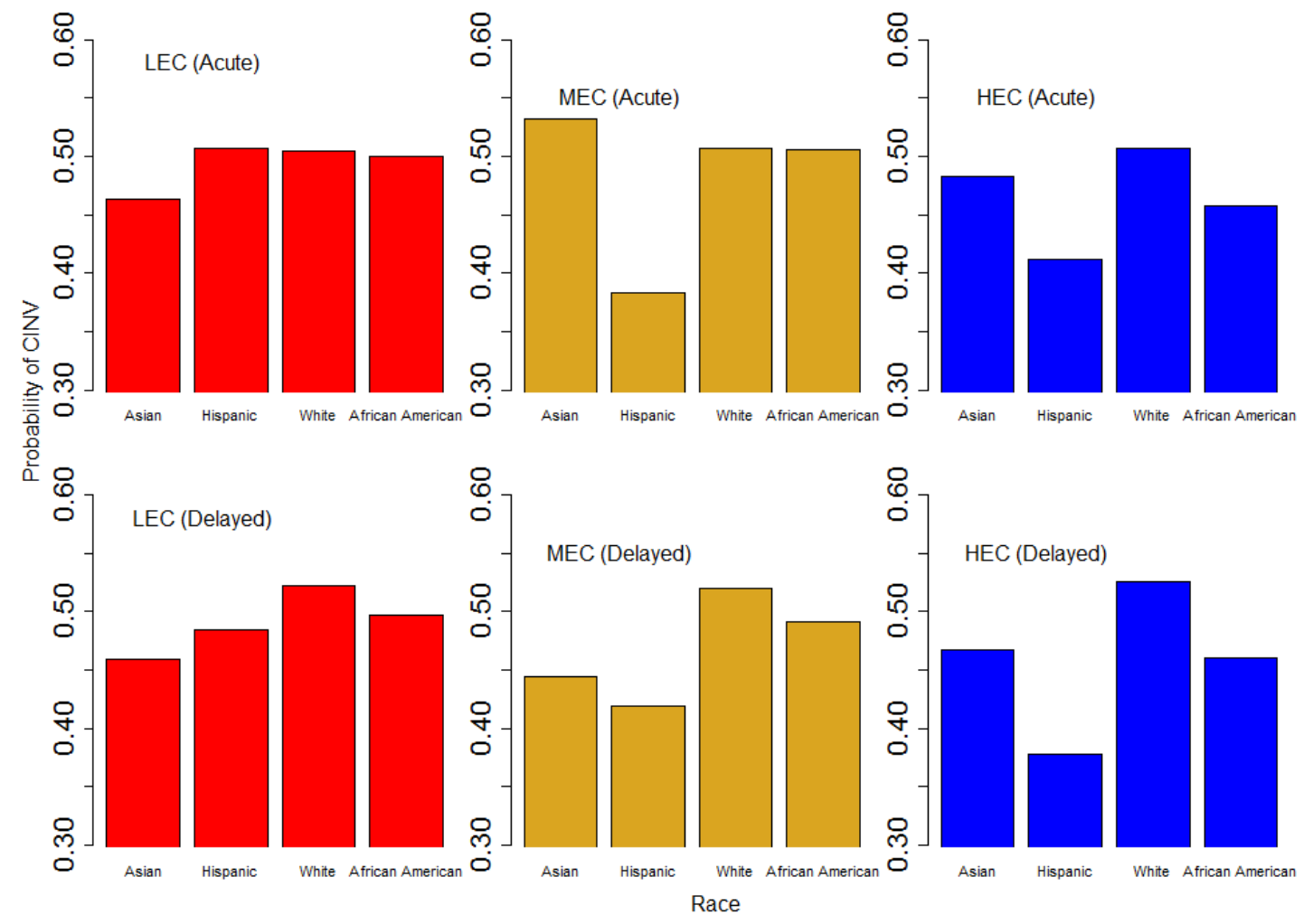

Figure 9: Race vs. Probability of CINV

\subsubsection{Stage of Cancer vs. CINV Outcome}

For high emetogenic chemotherapies, the patients are at similar risk of CINV with around $50 \%$ probability for any stages of cancer (Figure 10). However, stage 3 and 4 patients were at higher risk of CINV than stage 1 and 2 patients for low and moderate emetogenic chemotherapies in both phases. 
Stage of Cancer vs. Probability of CINV
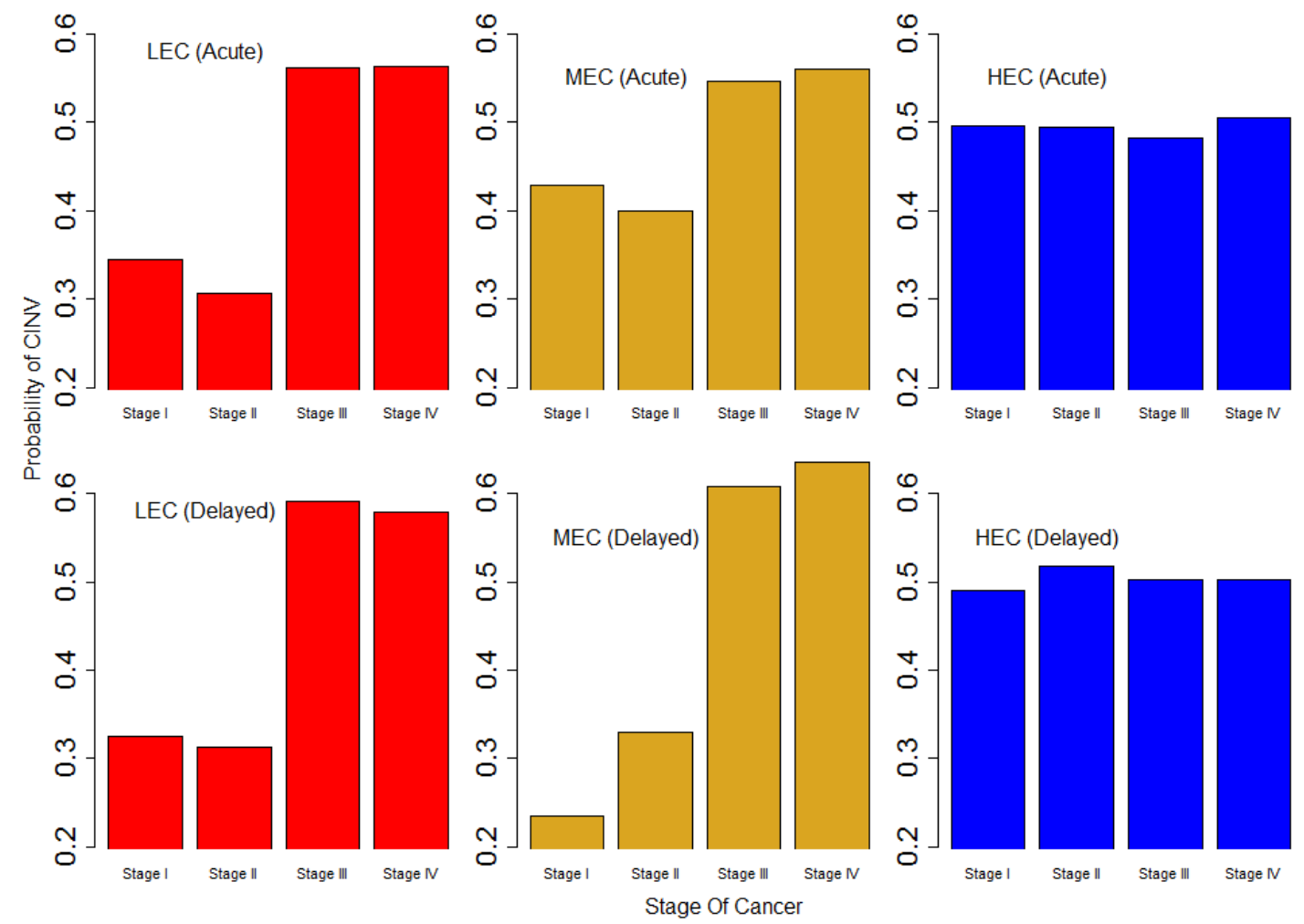

Figure 10: Stage of Cancer vs. Probability of CINV

\subsubsection{Alcohol Consumption vs. CINV Outcome}

The patients who drink lower amount of alcohol are at higher risk of CINV than the patients who drink higher amount of alcohol; similar pattern is observed for any type of chemotherapies (Figure 11). This is consistent with the findings in the literature. 
Alcohol Consumption vs. Probability of CINV
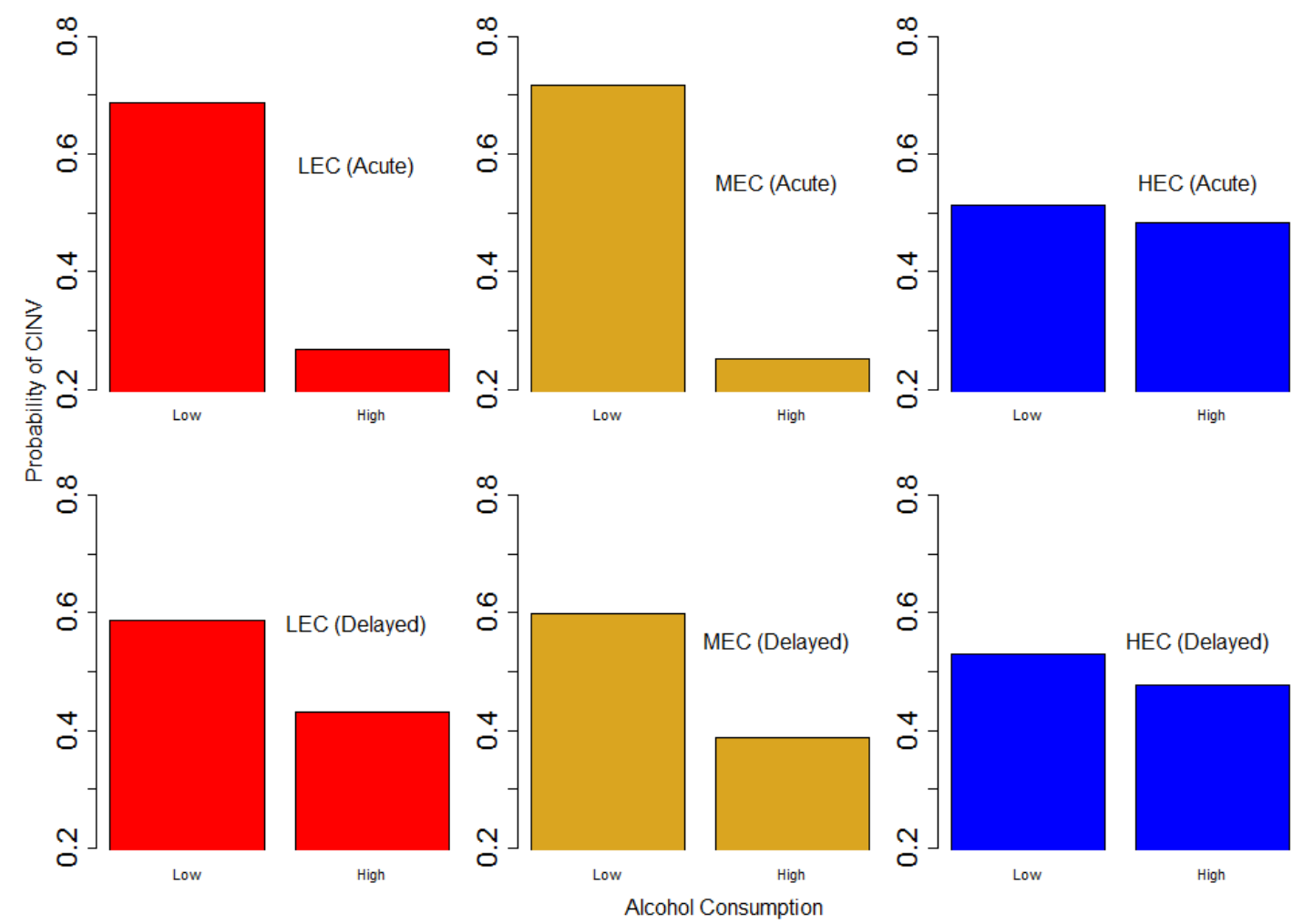

Figure 11: Alcohol Consumption vs. Probability of CINV

\subsubsection{Smoking vs. CINV Outcome}

The current smokers are at higher risk of CINV than the non-smokers. For smokers, the probability of CINV is higher at the acute phase than delayed phase (Figure 12). 


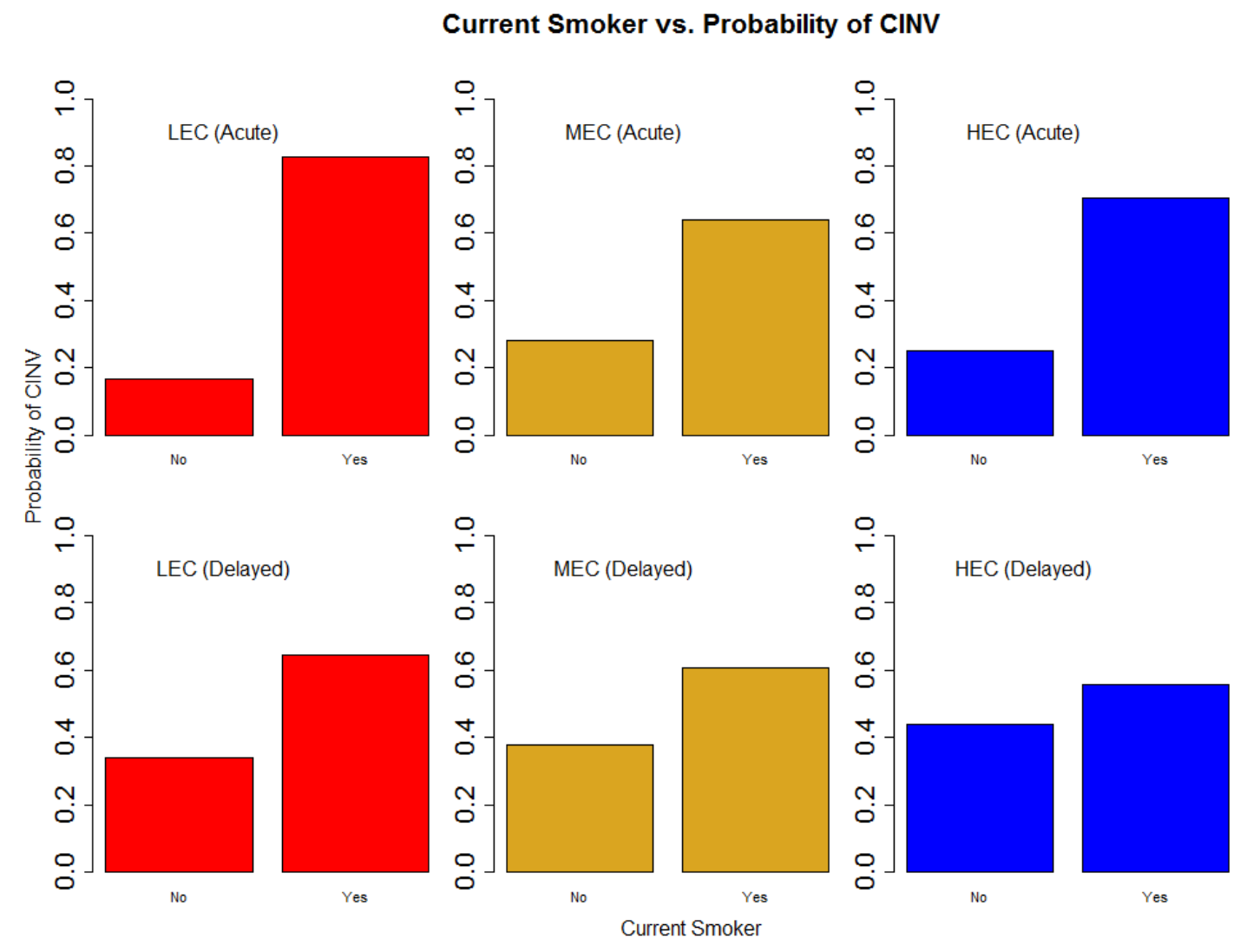

Figure 12: Current Smoker vs. Probability of CINV

\subsubsection{Dehydration vs. CINV Outcome}

The patients with dehydration symptom are at higher risk of CINV than the patients without having dehydration. In the CINV dataset, the probability of CINV varied from $73 \%$ to $83 \%$ depending on the context (Figure 13 ). 


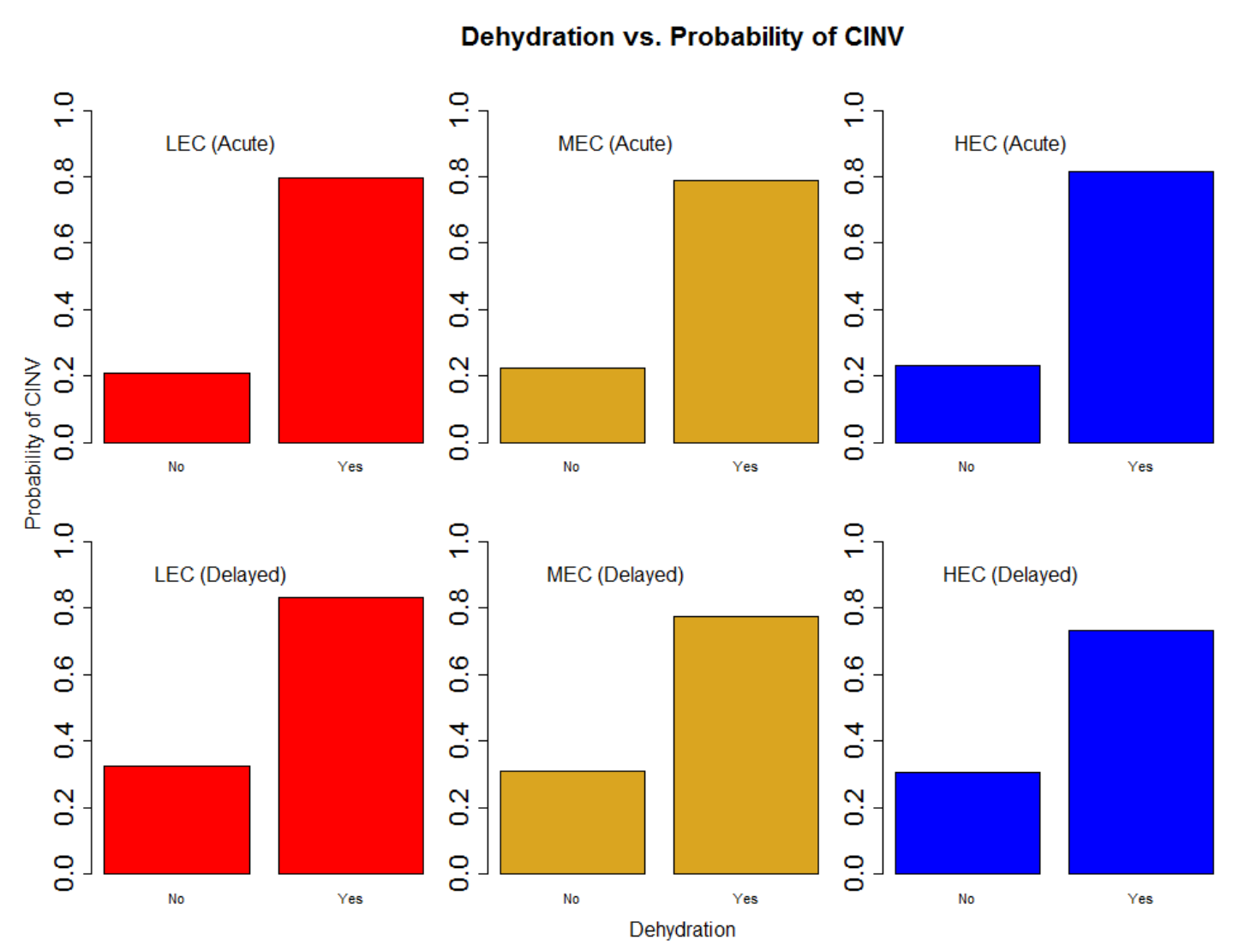

Figure 13: Dehydration vs. Probability of CINV

\subsubsection{Anxiety vs. CINV Outcome}

The patients having anxiety are at higher risk of CINV than the patients with no anxiety (Figure 14); the probability of CINV is higher at acute phase than delayed phase. 
Anxiety vs. Probability of CINV
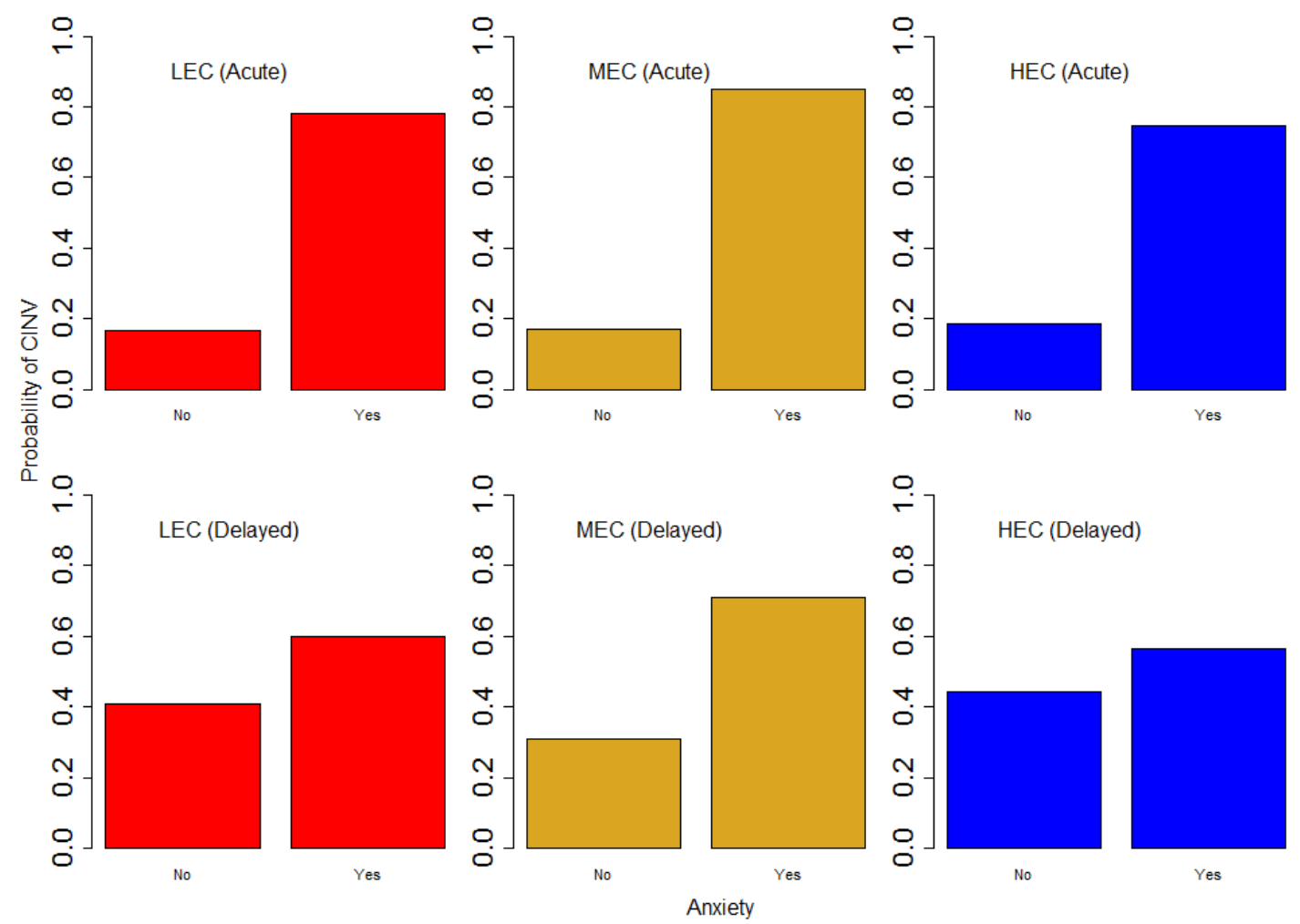

Figure 14: Anxiety vs. Probability of CINV

\subsubsection{Number of Previous Chemotherapy vs. CINV Outcome}

With the increase of number of previous chemotherapies, the probability of CINV has also increased. The patients who received more than 2 chemotherapy regimens were at very high risk of CINV (Figure 15). 
Number of Previous Chemotherapy vs. Probability of CINV
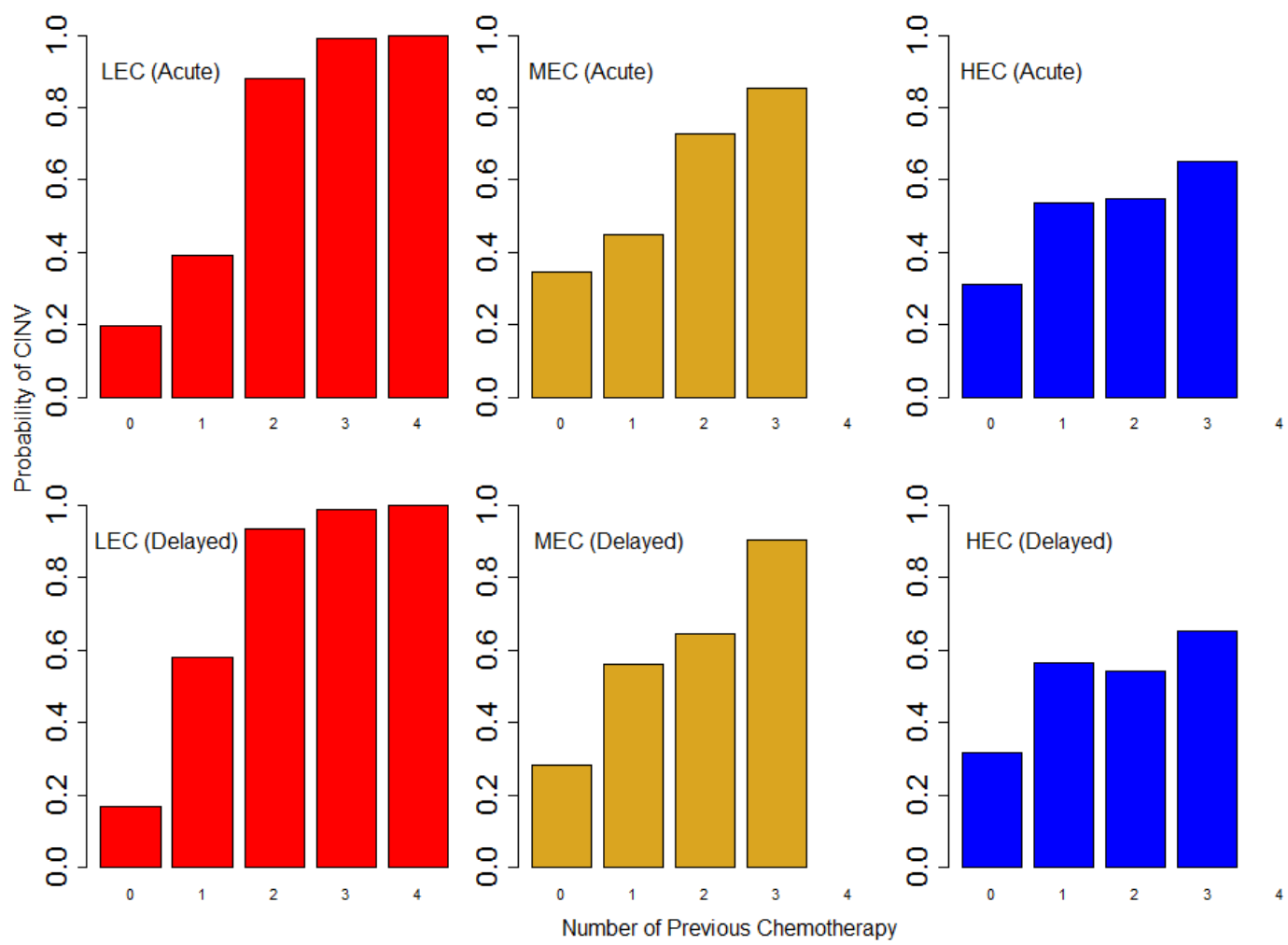

Figure 15: Number of Previous Chemotherapy vs. Probability of CINV

\subsubsection{Prior CINV vs. CINV Outcome}

The patients, who had CINV either in acute or delayed phase during the last cycle

of chemotherapy, were at higher risk of chemotherapy (Figure 16). The probability of CINV ranges from $72 \%$ to $86 \%$ percent in the acute phase and $57 \%$ to $82 \%$ in the delayed phase depending on the type of chemotherapy. 

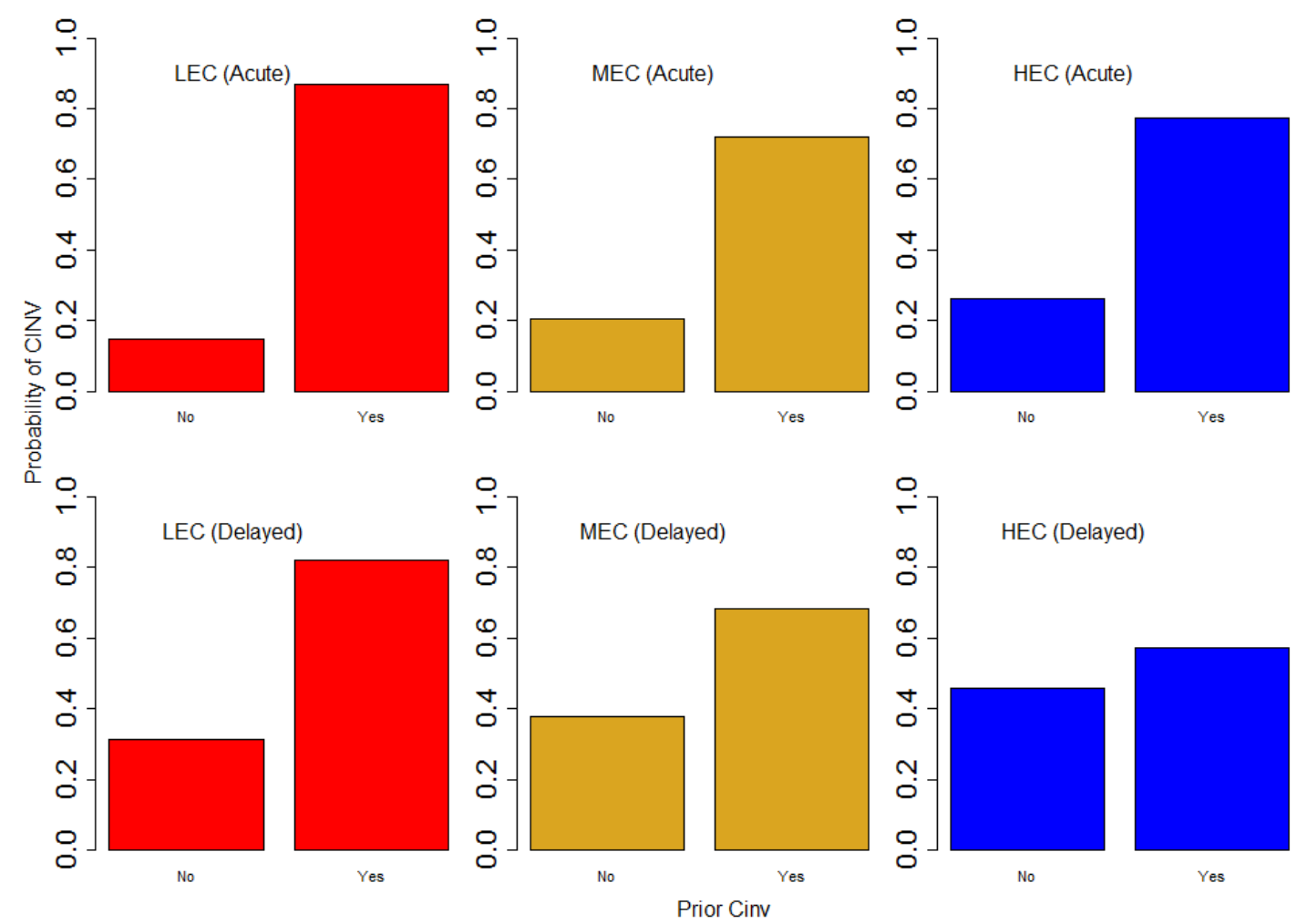

Figure 16: Prior CINV vs. Probability of CINV

\subsubsection{Type of Cancer vs. CINV Outcome}

The probability of CINV for various type of cancers varied based on the type of chemotherapy. In acute phase, leukemia and myeloma patients had higher probability of CINV for low emetogenic chemotherapies, colorectal cancer patients had higher probability of CINV for moderate emetogenic chemotherapies, and lung cancer and lymphoma patients had higher probability of CINV for high emetogenic chemotherapies. For delayed phase, lung cancer patients had higher probability of CINV for low emetogenic chemotherapies, colorectal cancer patients had higher probability of CINV for moderate 
emetogenic chemotherapies, and breast and lung cancer patients had higher probability of CINV for high emetogenic chemotherapies (Figure 17).

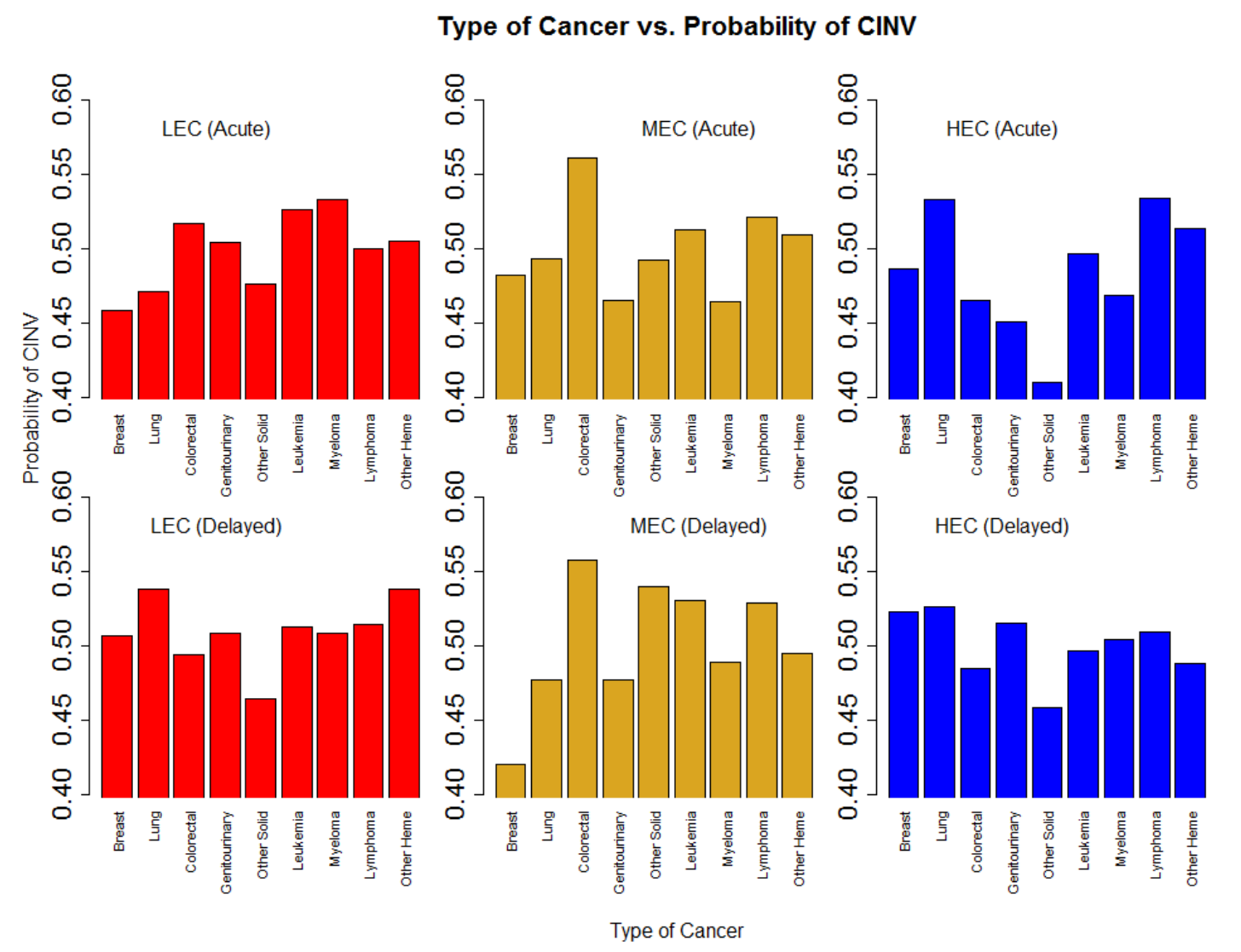

Figure 17: Type of Cancer vs. Probability of CINV 


\section{CHAPTER 4 : DATA MINING}

Data mining or knowledge discovery in databases (KDD) is a process of extracting previously unknown and potentially useful information or patterns from data. In general, data mining algorithms are categorized into two groups: descriptive or unsupervised learning and predictive or supervised learning. In supervised learning, the class labels of the observations or tuples are known whereas in unsupervised learning, the class labels of the tuples are unknown. Data mining techniques were found to be very useful in predictive analytics on medical data [104]. In this study, we needed to develop a prediction model, which falls into the category of supervised learning or classification.

\subsection{CLASSIFICATION METHODS}

Classification is a supervised learning method for building classification models based on a dataset (called training data) and the values in classifying attribute (called class label). The classification model is used to predict class label which is categorical. Classification is a two-step process in which the model is constructed in the first step and the accuracy of the model is determined using a dataset (called test dataset) in the second step. The accuracy of the classification model is the percentage of test dataset tuples that are correctly classified by the model. In order to overcome the over fitting problem, the test dataset must be independent of the training dataset. In general, the classification model 
consists of IF-THEN rules or mathematical formula. In the following sections, some classification techniques are described.

\subsubsection{Decision Tree}

The decision tree is a flow-chart-like tree structure in which the test on an attribute is presented by an internal or non-leaf node, the outcomes of the tests are presented by the branches, and the class labels correspond to the leaf nodes. The decision tree is constructed in a top-down, recursive, and divide-and-conquer manner. All attributes are assumed to be categorical; otherwise, continuous-valued attributes are discretized to form categories (e.g. age is a continuous-valued attribute which can be categorized into two groups: young and old). The key step in the decision tree construction process is the selection of attribute that best partitions the tuples into distinct classes. The attribute is selected based on the attribute selection measures such as information gain, gain ratio, or gini index, etc. At the beginning of the tree construction, all the training tuples are at the root node. The training tuples are recursively partitioned based on the selected attributes. The partitioning stops if the class label of all tuples in a given node is same, or no attributes left for partitioning, or no tuples left for partitioning.

In classification, the classification model corresponds to the decision tree from which the classification rules can easily be generated. In order to determine the unknown class label of a new observation, a path from root node to leaf node of the decision tree is traversed to test the attributes of the tuple against the corresponding non-leaf node and the class label of the tuple is determined by the leaf node.

The decision tree algorithms mainly differ based on the attribute selection measures and the tree pruning mechanisms. Iterative Dichotomiser 3 (ID3) was introduced by Ross 
Quinlan in 1979 [105], where information gain is used as the attribute selection measure. The information gain of an attribute is defined as the difference between the information required to classify a training tuple before and after the partitioning by the attribute. The attribute with the highest information gain is selected as the splitting criterion, which constructs a simple tree and minimizes the number of tests needed to classify a given tuple. The main drawback of the information gain measure is its biasness towards the attribute that corresponds to tests with many outcomes (i.e. branches).

In order to overcome the drawback of ID3, a successor of ID3 known as C4.5 was introduced by Quinlan [106]. C4.5 uses the gain ratio (an extension to information gain) as the attribute selection measure, which is defined as the ratio between the information gain and the split information value of the attribute. The split information value of an attribute is defined as the potential information generated by splitting the training dataset into a number of partitions, which corresponds to the outcomes of the test on the attribute. The attribute for splitting criterion is selected with the highest gain ratio. The main drawback of the gain ratio is that it becomes unstable when the split information value becomes zero. This scenario is handled by applying a constraint, which states that the information gain for a splitting criterion must be larger than all other splitting criterions. An improved version of C4.5 was developed by Quinlan, known as C5.0/See5 [107]. The C5.0 is a commercial data mining tool which is faster than C4.5 with lower error rate and smaller tree size, and memory requirement for large dataset is also less.

The Classification and Regression Trees (CART) [108] were invented by Brieman et al. in parallel to the ID3 around the same time, which is a generation of binary decision tree. The Gini index is used as the attribute selection measure in CART. The Gini index 
measures the impurity of the training dataset $\mathrm{D}$, which is defined as: $\operatorname{Gini}(D)=1-$ $\sum_{i=1}^{m} p_{i}^{2}$, where $p_{i}^{2}$ is the probability that a tuple in D belongs to class $\mathrm{Ci}$. The Gini split for splitting D into D1 and D2 of size $\mathrm{n} 1$ and $\mathrm{n} 2$ respectively, based on attribute $\mathrm{A}$, is defined as: $\operatorname{Gini}_{A}(D)=\frac{n_{1}}{n} \operatorname{Gini}\left(D_{1}\right)+\frac{n_{2}}{n} \operatorname{Gini}\left(D_{2}\right)$. The reduction in impurity is measured by the difference between Gini(D) and GiniA(D). The attribute with the maximum reduction in impurity (or minimum Gini index) is selected as the splitting criteria.

After tree construction, the branches, that reflect anomalies in the training data due to noise or outliers, are identified and removed (known as tree pruning step) to overcome the over-fitting of the data and to improve the classification accuracy. Two approaches are adopted for tree pruning: pre-pruning and post-pruning. In pre-pruning, the tree construction is halted early depending on the predefined threshold for splitting criterion of a node and the node becomes a leaf. The post-pruning approach is more common, in which sub-trees are removed from the constructed tree at a node and the node becomes a leaf. In CART, the pruning algorithm is known as "cost complexity" (a post-pruning approach), which is a function of number of leaves and error rate. The tree with smallest size and minimum cost complexity is chosen. In $\mathrm{C} 4.5$, the pruning algorithm is known as "pessimistic pruning", which is similar to cost complexity. The main difference between these two algorithms is that, the cost complexity algorithm uses the pruning dataset (independent of training dataset) and the pessimistic pruning algorithm uses the training dataset for error rate estimation. Another algorithm for tree pruning is based on the Minimum Description Length (MDL) principle. A hybrid approach combining pre-pruning and post-pruning can also be applied. 


\subsubsection{Naïve Bayesian Classification}

The Naïve Bayesian classification is the simplification of Bayesian classification [109]. The Bayesian classifier predicts the probability of class membership of a given tuple and is based on the Bayes' theorem. The Bayes' theorem requires initial knowledge of many probabilities, that is, computational cost is significant. The Naïve Bayesian classifier simplifies the computation involved by applying an assumption called class conditional independence; that is, values of different attributes in a given class are independent of each other. However, in many cases class conditional assumption is unrealistic, particularly in medical data, where many attributes containing patient symptoms and health conditions are strongly related (for example, blood pressure and body mass index). As such, this assumption is a main drawback of Naïve Bayesian classifier. In general, the performance of Naïve Bayesian classifier is better than decision trees and also demonstrates high speed and accuracy on large datasets. As a result, the Naïve Bayesian classifier is widely used in medical data mining regardless of violations of attribute independence assumption.

\subsubsection{Neural Network}

The neural network is a backpropagation learning algorithm, which is a set of weighted connection between input/output units. During construction of the classification model, the weights of the connections are adjusted to correctly predict the class label of the input tuple [110]. The main drawbacks of the neural network are longer training time and poor interpretability. However, the computation time of the neural network can be reduced by parallel computation as it is inherently parallel in nature. The advantages of neural network include high tolerance of noisy data, ability to classify patterns, and suitability for 
continuous-valued inputs and outputs. It is also useful for classifying a dataset without or little prior knowledge of relationships between attributes and classes. This classification technique is widely used in pathology and laboratory medicine.

\subsection{DATA MINING TOOLS}

There are several tools available for data mining. We used two most widely used tools called WEKA[111] and R [112].

\subsection{MODEL VALIDATION}

Three performance measures were compared for benchmarking: accuracy, sensitivity, and specificity. 10-fold cross validation were used for model validations. The dataset is divided into 10 parts. In each iteration, 9 parts were used for model construction and one part was used for model validation.

\subsection{DATA MINING RESULTS}

In this section, the data mining results for six individual datasets are presented. We primarily mined the datasets using the decision tree algorithm in WEKA (called J48) [106] and compared the prediction results with four other algorithms: (1) Naïve Bayes [113], (2) Logistic Regression classifier[114], (3) Neural Network (Voted Perceptron) [115], and (4) Support Vector Machine (using sequential minimal optimization) [116]. The rationale behind using decision tree as the primary model was two-fold. First, the model is easy to explain to the clinicians, and (2) the model usually provides better prediction performance. 


\subsubsection{Decision Tree (Dataset: Acute, LEC)}

Figure 18 presents the decision tree model for predicting CINV in the acute phase for low emetogenic chemotherapies. The model predicts $94.8 \%$ of the records correctly with $96.3 \%$ correct predictions for the positive outcome of CINV (i.e., sensitivity) and 93.3\% correct predictions for the negative outcome of CINV (i.e., specificity). The performance of the model was compared with Neural Network, Logistic Regression, Support Vector Machine, and Naïve Bayes (Figure 19). Overall, the best performance was given by Naïve Bayes; the accuracy is $96.6 \%$, the sensitivity is $96.3 \%$, and the specificity is $96.8 \%)$. 


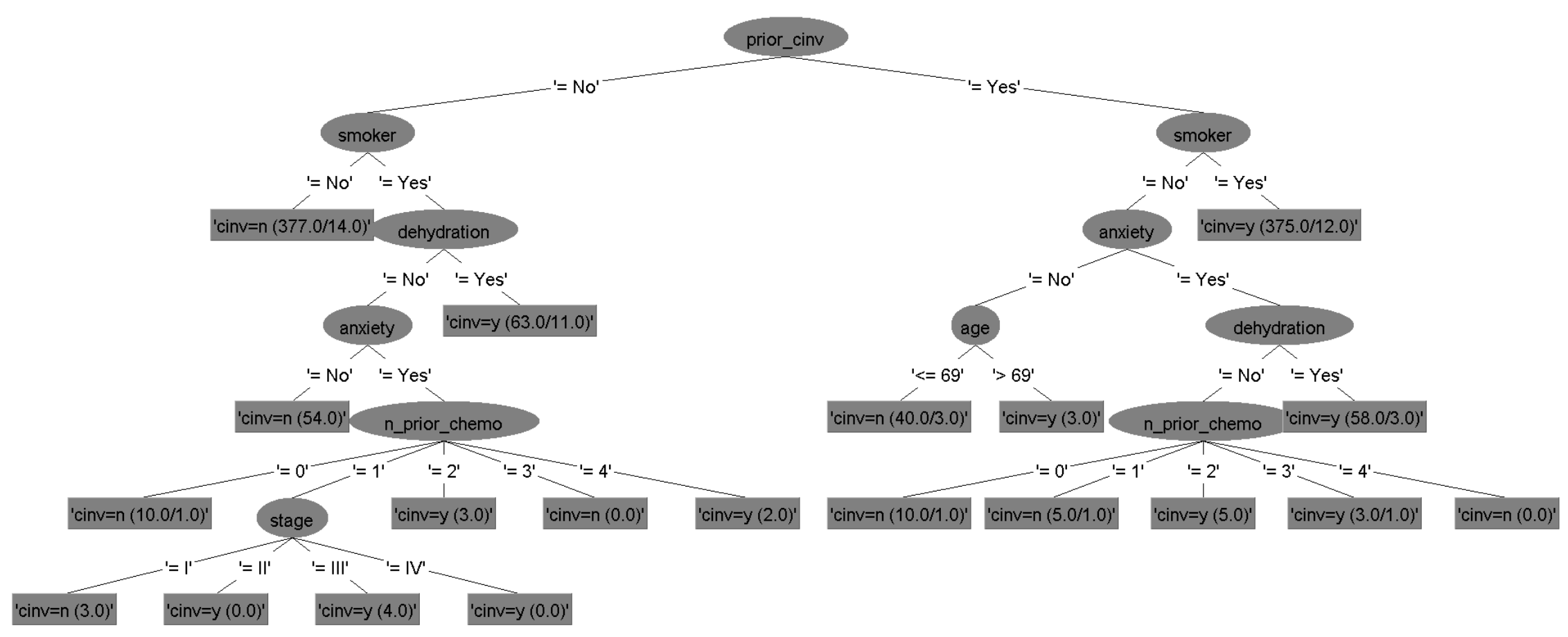

Figure 18: Decision Tree - Acute, LEC 


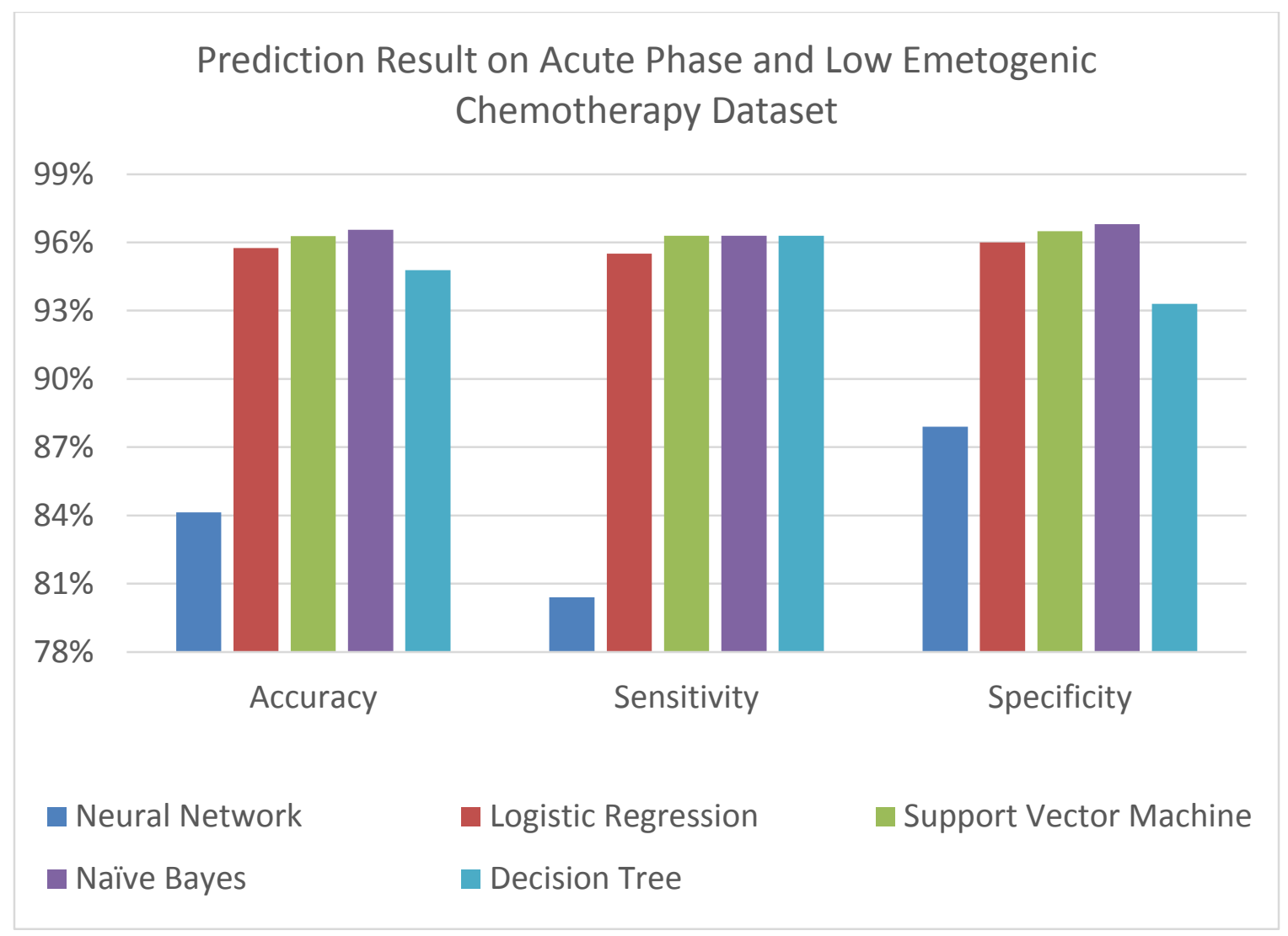

Figure 19: Prediction Result on Acute Phase and Low Emetogenic Chemotherapy Dataset

\subsubsection{Decision Tree (Dataset: Delayed, LEC)}

Figure 20 presents the decision tree model for predicting CINV in the delayed phase for low emetogenic chemotherapies. The model predicts $88.5 \%$ of the records correctly with $88.2 \%$ correct predictions for the positive outcome of CINV and $88.7 \%$ correct predictions for the negative outcome of CINV. The performance of the model was compared with Neural Network, Logistic Regression, Support Vector Machine, and Naïve Bayes (Figure 21). Overall, the best performance is given by Support Vector Machine; the accuracy is $89.5 \%$, the sensitivity is $87.8 \%$, and the specificity is $91.3 \%$. 


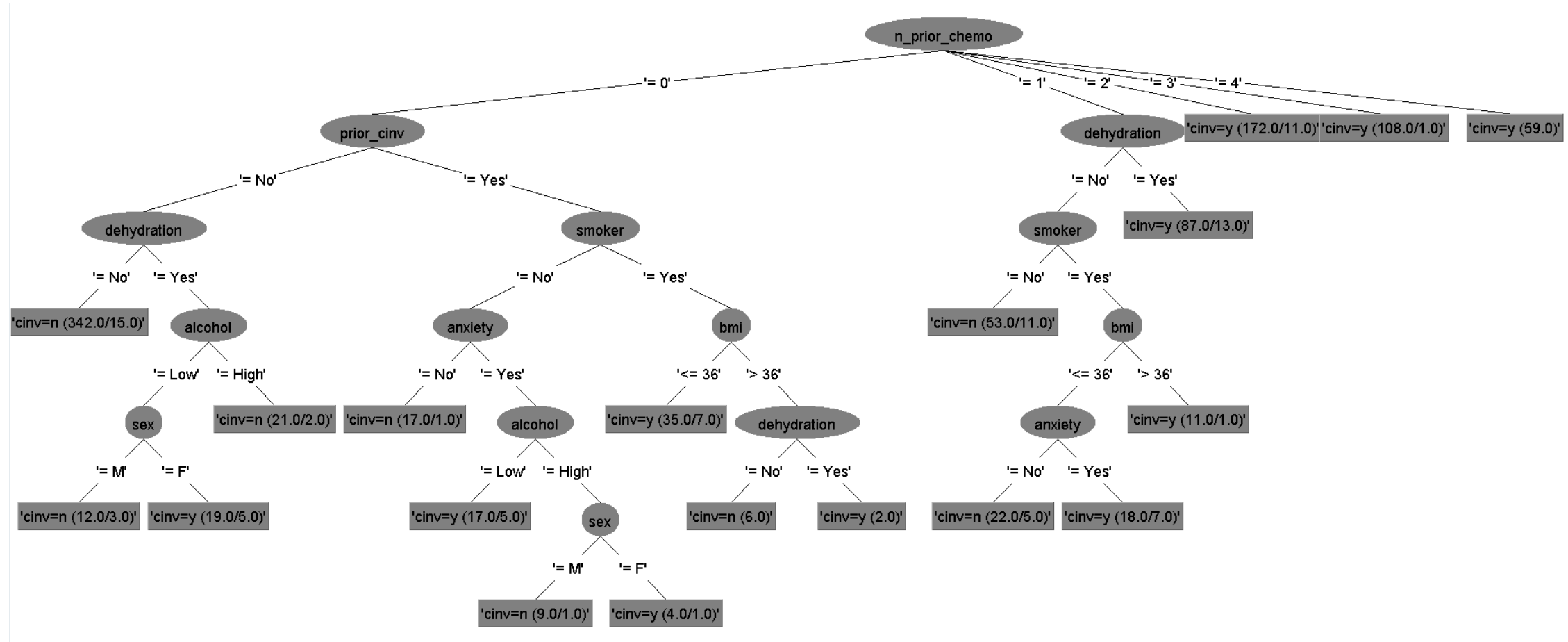

Figure 20: Decision Tree - Delayed, LEC 


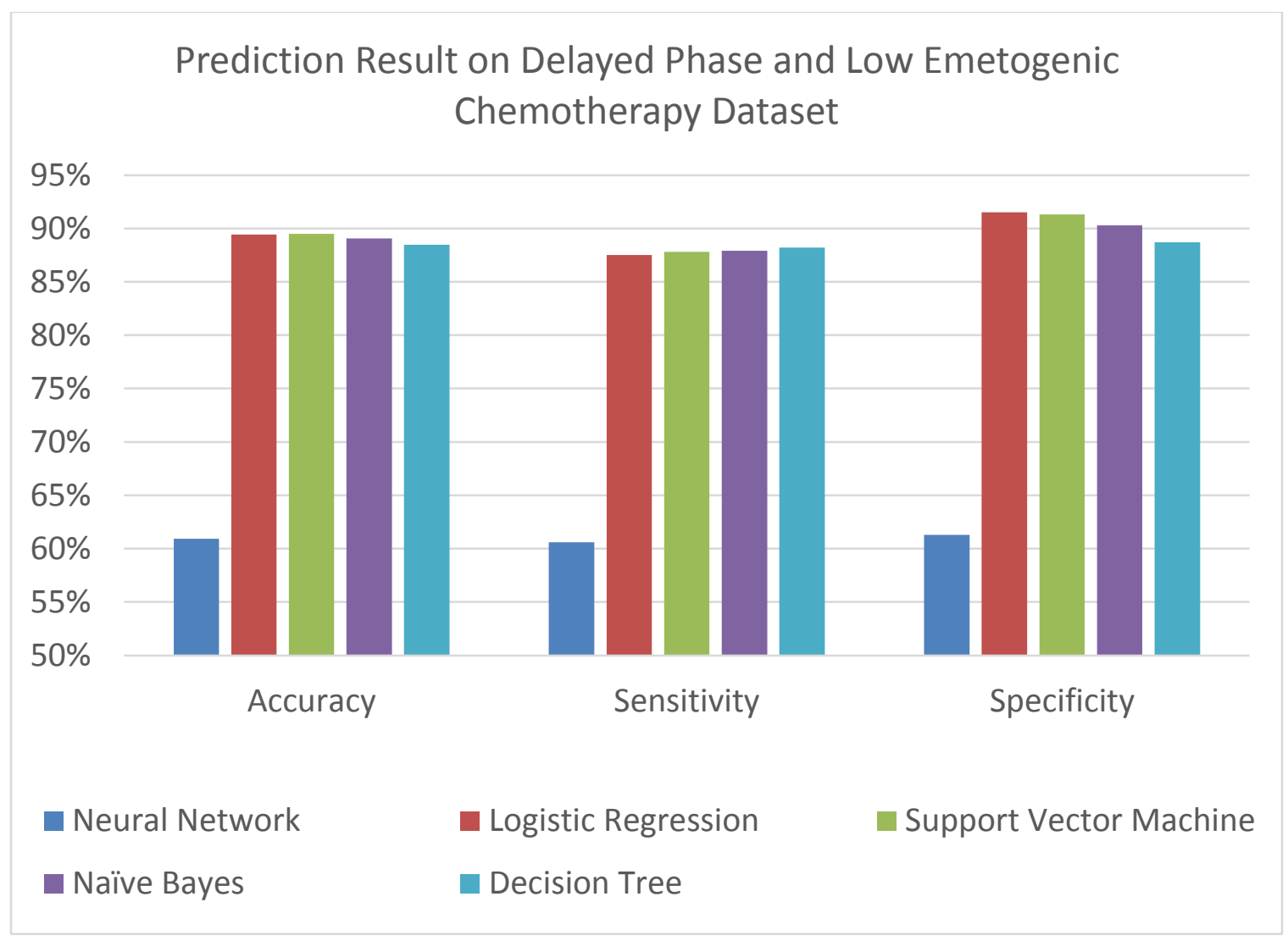

Figure 21: Prediction Result on Delayed Phase and Low Emetogenic Chemotherapy Dataset

\subsubsection{Decision Tree (Dataset: Acute, MEC)}

Figure 22 presents the decision tree model for predicting CINV in the acute phase for moderate emetogenic chemotherapies. The model predicts $90.2 \%$ of the records correctly with $90.3 \%$ correct predictions for the positive outcome of CINV and $90.1 \%$ correct predictions for the negative outcome of CINV. The performance of the model was compared with Neural Network, Logistic Regression, Support Vector Machine, and Naïve Bayes (Figure 23Figure 19). Overall, the best performance is given by Naïve Bayes; the accuracy is $90.8 \%$, the sensitivity is $89.3 \%$, and the specificity is $92.3 \%$. 


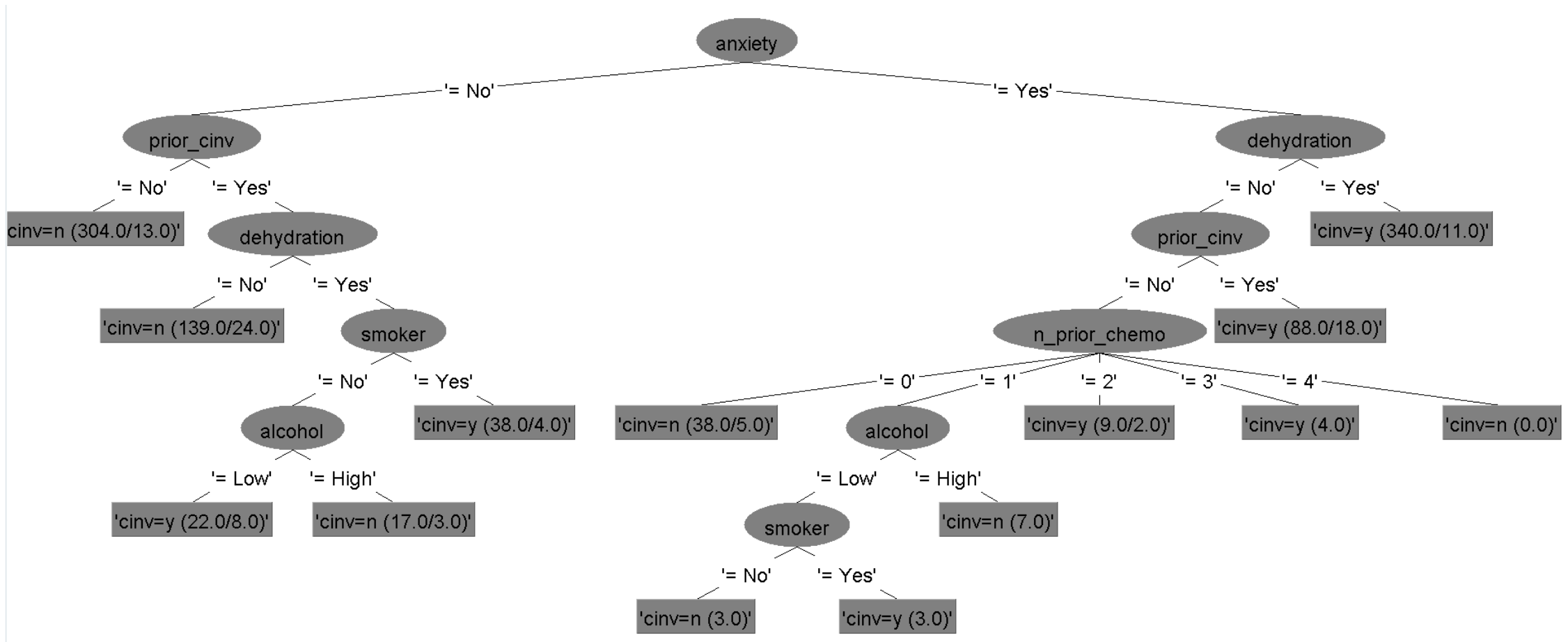

Figure 22: Decision Tree - Acute, MEC 


\section{Prediction Result on Acute Phase and Moderate Emetogenic Chemotherapy Dataset}

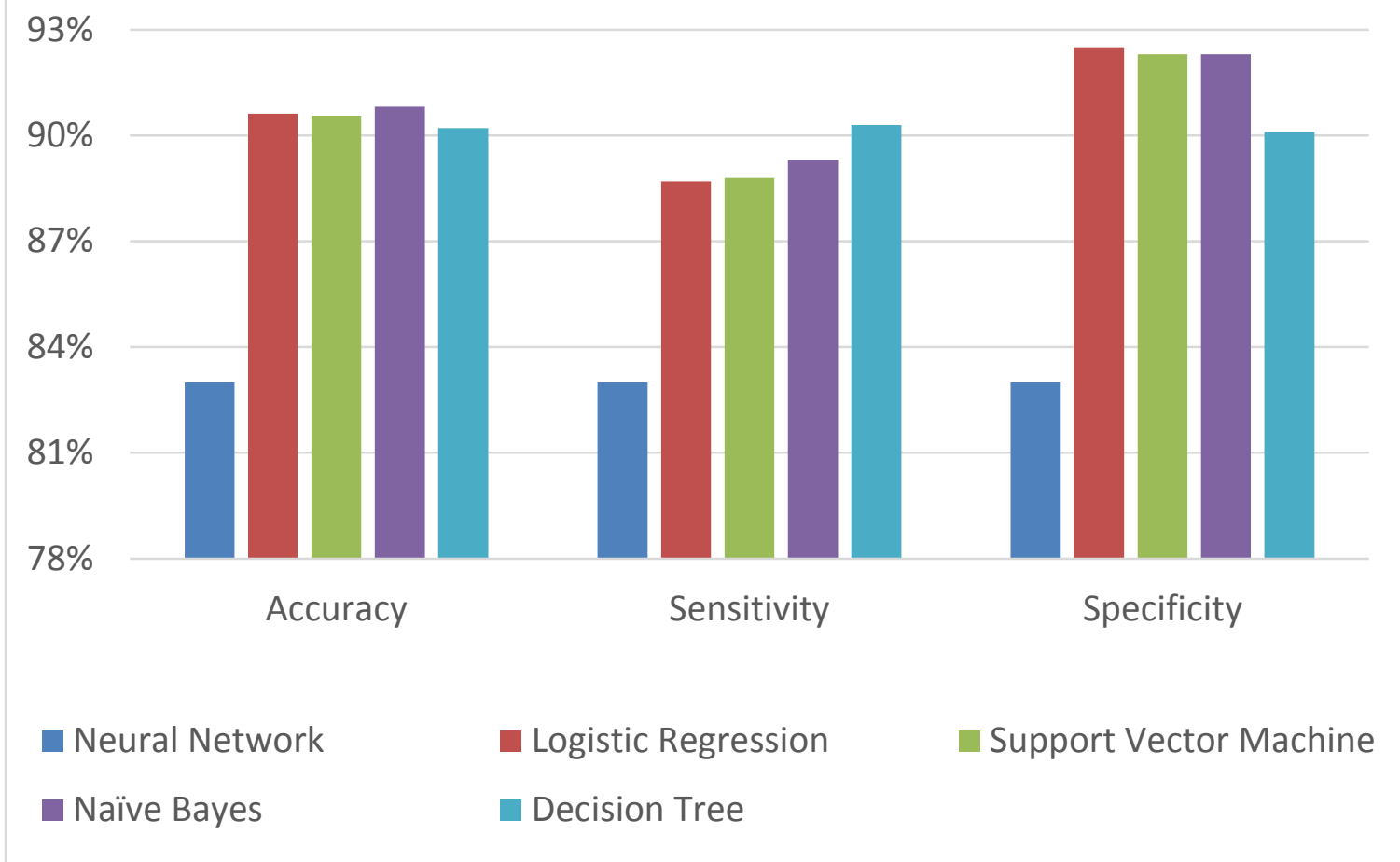

Figure 23: Prediction Result on Acute Phase and Moderate Emetogenic Chemotherapy Dataset

\subsubsection{Decision Tree (Dataset: Delayed, MEC)}

Figure 24 presents the decision tree model for predicting CINV in the delayed phase for moderate emetogenic chemotherapies. The model predicts $79.3 \%$ of the records correctly with $76.3 \%$ correct predictions for the positive outcome of CINV and $82.4 \%$ correct predictions for the negative outcome of CINV. The performance of the model was compared with Neural Network, Logistic Regression, Support Vector Machine, and Naïve Bayes (Figure 25Figure 19). Overall, the best performance is given by Naïve Bayes; the accuracy is $81.5 \%$, the sensitivity is $81.7 \%$, and the specificity is $81.3 \%$. 


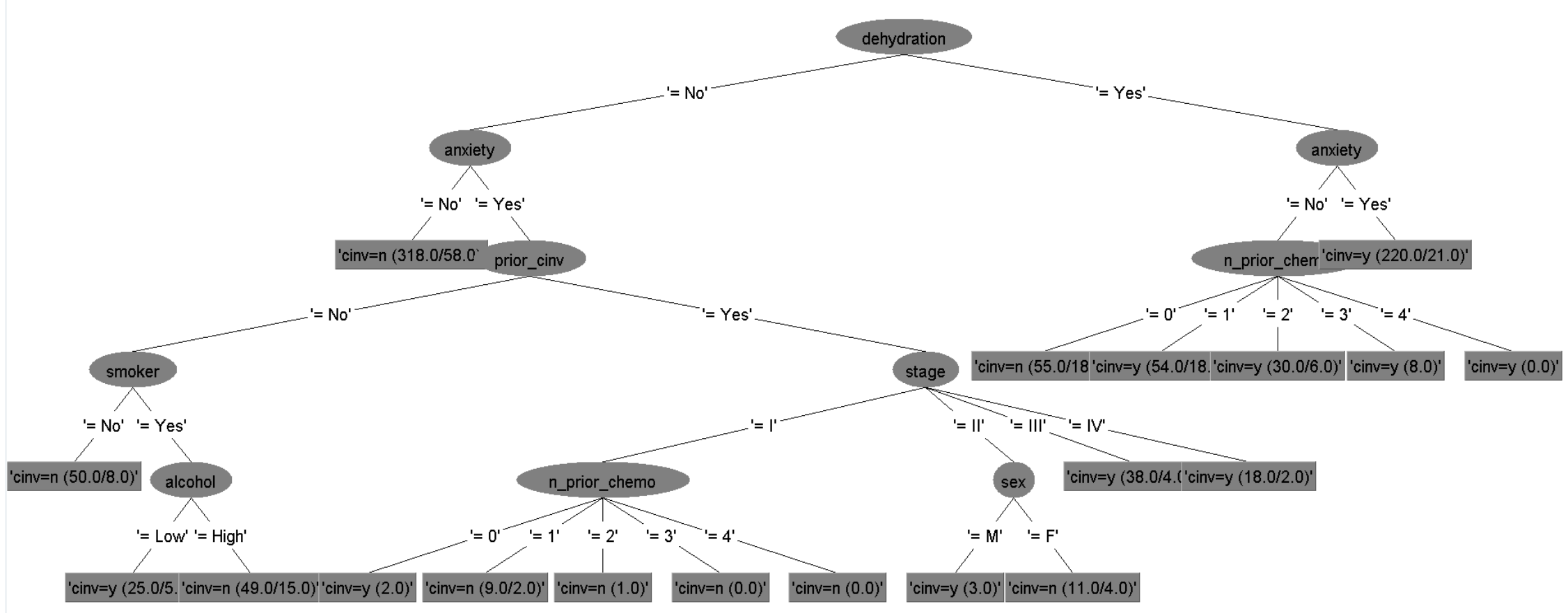

Figure 24: Decision Tree - Delayed, MEC 


\section{Prediction Result on Delayed Phase and Moderate Emetogenic Chemotherapy Dataset}

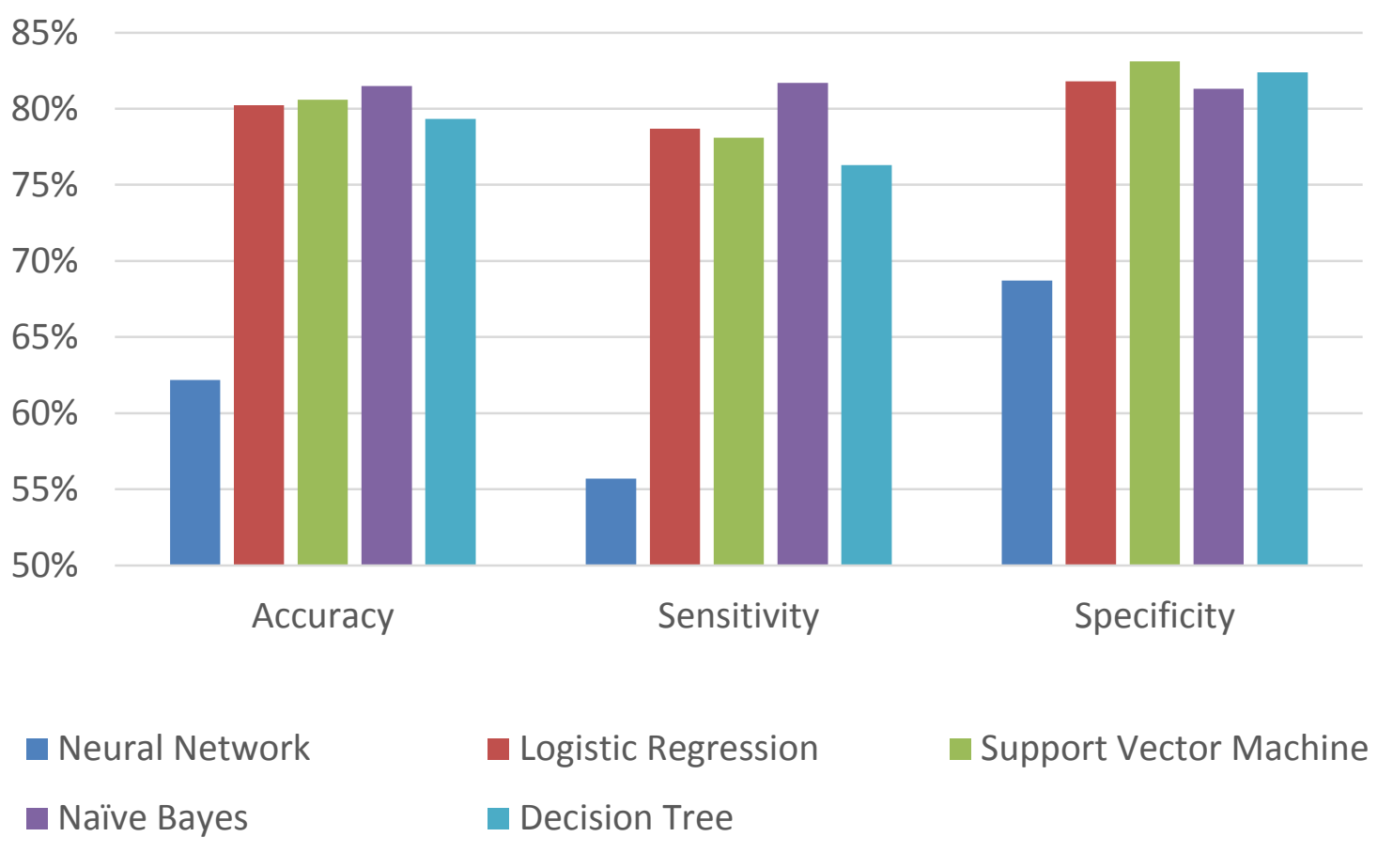

Figure 25: Prediction Result on Delayed Phase and Moderate Emetogenic Chemotherapy Dataset

\subsubsection{Decision Tree (Dataset: Acute, HEC)}

Figure 26 presents the decision tree model for predicting CINV in the acute phase for high emetogenic chemotherapies. The model predicts $88.7 \%$ of the records correctly with $90.3 \%$ correct predictions for the positive outcome of CINV and 87.2\% correct predictions for the negative outcome of CINV. The performance of the model was compared with Neural Network, Logistic Regression, Support Vector Machine, and Naïve Bayes (Figure 27Figure 19). Overall, the best performance is given by the Decision Tree model. The second best performance is given by Naïve Bayes; the accuracy is $88.6 \%$, the sensitivity is $88.5 \%$, and the specificity is $88.7 \%$. 


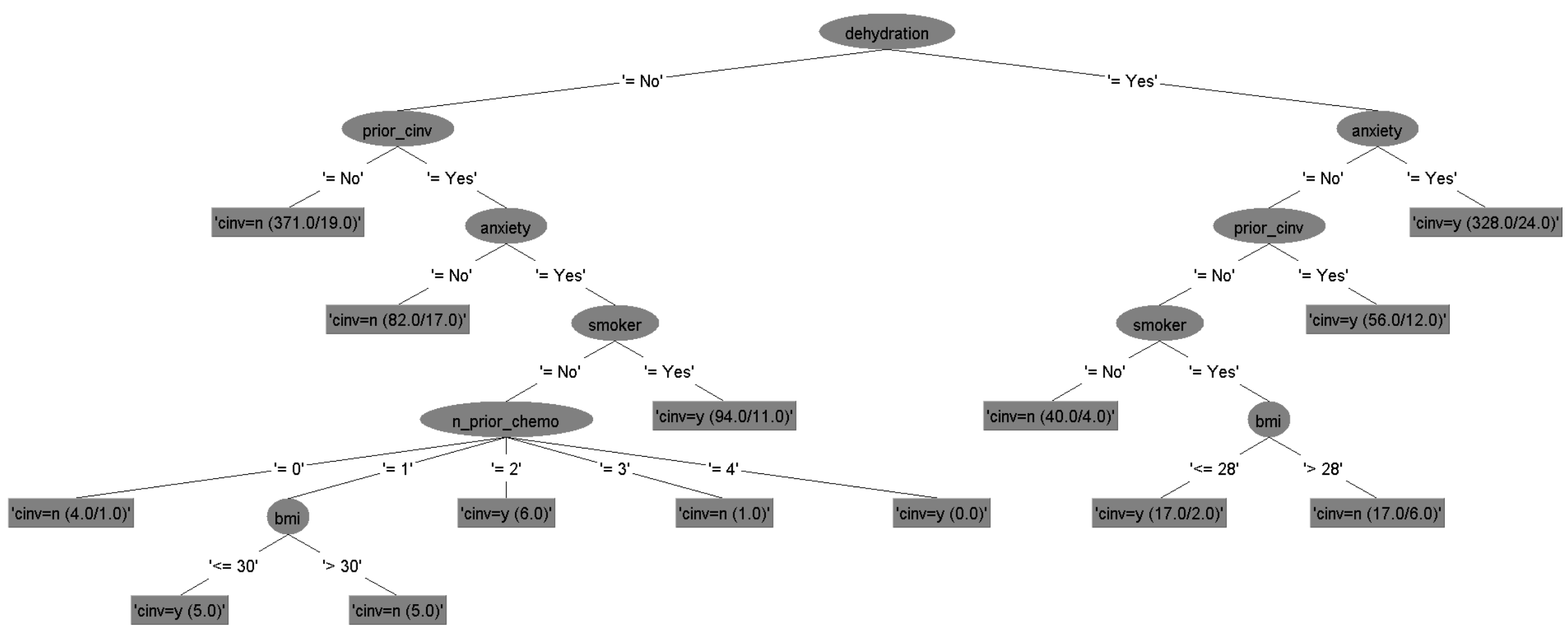

Figure 26: Decision Tree - Acute, HEC 


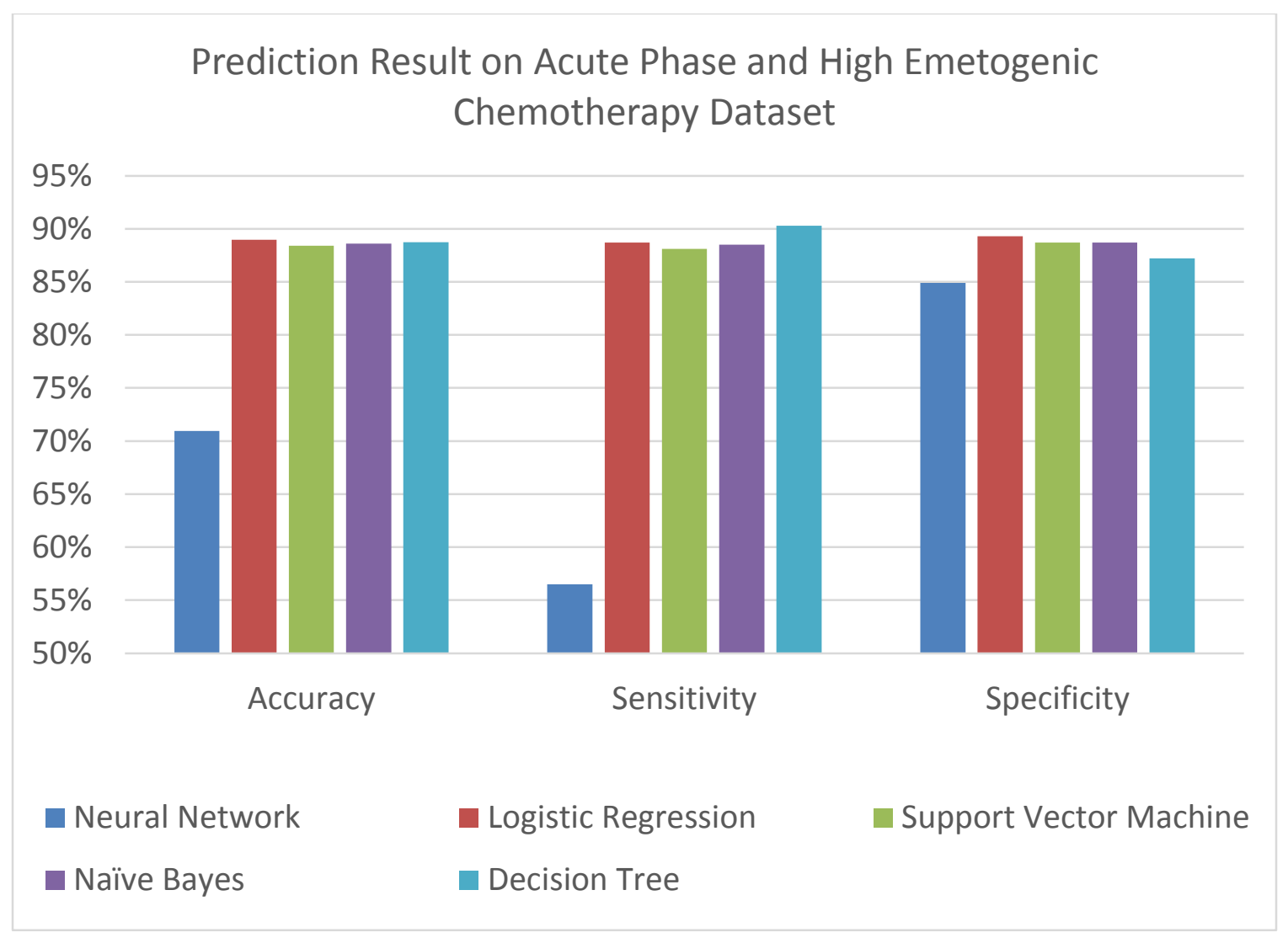

Figure 27: Prediction Result on Acute Phase and High Emetogenic Chemotherapy Dataset

\subsubsection{Decision Tree (Dataset: Delayed, HEC)}

Figure 28 presents the decision tree model for predicting CINV in the delayed phase for high emetogenic chemotherapies. The model predicts $81.0 \%$ of the records correctly with $85.2 \%$ correct predictions for the positive outcome of $\mathrm{CINV}$ and $76.7 \%$ correct predictions for the negative outcome of CINV. The performance of the model was compared with Neural Network, Logistic Regression, Support Vector Machine, and Naïve Bayes (Figure 29Figure 19). Overall, the best performance is given by the Decision Tree model. The second best performance is given by the Logistic Regression classifier; the accuracy is $74.5 \%$, the sensitivity is $73.4 \%$, and the specificity is $75.7 \%$. 


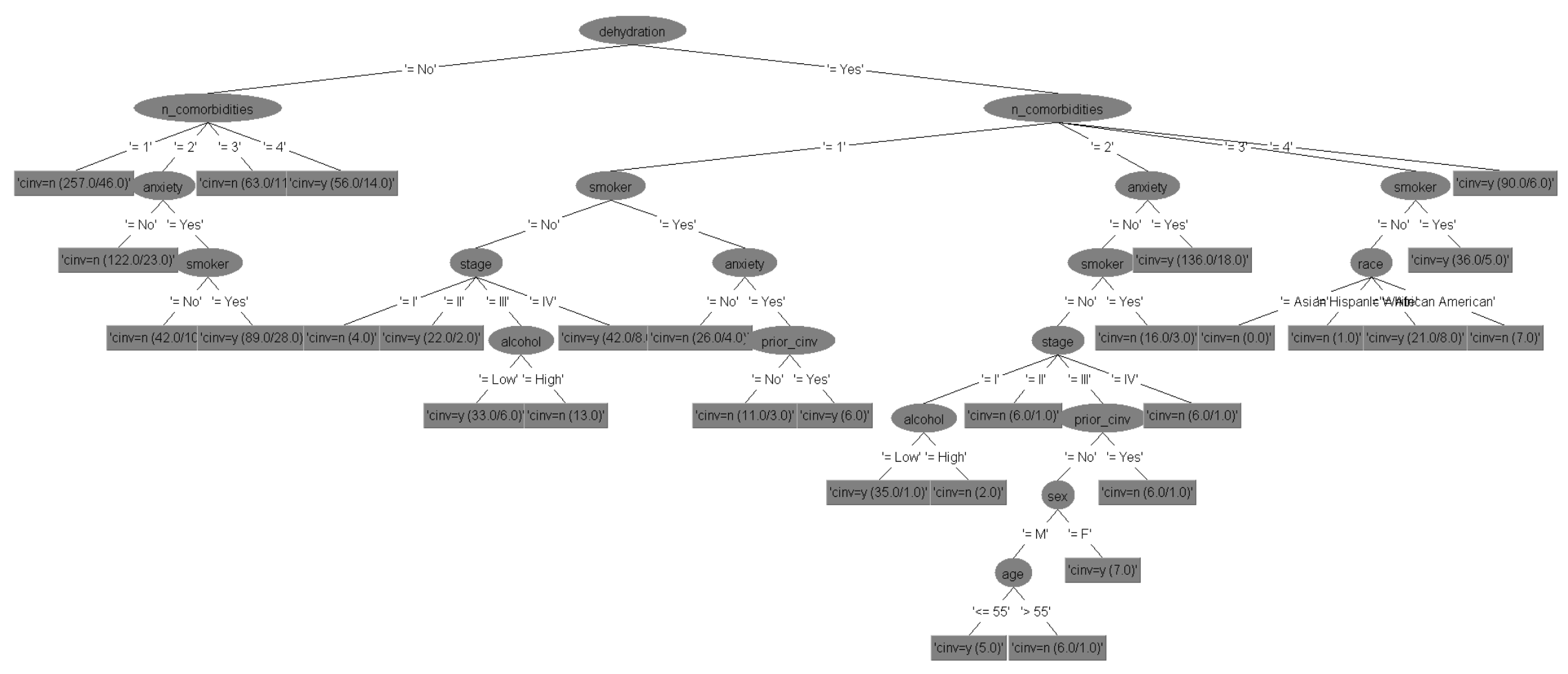

Figure 28: Decision Tree - Delayed, HEC 


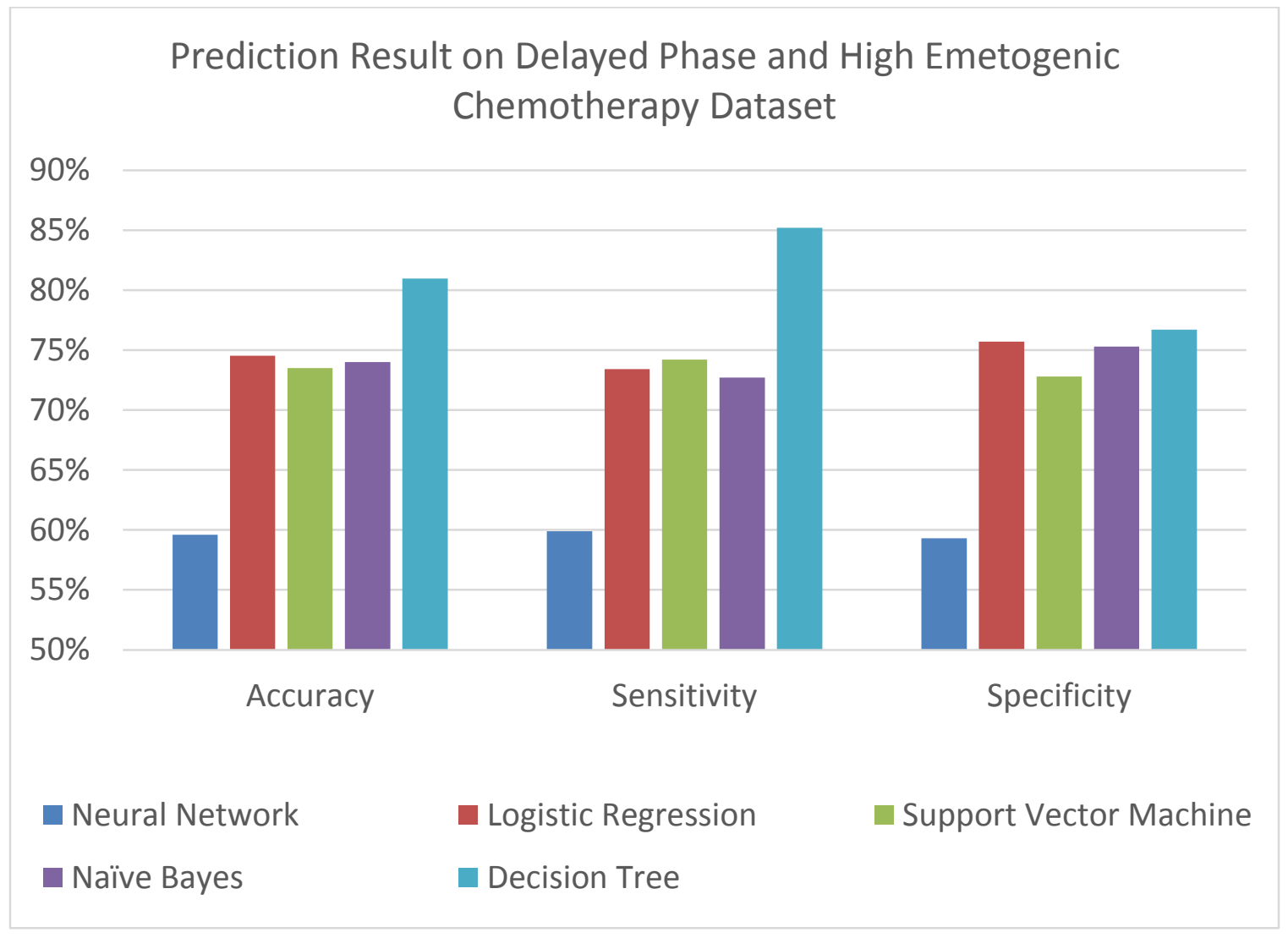

Figure 29: Prediction Result on Delayed Phase and High Emetogenic Chemotherapy Dataset 


\section{CHAPTER 5 : MINING CONTEXT SENSITIVE ASSOCIATIVE CLASSIFICATION RULES}

We further explored the association rule-based classification approach for dynamic prediction. Several association-rule based classification algorithms have been proposed [117]: (1) classification based association (CBA) [118], (2) Classification based on multiple association rules (CMAR) [119], (3) association rule-based classifier with all categories (ARC-AC) [120], (4) classification based on predictive association rules (CPAR) [121], (5) classification based on Atomic Association Rules (CAAR) [122], (6) associative classifier based on positive and negative rules [123], (7) Live and Let Live $\left(\mathrm{L}^{3}\right)$ [124], (8) multi-class and multi-label associative classification approach (MMAC) ,[125] (9) multi-class classification based on association rule approach (MCAR) [126], (10) class based associative classification (CACA) [127], (11) associative classification based on closed frequent itemsets (ACCF) [128], (12) ACN: associative classifier with negative rules (ACN) [129], (13) associative classification based on compactness of rules (ACCR) [130], (14) adaptive associative classification (ADA) [131], and (15) modified multi-class classification based on association rule (MMCAR) [132].

In this chapter, the concept of associative classification with context sensitivity is introduced. The next chapter (Chapter 6) covers the development of a dynamic prediction engine using associative classification rules; we have developed a new classification algorithm using multiple rules. The concept of mining associative classification rules with 
context sensitivity is derived from the concept of association rule mining. As such, in this chapter, the techniques for mining association rules are discussed first followed by the techniques for mining context sensitive associative classification rules.

\subsection{ASSOCIATION RULE MINING}

A very popular method for mining associations among a set of items is the Association Rule Mining (also known as the frequent itemset mining) [133]. Association rule mining (ARM) is a method of identifying associations among a set of items or objects in a database. ARM is also known as frequent itemset mining. The outcome of ARM is association rules - statements of the form $X \rightarrow Y$. Here, $\mathrm{X}$ is the left hand side (LHS) or antecedent and $\mathrm{Y}$ is the right hand side (RHS) or consequent. ARM technique was originally designed to work with a market basket dataset, in which the dataset consists of a set of grocery transactions and a transaction contains a set of items [133,134]. Figure 30 presents an example of a frequent itemset and an association rule derived from this frequent itemset. ARM requires two user input parameters (i.e., support indicating the measure of usefulness, and confidence indicating the measure of certainty) to filter out uninteresting association rules; see section 5.1.3 for more details. 


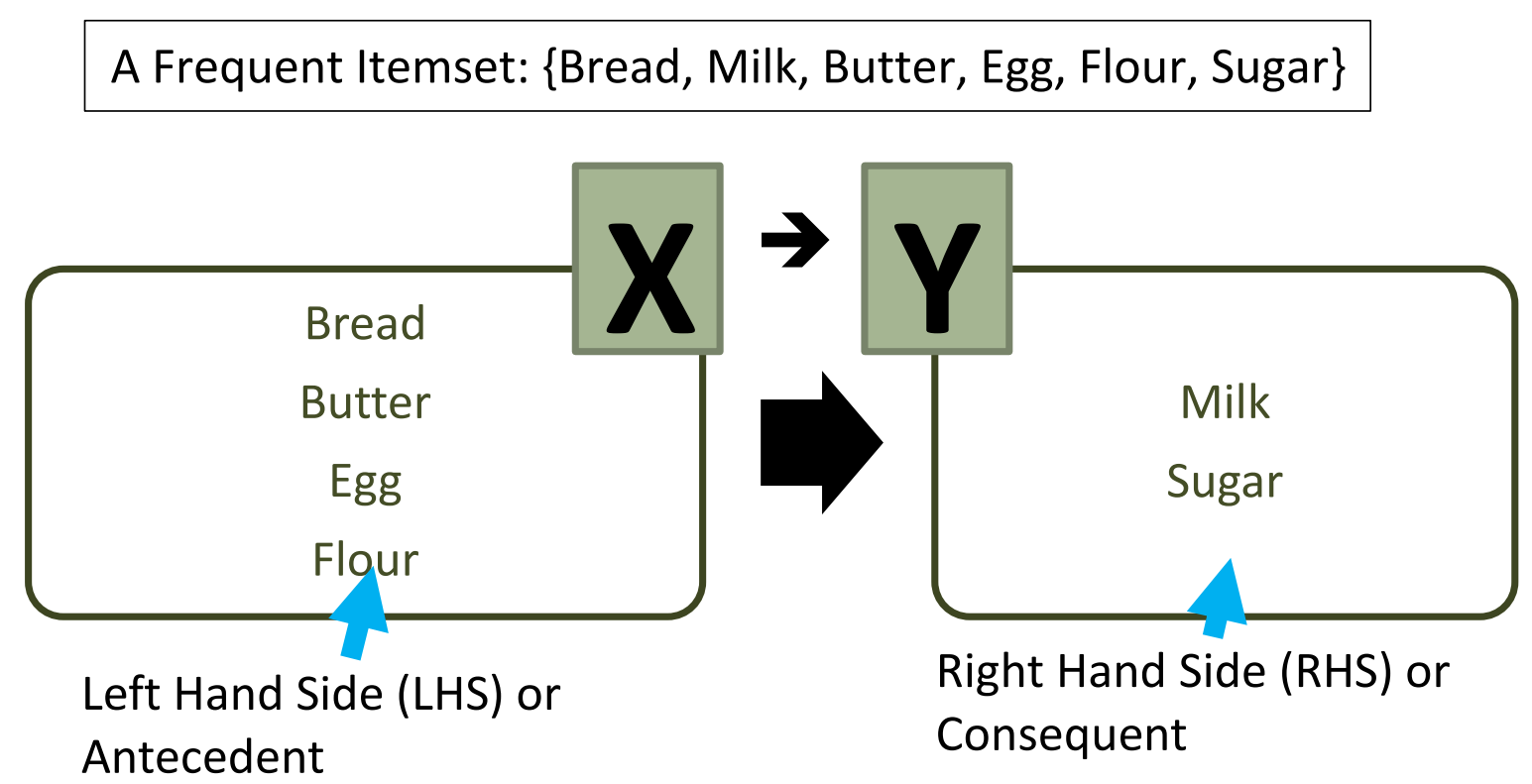

Figure 30: Association Rule Example

ARM was successfully applied in medical informatics research to discover associations among drugs and diseases [135]. It was also found to be useful in text mining [136] and web usage mining [137].

\subsubsection{Apriori}

A novel association mining algorithm is Apriori $[134,138]$. The Apriori algorithm works by generating candidate itemsets and calculating their support counts. In order to calculate the support count of each candidate itemset, a full database scan is required. Thus, the computational performance of the algorithm could be extremely poor especially there are a lot of itemsets. The Apriori algorithm uses prior knowledge of frequent itemsets to eliminate infrequent candidate itemsets. This is known as Apriori property, which states that the subsets of a frequent itemset is also frequent. This property is used for pruning 
infrequent itemsets after candidate generation in every iteration before DB scans. Thus, the pruning step reduces the number of database scans, which leads to considerable performance improvement.

\subsubsection{FP-Growth}

The main performance bottleneck of the Apriori algorithm is the costly generation of a large number of candidate itemsets. An alternative method to the Apriori algorithm is FP-Growth [139], which does not generate candidate itemsets. In FP-Growth, a compact but complete frequent pattern tree (FP-tree) structure is constructed by scanning the database only twice. In the first scan, frequent 1-itemsets are generated. In the second scan, a FP-tree is constructed. Finally, the FP-tree is mined for generating the frequent itemsets.

\subsubsection{Association Rule Quality Measures}

The quality of an association rule is indicated based on several measurements. These are discussed in the following.

\subsubsection{Support}

The support of an association rule is the percentage of transactions that contain the itemset in the DB (Equation 1). This indicates the usefulness of the rule. This is used to filter out uninteresting association rules. If we consider the transaction database in Table 12 , there are total 9 transactions. For the association rule $\{$ Bread, Butter $\} \rightarrow\{$ Sugar $\}$, the itemset $\{$ Bread, Butter, Sugar\} appears 2 times in the database. Thus, the support of the association rule is $22.22 \%(2 / 9)$. 


\section{Support}

$=\frac{\text { Number of transactions that contain an itemset }}{\text { Total Number of transactions (N) }}$

Total Number of transactions $(N)$

\section{Equation 1: Association Rule - Support}

Table 12: Example Transactions for Illustrating Support, Confidence and Lift

\begin{tabular}{|ll|}
\hline ID & Transactions \\
\hline 1 & Bread, Butter, Sugar \\
2 & Butter, Egg \\
3 & Butter, Milk \\
4 & Bread, Butter, Egg \\
5 & Bread, Milk \\
6 & Butter, Milk \\
7 & Bread, Milk \\
8 & Bread, Butter, Milk, Sugar \\
9 & Bread, Butter, Milk \\
\hline
\end{tabular}

\subsubsection{Confidence}

The confidence of an association rule is the conditional probability that the consequent (the right-hand side) will appear given the occurrence of the antecedent (the left-hand side) (Equation 2). In other words, it shows how frequently the consequent occurs in transactions containing antecedent. This indicates the certainty of the rule. This measure is also used to filter out uninteresting association rules. If we consider the transaction database in Table 12, for the association rule $\{$ Bread, Butter $\} \rightarrow\{$ Sugar $\}$, the itemset \{Bread, Butter, Sugar\} is found 2 times and the itemset $\{$ Bread, Butter $\}$ is found 4 times.

Thus, the confidence of the association rule is $50 \%\left(\frac{\text { support }(\{\text { Bread,Butter,Sugar }\})}{\text { support }(\{\text { Bread,Butter }\})}=\frac{\mathbf{2 / 9}}{\mathbf{4 / 9}}=2 / 4\right.$ $=50 \%$. 


\section{Confidence $=\frac{\text { Support }(X \cup Y)}{\text { Support }(X)}$}

\section{Equation 2: Association Rule - Confidence}

\subsubsection{Lift}

The lift of an association rule is the ratio of the support measure of the rule with the multiplication of the support measure of the left hand side or antecedent and the support measure of the right hand side or consequent (Equation 3). This indicates the associativity of the rule. If we consider the transaction database in Table 12, for the association rule $\{$ Bread, Butter $\} \rightarrow\{$ Sugar $\}$, the support of $\{$ Bread, Butter, Sugar $\}$ is $2 / 9$, the support of \{Bread, Butter\} is $4 / 9$ and the support of $\{$ Sugar $\}$ is $2 / 9$. Thus, the lift of the association rule is $\left(\frac{\operatorname{support}(\{\text { Bread,Butter, Sugar }\})}{\operatorname{support}(\{\text { Bread,Butter }\}) * \operatorname{support}(\{\operatorname{Sugar}\})}=\frac{2 / 9}{\frac{4}{9} * \frac{2}{9}}=2.25\right)$.

$$
\text { Lift } \quad=\frac{\operatorname{Support}(X \cup Y)}{\operatorname{Support}(X) * \operatorname{Support}(Y)}
$$

\section{Equation 3: Association Rule - Lift}

The support measure of an itemset can be explained by the concept of probability. Support (X U Y) represents the probability that items in X and items in Y co-occur together, support $(\mathrm{X})$ represents the probability that items in $\mathrm{X}$ co-occur together and support ( $\mathrm{Y}$ ) represents the probability that items in Y co-occur together. If RHS is independent of LHS, the multiplication of their individual probability will be equal to the probability of the itemset. Thus, the lift value becomes 1 representing the independence of LHS and RHS. If 
it is less than 1 , they are negatively correlated. If it is greater than 1 , they are positively correlated. This can be illustrated by using the transaction database in Table 13, where there are more occurrences of the item sugar. For the association rule $\{$ Bread, Butter $\} \rightarrow$ \{Sugar $\}$, the support of $\{$ Bread, Butter, Sugar $\}$ is $4 / 9$, the support of $\{$ Bread, Butter $\}$ is $4 / 9$ and the support of $\{$ Sugar $\}$ is $9 / 9$. Thus, the lift of the association rule is 1 $\left(\frac{\operatorname{support}(\{\text { Bread,Butter,Sugar }\})}{\text { support }(\{\text { Bread,Butter }\}) * \operatorname{support}(\{\text { Sugar }\})}=\frac{\mathbf{4 / 9}}{\frac{\mathbf{4}}{\mathbf{9}} * \mathbf{9}}=1\right)$. In this example, the LHS occurs regardless of RHS.

Table 13: Example of Transactions for Illustrating Independence (Lift=1)

\begin{tabular}{|ll}
\hline \multicolumn{1}{c|}{ Transaction ID } & Transactions \\
\hline 1 & Bread, Butter, Sugar \\
2 & Butter, Egg, Sugar \\
4 & Butter, Milk, Sugar \\
5 & Bread, Butter, Egg, Sugar \\
6 & Bread, Milk, Sugar \\
7 & Butter, Milk, Sugar \\
8 & Bread, Milk, Sugar \\
9 & Bread, Butter, Milk, Sugar \\
\hline
\end{tabular}

\subsection{ASSOCIATION RULE MINING TOOLS}

There are several tools available for association rule mining. However, a single tool does not support all the things needed for experiments. We used two programs: the "arules" package [140] in the R software [141] for generating frequent itemsets, and the FP-growth algorithm [142,143] in WEKA (version 3.6.8) [111] for generating association rules. The reason for using two programs is that the arules package cannot produce association rules 
containing more than one item in the consequent (also known as many-to-many association rules) [144] and WEKA does not produce frequent itemsets. The arules package in $\mathrm{R}$ takes the market basket format as input or any data frame with discrete values in each column can be converted to transactions. In WEKA, the sparse ARFF [145] format is needed for the input of WEKA FP-Growth algorithm, where each attribute value pair is represented as variable.

\subsection{EXAMPLES OF ASSOCIATION RULE MINING}

\subsubsection{Mining Healthcare Data}

In healthcare research, ARM has unearthed important associations among drugs and diseases [135]. For example, Chen et al. (2003) [146] used ARM to discover coprescription patterns in the National Health Insurance Research Database (NHIRD) managed by Taiwan National health Insurance. An example association rule that was discovered by the study is $\{$ Muscle relaxants, centrally acting drugs $\} \rightarrow$ \{antacid\} ( support $=3.8 \%$, confidence $=77.5 \%$ ), which indicates that $77.5 \%$ of patients who take muscle relaxants and centrally acting drugs take antacid and the portion of the transactions in the database that meet the association rule is $3.8 \%$. The analysis by Tai \& Chiu (2009) [43] on NHIRD database revealed important association of diseases with Attention Deficit/Hyperactivity Disorder (ADHD) that is a highly common chronic behavior condition in childhood. 


\subsubsection{Mining Association of PubMed Search Tags}

The author (ASMM) applied association rule mining technique on a large day-long PubMed log dataset [147] in order to discover hidden associations among search tags $[148,149]$. The dataset contains nearly 3 million search queries [150]. We discovered association rules both at the query level [148] and at the session level [149]. In Mosa and Yoo (2014) [149], we applied ARM technique for identifying the associations or cooccurrences of search tags in the search sessions. In this study, a search session is considered as a transaction and a set of search tags in the session is considered as an itemset. The output of ARM is a set of association rules of the form $X \rightarrow Y$ (support, confidence); that is, if a user uses a set of search tags in $X$ (the antecedent) also uses a set of search tags in $Y$ (the consequent). In this study, we used the FP-Growth association mining algorithm.

We performed ARM in two steps. In the first step, we generated frequent itemsets with 0.02 (i.e., $2 \%$ ) of the minimum support. The minimum support was set to 0.02 because the dataset is very sparse. The total number of frequent itemsets was 131 . We analyzed these 131 frequent itemsets to identify the maximal itemsets using the "is.maximal" function of the arules package resulting 12 maximal itemsets. A maximal itemset is a frequent itemset that have no superset among the frequent itemsets [151]. For example, if $\mathrm{A}=\{\mathrm{a}, \mathrm{b}, \mathrm{c}, \mathrm{d}\}, \mathrm{B}=\{\mathrm{a}, \mathrm{b}, \mathrm{c}\}$, and $\mathrm{C}=\{\mathrm{b}, \mathrm{c}, \mathrm{d}\}$ are three frequent itemsets, where $B \subset A$ and $C \subset A$, then $\mathrm{A}$ is the maximal itemset. We know from the apriori principal that any subset of a frequent itemset is also frequent. So, we choose only the maximal itemsets because their subsets are redundant for our particular goal. We presented a total of 8 maximal frequent itemsets (out of 12) in Table 14 and Table 15. The remaining four maximal frequent itemsets are not presented because two itemsets are of size one and the 
other two itemsets do not have any strong association rules. In the second step, we generated the association rules $(X \rightarrow Y)$ with 0.5 (i.e., 50\%) of the minimum confidence. The association rules were generated using all the frequent itemsets. The total number of association rules was 515 . Out of the 515 association rules, only the rules consisting of maximal frequent itemsets were extracted.

Among the 8 maximal frequent itemsets, three of them consist of purely informational tags (maximal frequent itemset no. 1, 2, and 3 in Table 14). Search tags are categorized into informational or navigational based on their underlying intent. Refer to our recent article Mosa and Yoo (2013) [148] for the complete list of informational and navigational search tags. Itemset no. 1 have six search tags in which two of them are topical search fields (i.e., [MESH TERMS] and [MESH MAJOR TOPIC] tags), one of them is for searching a word or number in various text fields (i.e., [TEXT WORD] tag), and the reaming three tags represent filtering of search results based on: the language of the article (i.e., [LANGUAGE] tag) and/or the publication type of the article (i.e., [PUBLICATION TYPE] tag) and/or the subject, citation status or journal category of the article (i.e., [SUBSET] tag). We found a total of 48 association rules consisting of these six search tags (itemset no. 1) satisfying the minimum confidence of $50 \%$ but we present only 12 association rules (R01 to R12 in Table 14) having more than $90 \%$ of confidence. Thus, all these search tags in itemset no. 1 and its subsets are very strongly associated with one another and commonly used within the same search sessions. 
Table 14. Association Rules Consisting of Informational Tags only

\begin{tabular}{|c|c|c|}
\hline $\begin{array}{l}\text { Rule } \\
\text { ID }\end{array}$ & Association Rule & Conf. \\
\hline & $\begin{array}{c}\text { Itemset No. } 1 \text { (Supp. = 0.032): } \\
\text { [LANGUAGE], [MESH MAJOR TOPIC], [MESH TERMS], [PUBLICATION } \\
\text { TYPE], [SUBSET], [TEXT WORD] }\end{array}$ & \\
\hline R01 & $\begin{array}{l}\text { [MESH TERMS], [PUBLICATION TYPE], [MESH MAJOR TOPIC], [TEXT } \\
\text { WORD], [SUBSET] == [LANGUAGE] }\end{array}$ & 0.99 \\
\hline R02 & $\begin{array}{l}\text { [LANGUAGE], [PUBLICATION TYPE], [MESH MAJOR TOPIC], [TEXT WORD], } \\
\text { [SUBSET] ==> [MESH TERMS] }\end{array}$ & 0.98 \\
\hline R03 & $\begin{array}{l}\text { [MESH TERMS], [LANGUAGE], [PUBLICATION TYPE], [MESH MAJOR } \\
\text { TOPIC], [TEXT WORD] ==> [SUBSET] }\end{array}$ & 0.97 \\
\hline R04 & $\begin{array}{l}\text { [PUBLICATION TYPE], [MESH MAJOR TOPIC], [TEXT WORD], [SUBSET] ==> } \\
\text { [MESH TERMS], [LANGUAGE] }\end{array}$ & 0.97 \\
\hline R05 & $\begin{array}{l}\text { [MESH TERMS], [PUBLICATION TYPE], [MESH MAJOR TOPIC], [TEXT } \\
\text { WORD] ==> [LANGUAGE], [SUBSET] }\end{array}$ & 0.96 \\
\hline R06 & $\begin{array}{l}\text { [MESH TERMS], [LANGUAGE], [MESH MAJOR TOPIC], [TEXT WORD], } \\
{[\text { SUBSET] }==>\text { [PUBLICATION TYPE] }}\end{array}$ & 0.95 \\
\hline R07 & $\begin{array}{l}\text { [LANGUAGE], [PUBLICATION TYPE], [MESH MAJOR TOPIC], [TEXT WORD] } \\
==>\text { [MESH TERMS], [SUBSET] }\end{array}$ & 0.94 \\
\hline R08 & $\begin{array}{l}\text { [MESH TERMS], [MESH MAJOR TOPIC], [TEXT WORD], [SUBSET] ==> } \\
\text { [LANGUAGE], [PUBLICATION TYPE] }\end{array}$ & 0.94 \\
\hline R09 & $\begin{array}{l}\text { [LANGUAGE], [MESH MAJOR TOPIC], [TEXT WORD], [SUBSET] ==> [MESH } \\
\text { TERMS], [PUBLICATION TYPE] }\end{array}$ & 0.93 \\
\hline R10 & $\begin{array}{l}\text { [PUBLICATION TYPE], [MESH MAJOR TOPIC], [TEXT WORD] ==> [MESH } \\
\text { TERMS], [LANGUAGE], [SUBSET] }\end{array}$ & 0.92 \\
\hline R11 & $\begin{array}{l}\text { [MESH MAJOR TOPIC], [TEXT WORD], [SUBSET] ==> [MESH TERMS], } \\
\text { [LANGUAGE], [PUBLICATION TYPE] }\end{array}$ & 0.91 \\
\hline \multirow[t]{2}{*}{ R12 } & $\begin{array}{l}\text { [MESH TERMS], [LANGUAGE], [MESH MAJOR TOPIC], [TEXT WORD] ==> } \\
\text { [PUBLICATION TYPE], [SUBSET] }\end{array}$ & 0.90 \\
\hline & $\begin{array}{c}\text { Itemset No. } 2(\text { Supp. }=0.038) \text { : } \\
\text { [TITLE ABSTRACT], [PUBLICATION TYPE] }\end{array}$ & \\
\hline \multirow[t]{2}{*}{ R13 } & [TITLE ABSTRACT] ==> [PUBLICATION TYPE] & 0.60 \\
\hline & $\begin{array}{l}\text { Itemset No. } 3(\text { Supp. }=0.020) \text { : } \\
\text { [SUBHEADING], [MESH TERMS] }\end{array}$ & \\
\hline $\mathrm{R} 14$ & {$[$ SUBHEADING] $==>$ [MESH TERMS $]$} & 0.89 \\
\hline
\end{tabular}


We found five maximal frequent itemsets (out of eight) that contains purely navigational/bibliographic search tags (maximal itemset no. 4, 5, 6, 7 and 8 in Table 15). The intent of navigational or bibliographic searches is to identify specific documents. Maximal frequent itemset no. 6, 7 and 8 have five items in each itemset, in which three of them are common (i.e., [PUBLICATION DATE], [PAGINATION], [VOLUME]). The search tag [ISSUE] is common in itemset no 6 and 7, the tag [JOURNAL] is common in itemset no. 7 and 8 , and the tag [AUTHOR] is common in itemset no. 6 and 8 . Thus, itemset no 6,7 and 8 are subset of \{[PUBLICATION DATE], [PAGINATION], [VOLUME], [ISSUE], [JOURNAL], [AUTHOR]\}. We discovered 7, 7 and 8 association rules for itemset no. 6, 7 and 8, respectively (Table 15). The confidence of these rules ranges from $53 \%$ to $97 \%$. So these six search fields are strongly correlated. A search query having these six search fields will retrieve only a single article. 
Table 15. Association Rules Consisting of Navigational Tags Only

\begin{tabular}{|c|c|c|}
\hline $\begin{array}{l}\text { Rule } \\
\text { ID }\end{array}$ & Association Rule & Conf. \\
\hline & $\begin{array}{l}\text { Itemset No. } 4 \text { (Supp. }=0.021) \text { : } \\
\text { [FIRST AUTHOR NAME], [PUBLICATION DATE }]\end{array}$ & \\
\hline \multirow[t]{2}{*}{ R01 } & [FIRST AUTHOR NAME] ==> [PUBLICATION DATE] & 0.77 \\
\hline & $\begin{array}{l}\text { Itemset No. } 5 \text { (Supp. }=0.035): \\
\text { [ENTREZ DATE], [PUBLICATION DATE] }\end{array}$ & \\
\hline \multirow[t]{2}{*}{ R02 } & [ENTREZ DATE] ==> [PUBLICATION DATE] & 0.063 \\
\hline & $\begin{array}{l}\text { Itemset No. } 6 \text { (Support=0.021): } \\
\text { [PUBLICATION DATE], [PAGINATION], [AUTHOR], [ISSUE], [VOLUME] }\end{array}$ & \\
\hline R03 & [PUBLICATION DATE], [PAGINATION], [AUTHOR], [ISSUE] $==>$ [VOLUME] & 0.97 \\
\hline R04 & [PUBLICATION DATE], [VOLUME], [AUTHOR], [ISSUE] ==> [PAGINATION] & 0.89 \\
\hline R05 & [PAGINATION], [VOLUME], [AUTHOR], [ISSUE] ==> [PUBLICATION DATE] & 0.87 \\
\hline R06 & [PUBLICATION DATE], [AUTHOR], [ISSUE] ==> [PAGINATION], [VOLUME] & 0.87 \\
\hline R07 & [PAGINATION], [AUTHOR], [ISSUE] ==> [PUBLICATION DATE], [VOLUME] & 0.85 \\
\hline R08 & [VOLUME], [AUTHOR], [ISSUE] ==> [PUBLICATION DATE], [PAGINATION] & 0.77 \\
\hline \multirow[t]{2}{*}{ R09 } & [AUTHOR], [ISSUE] $==>$ [PUBLICATION DATE], [PAGINATION], [VOLUME] & 0.74 \\
\hline & $\begin{array}{l}\text { Itemset No. } 7 \text { (Supp. }=0.037 \text { ): } \\
\text { [PUBLICATION DATE], [JOURNAL], [PAGINATION], [ISSUE], [VOLUME] }\end{array}$ & \\
\hline R10 & [PUBLICATION DATE], [JOURNAL], [PAGINATION], [ISSUE] ==> [VOLUME] & 0.98 \\
\hline R11 & [PUBLICATION DATE], [JOURNAL], [VOLUME], [ISSUE] ==> [PAGINATION] & 0.89 \\
\hline R12 & [PUBLICATION DATE], [JOURNAL], [ISSUE] ==> [PAGINATION], [VOLUME] & 0.86 \\
\hline R13 & [JOURNAL], [PAGINATION], [VOLUME], [ISSUE] ==> [PUBLICATION DATE] & 0.81 \\
\hline R14 & [JOURNAL], [PAGINATION], [ISSUE] ==> [PUBLICATION DATE], [VOLUME] & 0.79 \\
\hline R15 & [JOURNAL], [VOLUME], [ISSUE] ==> [PUBLICATION DATE], [PAGINATION] & 0.71 \\
\hline \multirow[t]{2}{*}{ R16 } & [JOURNAL], [ISSUE] ==> [PUBLICATION DATE], [PAGINATION], [VOLUME] & 0.68 \\
\hline & $\begin{array}{l}\text { Itemset No. } 8 \text { (Support=0.038): } \\
\text { [PUBLICATION DATE], [JOURNAL], [PAGINATION], [AUTHOR], [VOLUME] }\end{array}$ & \\
\hline R17 & $\begin{array}{l}\text { [PUBLICATION DATE], [JOURNAL], [PAGINATION], [AUTHOR] ==> } \\
\text { [VOLUME] }\end{array}$ & 0.85 \\
\hline R18 & $\begin{array}{l}\text { [PUBLICATION DATE], [JOURNAL], [VOLUME], [AUTHOR] ==> } \\
\text { [PAGINATION] }\end{array}$ & 0.85 \\
\hline R19 & $\begin{array}{l}\text { [JOURNAL], [PAGINATION], [VOLUME], [AUTHOR] ==> [PUBLICATION } \\
\text { DATE] }\end{array}$ & 0.82 \\
\hline R20 & $\begin{array}{l}\text { [JOURNAL], [VOLUME], [AUTHOR] ==> [PUBLICATION DATE], } \\
\text { [PAGINATION] }\end{array}$ & 0.69 \\
\hline R21 & $\begin{array}{l}\text { [PUBLICATION DATE], [PAGINATION], [VOLUME], [AUTHOR] ==> } \\
\text { [JOURNAL] }\end{array}$ & 0.65 \\
\hline R22 & $\begin{array}{l}\text { [PUBLICATION DATE], [VOLUME], [AUTHOR] ==> [JOURNAL], } \\
\text { [PAGINATION] }\end{array}$ & 0.55 \\
\hline R23 & $\begin{array}{l}\text { [PAGINATION], [VOLUME], [AUTHOR] ==> [PUBLICATION DATE], } \\
\text { [JOURNAL] }\end{array}$ & 0.53 \\
\hline R24 & $\begin{array}{l}\text { [PUBLICATION DATE], [PAGINATION], [AUTHOR] ==> [JOURNAL], } \\
\text { [VOLUME] }\end{array}$ & 0.53 \\
\hline
\end{tabular}

The association rules in Table 14 and Table 15 are useful because advance features such as search tags are used by experienced users [147]. The rules can be used to develop an intelligent PubMed search interface. For example, PubMed can automatically 
recommend the use of certain tags based on the other tags used in the query. This intelligent interface will help the users to build a query using proper search tags and reduce the frequency of query refinement.

\subsection{ASSOCIATION RULE VISUALIZATION}

We also extended an association rule visualization software for 3D visualization of association rules with multiple items in the consequent. The association rules presented in Table 14 are visualized in Figure 31 using the "Association Rule Viewer" (ARV) software [152] that implements the novel 3D visualization technique described in Wong et al. (1999) [136]. We also visualized the association rules presented in Table 15 in Figure 32. The association rules are visualized in a checkerboard-like 2D matrix where one association rule is presented in one column; a green cell represents that the corresponding item belongs to the antecedent set $(X)$ and a red cell represents that the corresponding item belongs to the consequent set $(Y)$. The support and confidence measures are visualized as two bar charts along the Z-axis. For example, R12 in Figure 31 visualizes the rule: $\{[\mathrm{MESH}$ TERMS], [LANGUAGE], [MESH MAJOR TOPIC], [TEXT WORD] == [PUBLICATION TYPE], [SUBSET]\} (support: 3.2\% and confidence: 90\%). The ARV software can visualize only many-to-one association rules so we modified the source code of the software for visualizing many-to-many association rules. 


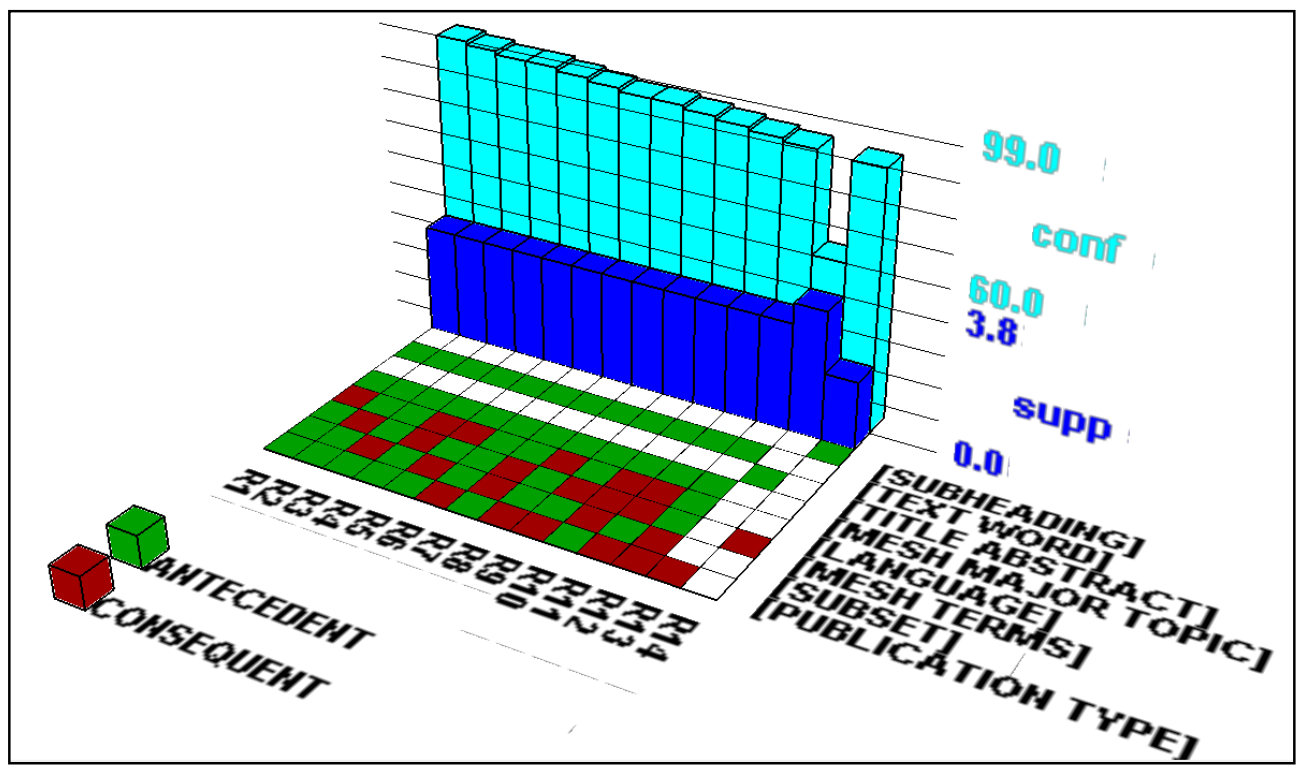

Figure 31. Visualization of association rules consisting of informational tags only

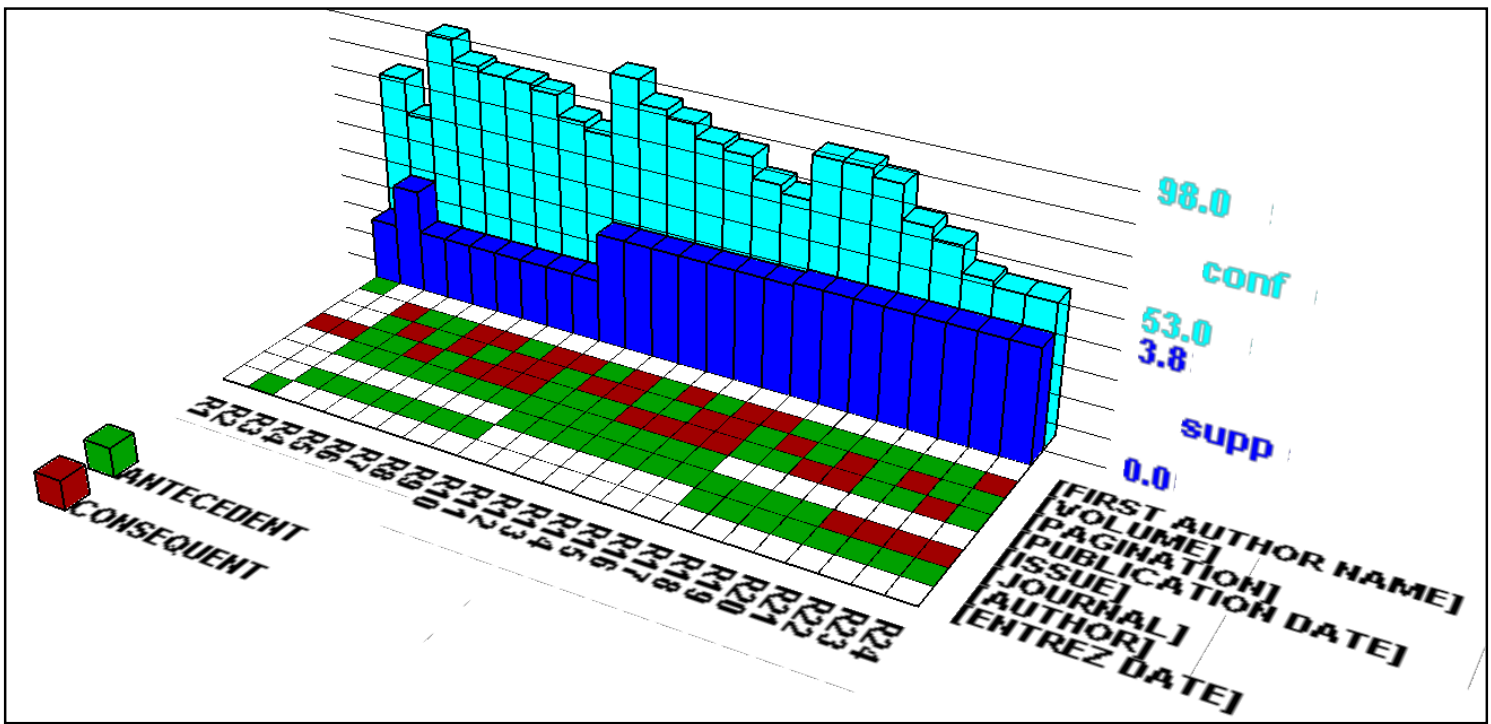

Figure 32. Visualization of association rules consisting of navigational/bibliographic tags only 


\subsection{DATA REPRESENTATION AND ALGORITHM}

In the association mining experiment on PubMed log (presented in $[148,153]$ ), we exploited, the most widely-used association mining algorithm, Apriori in WEKA with the minimum support $=0.02$ and the minimum confidence $=0.5$. This experiment was conducted on a computer with two Intel Xeon CPUs (at 3.00 GHz) and 24.0 GB RAM. The Apriori algorithm was run for more than five full days consuming more than 20GB system memory, but we were unable to get a result using the algorithm. To tackle this problem, we converted the ARFF input file into a spare ARFF [51] in which only positive (here, "Y") values are stored. The spare format significantly reduced the file size from 6.32 MB to $1.55 \mathrm{MB}$. Then, we used the FP-Growth algorithm because it was proven to be more efficient than Apriori (while Apriori generates a lot of candidate itemsets, FP-Growth does not) and because, more importantly, it generates and store only positive rules containing "Y" values. We got a result (a set of association rules) within 5 seconds (we used the same support and confidence values). We would like to stress that selecting a right data format and algorithm could be critical to successful data mining.

\subsection{ASSOCIATION RULE MINING STEPS}

The association rules are mined in two steps (Figure 33); First, all frequent patterns are generated based on a user-defined support parameter. Second, association rules are identified from the frequent patterns based on a user-defined confidence parameter. With the decrease of support count, the number of association rule generated could be increased exponentially depending on confidence; Figure 34 presents the number of association rule generated using our CINV dataset (containing all 6,124 records from both the acute and 
the delayed phase datasets). The processing time of the FP-Growth algorithm is much lower than that of the Apriori algorithm (Figure 35).

Our main goal was to use association rules for classification. We developed a novel algorithm for mining association rules that will be used for classification. The concept of associative classification and the novel technique for direct mining of associative classification rules are discussed in the following sections.
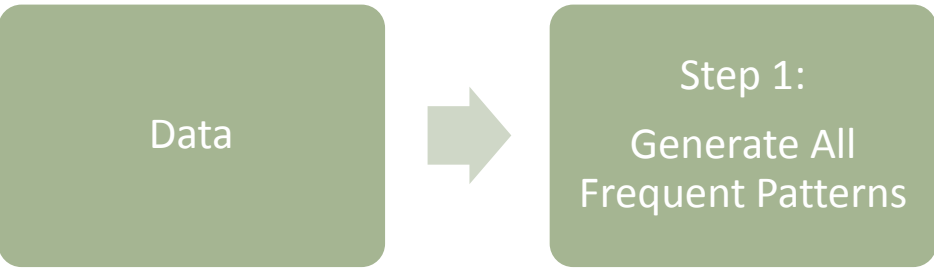

\section{Step 2:}

Generate All

possible

association Rules

Figure 33: Association Rule Mining Steps

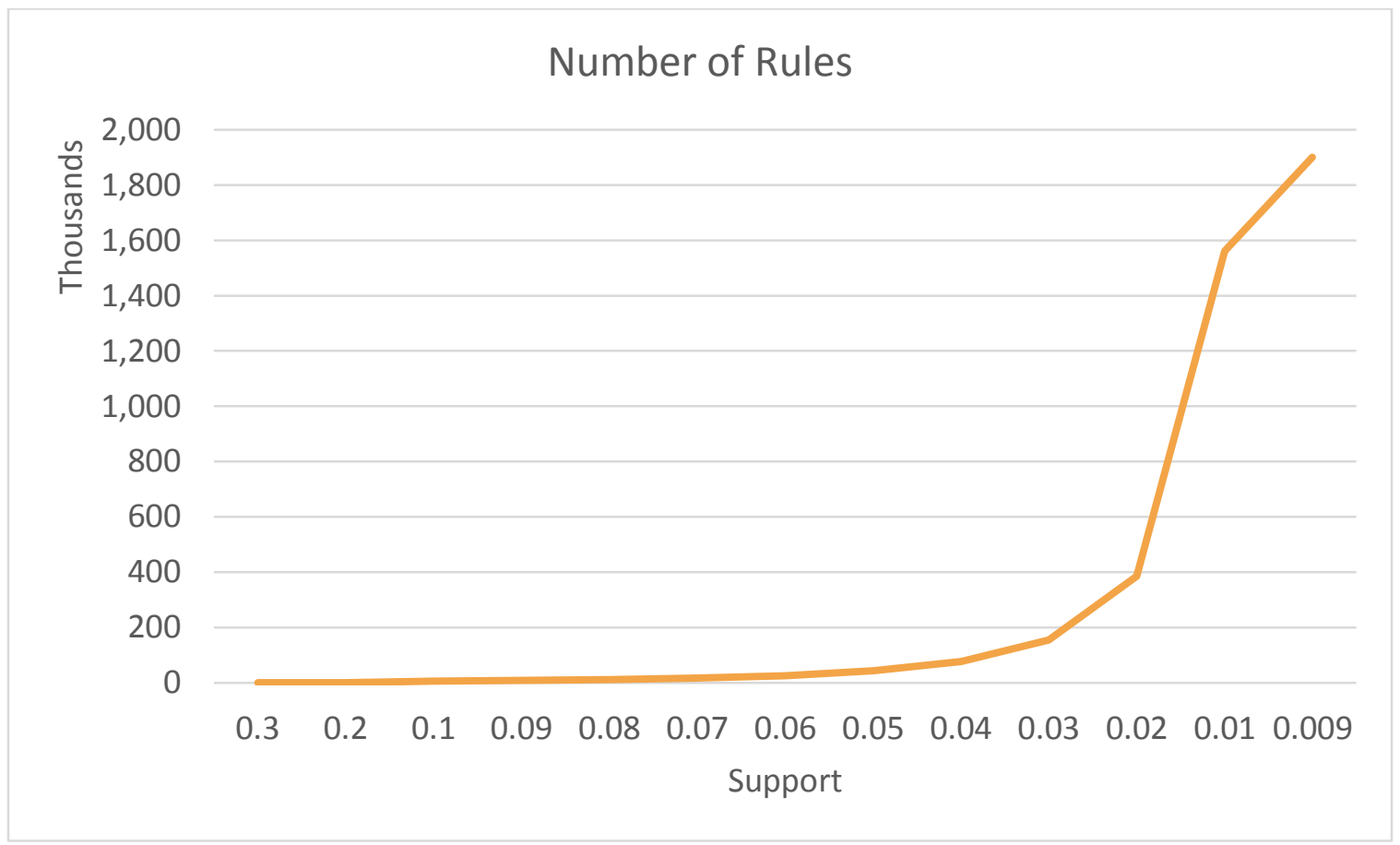

Figure 34: Number of Association Rule Growth Chart 


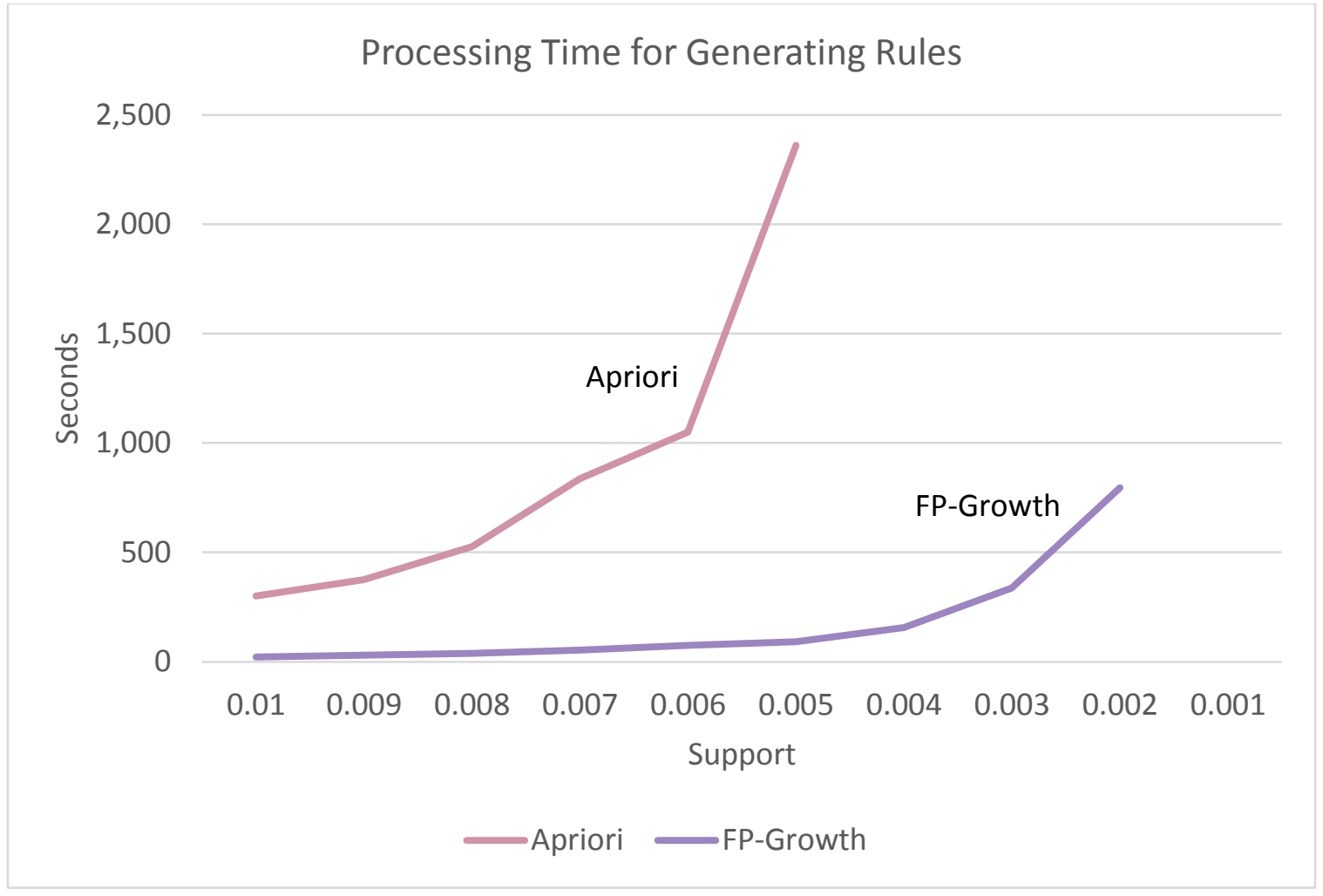

Figure 35: Processing Time for Generating Association Rules

\subsection{ASSOCIATIVE CLASSIFICATION RULES}

Associative classification rules are a subset of association rules where the RHS or consequent of the rule contains only the class/dependent variable along with its label (Figure 36). In order to predict CINV based on patient-related risk factors, we would like to mine all the rules where a set of the risk factors are presented in the LHS of the rule and the occurrence of CINV is presented in the RHS of the rule.; for example: $\{$ Dehydration $=$ Yes, Anxiety $=$ Yes $\} \rightarrow\{\mathrm{CINV}=$ Yes $\}$ 


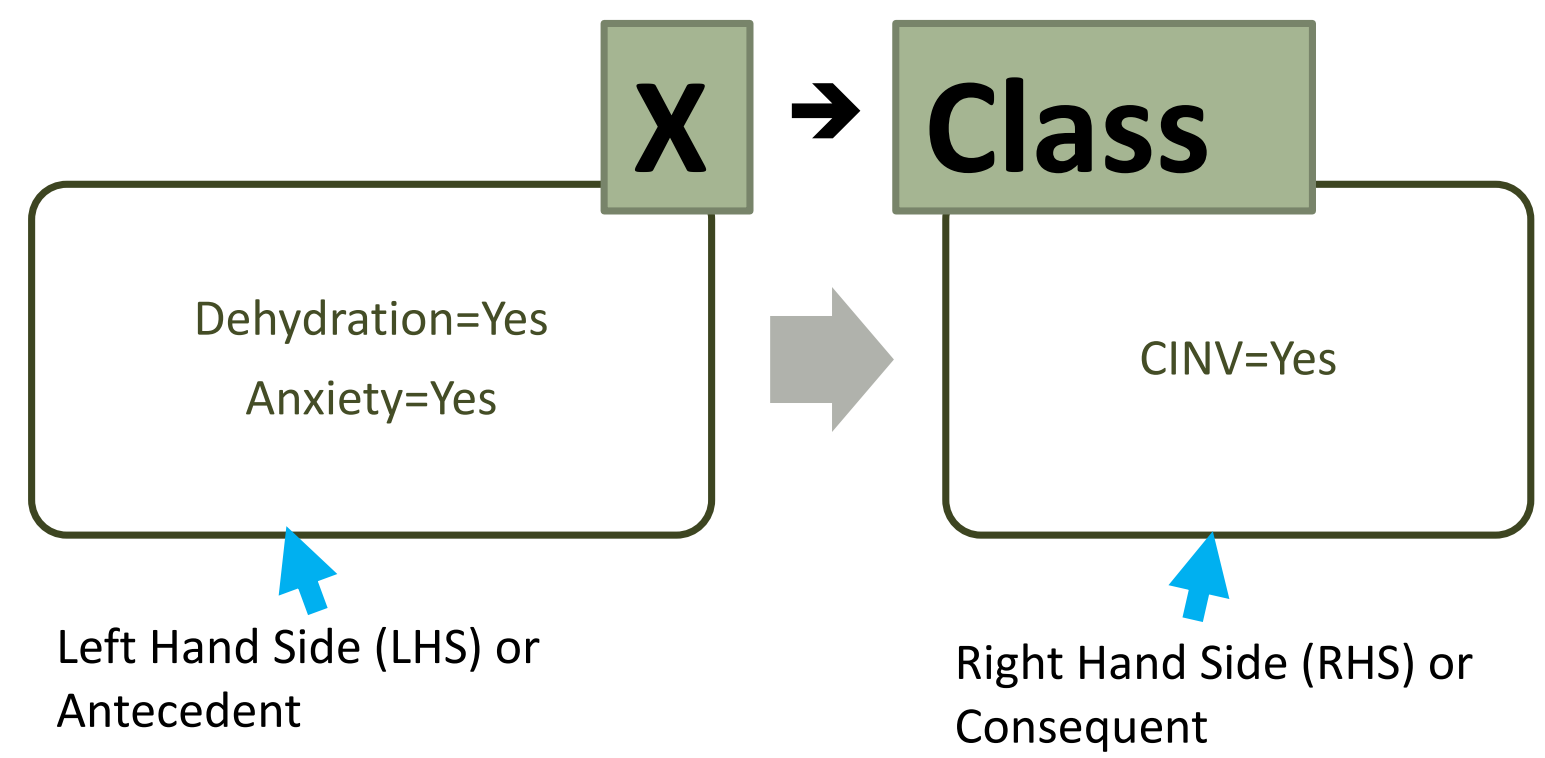

Figure 36: Associative Classification Rule

\subsection{CONTEXT SENSITIVE MINING}

For the problem of CINV prediction, there are three treatment groups and two independent phases of CINV (see Chapter 3 for the data collection plan). As a result, there are six groups of data (Figure 37): (1) low emetogenic chemotherapy - acute phase, (2) low emetogenic chemotherapy - delayed phase, (3) moderate emetogenic chemotherapy acute phase, (4) moderate emetogenic chemotherapy - delayed phase, (5) high emetogenic chemotherapy - acute phase, (6) high emetogenic chemotherapy - delayed phase. In Chapter 4, we presented the data mining results on six contexts independently so that data in one context is not used for prediction in another context. In this section, we are introducing the concept of context sensitivity in the prediction process named as context sensitive prediction. For context sensitive prediction using association rules, we introduce 
a term context sensitive associative classification rules, which is described in the next paragraph.

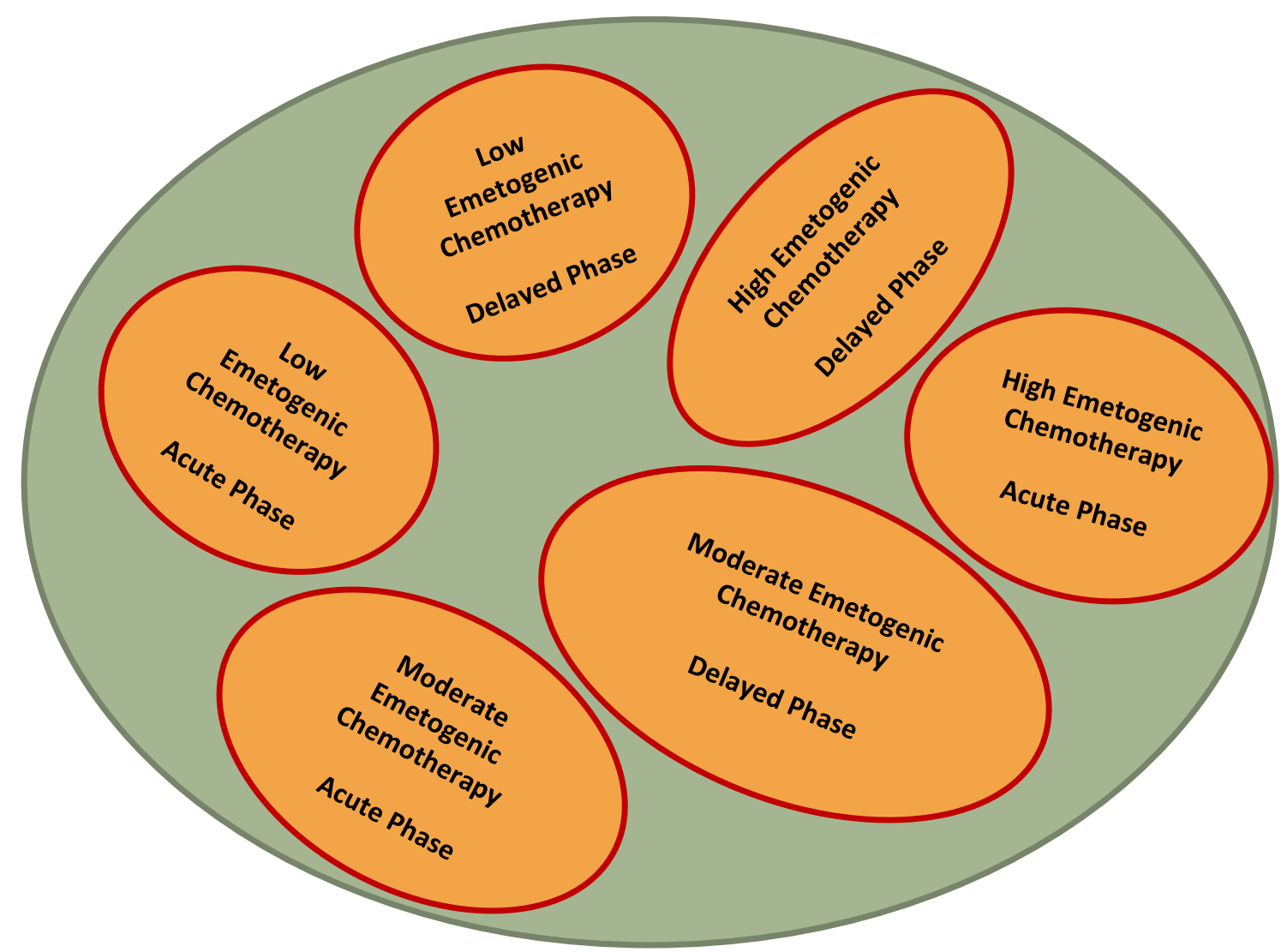

Figure 37: Example of Contexts from the CINV Dataset

Figure 38 presents an example of a context sensitive associative classification rule.

Here, context elements are appended to the items in the LHS. For example, in the association rule (CINV Phase=Acute, Emetogenecity=HEC) (Dehydration=Yes, Anxiety=Yes) $\rightarrow$ CINV=Yes, (CINV Phase=Acute, Emetogenecity=HEC) presents contexts, (Dehydration-Yes, Anxiety=Yes) presents risk factors (i.e., items) and CINV=Yes presents the class. 


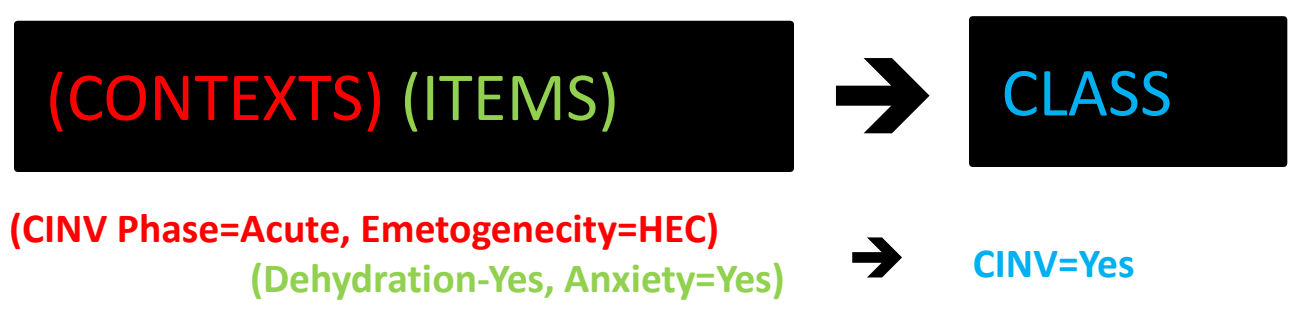

Figure 38: Context Sensitive Associative Classification Rule

The quality measure (support, confidence and lift) of context sensitive associative classification rules can be computed either in global context or in local context. In global context, measures are calculated based on the entire dataset. In local context, the measures are calculated based on a subset of the dataset that fits within the context. The context sensitivity should be considered when a dataset is highly imbalanced in class. The CINV prediction problem is a good example; see Chapter 3 for class imbalance issue. In this study, our dataset was made class-balanced for better comparison between classes. In order to investigate the impact of the quality measures in a class imbalanced situation, we created an imbalanced dataset from the balanced dataset. Table 16 presents the quality measures from the imbalanced dataset for both global and local contexts. For low emetogenic chemotherapy, we observed that some rules show weak associativity (lift <1) within local context whereas the same rules show strong associativity within global context (lift > 1). As such, we considered context sensitive measures for context sensitive mining; see Equation 4 for context sensitive support, Equation 5 for context sensitive confidence and Equation 6 for context sensitive lift. 
Table 16: Examples of Context Sensitive Associative Classification Rules from a Class Imbalanced Data

\begin{tabular}{|c|c|c|c|c|c|c|}
\hline \multirow[b]{2}{*}{ Association Rules } & \multicolumn{3}{|c|}{$\begin{array}{l}\text { Measures are } \\
\text { calculated based on } \\
\text { the entire dataset }\end{array}$} & \multicolumn{3}{|c|}{$\begin{array}{l}\text { Measures are } \\
\text { calculated based on a } \\
\text { subset for each context }\end{array}$} \\
\hline & $\begin{array}{l}\text { Supp } \\
\text { ort }\end{array}$ & $\begin{array}{l}\text { Confid } \\
\text { ence }\end{array}$ & Lift & $\begin{array}{l}\text { Supp } \\
\text { ort }\end{array}$ & $\begin{array}{l}\text { Confid } \\
\text { ence }\end{array}$ & Lift \\
\hline $\begin{array}{l}\text { (CINV Phase=Acute, Emetogenecity=HEC) } \\
(\text { Anxiety=Yes }) \rightarrow C I N V=Y e s\end{array}$ & 0.09 & 0.95 & 1.54 & 0.70 & 0.95 & 1.14 \\
\hline $\begin{array}{l}\text { (CINV Phase=Delayed, Emetogenecity=HEC) } \\
(\text { Anxiety=Yes) } \rightarrow \text { CINV=Yes }\end{array}$ & 0.06 & 0.77 & 1.24 & 0.43 & 0.77 & 1.06 \\
\hline $\begin{array}{l}\text { (CINV Phase=Acute, Emetogenecity=MEC) } \\
\text { (Anxiety=Yes) } \rightarrow \text { CINV=Yes }\end{array}$ & 0.18 & 0.94 & 1.52 & 0.62 & 0.94 & 1.25 \\
\hline $\begin{array}{l}\text { (CINV Phase=Delayed, Emetogenecity=MEC) } \\
(\text { Anxiety=Yes) } \rightarrow \text { CINV=Yes }\end{array}$ & 0.12 & 0.81 & 1.31 & 0.42 & 0.81 & 1.30 \\
\hline $\begin{array}{l}\text { (CINV Phase=Acute, Emetogenecity=LEC) } \\
(\text { Anxiety }=\text { Yes }) \rightarrow \text { CINV=No }\end{array}$ & 0.02 & 0.72 & $1.89^{a}$ & 0.21 & 0.72 & $0.80^{b}$ \\
\hline $\begin{array}{l}\text { (CINV Phase=Delayed, Emetogenecity=LEC) } \\
(\text { Anxiety=Yes) } \rightarrow \text { CINV=No }\end{array}$ & 0.03 & 0.86 & $2.26^{a}$ & 0.41 & 0.86 & $0.98^{c}$ \\
\hline \multicolumn{7}{|c|}{$\begin{array}{l}\text { a Strong association with respect to the entire dataset } \\
\text { b Weak association with respect to the context } \\
\text { c Independent association with respect to the context }\end{array}$} \\
\hline
\end{tabular}

$$
\text { Support } \quad=\frac{\text { Number of transactions that contain an itemset }}{\text { Total Number of transactions }(N)}
$$

Context Sensitive Number of transactions containing an itemset within a context

Support (CSS) $=\frac{\text { Total number of transaction within this context }}{\text { Sum }}$

Equation 4: Context Sensitive Support 


$$
\text { Confidence } \quad=\frac{\text { Support }(\text { CONTEXTS U ITEMS U CLASS })}{\text { Support }(\text { CONTEXTS } U \text { ITEMS })}
$$

\section{$\begin{aligned} & \text { Context Sensitive } \\ & \text { Confidence }\end{aligned}=\frac{\text { CS Support }(\text { CONTEXTS U ITEMS U CLASS })}{\text { CS Support }(\text { CONTEXTS } U \text { ITEMS })}$}

\section{Equation 5: Context Sensitive Confidence}

\begin{tabular}{|c|c|}
\hline Lift & $=\frac{\text { Support }(\text { CONTEXTS U ITEMS U CLASS })}{\text { Support }(\text { CONTEXTS U ITEMS }) * \text { Support }(\text { CLASS })}$ \\
\hline $\begin{array}{l}\text { Context Sensitive } \\
\text { Lift }\end{array}$ & $=\frac{\text { CS Support (CONTEXTS U ITEMS U CLASS) }}{\text { CS Support (CONTEXTS U ITEMS) } * \text { CS Support (CONTEXTS U CLASS) }}$ \\
\hline
\end{tabular}

Equation 6: Context Sensitive Lift

\subsection{MINING CONTEXT SENSITIVE ASSOCIATIVE CLASSIFICATION RULES USING REGULAR ALGORITHMS}

Figure 39 presents a four-step process for mining the context sensitive associative classification rules using a traditional association algorithm and tools. Step 1 and step 2 are similar to the traditional association rule mining steps. Step 3 is for selecting rules for context sensitivity and step 4 is for selecting classification rules having class labels in the RHS only. The number of context sensitive association classification rules are very low in comparison to the number of all association rules. Figure 40 shows the comparison between the number of association rules and the number of context sensitive association 
classification rules for different support parameters; the entire CINV dataset (using all of the 6,124 records) was used.

In order to simplify the steps shown in Figure 39, we developed a novel associative classification algorithm based on the concept of a frequent pattern tree, which is able to mine the context sensitive associative classification rules in one single step. The details of the algorithm is provided in next two sections. First, we construct a context sensitive frequent associative classification tree (CS-FACT) that preserves the entire dataset in a compact frequent pattern tree. Second, the CS-FACT tree is mined to produce context sensitive associative classification rules in one single step without generating the candidate itemsets (like Apriori) or generating the all frequent patterns (like Apriori or FP-Growth).
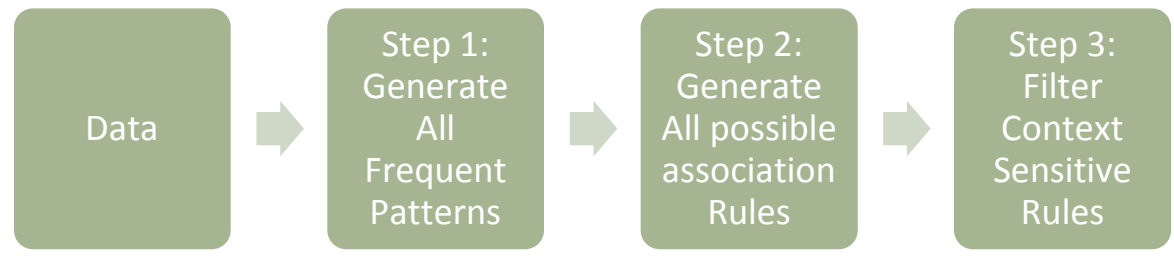

Step 4: Filter

Associative

Classification

Rules

\section{Figure 39: Steps for Mining Context Sensitive Associative Classification Rule using Regular Algorithms}




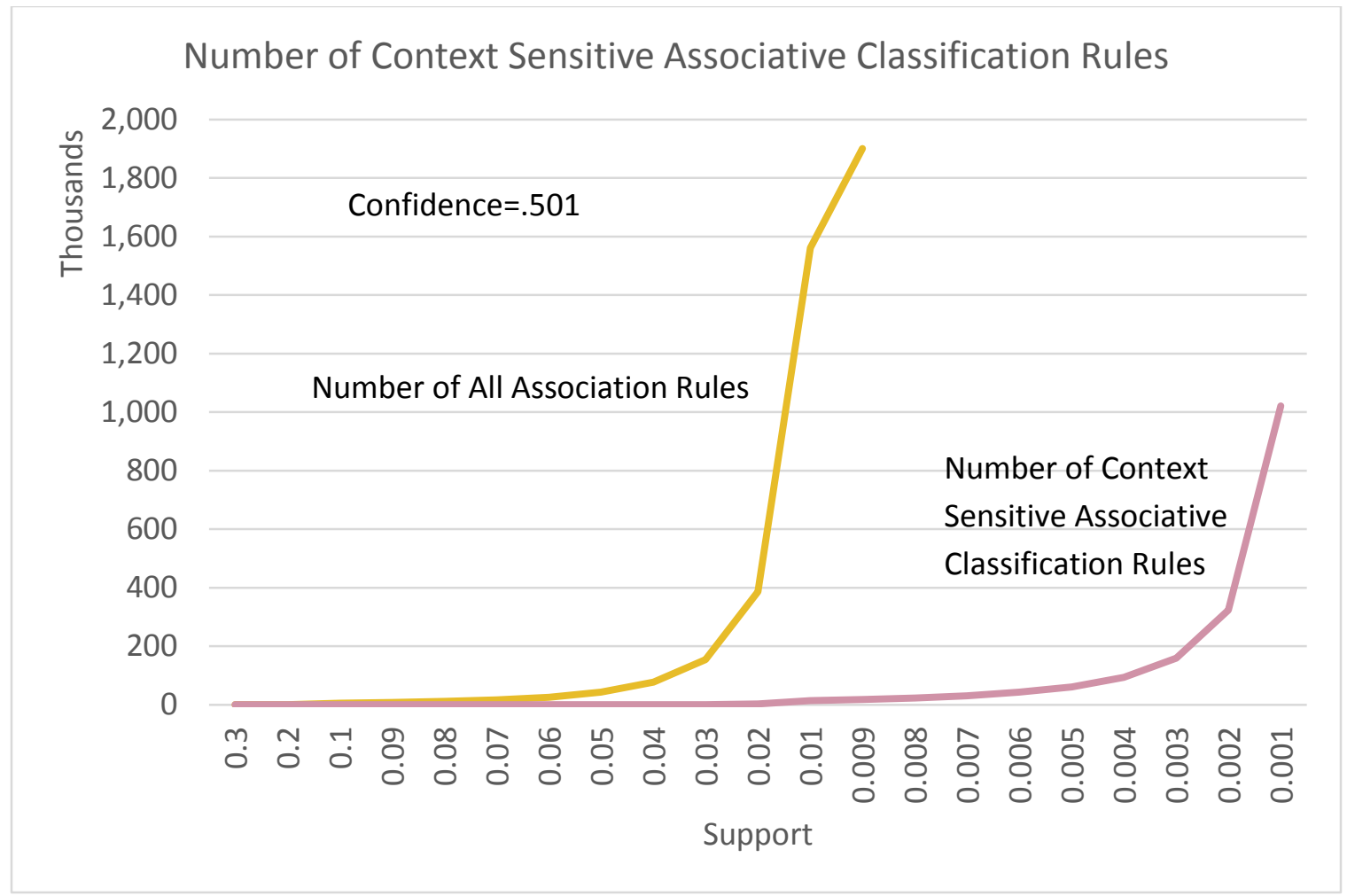

Figure 40: Number of Context Sensitive Associative Classification Rules

\subsection{CONTEXT SENSITIVE FREQUENT ASSOCIATIVE CLASSIFICATION TREE (CS-FACT)}

The entire database is converted to a compressed tree structure preserving the association information of the itemsets with respect to contexts and classes. We name the tree as "context sensitive frequent associative classification tree" (CS-FACT). The tree is mined in a divide-and-conquer manner using a bottom-up approach. A conditional database is generated from the tree for each prefix pattern and a conditional CS-FACT tree is generated for each conditional sub-database. This helps the algorithm to mine the tree in a recursive manner in order to grow the prefix patterns and reduce the size of sub-databases in each recursion. Due to the bottom-up mining approach, the tree is generated such a way so that the items are inserted at the upper nodes, and the contexts are inserted at the lower 
nodes followed by inserting the classes at the leaf nodes. This ordering mechanism is the key for direct mining of context sensitive associative classification rules as the algorithm can explore the sub-databases related to classes and contexts as prefix-patterns. This reduces the exploration of unnecessary areas of the database significantly. Algorithm 1 presents the construction process of a CS-FACT tree. The algorithm for mining the CSFACT tree is described in the next section.

Figure 41(a) presents an example transaction database in order to illustrate the Algorithm 1. First, the transaction database is scanned in order to generate a header table; the header table contains the list of contexts, items and classes, and their support counts. The header table is sorted in support count descending order of items followed by contexts and classes, consecutively. Figure 41(b) presents the ordering of the items in the header table and Figure 41(c) presents the ordering of the transactions as the order of items in the header table. In this example database, I stands for an item, CT stands for a context and C stands for a class.

Second, the root node of a CS-FACT tree is created. The database is scanned for the second time in order to insert the transactions in the tree. A branch is created for each transaction. A transaction is sorted according to the order of the items in the header table. For example, the first transaction $\{\mathrm{CT} 2 ; \mathrm{I} 1 ; \mathrm{I} 2 ; \mathrm{C} 1\}$ is sorted as $\{\mathrm{I} 2 ; \mathrm{I} 1 ; \mathrm{CT} 2 ; \mathrm{C} 1\}$ (Figure 41(c)) and a branch is created where I 2 is linked to root, I1 is linked to I2, CT2 is linked to I1, and C1 is linked to CT2 (Figure 42(a)). The count of each node is set to 1 . The second transaction $\{\mathrm{I} 2 ; \mathrm{CT} 1 ; \mathrm{C} 1\}$ would result in a branch where $\mathrm{I} 2$ is linked to root, $\mathrm{CT} 1$ is linked to $\mathrm{I} 2$ and $\mathrm{C} 1$ is linked to $\mathrm{CT} 1$. This branch will share the $\mathrm{I} 2$ node as a common prefix with the first branch. Therefore, the count of 12 node is incremented to 2 (Figure 42 (b)). The 
count of the common prefix nodes are incremented by 1 every time a branch is generated.

Figure 42(a), Figure 42 (b), Figure 43, Figure 44, Figure 45, Figure 46, Figure 47, Figure 48, and Figure 49 presents the steps of inserting the transactions 1 through 9 in a CS-FACT tree. The insertion path is indicated with red arrows.

\section{Algorithm 1: Construction of Context Sensitive Frequent Associative Classification Tree}

Input:

- D, a transaction database

- List of contexts

- List of classes

procedure CS_FACT(D, contexts, classes)

(1) Scan the transaction database D and create a set headerTable, containing the list of contexts, items and classes, and their support count.

(2) Order headerTable in support count descending order of items followed by contexts and classes, consecutively.

(3) Create the root node of a CS-FACT tree named csFactTree and label the node as root.

(4) For each transaction $T$ in $D$ do the following:

a. Order the items, contexts and classes in $\mathrm{T}$ according to the order of headerTable.

b. Set currentNode to root.

c. For each element in $\mathrm{T}$ do the following

i. Call insertElementToCSFACT(element, 1, currentNode); this procedure returns a childNode.

ii. set currentNode to childNode

(5) Return the csFactTree.

procedure insertElementToCSFACT(element, countOfElement, currentNode)

(1) If currentNode has a childNode such that childNode.elementName = elment.name, then do the following:

a. increment childNode.count by countOfElement.

b. Return childNode

(2) Else create a new node called childNode and do the following:
a. Set childNode.element-name to element.name
b. Set childNode.count to countOfElement
c. Set childNode.parent to currentNode
d. Link childNode to the nodeLinks where childNode.element- name $=$ nodeLinks.element-name
e. Return childNode 


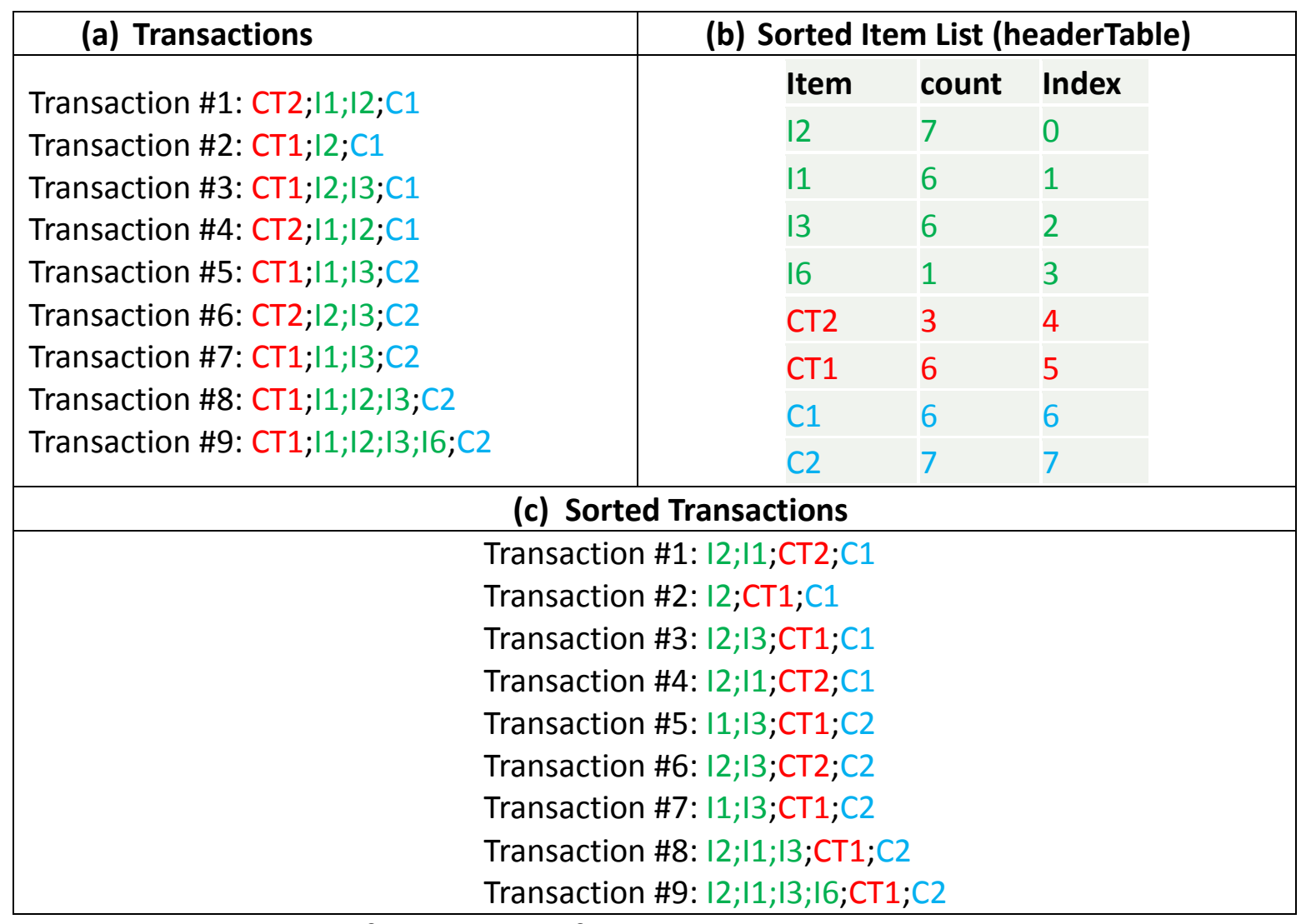

Figure 41: Example of Transactions for Generating Context Sensitive Associative Classification Tree - (a) Transaction, (b) Sorted item list, (c) Sorted Transactions 


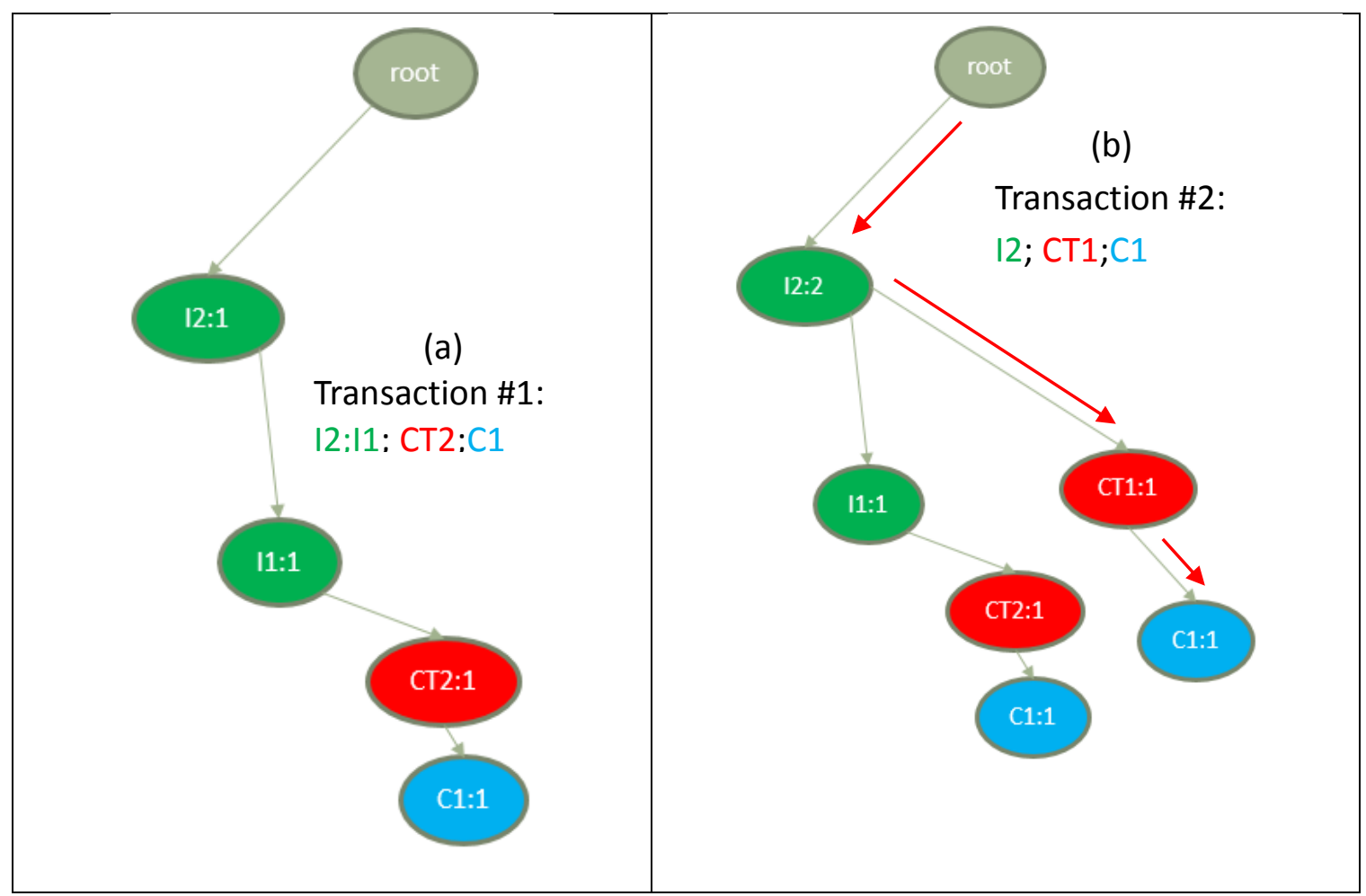

Figure 42: Construction of CS-FACT - (a) Insert Transaction \#1 and (b) Insert Transaction \#2

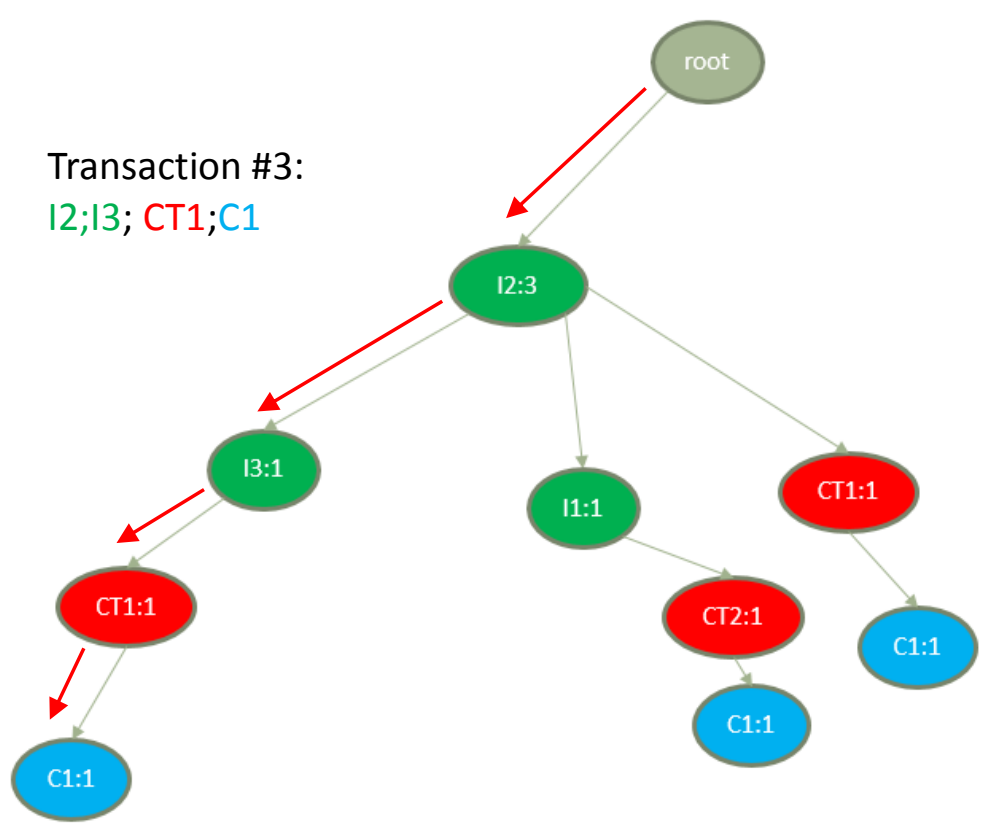

Figure 43: Construction of CS-FACT - Insert Transaction \#3 


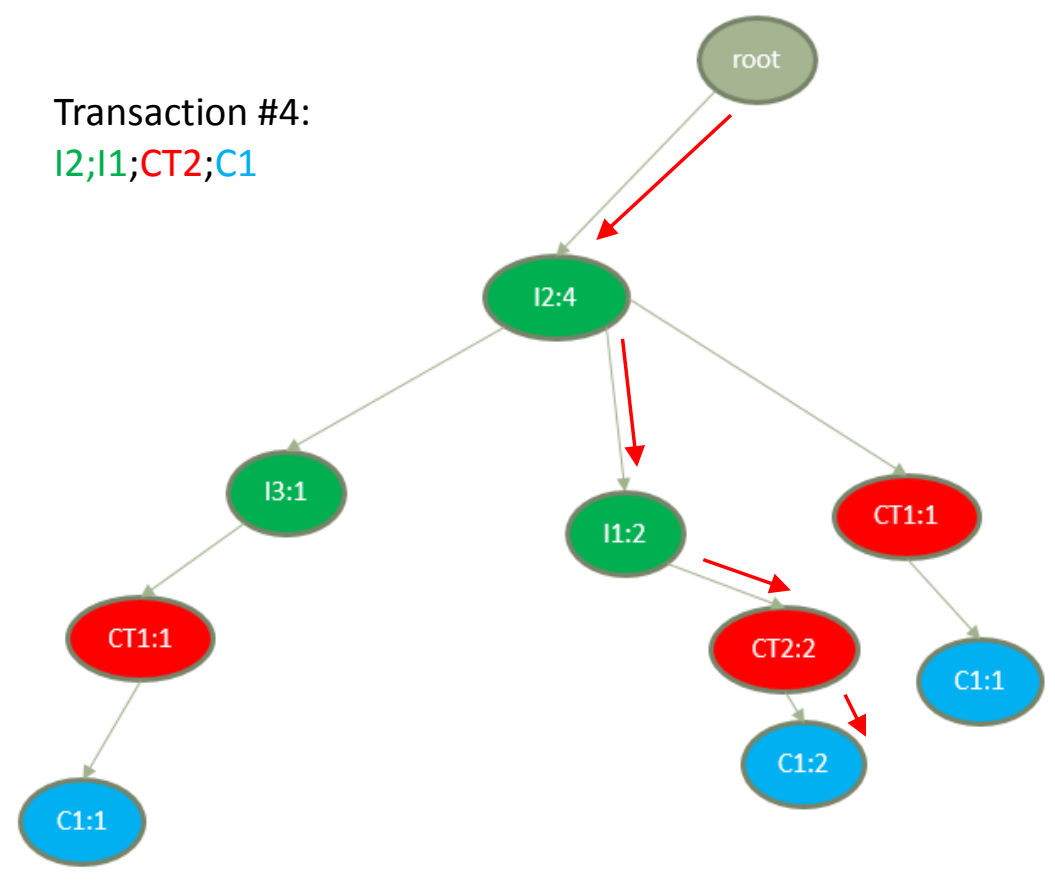

Figure 44: Construction of CS-FACT - Insert Transaction \#4

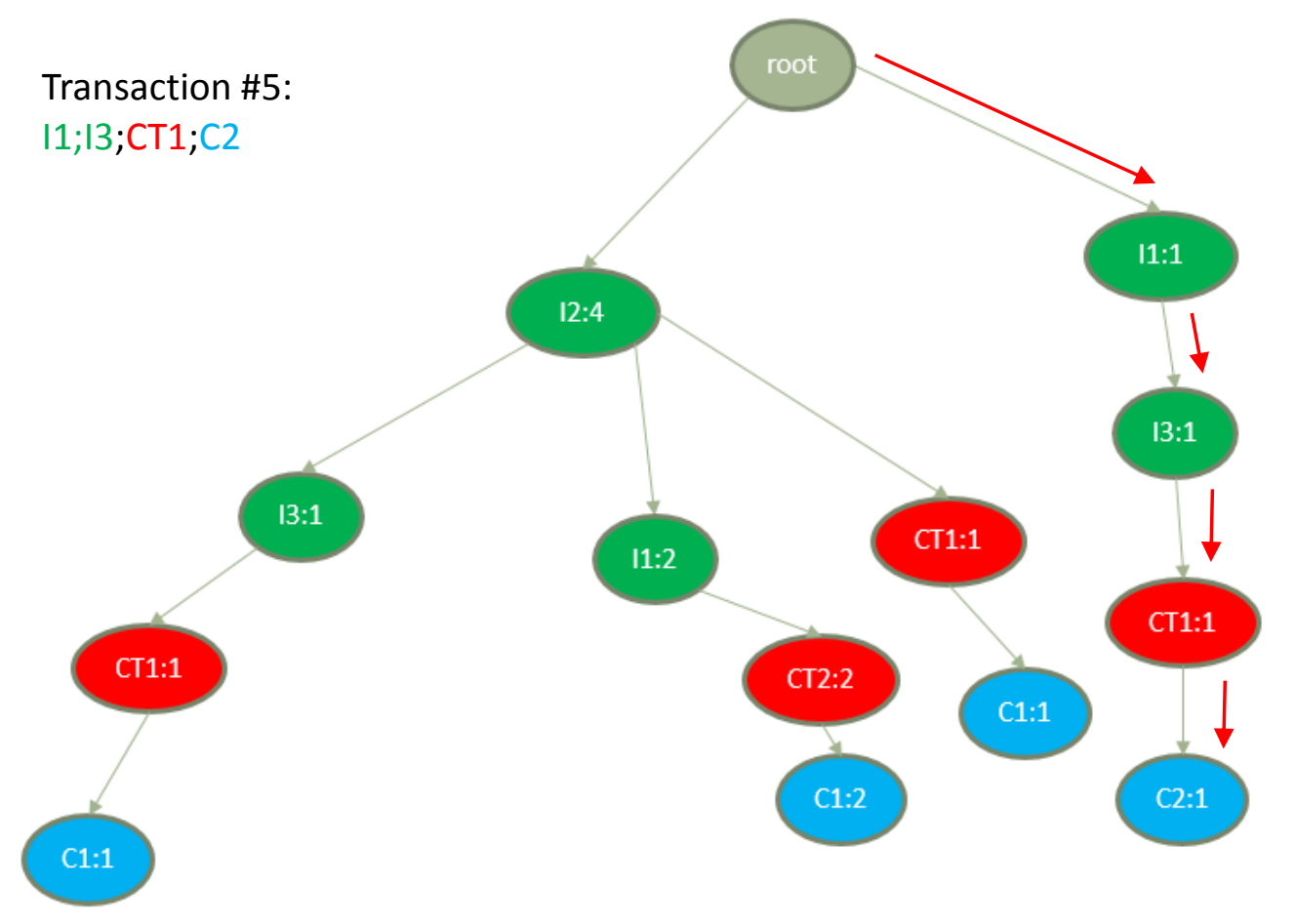

Figure 45: Construction of CS-FACT - Insert Transaction \#5 


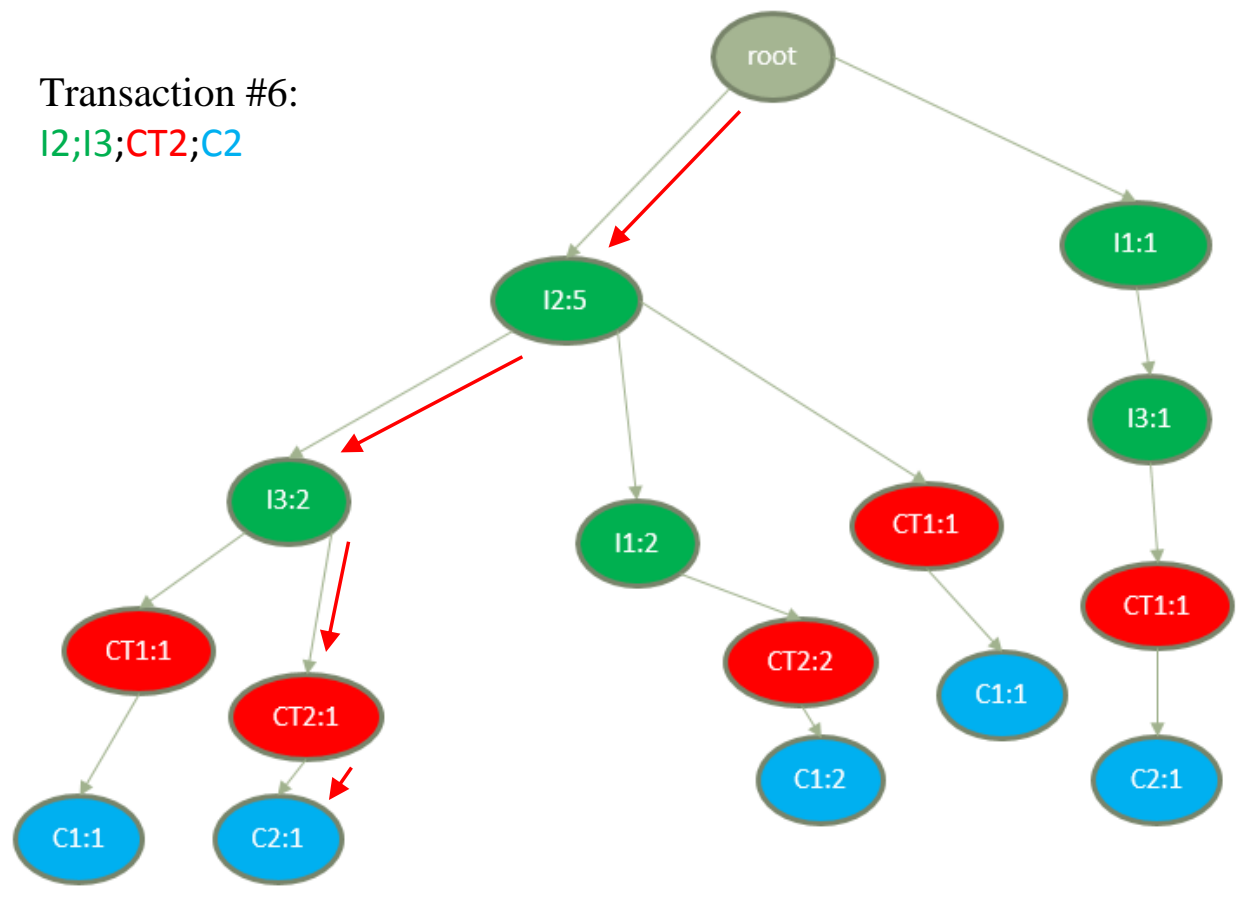

Figure 46: Construction of CS-FACT - Insert Transaction \#6

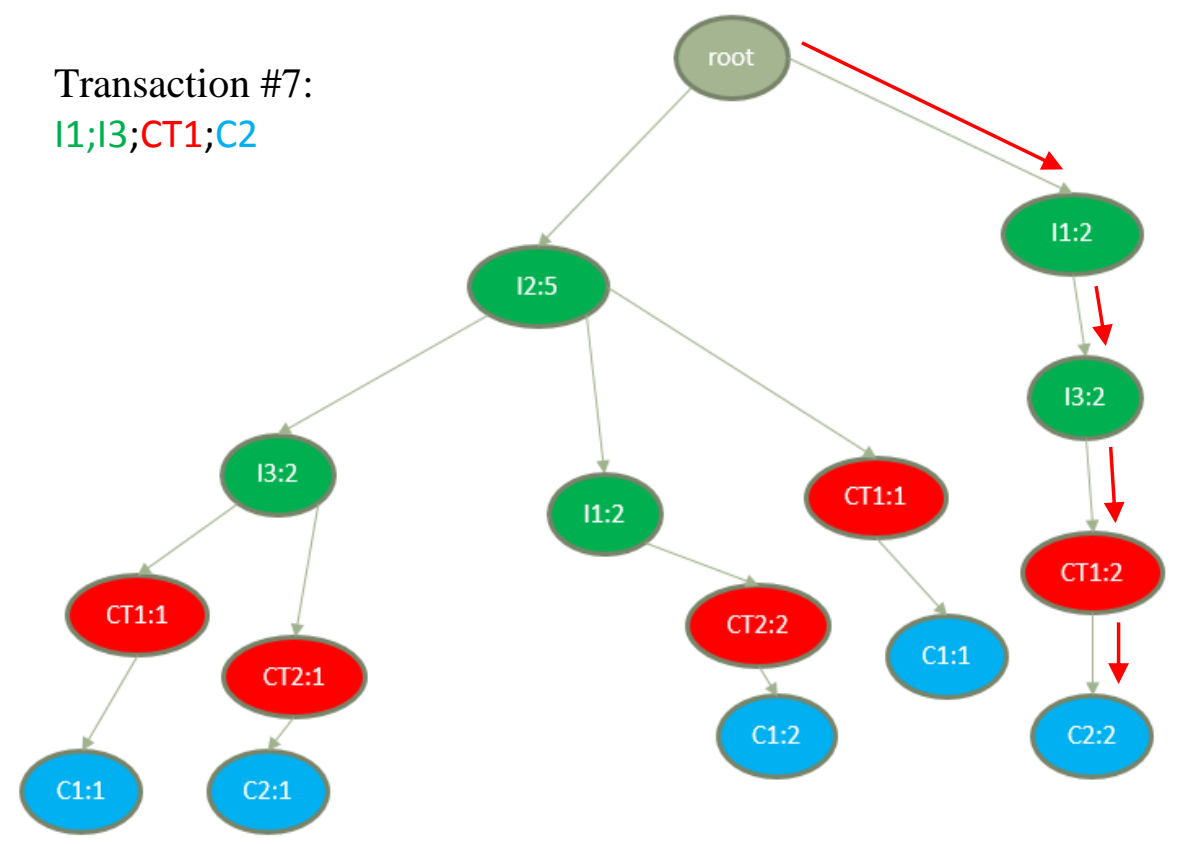

Figure 47: Construction of CS-FACT - Insert Transaction \#7 


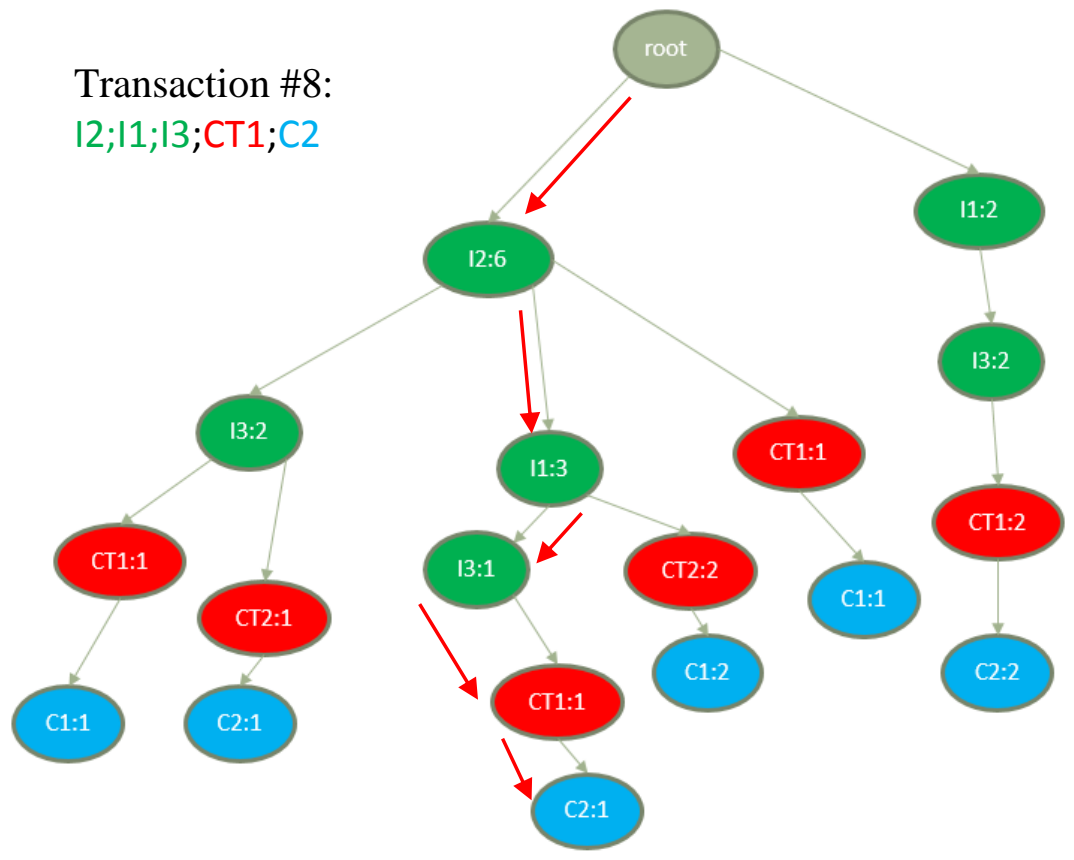

Figure 48: Construction of CS-FACT - Insert Transaction \#8s

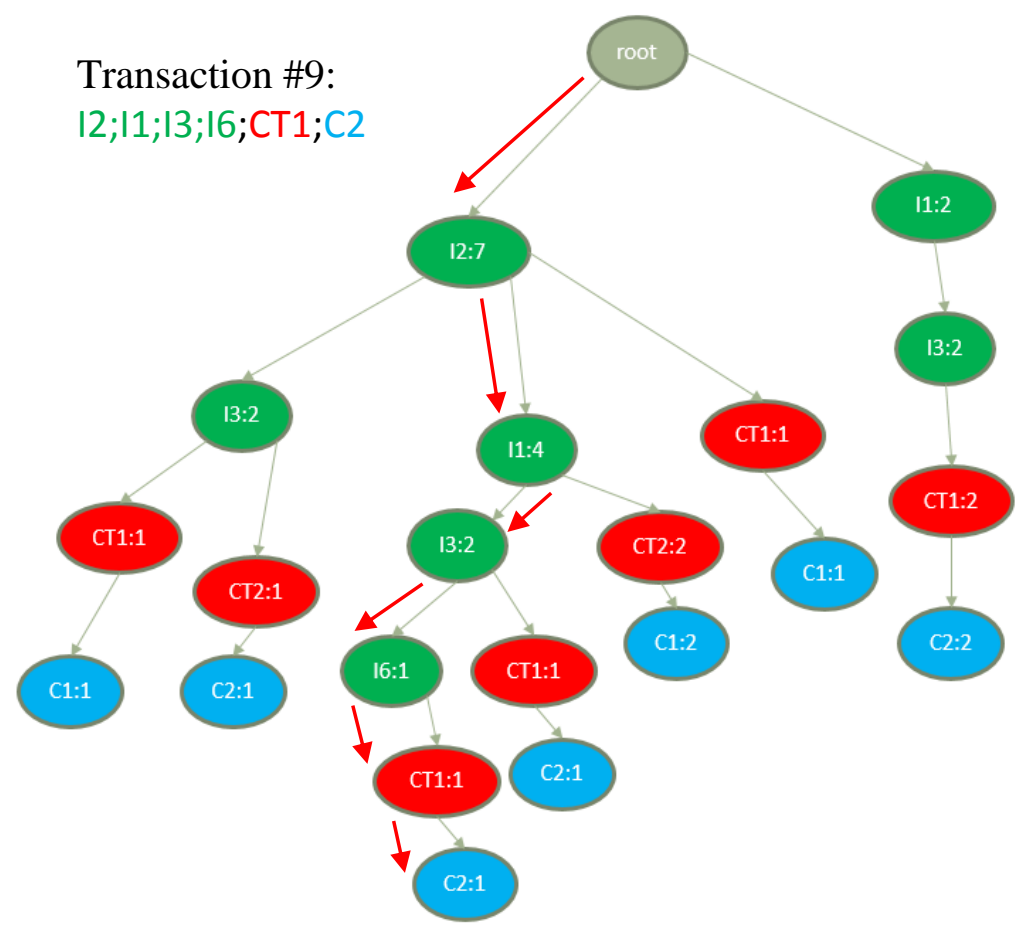

Figure 49: Construction of CS-FACT - Insert Transaction \#9 


\subsection{MINING CONTEXT SENSITIVE ASSOCIATIVE CLASSIFICATION RULE USING CS-FACT}

Algorithm 2 presents the detailed process for mining a CS-FACT tree for producing context sensitive associative classification rules. In the first iteration, the algorithm starts with a class as a prefix-pattern, and generates a conditional sub-database and CS-FACT tree for this class. The sub-database is also called as conditional pattern base. For generating a conditional pattern base for this class, all the node-links of the class are listed first. For each node-link of this class, a path from the parent of the class-node to the root of the tree is generated; the items in this path form a transaction, which becomes a part of the conditional pattern base for this class. The count of all the items in this transaction is set to the support count of the class-node. For example, the conditional pattern base for the class $\mathrm{C} 1$ is $\{\mathrm{CT} 2 ; \mathrm{I} 1 ; \mathrm{I} 2: 2\},\{\mathrm{CT} 1 ; \mathrm{I} 2: 1\}$, and $\{\mathrm{CT} 1 ; \mathrm{I} 2 ; \mathrm{I} 3: 1\}$ (Figure 50); here, the prefixpattern is $\{\mathrm{C} 1\}$. This conditional pattern based is used for generating a conditional CSFACT tree (Algorithm 1). Figure 51 presents the conditional frequent pattern tree generated from the conditional pattern base for the class $\mathrm{C} 1$. This tree represents the conditional pattern base for transactions containing $\mathrm{C} 1$ and does not contain any class.

In the second iteration, a new prefix pattern is generated for each context by appending the context to a class prefix-pattern, and a conditional pattern base and a CSFACT tree is generated for this prefix-pattern. At this point, the prefix-pattern contains classes and contexts, and the conditional CS-FACT trees contains only the items. For example, a prefix-pattern is generated for $\mathrm{C} 1$ by appending the context $\mathrm{CT} 2$ as $\{\mathrm{CT} 2$, $\mathrm{C} 1: 2\}$; see the procedure generatePrefixPattern in Algorithm 2. Then, a conditional CSFACT tree is generated for this prefix-pattern (see Figure 52 and Figure 53). 
In the subsequent iterations, a prefix-pattern is grown by appending the items from the conditional CS-FACT tree; see the procedure mine_CS_ACR in Algorithm 2. This is a recursive procedure. A conditional CS-FACT tree is further mined if the corresponding prefix-pattern contains a class item and a context item. This step is very useful because unnecessary rules are not generated. Every time a prefix-pattern is generated, the pattern is tested for eligibility (the prefix pattern contains a class item and a context item); a context sensitive associative classification rule is generated immediately for an eligible prefixpattern. Figure 54 presents all the context sensitive associative classification rules generated from the transaction database in Figure 41(a).

Algorithm 2 generates the support count for the entire rule only but not for the antecedent (LHS) or consequent (RHS); thus calculations of confidence and lift are not possible. At the same time, we do not want to scan the entire database to generate support count for those itemsets because the database scan is an expensive process. We developed another algorithm to generate support count for any itemset from a CS-FACT tree; thus additional database scan is not required. Algorithm 3 illustrates the detailed steps. First, the itemset is ordered according to the order of the items in the header table. For the last element in the ordered itemset, all the node-links are listed from the CS-FACT tree. Second, for a node in the node-links, a path from the node to the root is generated. A pattern is generated for this path if the path contains all the items in the itemset. The support count of the pattern is set to the least support count of the elements in the itemset. Finally, the support count of the itemset is calculated by taking the summation of the support count of all the patterns. Figure 55 presents the steps for generating the support count for the itemset $\{$ I1, I3\}. Following this method, we also pre-compute all the support count for each 
contexts and the support count for each context and class combinations; these are used for calculating the context sensitive measures as explained in section 5.8. 


\section{Algorithm 2: Mining Context Sensitive Associative Classification Rule using CS-FACT}

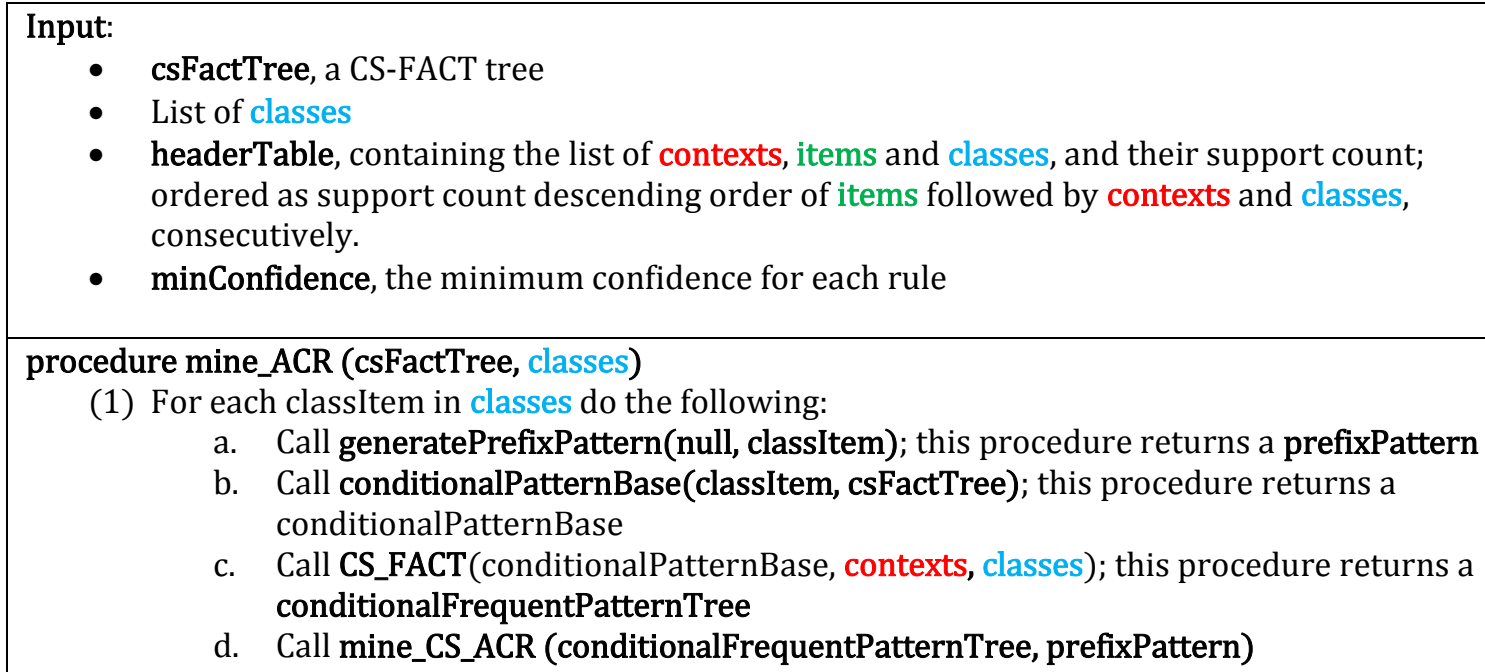

- List of classes

- $\quad$ headerTable, containing the list of contexts, items and classes, and their support count; ordered as support count descending order of items followed by contexts and classes, consecutively.

- $\quad$ minConfidence, the minimum confidence for each rule

procedure mine_ACR (csFactTree, classes)

(1) For each classItem in classes do the following:

a. Call generatePrefixPattern(null, classItem); this procedure returns a prefixPattern

b. Call conditionalPatternBase(classItem, csFactTree); this procedure returns a conditionalPatternBase

c. Call CS_FACT(conditionalPatternBase, contexts, classes); this procedure returns a conditionalFrequentPatternTree

d. Call mine_CS_ACR (conditionalFrequentPatternTree, prefixPattern)

procedure mine_CS_ACR (conditionalFrequentPatternTree, prefixPattern)

(1) For each element in the headerTable of conditionalFrequentPatternTree do the following:

a. Call generatePrefixPattern(prefixPattern, element); this procedure returns a newPrefixPattern

b. If the newPrefixPattern does not contain a class and/or a context then do nothing

c. Else do the following:

i. Generate a contextSensitiveAssociativeClassificationRule from the conditionalFrequentPatternTree

1. If contextSensitiveAssociativeClassificationRule.confidence $>=$ minConfidence then keep the rule

ii. Call conditionalPatternBase(element, conditionalFrequentPatternTree); this procedure returns a conditionalPatternBase

iii. Call CS_FACT(conditionalPatternBase, contexts, classes); this procedure returns a newConditionalFrequentPatternTree

iv. If newConditionalFrequentPatternTree is not empty then call this procedure recursively as mine_CS_ACR(newConditionalFrequentPatternTree, newPrefixPattern)

procedure generatePrefixPattern (prefixPattern, element)

(1) Append element to the prefixPattern

(2) Set support count of prefixPatter to element.count

(3) Return prefixPattern

procedure conditionalPatternBase(element, csFACTree)

(1) Find all nodeLinks of the element from the csFactTree

(2) Create an empty conditionalPatternBase

(3) For each node in nodeLinks do the following:

a. Generate a set of items named pathToRootItemset containing all the elements in the path from node.parent to root

b. Set support count for pathToRootItemset as node.support_count.

c. Add pathToRootItemset to conditionalPatternBase

(4) Return conditionalPatternBase 


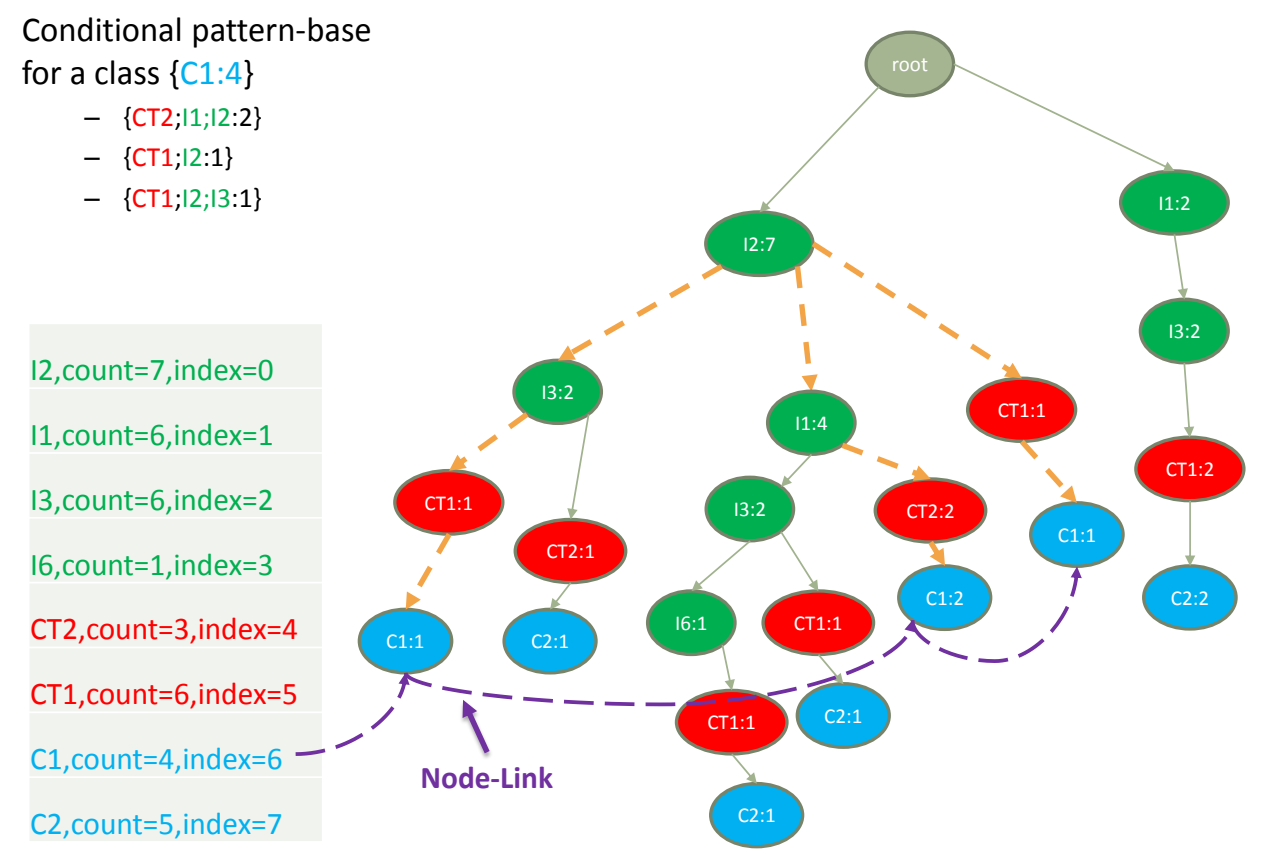

Figure 50: Generate a conditional pattern-base 


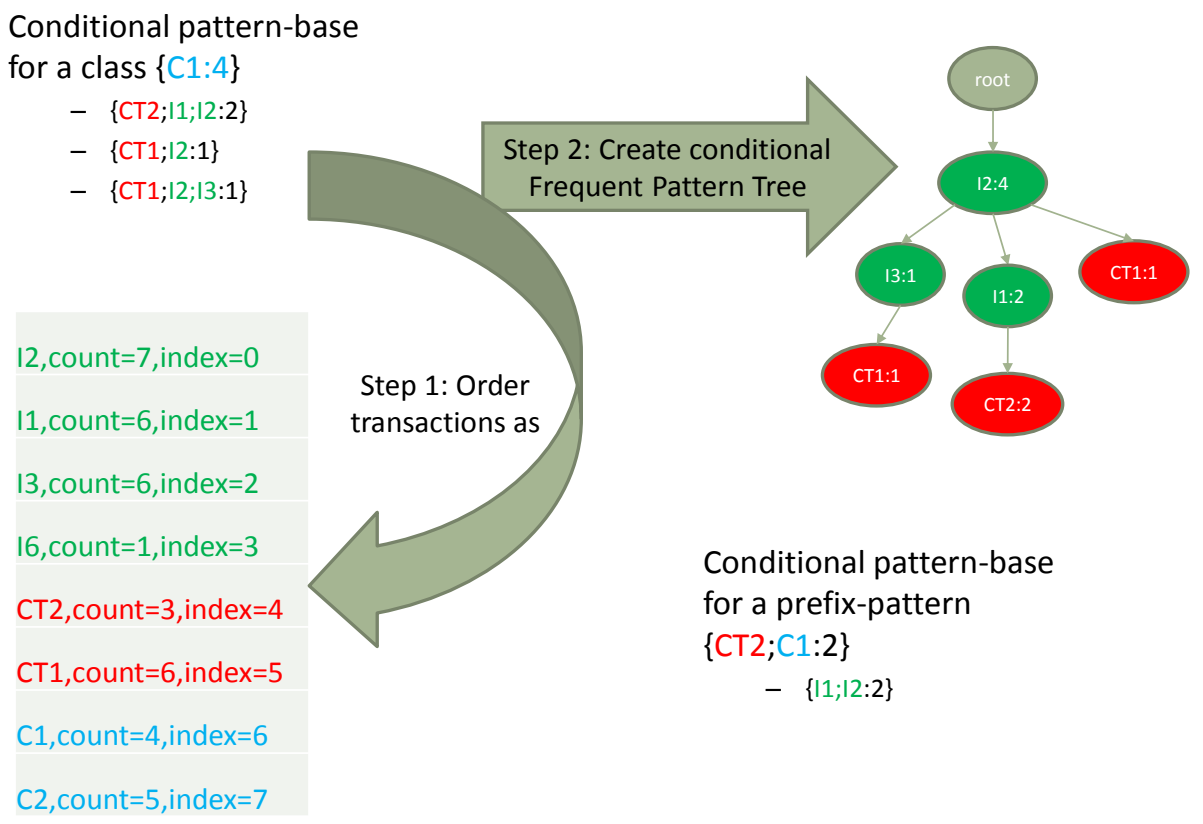

\section{Figure 51: Conditional Frequent Pattern Tree for a Class}

Conditional pattern-base for a prefix-pattern \{CT2; 1 1:2\}

$$
-\{\mid 1 ; 12: 2\}
$$

\begin{tabular}{|c|}
\hline 12 , count $=7$, index $=0$ \\
\hline 11 , count=6, index $=1$ \\
\hline 13 , count $=6$, index $=2$ \\
\hline 16 , count=1, index $=3$ \\
\hline$C T 2$, count $=3$, index $=4$ \\
\hline CT1, count $=6$, index $=5$ \\
\hline C1, count $=4$, index $=6$ \\
\hline
\end{tabular}

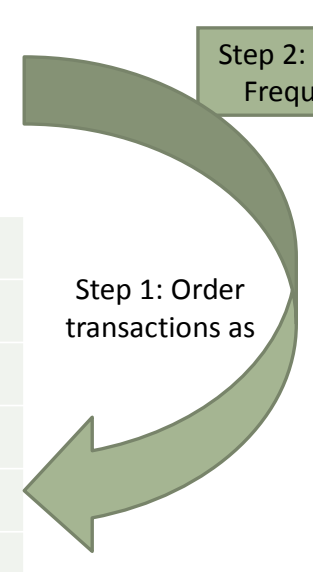

Context Sensitive Associative Classification Rule

$$
\begin{aligned}
& \text { - }\{\mathrm{CT} 2\}\{11\} \rightarrow\{\mathrm{C} 1\} \\
& \text { - Support count }=2
\end{aligned}
$$

Figure 52: Prefix Pattern and Association Rule 


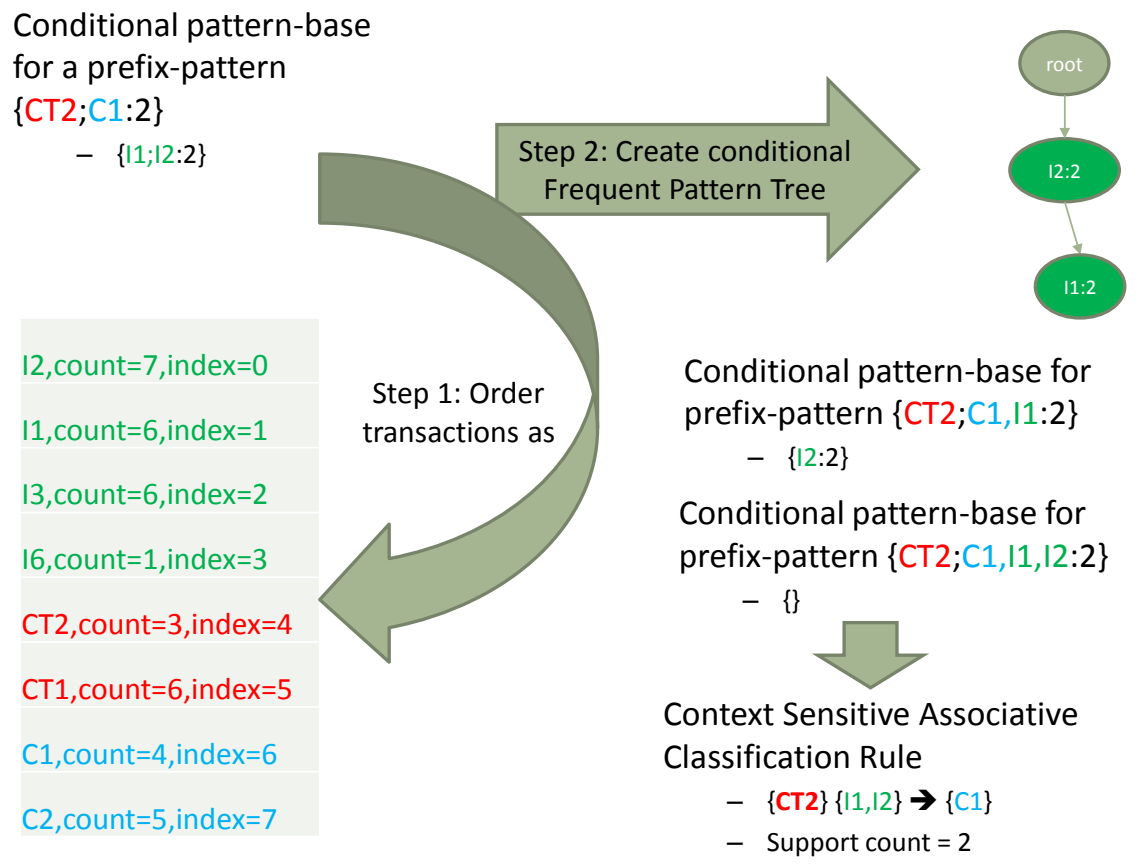

Figure 53: Conditional Frequent Pattern Tree for a Prefix Pattern

$[[\{C T 2\}: 3 ;\{\mid 1\}]: 2 \rightarrow C 1]: 2$
$[[\{C T 2\}: 3 ;\{\mid 2\}]: 3 \rightarrow C 1]: 2$
$[[\{C T 2\}: 3 ;\{|2 ;| 1\}]: 2 \rightarrow C 1]: 2$
$[[\{C T 1\}: 6 ;\{\mid 1\}]: 4 \rightarrow C 2]: 4$
$[[\{C T 1\}: 6 ;\{\mid 3\}]: 5 \rightarrow C 2]: 4$
$[[\{C T 1\}: 6 ;\{|1 ;| 1\}\}]: 4 \rightarrow C 2]: 4$
$[[\{C T 1\}: 6 ;\{\mid 2 ; 11\}]: 2 \rightarrow C 2]: 2$
$[[\{C T 1\}: 6 ;\{|2 ;| 3\}]: 3 \rightarrow C 2]: 2$
$[[\{C T 1\}: 6 ;\{|2 ; 11 ;| 3\}]: 2 \rightarrow C 2]: 2$

Figure 54: Context Sensitive Associative Classification Rules 


\section{Algorithm 3: Mining Support Count for an Itemset using a CS-FACT Tree}

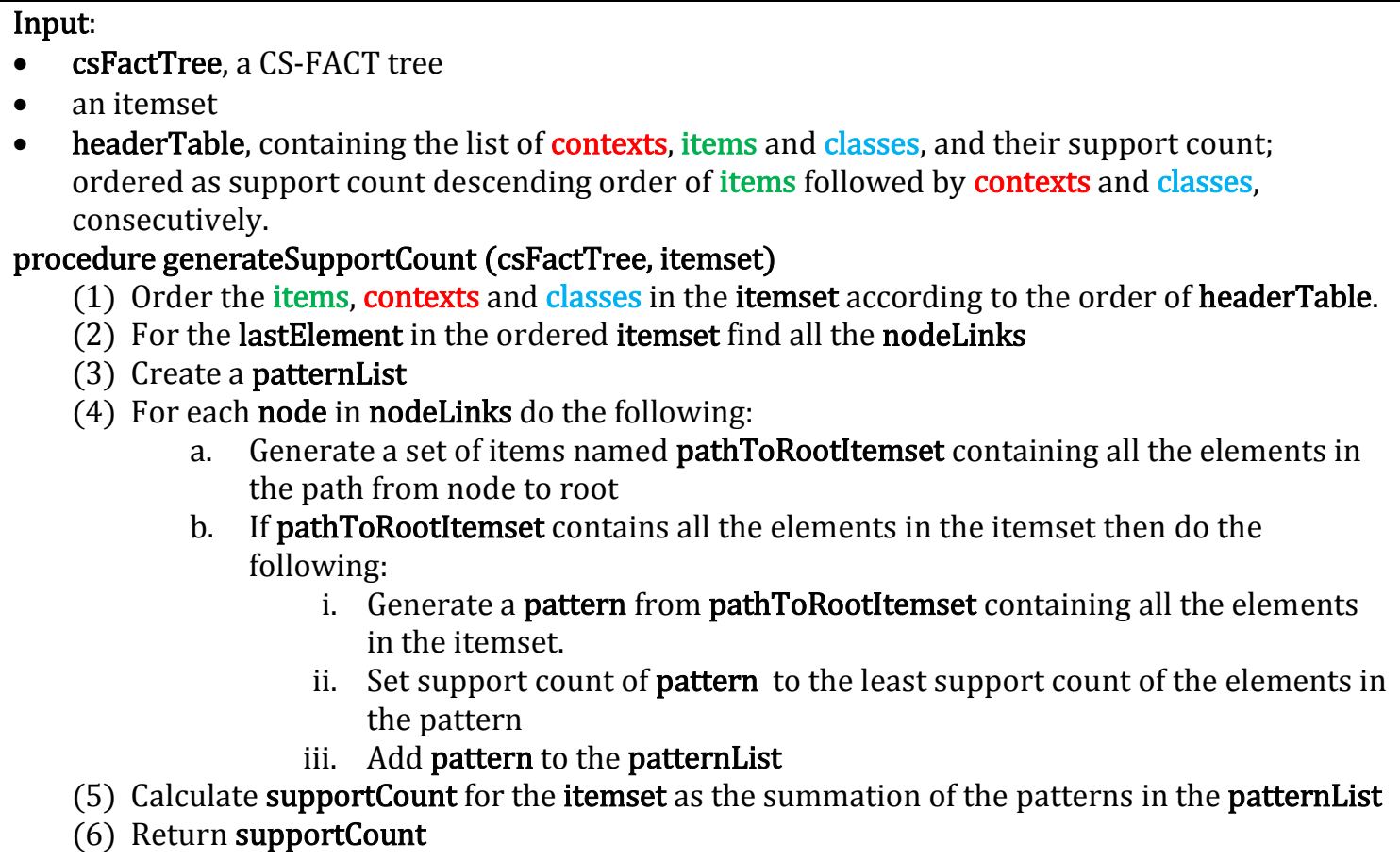

(1) Order the items, contexts and classes in the itemset according to the order of headerTable.

(2) For the lastElement in the ordered itemset find all the nodeLinks

(3) Create a patternList

(4) For each node in nodeLinks do the following:

a. Generate a set of items named pathToRootItemset containing all the elements in the path from node to root

b. If pathToRootItemset contains all the elements in the itemset then do the following:

i. Generate a pattern from pathToRootItemset containing all the elements in the itemset.

ii. Set support count of pattern to the least support count of the elements in the pattern

iii. Add pattern to the patternList

(5) Calculate supportCount for the itemset as the summation of the patterns in the patternList

(6) Return supportCount 


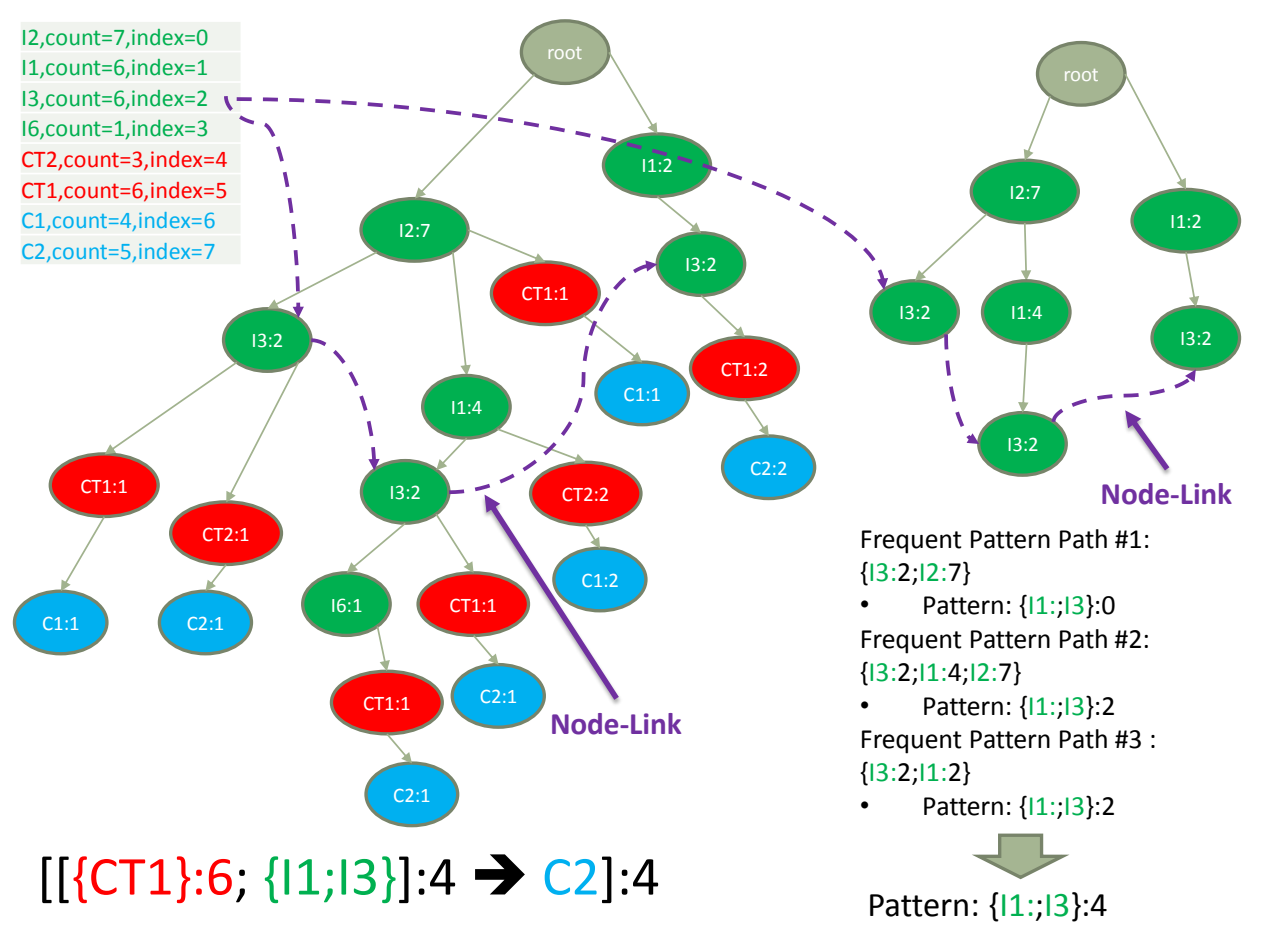

Figure 55: Mining Support Count for Antecedent

As discussed in the section 5.9 that mining context sensitive association classification rules using regular association rule mining algorithms is a multi-step process. On the other hand, our algorithm generates the rules in one single step (Figure 56). The CS-FACT does not require the generation of intermediate candidate itemsets (like in the Apriori algorithm) or generating the all possible frequent itemsets (like in the Apriori or FP-Growth algorithm). Thus, our method requires very lower processing time than other algorithms (Figure 57).

\section{Data}

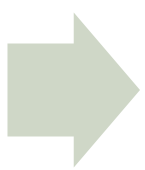

\section{Context Sensitive Associative Classification Rules}

Figure 56: Steps for Mining Context Sensitive Associative Classification Rule using CSFACT 


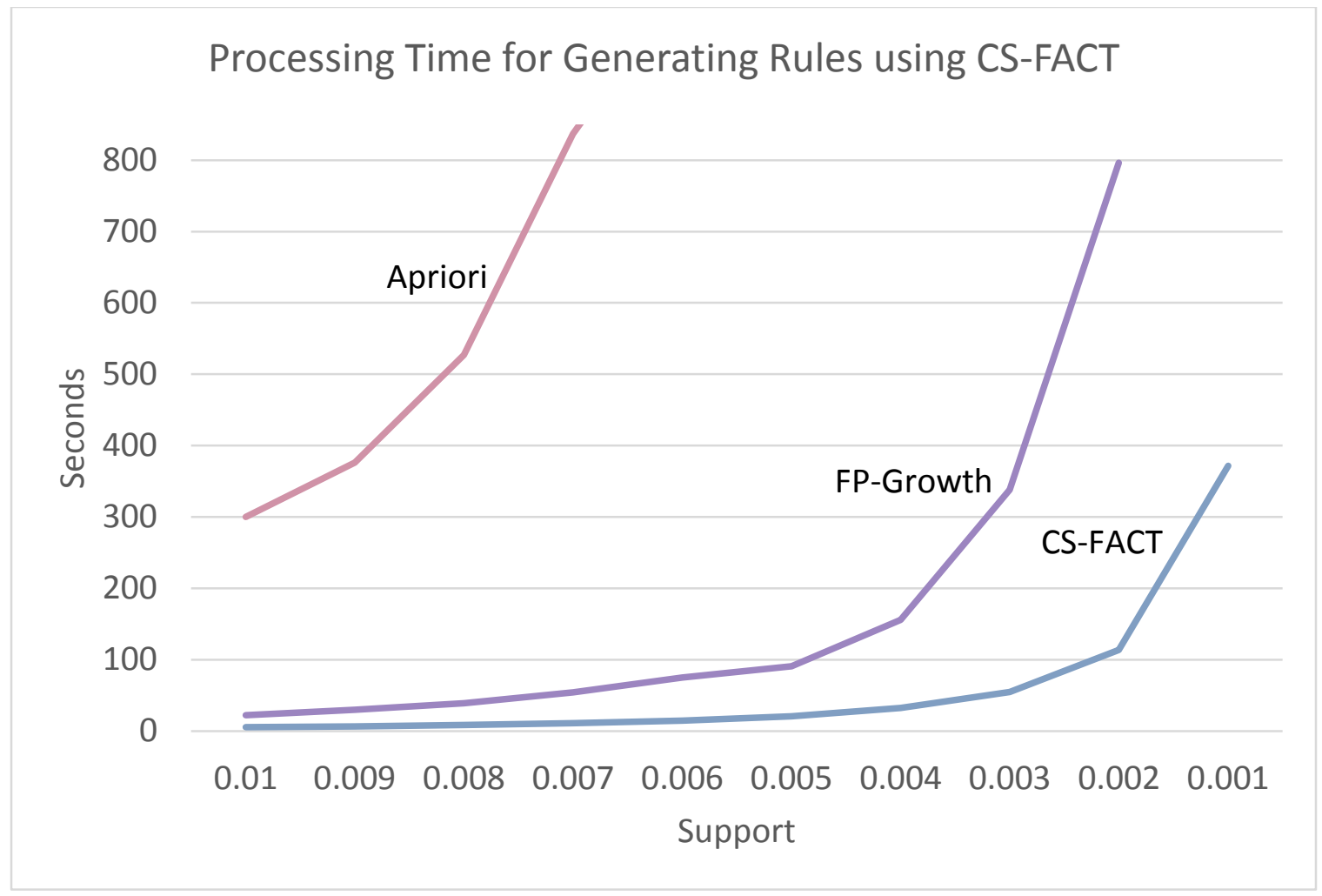

Figure 57: Processing Time for Generating Context Sensitive Associative Classification Rules using CS-FACT 


\section{CHAPTER 6 : THE PREDICTION ENGINE}

\subsection{A DYNAMIC APPROACH}

We have developed the prediction engine based on a dynamic approach (see Figure 58). The training phase consists of generating context sensitive associative classification rules and pruning the unnecessary rules. The dynamic prediction phase involves extracting all qualified associative classification rules for a prediction query within a context in realtime and generating a dynamic prediction recommendation based on those rules.

\begin{tabular}{|c|}
\hline Training Steps \\
\hline - Generate Context \\
Sensitive Associative \\
Classification Rules \\
- Prune Unnecessary \\
Rules \\
\end{tabular}

\section{Dynamic Prediction Steps}

- Extract All Supported Rules for a Prediction Query in Real-Time

- Generate Prediction Recommendation Using those Rules

Figure 58: Training and Prediction Steps 


\subsection{SYSTEM ARCHITECTURE}

Figure 59 presents the system architecture for the prediction engine and Figure 60 presents the flow diagram of the system. A prediction process first starts with a prediction query, which is then processed by the prediction engine and passed to the rule-extractor module. The rule-extractor module passes all the qualified rules to the multi-rule predictor module, which generates a prediction recommendation. The training step is driven by the CS-FACT tree, which is a compact tree structure preserving enough information for mining necessary rules. The tree can be updated in real-time by adding a set of transactions to the tree, and the association rule database can be updated accordingly from the tree. As a result, a prediction result is always based on the up-to-date knowledge. The system components are described in the following sections. 


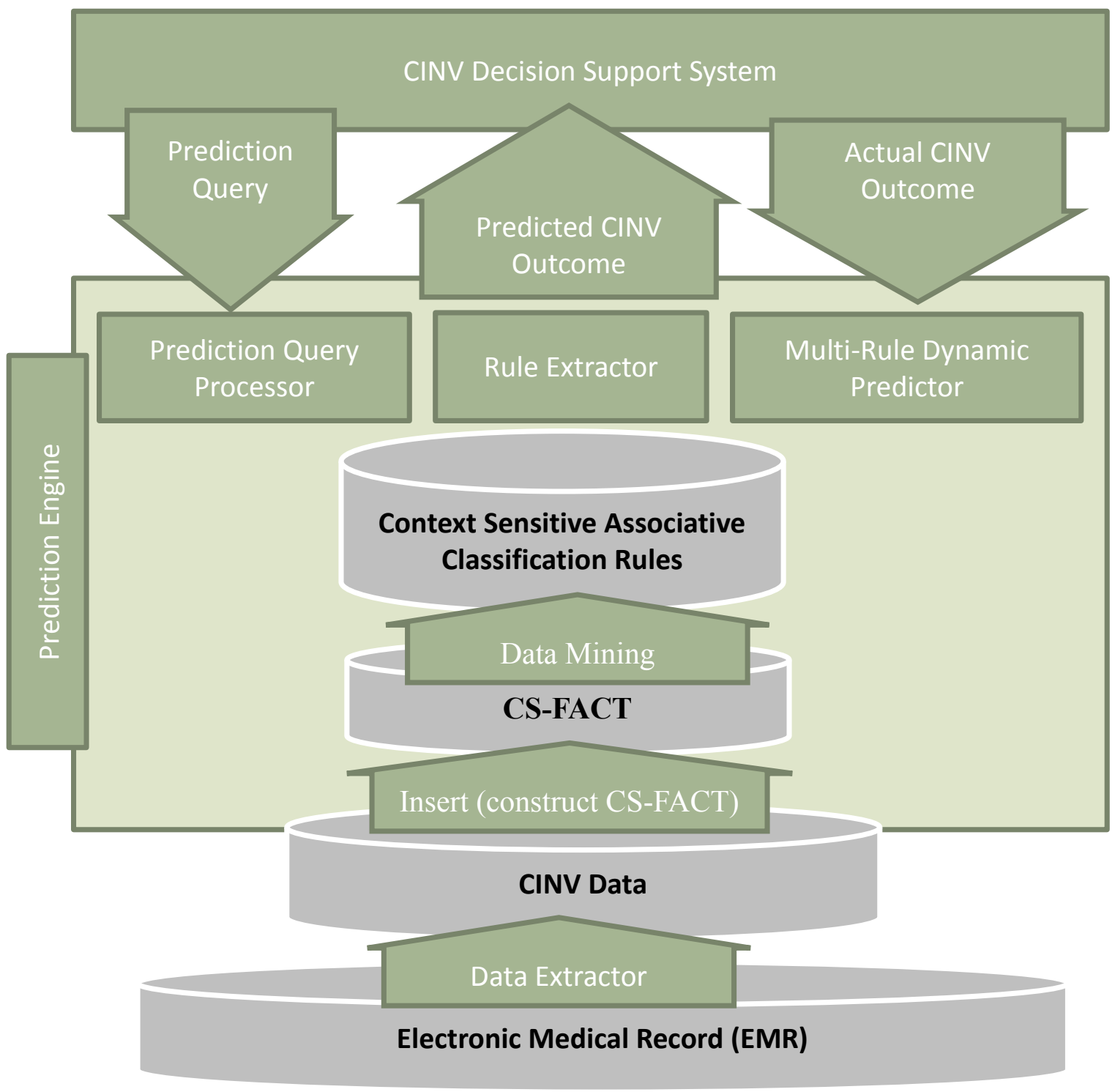

Figure 59: System Architecture 


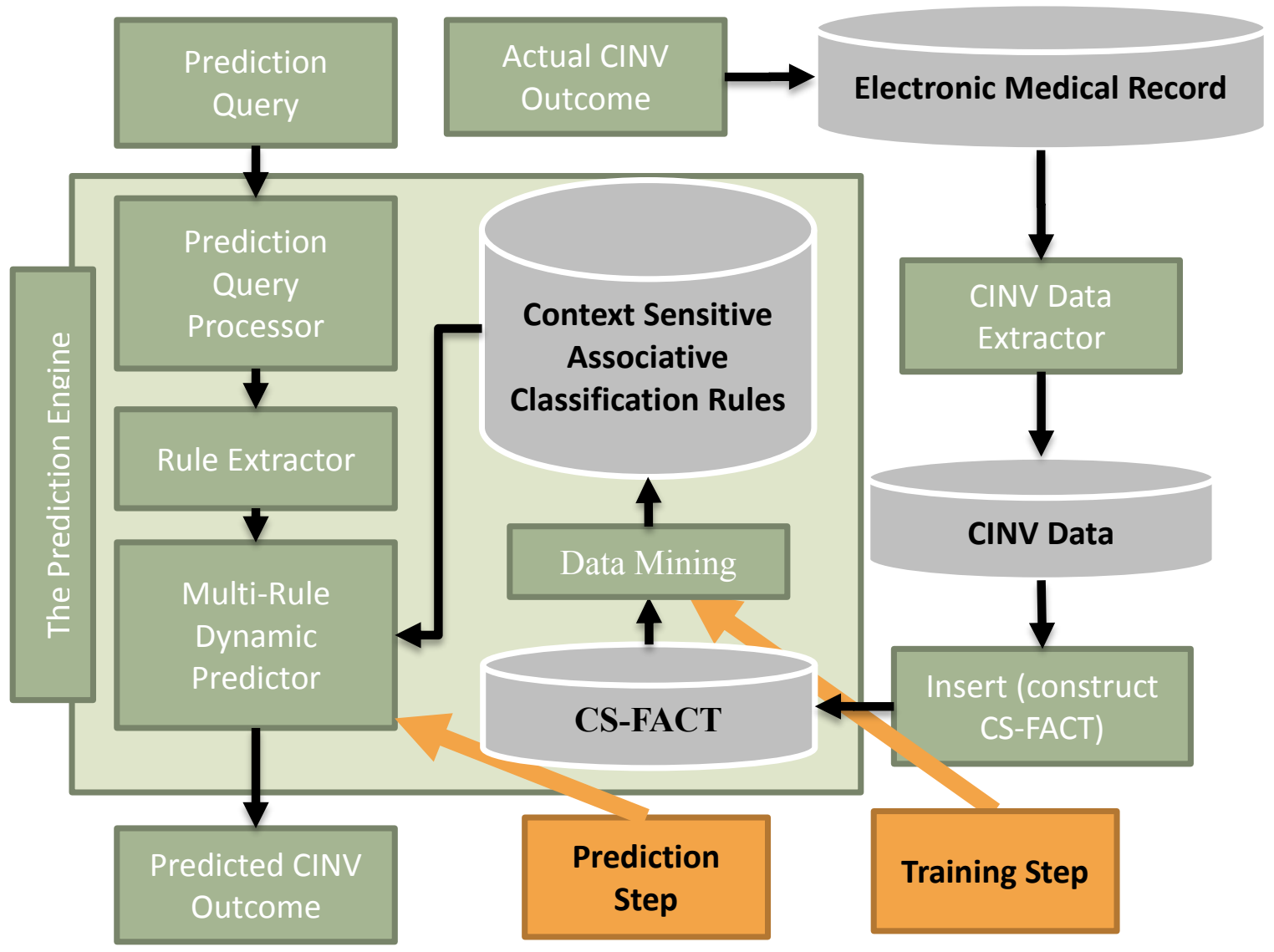

Figure 60: CINV Decision Support System Flow Diagram

\subsubsection{Prediction Query}

A prediction query is a query to the prediction engine requesting a predicted outcome for a set of attribute-value pairs from the engine (see Figure 61). The query is formatted as (Contexts) AND (Risk Factors) $=>$ (Class/Dependent variable). For example, a prediction query could be "what is the Acute CINV outcome for a patient with high emetogenic chemotherapy and the following risk factors: Dehydration=Yes AND Anxiety=Yes?", it is formatted as (CINV Phase=Acute, Emetogenecity=High) AND $($ Dehydration $=$ Yes AND Anxiety $=$ Yes $)=>(\mathrm{CINV}=? ?))$. 


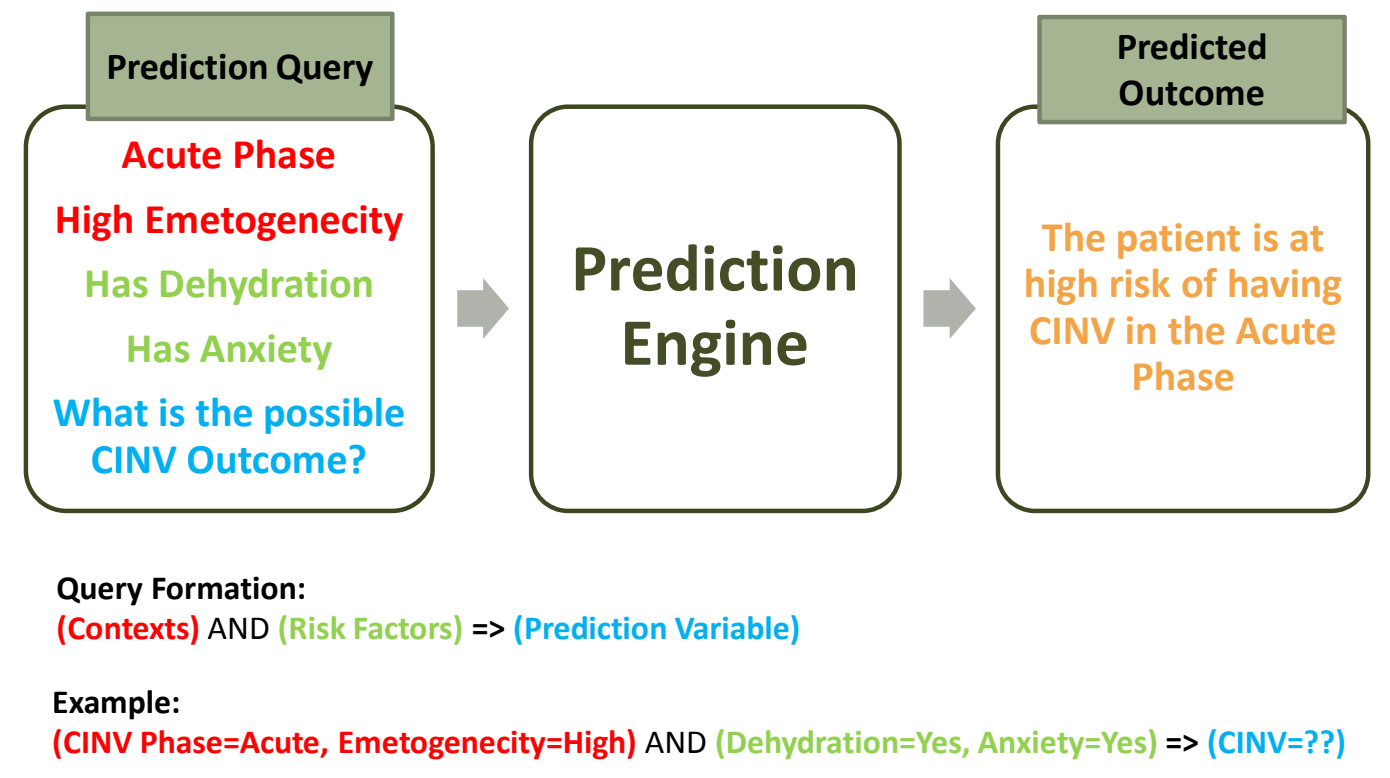

Figure 61: Prediction Query

\subsubsection{Prediction Query Processor}

The prediction query processor is designed to process a prediction query and extract the components of the query. A prediction query has three components: contexts, risk factors and class/dependent variable (Figure 62). 


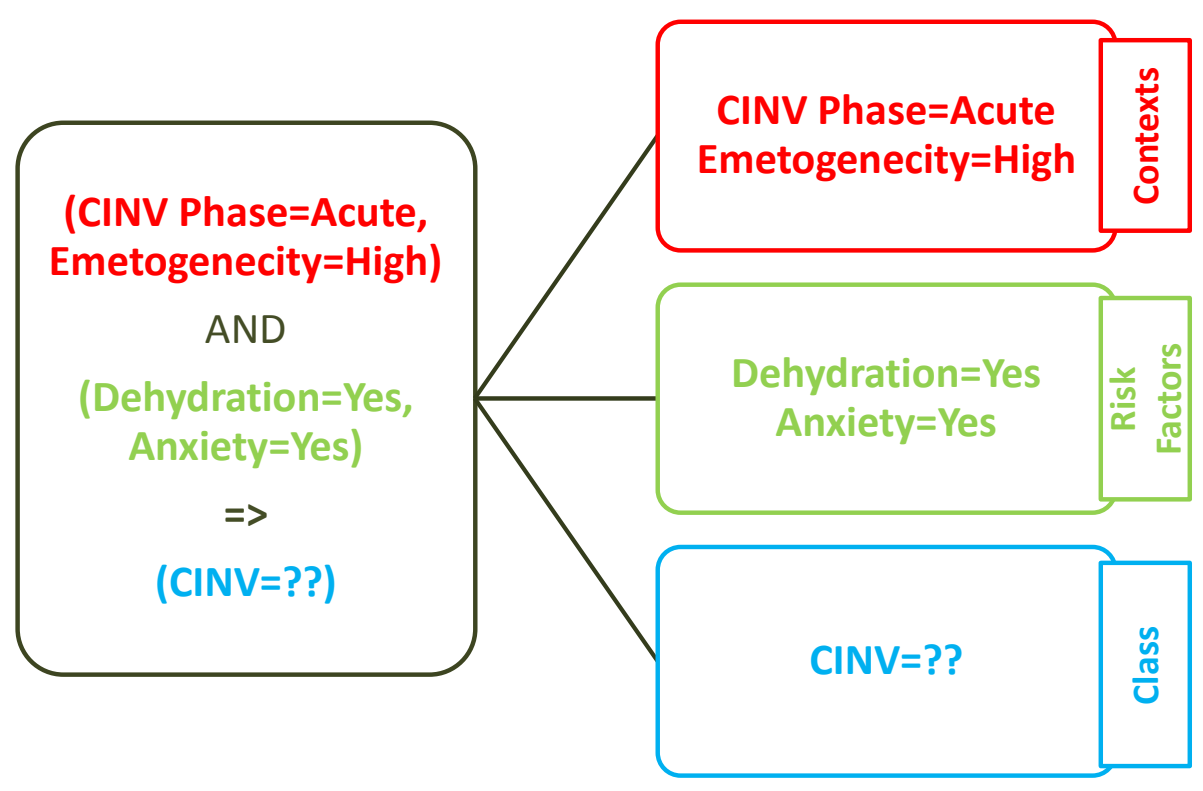

Figure 62: Prediction Query Processor

\subsubsection{Rule Extractor}

Algorithm 4 shows the rule extractor process. This is a two-step process (see Figure 63). In summary, for each rule in the rule database, if the contexts of the rule matches the contexts of the query (the first step) and the items of the rule is a subset of the risk factors in the prediction query (the second step), then the rule is marked as qualified. All qualified rules are used in the process of the dynamic prediction. Figure 64 illustrates a rule extraction example. In this example, there are five rules in the database and three rules are qualified for the prediction query. 


\section{Step 1: Match for Contexts}

\section{Step 2: Match for items}

-Hash Mapping is used for faster matching

Figure 63: Rule Extractor Steps

\section{Algorithm 4: Rule Extractor}

Input:

- $\quad$ predictionQuery, a prediction query containing contexts and riskFactors

- $\quad$ csACR, all context sensitive associationve classification rules

procedure ruleExtractor (predictionQuery, csACR)

(1) Create a ruleSet

(2) For each rule in csACR do the following

a. If contexts of the rule matches contexts of the predictionQuery and items of the rule is a subset of the riskFactors in the prediction query then add the rule to the ruleset

(3) Return ruleSet 


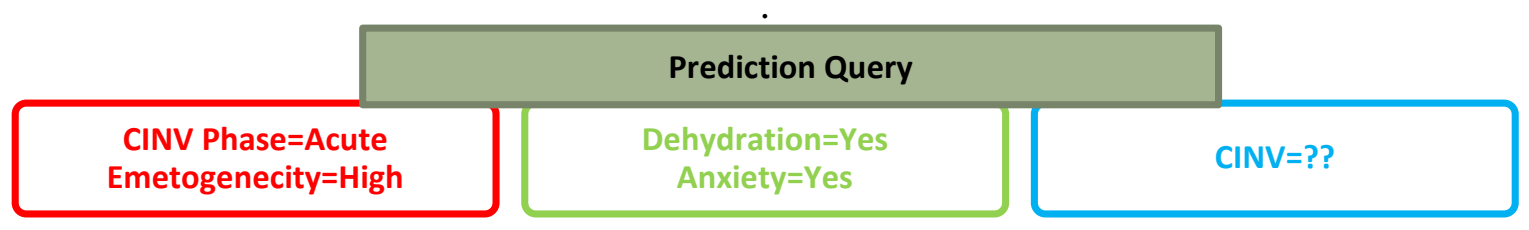

\section{Context Sensitive Associative Classification Rules}

[CINV Phase=Acute, Emetogenecity=High] [Dehydration=Yes] $\Rightarrow$ [CINV=Yes]

[CINV Phase=Acute, Emetogenecity=High] [Anxiety=Yes] $=>[\mathrm{CINV}=\mathrm{Yes}]$

[CINV Phase=Acute, Emetogenecity=High] $[$ Smoking $=\mathrm{No}]=>[\mathrm{CINV}=\mathrm{No}]$

[CINV Phase=Acute, Emetogenecity=High] [Dehydration $=Y$ Yes, Anxiety $=\mathrm{Yes}]=>[\mathrm{CINV}=\mathrm{Yes}]$

[CINV Phase=Acute, Emetogenecity=MEC] [Anxiety=Yes] $=>[\mathrm{CINV}=\mathrm{Yes}]$

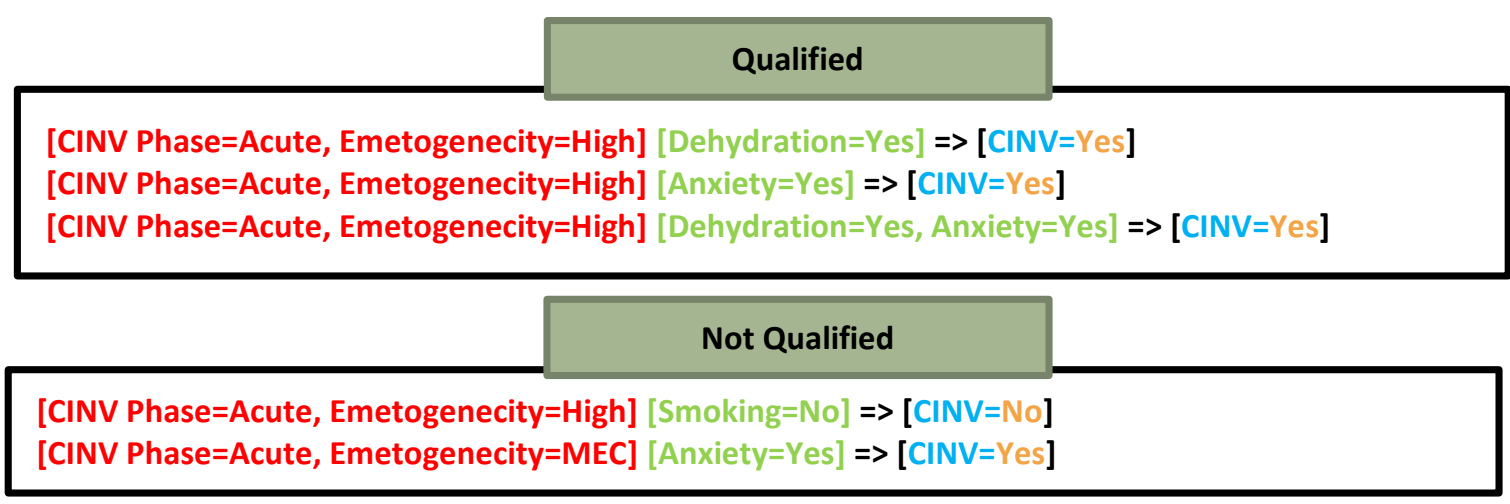

Figure 64: Rule Extractor Example

\subsubsection{Multi-Rule Dynamic Predictor}

The multi-rule predictor uses all qualified rules for making a prediction recommendation. If all qualified rules recommend the same class, then the class is returned as prediction. In the example in Figure 65, all the qualified rules refer to CINV=Yes; thus, the prediction outcome is $\mathrm{CINV}=$ Yes. Otherwise, a prediction score is calculated for each class based on the qualified rules per class. 


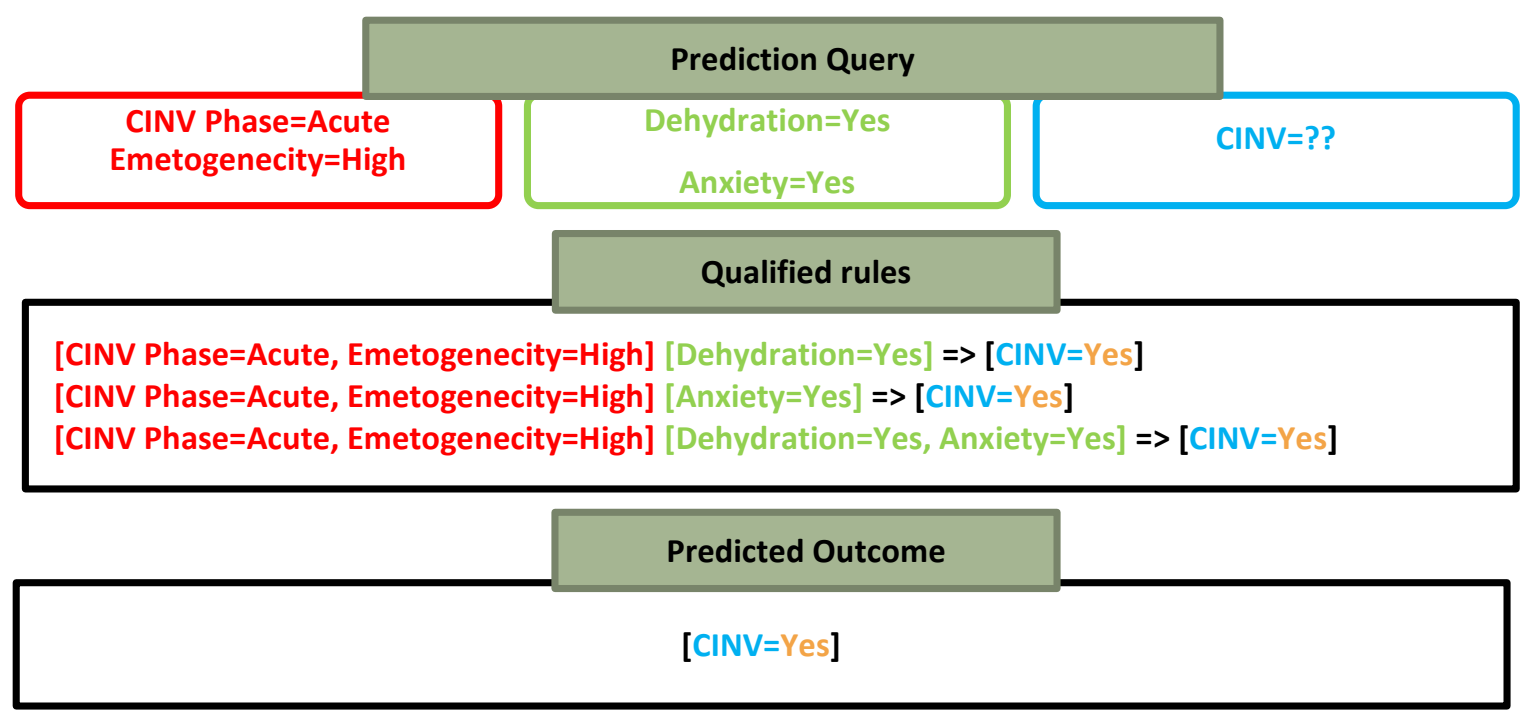

Figure 65: Multi-Rule Predictor Example

\subsection{PREDICTION SCORE}

After extracting all the qualified rules for a prediction query, there could be two scenarios: (1) all the qualified rules recommend the same class, and (2) multiple classes are recommended by the qualified rules. For the first scenario, the prediction result is straightforward as only a single class is recommend. However, for the second scenario, the system needs to know which class has the highest impact so that a prediction result can be generated. The impact of a recommended class is estimated by computing a prediction score for the class. The prediction score is estimated by taking the sum of quality measures of all qualified rules within a group (Equation 7). We considered three major association rule measures (support, confidence and lift) as well as their combinations in a weighted manner yielding additional four measures: support*confidence, support*lift, 
lift*confidence, and support*lift*confidence. For all calculation, context sensitive measures were used.

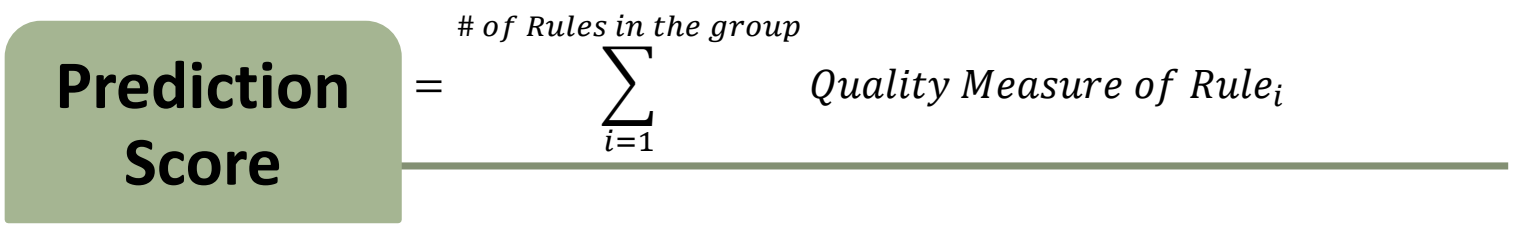

\section{Equation 7: Prediction Score}

\subsection{TRAINING STEP}

There are two steps in the training process. In the first step, context sensitive associative classification rules are discovered from the training dataset using Algorithm 2. These rules are stored in a rule database. In the second step, unnecessary rules are pruned. We considered two types of pruning: (a) pruning complementary rules, and (b) pruning weak rules.

In the rule database, many associative classification rules are conflicting or complementary. For example, for binary classification, if one rule is discovered with $80 \%$ of confidence, there is another rule with the same antecedent but different class label with $20 \%$ of confidence; such as, (Dehydration $=$ Yes AND Anxiety $=$ Yes $) \Rightarrow(\mathrm{CINV}=\mathrm{Yes})$ [Conf: 80\%] and (Dehydration=Yes AND Anxiety=Yes) $\Rightarrow(\mathrm{CINV}=$ No) [Conf:20\%]. This situation is conflicting or complementary. We used a confidence threshold (> 50\%) to prune those rule. This is done at the rule generation step without requiring any extra processing step.

As described in Section 5.1.3.3, a value of 1 for lift represents that the antecedent (LHS) is independent (LHS) of the consequent (RHS). Also, a value of less than $1 \mathrm{for}$ lift 
represents a weaker association. Thus, any rules with the value of lift less than or equal to 1 are pruned. This is also done immediately after the rule generation. Context sensitive lift can prune more rules than the lift measure within the global context. Figure 66 presents the reduction of the number of rules on a class-imbalanced dataset.

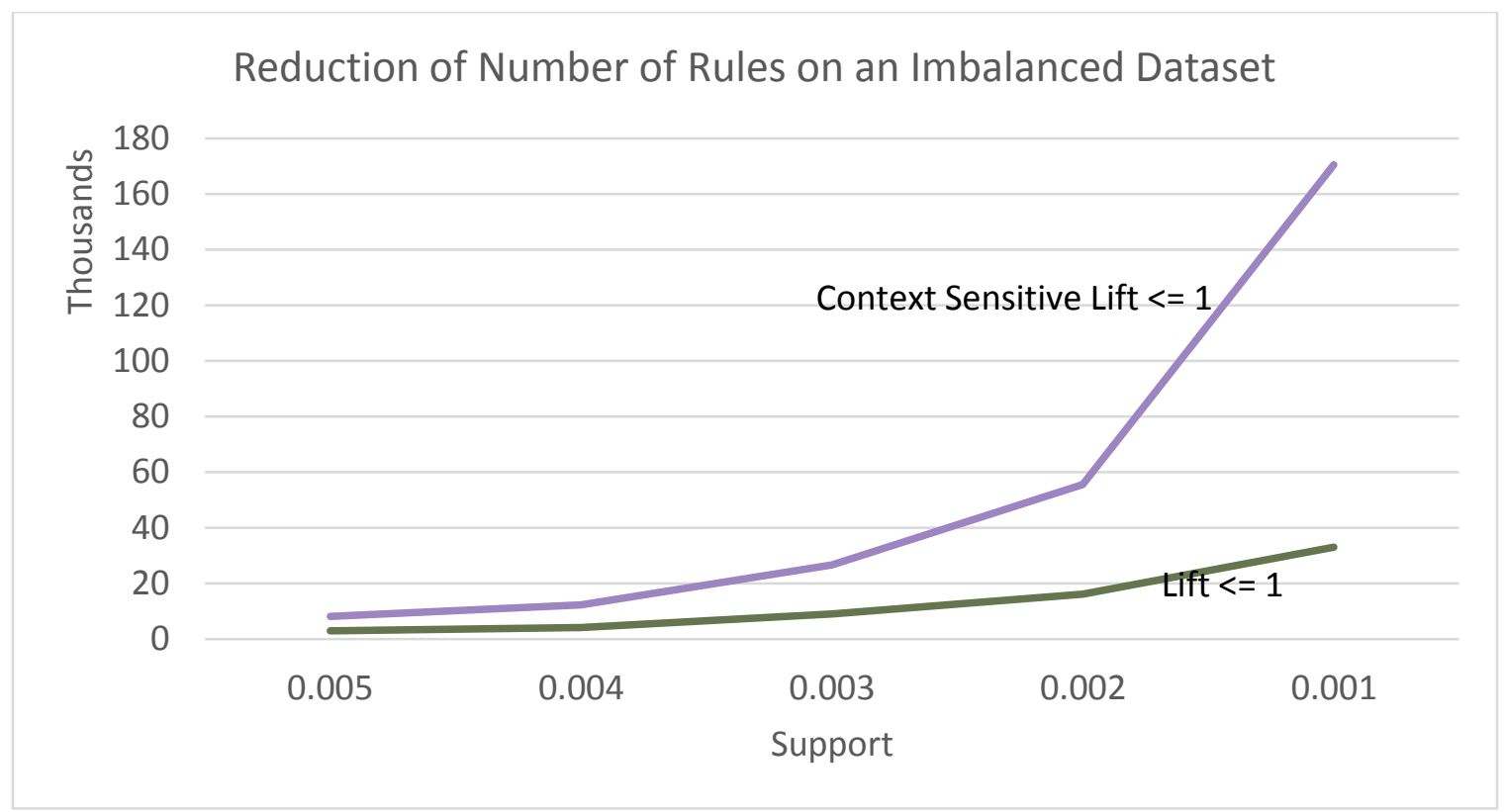

Figure 66: Reduction of Number of Rules on an Imbalanced Dataset

\subsection{DYNAMIC PREDICTION STEPS}

\subsubsection{Extracting Rules}

The rule extractor component is used for extracting the rules that are qualified for the prediction query (see section 6.2.3). Those queries are then passed to the multi-rule predictor for making a dynamic prediction recommendation. 


\subsubsection{Dynamic Prediction}

For making a dynamic recommendation based on multiple qualified rules, we experimented two different approaches: (1) all rule approach, and (2) rule ranking approach.

\subsubsection{All Rule Approach}

In this approach, all the qualified rules are used in the prediction process (Figure 67). If all the rules recommend the same class, then the class is returned as prediction. Otherwise, the rules are grouped based on class (here, there are only two groups) and then a prediction score is computed from the rules in each group. The class with the highest prediction score is recommended as the prediction outcome.

\section{Extract all qualified rules}

Group the rules based on class

Compute prediction score from the rules in each group

Class with the highest prediction score is considered as the predicted class

Figure 67: Multi-Rule Dynamic Predictor -All Rule Approach 


\subsubsection{Rule Ranking Approach}

In the rule ranking approach, top $\mathrm{n}$ rules are used in the prediction process instead of using all qualified rules (Figure 68). First, the rules are ranked based on association rule measures (discussed below). Second, top $\mathrm{n}$ rules are then used for prediction similar to the all rule approach.

In order to develop a rule ranking mechanism, we compared four association rule measures. These measures are: (1) context sensitive support, (2) context sensitive confidence, (3) context sensitive lift, and (4) rule length. Here, support refers to the prevalence of the rule in the database, confidence refers to the accuracy of the rule and lift indicates the correlation between the antecedent and the consequent of the rule. Figure 71 presents the rule ranking mechanism by comparing confidence at the first level, support at the second level, lift at the third level, and rule length at the fourth level. This rule ranking approach has been adopted in various associative classification algorithms $[118,128]$ in which only confidence, support and rule length are compared. In our approach, we used the lift measure in addition to those measures. 
Extract all qualified rules

Rank all the qualified rules, pick top $\mathrm{n}$ rules for classification

Group the rules based on class

Compute prediction score from the rules in each group

Class with the highest prediction score is considered as the predicted class

Figure 68: Multi-Rule Dynamic Predictor -Rule Ranking Approach 


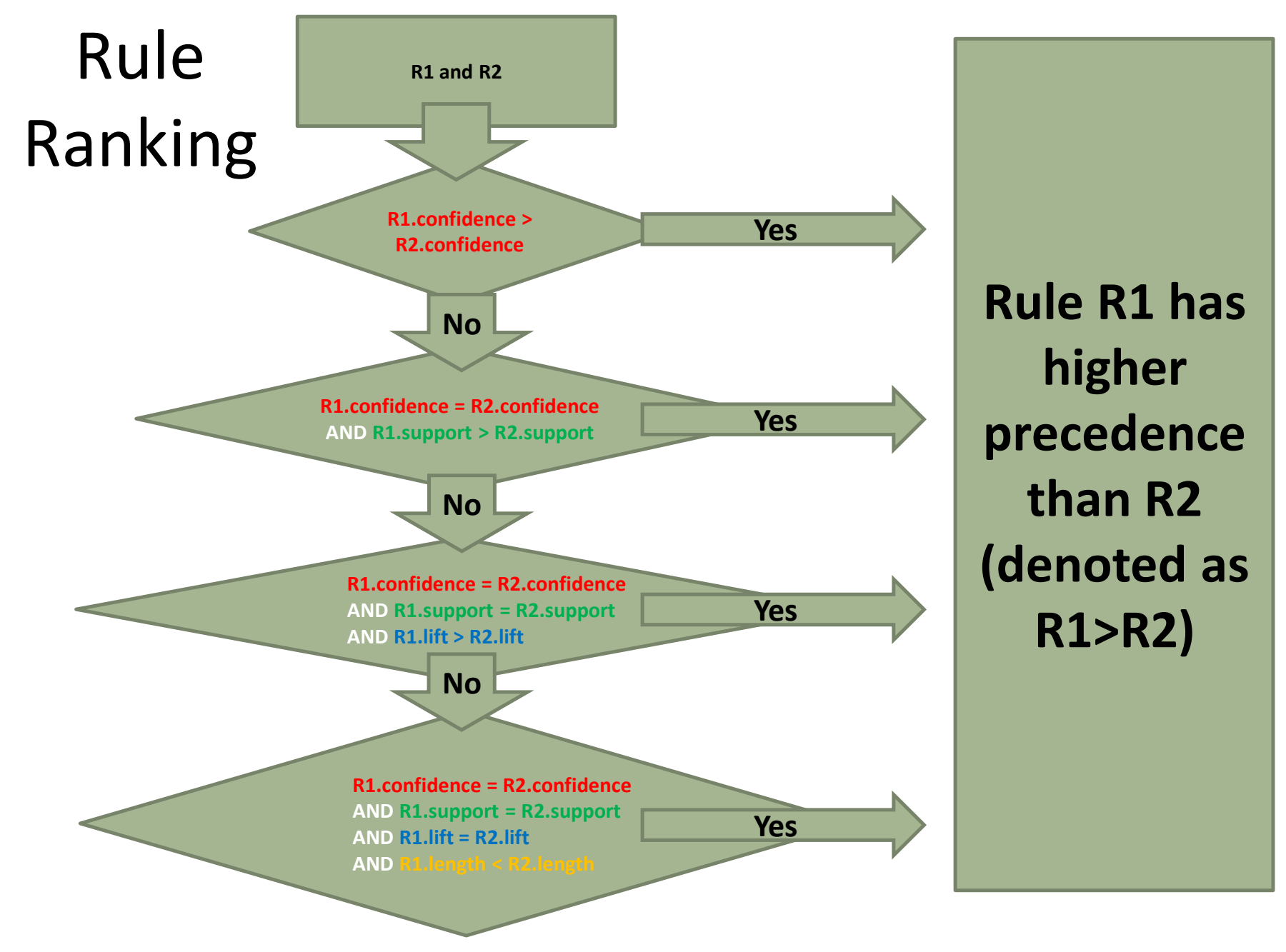

Figure 69: Rule Ranking 


\subsection{SYSTEM VALIDATION AND RESULTS}

Three performance measures were compared for benchmarking: accuracy, sensitivity, and specificity. 10-fold cross validation was used to compute the three measures.

\subsubsection{Benchmarking All Rule Approach}

We experimented the all rule approach for various support and confidence thresholds. We used five measures for computing prediction scores: (1) each rule has similar impact (i.e., 1 is added to the score for each rule), (2) confidence, (3) support * confidence, and (4) lift* confidence, and (5) support * lift * confidence. We observed that lower support and higher confidence provide higher accuracy, sensitivity, and specificity (Figure 71). The highest accuracy, sensitivity and specificity are observed at support $=0.003$, and confidence $=0.9$ when the lift*confidence measure was used. The accuracy, sensitivity, and specificity were $87.6 \%, 87.0 \%$, and $88.2 \%$ respectively. 

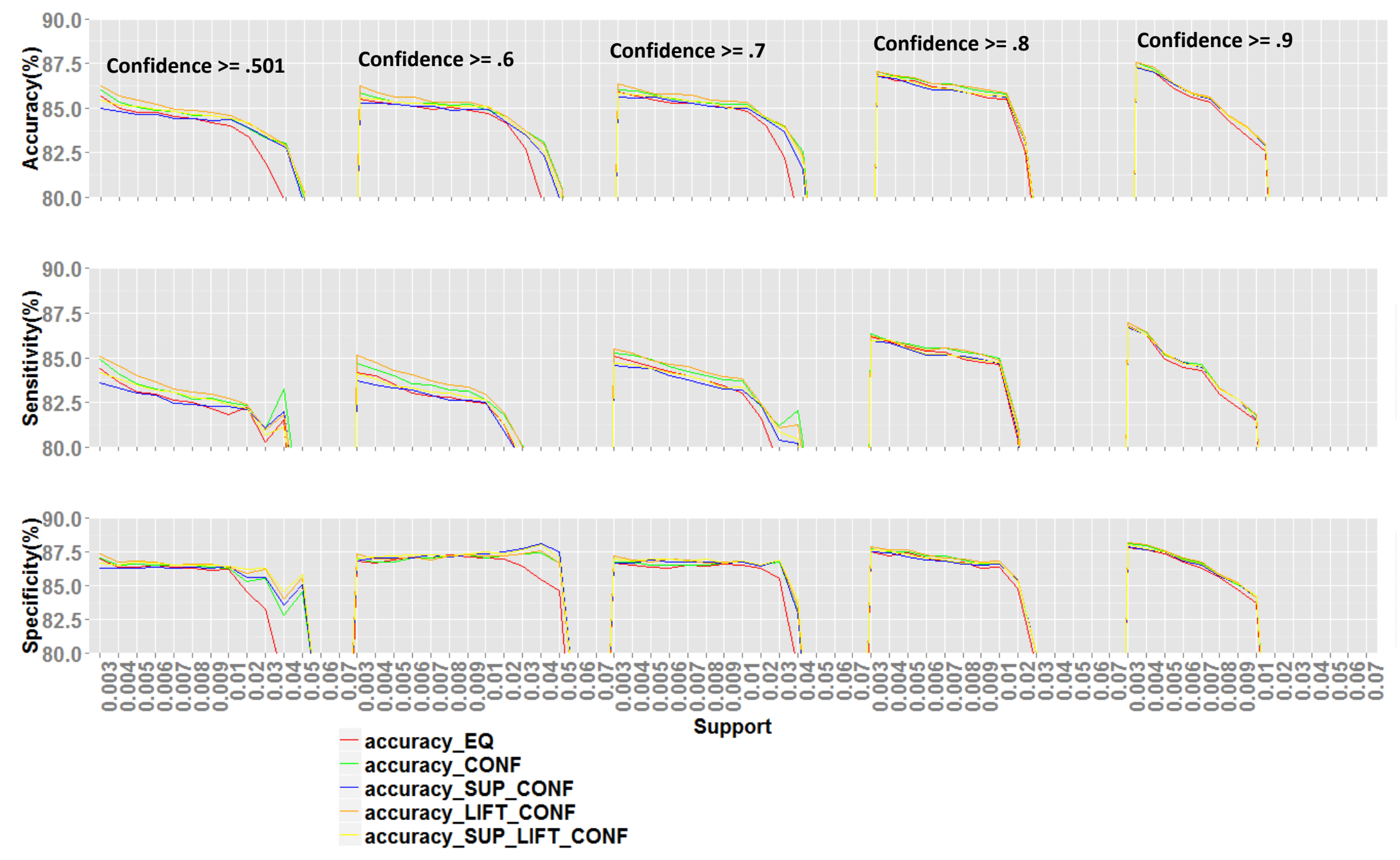

Figure 70: Benchmarking - All Rule Approach 


\subsubsection{Benchmarking Rule Ranking Approach}

We also benchmarked the rule ranking approach for top 1 to top 101 rules with 2 intervals (i.e., all the odd numbers between 1 to 101). The best result was observed when top 9 rules and the ranking mechanism of the "confidence-support-lift-rule length" (see Figure 71) were used. The accuracy, sensitivity, and specificity were $87.85 \%, 87.54 \%$, and $88.2 \%$ respectively.

Both the all-rule approach and the rule-ranking approach outperformed mostfrequently used classifiers in this field (Figure 71): (1) Neural Network, (2) Logistic regression, (3) Support Vector Machine, (4) Naïve Bayes and, and (5) Decision Tree. We used those classification algorithms in Weka [111] for this comparison. In addition, the rule ranking approach outperformed the all-rule approach. In the all-rule approach, the best performance was observed for lower support but higher confidence threshold. Determining the threshold for support and confidence is a trial-and-error approach. Instead, the rule ranking approach is preferable. 


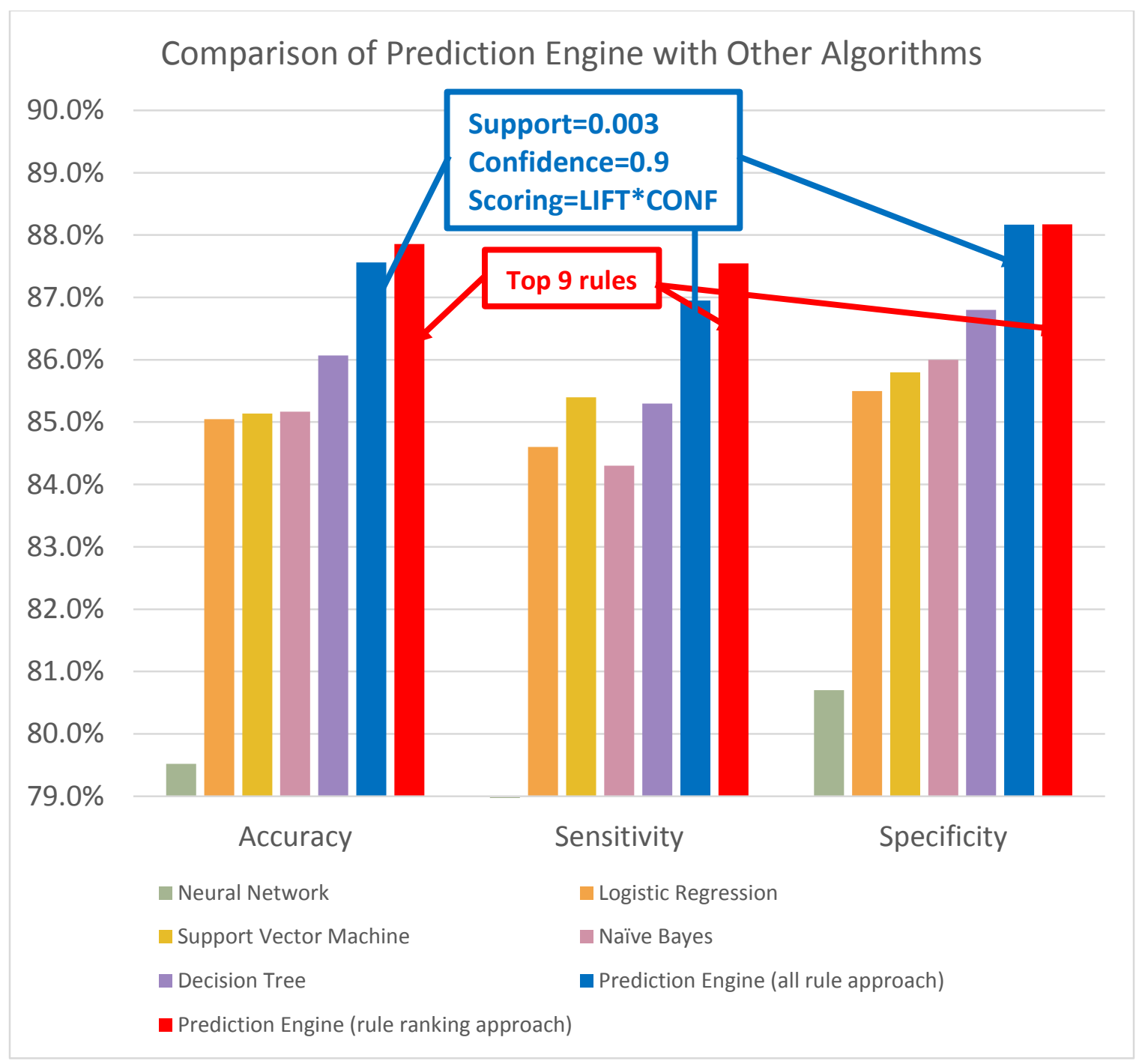

Figure 71: Comparison of Prediction Engine with Other Algorithms

\subsubsection{Prediction Using Any Number of Variables}

Traditional classification/prediction approaches require the same variables used in a classification model when an object or instance is predicted. If a variable used in a classification model is missing for some reason when an object is predicted, traditional approaches cannot predict the object without the variable (or they must build a new classification model from training data without the variable). For example, the "prior CINV" variable is not available for the first cycle of chemotherapy (Figure 72). Other 
examples could be dehydration and anxiety status that are often unreported at the point-ofcare. The prediction engine does not suffer from this issue that is common in a clinical setting as the system preserves the complete knowledge as rules from the training data and processes the prediction query in real-time. Although the top risk factor (e.g., dehydration in Figure 70) is missing, the system is able to predict a case without the top risk factor based on other variables. As a result, the system produces very high prediction performance in those situations.

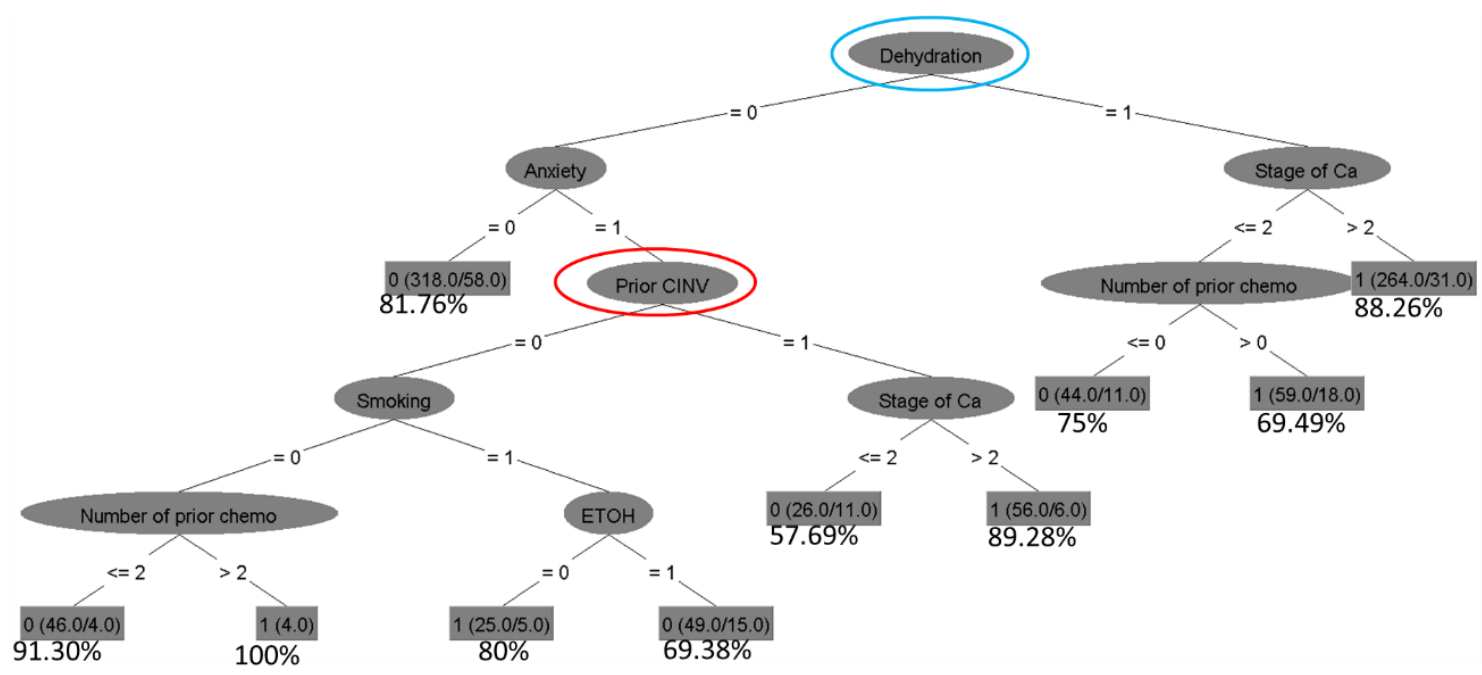

Figure 72: Loss of prediction capacity - Unavailable or Unreported Variable Situation

Filling a missing variable in a prediction query could be a solution to this issue. However, this is not feasible at the point-of-care because the predicted value of the missing variable could significantly affect the prediction. In order to compare the prediction performance of a traditional approach (i.e., the decision tree algorithm in Weka) with the prediction engine, we modified a prediction query so that the missing variable had one of the possible values of the variable for the traditional approach as a "fixed" model because without filling out the missing value it does not work. However, the original query was 
input into the prediction engine. We performed total seven comparison experiments: (1) Figure 73 shows a comparison of the prediction performance without dehydration, (2) Figure 74 is for a case without anxiety, (3) Figure 75 is without prior_cinv (4) Figure 76 is without both dehydration and anxiety, (5) Figure 77 is without dehydration and prior_cinv, (6) Figure 78 is without anxiety and prior_cinv, and (7) Figure 79 is without dehydration, anxiety and prior_cinv. In all the cases, the traditional approach showed either higher sensitivity and far lower specificity or higher specificity and far lower sensitivity compared with the prediction engine. It is impossible to know what predicted value of the missing variable would result in high sensitivity or high specificity. Thus, this "guessing" approach does not work in a clinical setting. Unlike the traditional approach, the prediction engine showed balanced performance.

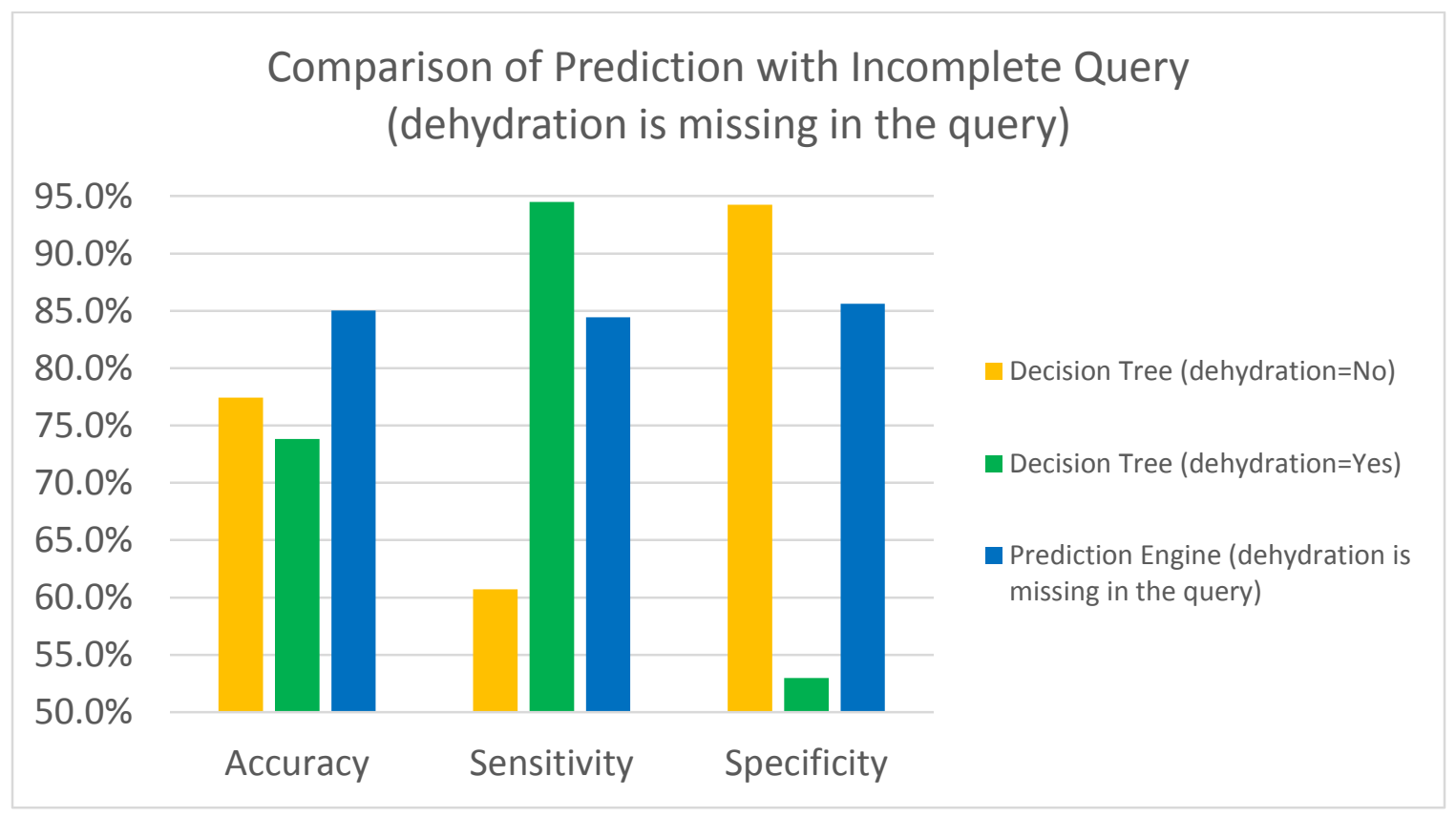

Figure 73: Comparison of Prediction with Incomplete Query (dehydration is missing in the query) 


\section{Comparison of Prediction with Incomplete Query \\ (anxiety is missing in the query)}

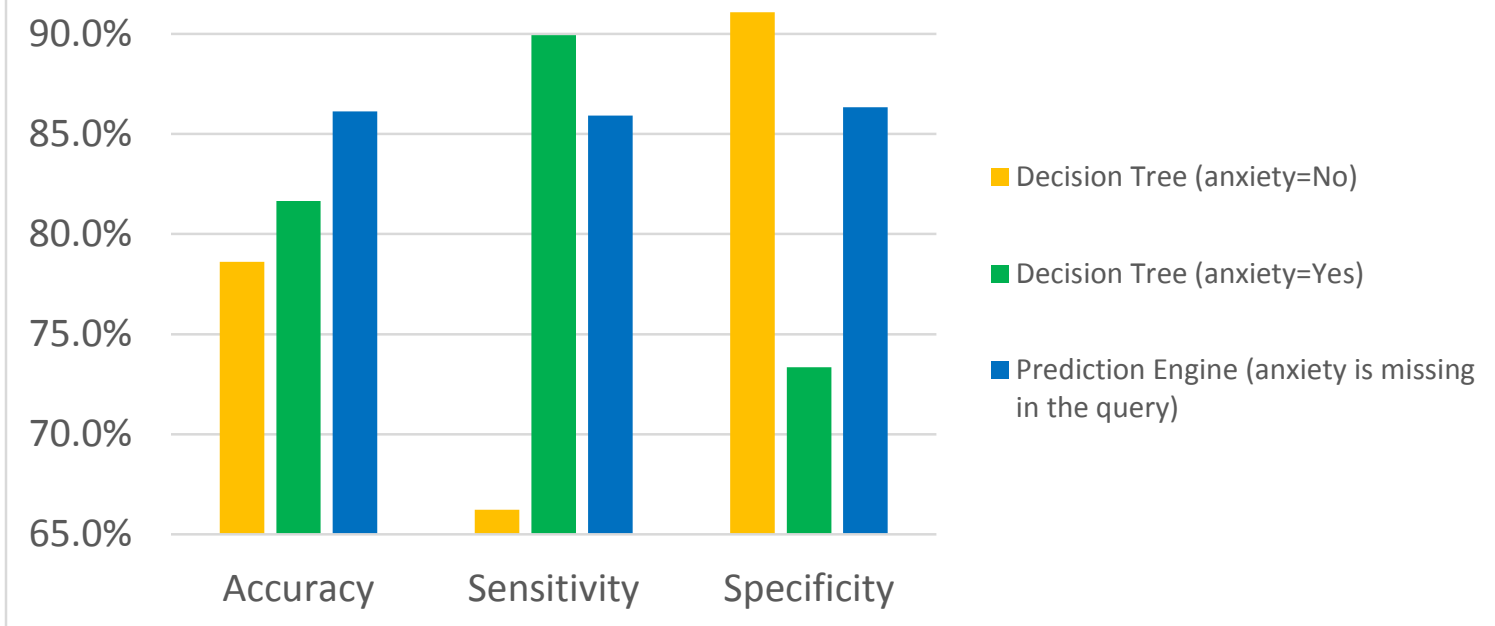

Figure 74: Comparison of Prediction with Incomplete Query (anxiety is missing in the query)

\section{Comparison of Prediction with Incomplete Query \\ (prior_cinv is missing in the query)}

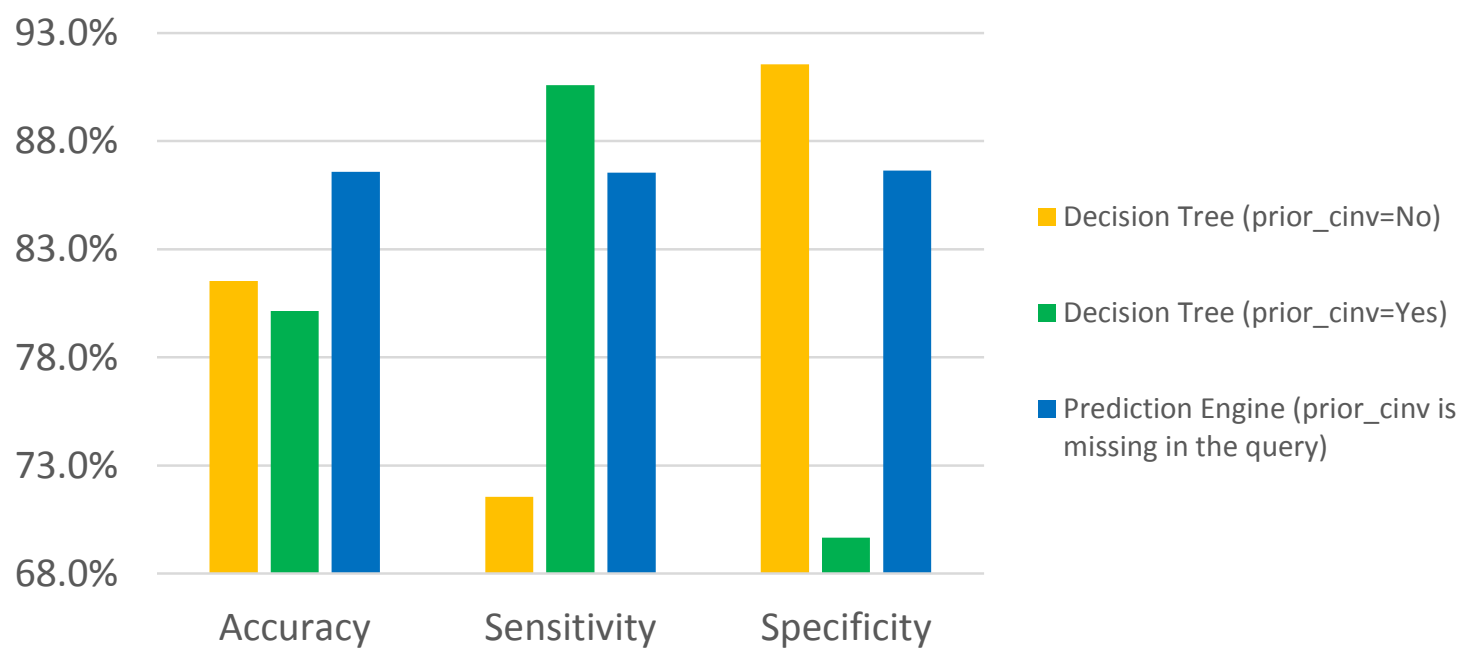

Figure 75: Comparison of Prediction with Incomplete Query (prior_cinv is missing in the query) 


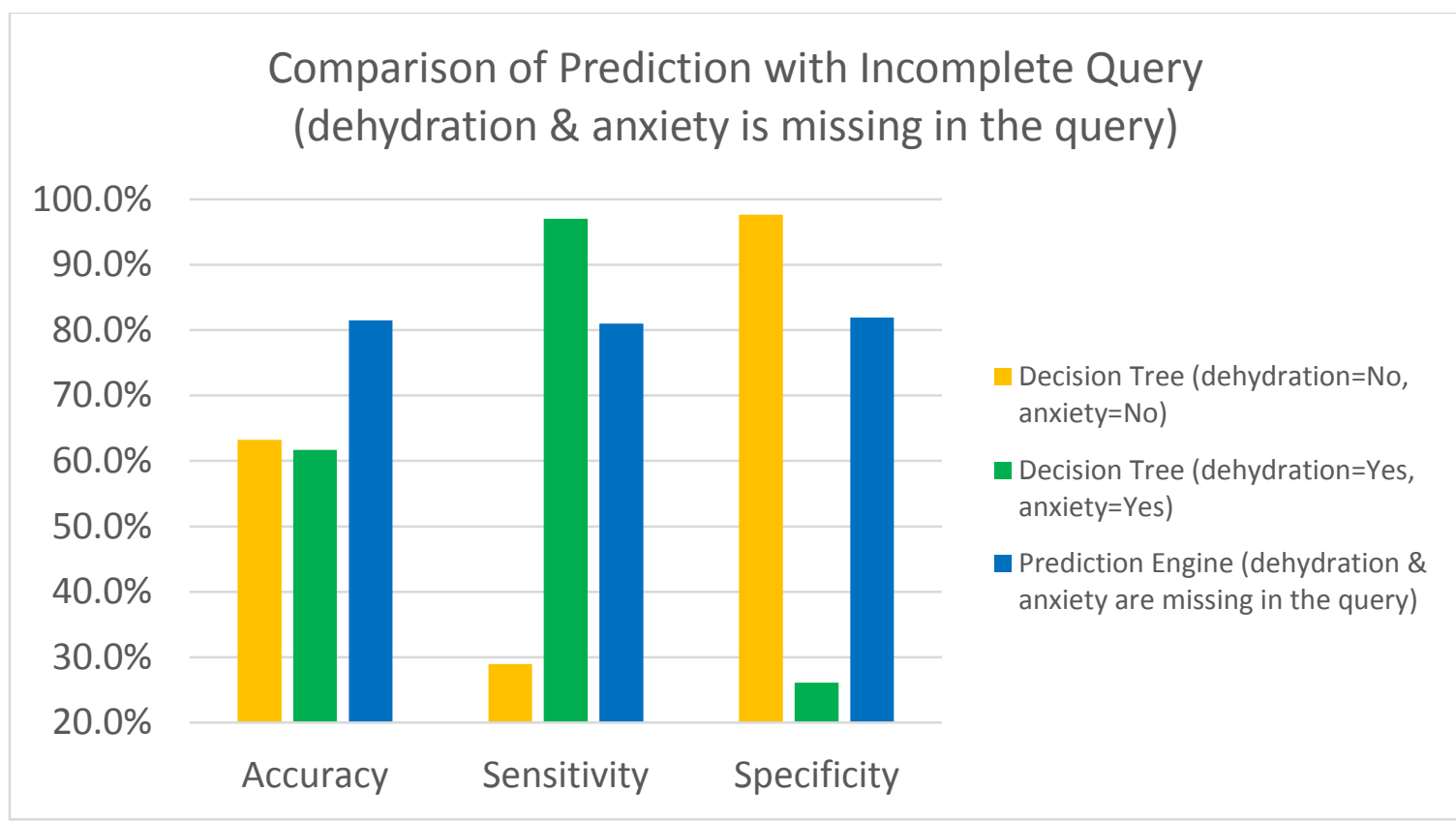

Figure 76: Comparison of Prediction with Incomplete Query (dehydration \& anxiety is missing in the query)

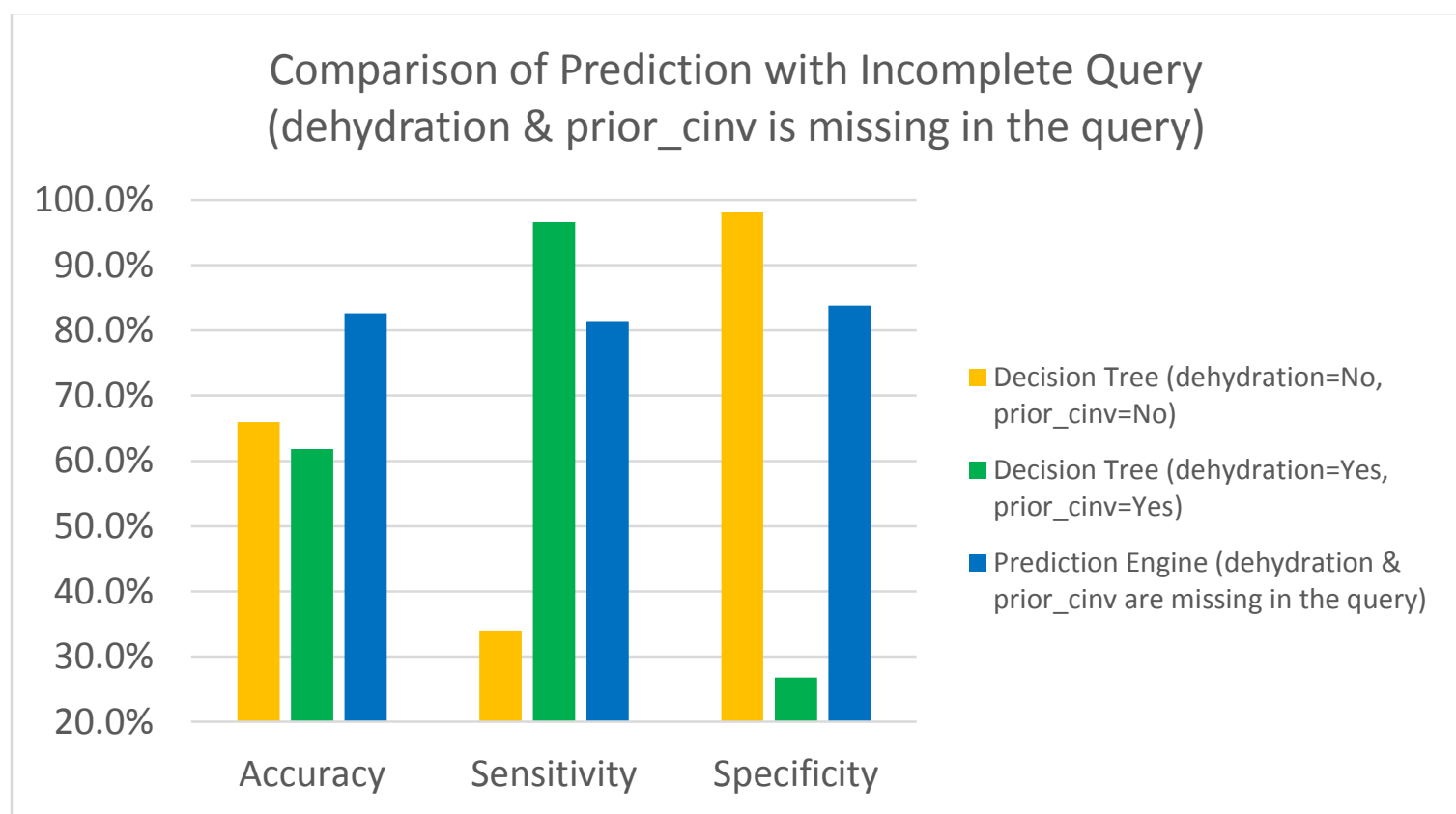

Figure 77: Comparison of Prediction with Incomplete Query (dehydration \& prior_cinv is missing in the query) 


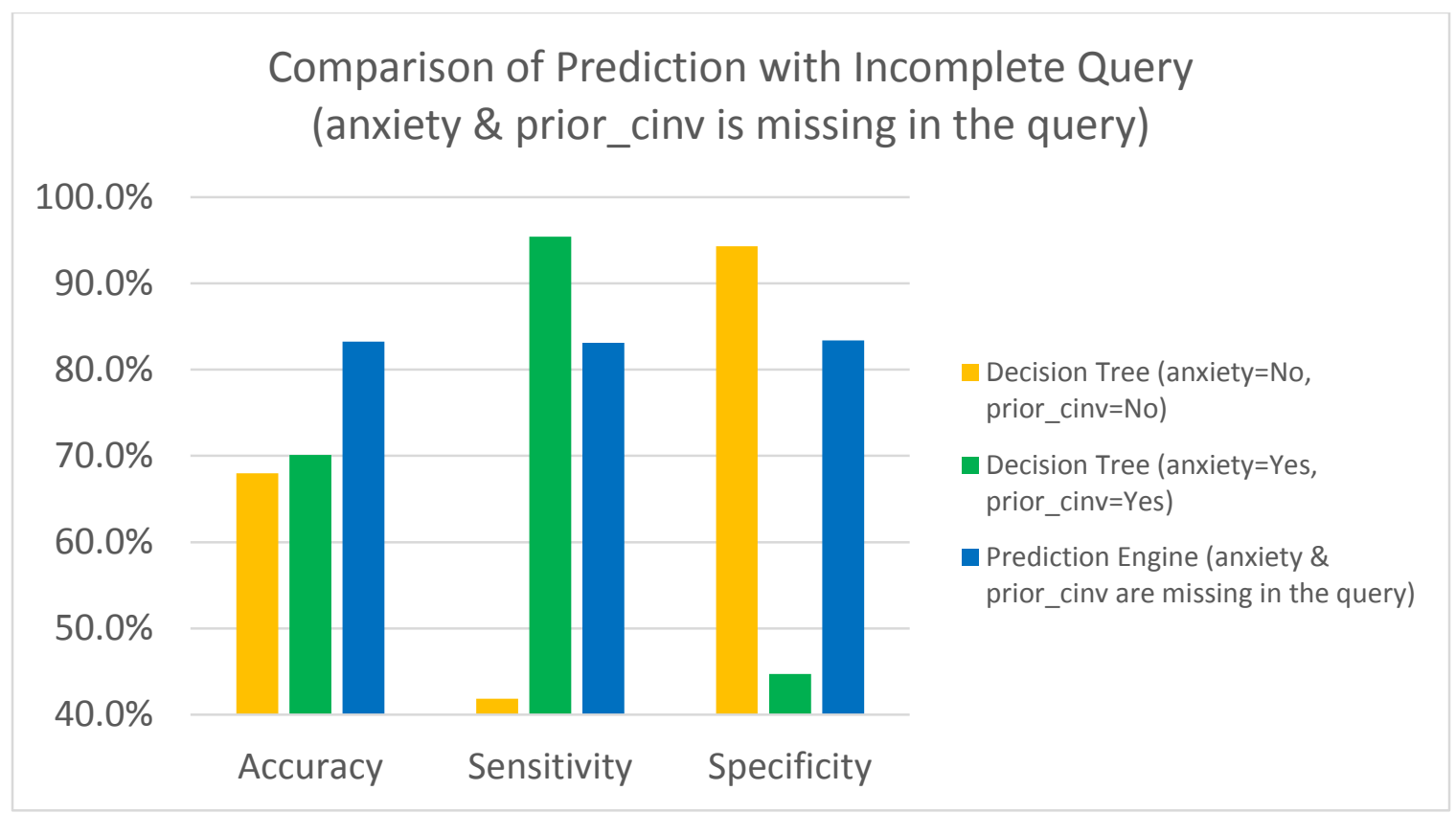

Figure 78: Comparison of Prediction with Incomplete Query (anxiety \& prior_cinv is missing in the query)

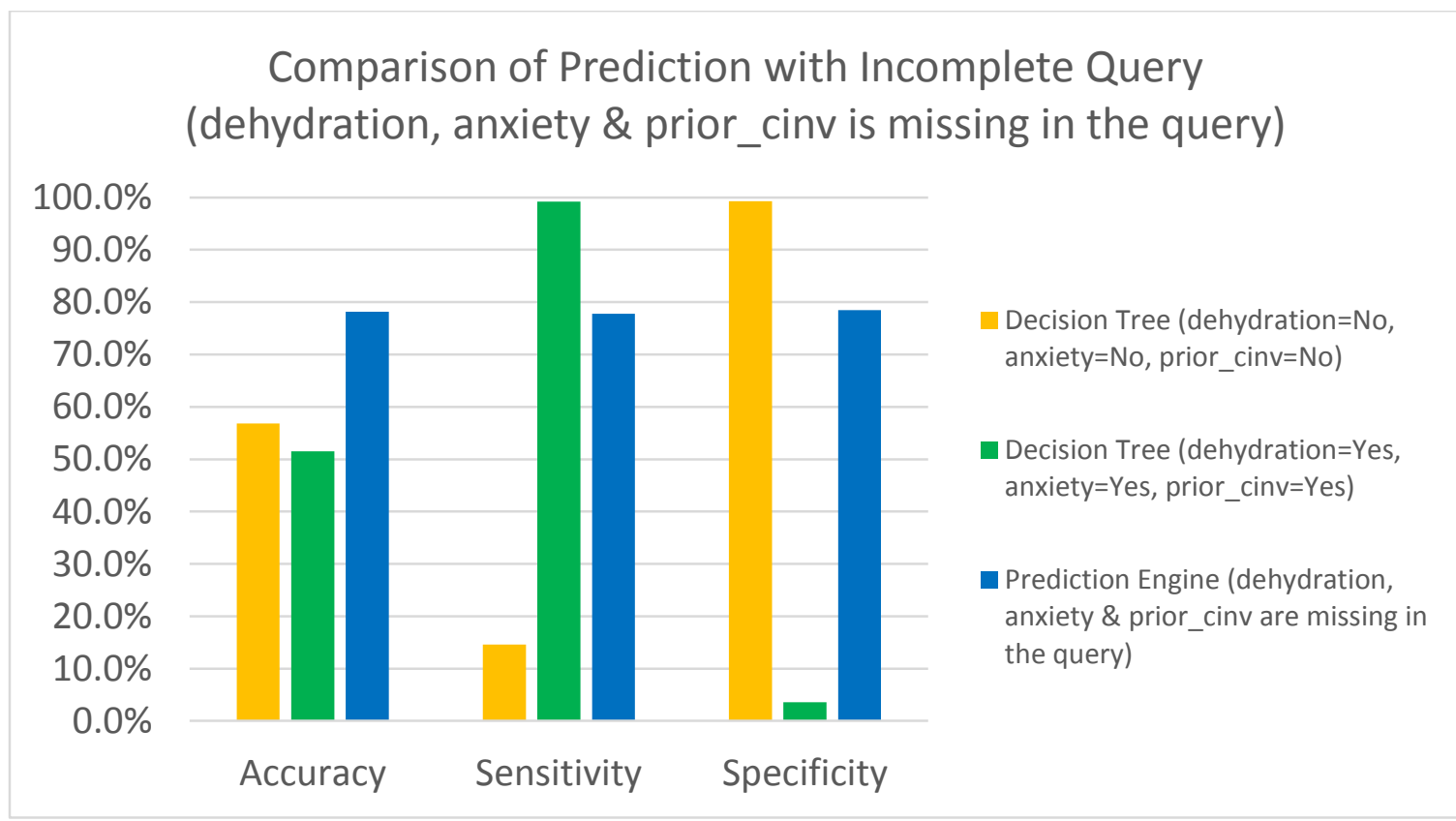

Figure 79: Comparison of Prediction with Incomplete Query (dehydration, anxiety \& prior_cinv is missing in the query) 


\section{CHAPTER 7 : DISCUSSION}

Careful examination of appropriate patient-specific risk-factors prior to selecting pre-medications for CINV is imperative in cancer care $[60,154]$. This has short term and long term effect in cancer care $[57,84,92,99]$. Better control of CINV may lead to improved tolerability of the therapy, less anxiety, higher patient's satisfaction, and avoidance of immediate discontinuation of the therapy. In the short term, better CINV control would allow for avoidance of hospitalization, less side effect of the regimen, better food and fluid intake, maintenance of baseline weight, maintenance of performance status, adherence to the interval and duration of the complete therapy, and significantly less physiological and psycho-social stress both to cancer patients and caregivers. Moreover, all these characteristics may translate to both improved progression-free survival (PFS) and overall survival (OS) [57,84,91,92,99]. There are several medical and economical long-term effects. In the long term, it may contribute to better recovery for the patients, early rehabilitation in personal, social and professional life, and a positive consideration for further chemotherapy in the future for recurrent or secondary malignancies leading to better treatment outcome. Its economic effects are enormous as they may reduce both the direct and indirect health care costs as well as reduce lost workdays [84,91,92,99].

To date, the comprehensive relationships of various patient-related factors for causing CINV were unknown. This project discovered important relationships through data mining that helps physicians for practicing personalized and precision medicine for the 
management of CINV leading to improved clinical outcomes. This project could significantly improve overall cancer care and reduce overall cancer treatment cost. We have developed a highly accurate prediction system that is dynamic and updateable in realtime. Six datasets (three chemotherapy groups and two phases of CINV in each chemotherapy group) were analyzed individually so that the prediction models were built based on the evidence from the corresponding dataset. A novel context-sensitive prediction system (named as "prediction engine") was introduced. The system does not suffer from the issue of losing prediction capacity in a situation when a set of important variables is unavailable or unknown at the point-of-care. The system has higher prediction power since multiple important rules are used for a single prediction. A new prediction scoring algorithm was designed to compute a prediction score from multiple rules. The prediction engine keeps all unimportant (rare) rules. The support value may increase for the rare rules with the addition of more data to the system in real-time. The dynamic nature of the system may benefit the unimportant or rare rules to become important as more data is added in real-time. The approach proposed in this project can be adapted for any other clinical predictions.

\subsection{CINV PREDICTION IN THE LITERATURE}

We also performed a systematic literature review in order to find related studies for CINV prediction. We followed the same search strategy as the systematic review described in Chapter 2. We found a total of 5 articles discussing three different risk prediction models of CINV. 
The first model [86] was built for the acute phase of CINV using a dataset containing 864 cycles of chemotherapy received from 200 patients and the model validation was done using a test dataset containing 695 cycles of chemotherapy received from 146 patients. This was a single center prospective study performed in Canada. The patients received either high or moderate emetogenic chemotherapy. The prescriptions for antiemetics medications were based on the ASCO guidelines. The authors of the study performed three validation studies: the first one is as part of the original study [86] and the other two prospective studies [96,101] for validating the models independently from the training data (see Table 17).

Table 17: Performance of Risk Scoring Algorithm for Acute CINV

\begin{tabular}{|c|c|c|c|c|}
\hline Study (Year) & Optimal Risk Score Cutoff & Accuracy & Sensitivity & Specificity \\
\hline Dranitsaris et al. (2009) [86] & $\geq 7$ to $<9$ & $67.4 \%$ & $78.6 \%$ & $64.8 \%$ \\
\hline \multirow{2}{*}{ Bouganim et al. (2012) [101] } & $\geq 7$ & $55.8 \%$ & $71.9 \%$ & $52.3 \%$ \\
\cline { 2 - 5 } & $\geq 9$ & $74.6 \%$ & $53.1 \%$ & $79.2 \%$ \\
\hline \multirow{2}{*}{ Dranitsaris et al. (2013) [96] } & $\geq 7$ & $60.1 \%$ & $68.5 \%$ & $58.8 \%$ \\
\cline { 2 - 5 } & $\geq 9$ & $80.3 \%$ & $86.7 \%$ & $31.3 \%$ \\
\hline
\end{tabular}

The second model [87] was built for the delayed phase of CINV using the same dataset as Dranitsaris et al. (2009) [86]. The data analysis method is same as the original study (dealing with the acute phase) while the risk scoring algorithm for the delayed phase is different than the one in the original study. The details of the algorithm are presented in Petrella et al. (2009) [87]. They performed three validation studies: the first one in the original study [87] and the other two prospective studies [96,101] for validating the models independently from the training data (see Table 18). 
Table 18: Performance of Risk Scoring Algorithm for Delayed CINV

\begin{tabular}{|c|c|c|c|c|}
\hline Author (Year) & Optimal Risk Score Cutoff & Accuracy & Sensitivity & Specificity \\
\hline Petrella et al. (2009) [87] & $>16$ to $\leq 20$ & $67.9 \%$ & $72 \%$ & $64.4 \%$ \\
\hline \multirow{2}{*}{ Bouganim et al. (2012) [101] } & $>16$ & $54.7 \%$ & $75.8 \%$ & $50.0 \%$ \\
\cline { 2 - 5 } & $>20$ & $73.5 \%$ & $57.6 \%$ & $77.0 \%$ \\
\hline \multirow{2}{*}{ Dranitsaris et al. (2013) [96] } & $>16$ & $58.6 \%$ & $77.9 \%$ & $53.3 \%$ \\
\cline { 2 - 5 } & $>20$ & $67.8 \%$ & $66.3 \%$ & $68.2 \%$ \\
\hline
\end{tabular}

The third model was developed by Molassiotis et al. (2013) [70] using a training dataset containing 791 cycles of chemotherapy received from 286 patients. The model validation was performed using a test dataset from 50 chemotherapy patients (the number of cycles in the test dataset is not disclosed in the article). This was a multicenter prospective study performed in the United Kingdom. The patients received high, moderate or low emetogenic chemotherapy. The prescriptions for antiemetics medications were based on the MASCC guidelines. This study developed three different logistic regression models: one for the acute phase of CINV, another for the delayed phase of CINV, and the other for overall CINV (both the acute and delayed phases). The details of the models are presented in Molassiotis et al. (2013) [70]. The results of the model validation are presented in Table 19.

Table 19: Performance of three CINV logistic regression models by Molassiotis et al. (2013) [70]

\begin{tabular}{|c|c|c|c|}
\hline CINV Phase & Accuracy & Sensitivity & Specificity \\
\hline Acute & Not reported & $76.1 \%$ & $48.9 \%$ \\
\hline Delayed & Not reported & $80.4 \%$ & $47.2 \%$ \\
\hline Overall (Acute and Delayed) & Not reported & $79.0 \%$ & $50.0 \%$ \\
\hline
\end{tabular}


Figure 80 compares the prediction performance of the prediction engine with the prediction performance published in the literature (shown in Table 17, Table 18, and Table 19). Only one study attempted to produce a combined model for both acute and delayed phases of CINV. Rest of the studies developed and reported their models individually. The prediction performance of the proposed prediction engine was reported for both the acute and delayed phases together (see context-sensitive prediction in Chapter 6). The prediction engine produced a high performance in comparison to the prediction results from the literature. Only one study [96] reported $86.7 \%$ of a sensitivity, which is comparable to the proposed approach. However, the high sensitivity was archived at the cost of the very low specificity (31.3\%). Overall, we believe that the prediction engine outperforms all the models reported in the literature for CINV prediction. 


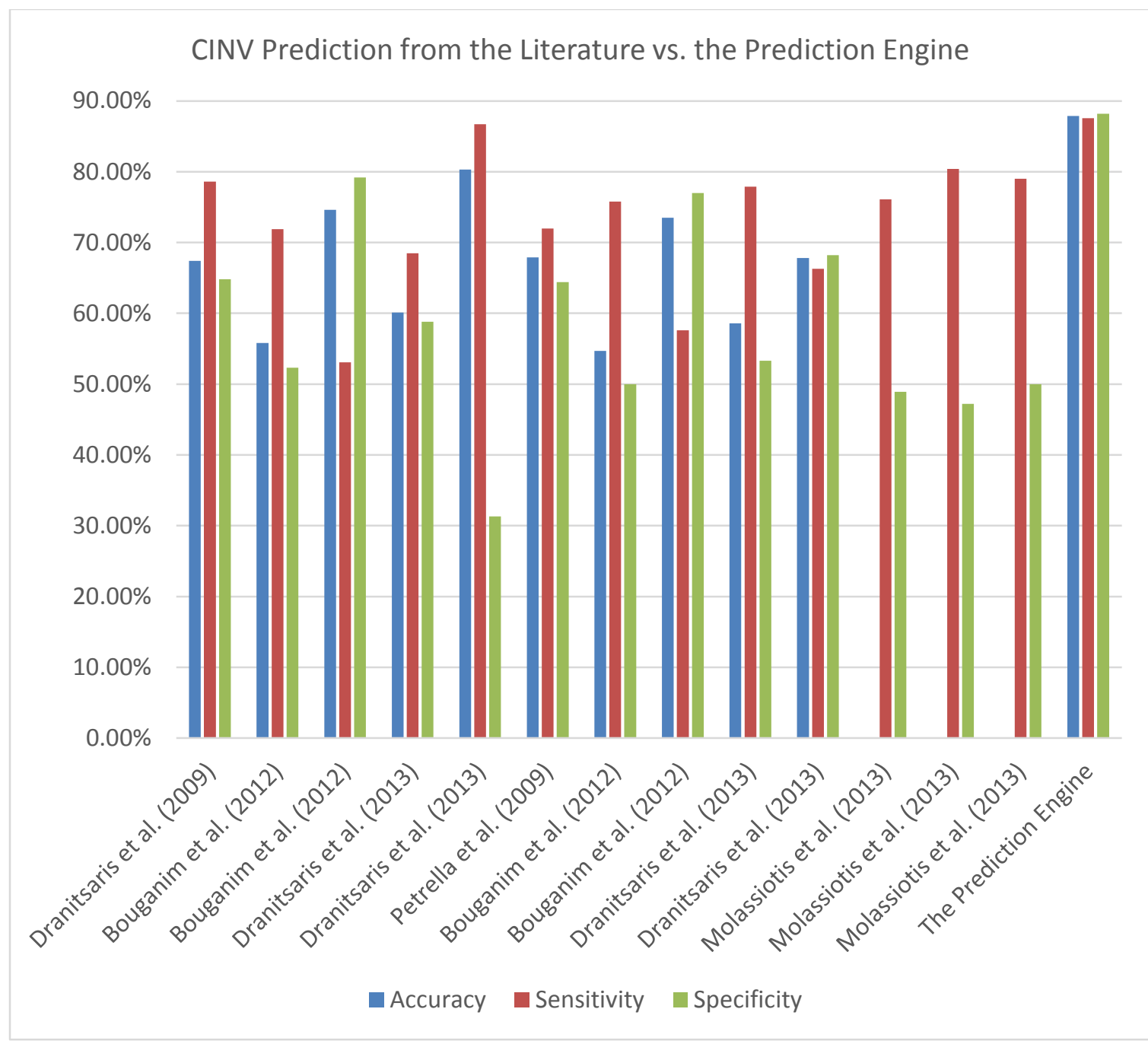

Figure 80: CINV Prediction from the Literature vs. the Prediction Engine

\subsection{LIMITATIONS}

The project has a few limitations. First, the data was collected by retrospective record review. A prospective validation is needed to confirm the usefulness of the model in real clinical setting. Second, studies showed that female patients with pregnancy-related nausea and vomiting have a higher risk of CINV. However, this information was missing in our dataset. With this information, the prediction results could be considerably enhanced. Third, the data has a lower representation of Asians and Hispanics. A multi-center and/or 
multi-national study including various populations is needed to overcome this shortcoming.

\subsection{CONCLUSION}

The prediction engine presented in this dissertation has the capacity to build a highly precise prediction system while preserving the context-sensitiveness in one single system. The prediction performance of the system outperformed many popular prediction methods and all the results of CINV risk prediction published in the literature. We used the patient-specific risk factors for making personalized treatment recommendations for CINV. This project solved a real clinical problem that will shorten the gap between clinical

practices and evidence-based guidelines for CINV management leading to the practice of personalized and precise treatment recommendation, better life quality of patient and reduced healthcare cost. 


\section{BIBLIOGRAPHY}

[1] Edwards BK, Ward E, Kohler BA, Eheman C, Zauber AG, Anderson RN, Jemal A, Schymura MJ, Lansdorp-Vogelaar I, Seeff LC, van Ballegooijen M, Goede SL, Ries LAG. Annual report to the nation on the status of cancer, 1975-2006, featuring colorectal cancer trends and impact of interventions (risk factors, screening, and treatment) to reduce future rates. Cancer. 2010;116(3):544-73.

[2] Berry DA, Cronin KA, Plevritis SK, Fryback DG, Clarke L, Zelen M, Mandelblatt JS, Yakovlev AY, Habbema JDF, Feuer EJ, Cancer Intervention and Surveillance Modeling Network (CISNET) Collaborators. Effect of screening and adjuvant therapy on mortality from breast cancer. N. Engl. J. Med. 2005;353(17):1784-92.

[3] Etzioni R, Tsodikov A, Mariotto A, Szabo A, Falcon S, Wegelin J, DiTommaso D, Karnofski K, Gulati R, Penson DF, Feuer E. Quantifying the role of PSA screening in the US prostate cancer mortality decline. Cancer Causes Control. 2008;19(2):175-81.

[4] Raimondi S, Lowenfels AB, Morselli-Labate AM, Maisonneuve P, Pezzilli R. Pancreatic cancer in chronic pancreatitis; aetiology, incidence, and early detection. Best Pract. Res. Clin. Gastroenterol. 2010;24(3):349-58.

[5] Cancer Facts \& Figures 2013. Atlanta, GA: American Cancer Society; 2013.

[6] DeSantis CE, Lin CC, Mariotto AB, Siegel RL, Stein KD, Kramer JL, Alteri R, Robbins AS, Jemal A. Cancer treatment and survivorship statistics, 2014. CA. Cancer J. Clin. 2014;64(4):252-71.

[7] Siegel R, Naishadham D, Jemal A. Cancer statistics, 2013. CA. Cancer J. Clin. 2013;63(1):11-30.

[8] Siegel R, Naishadham D, Jemal A. Cancer statistics, 2012. CA. Cancer J. Clin. 2012;62(1):10-29.

[9] Howlader N, Noone A, Krapcho M, Garshell J, Neyman N, Altekruse S, Kosary C, Yu M, Ruhl J, Tatalovich Z, Cho H, Mariotto A, Lewis D, Chen H, Feuer E, Cronin K, editors. SEER Cancer Statistics Review, 1975-2010. Bethesda, MD: National Cancer Institute; 2013. 
[10] Siegel RL, Miller KD, Jemal A. Cancer statistics, 2015. CA. Cancer J. Clin. 2015;65(1):5-29.

[11] Siegel R, Ma J, Zou Z, Jemal A. Cancer statistics, 2014. CA. Cancer J. Clin. 2014;64(1):9-29.

[12] Murphy SL, Xu J, Kochanek KD. Deaths: Final data for 2010. National vital statistics reports. Hyattsville, MD: National Center for Health Statistics; 2013.

[13] Schiller JS, Lucas JW, Peregoy JA. Summary health statistics for U.S. adults: National Health Interview Survey, 2011. Vital and Health Statistics. Hyattsville, MD: National Center for Health Statistics; 2012.

[14] Hanahan D, Weinberg RA. Hallmarks of cancer: the next generation. Cell. 2011;144(5):646-74.

[15] Hanahan D, Weinberg RA. The hallmarks of cancer. Cell. 2000;100(1):57-70.

[16] Eckhouse S, Sullivan R. The state of academic cancer surgery in the UK. Mol. Oncol. 2008;2(3):206-12.

[17] Hawkins R, Grunberg S. Chemotherapy-induced nausea and vomiting: challenges and opportunities for improved patient outcomes. Clin. J. Oncol. Nurs. 2009;13(1):54-64.

[18] Feyer PC, Maranzano E, Molassiotis A, Roila F, Clark-Snow RA, Jordan K. Radiotherapy-induced nausea and vomiting (RINV): MASCC/ESMO guideline for antiemetics in radiotherapy: update 2009. Support. Care Cancer. 2011;19 Suppl $1:$ S5-14.

[19] DeVita VT, Chu E. A history of cancer chemotherapy. Cancer Res. 2008;68(21):8643-53.

[20] Heidary N, Naik H, Burgin S. Chemotherapeutic agents and the skin: An update. J. Am. Acad. Dermatol. 2008;58(4):545-70.

[21] Balagula Y, Lacouture ME, Cotliar JA. Dermatologic toxicities of targeted 
anticancer therapies. J. Support. Oncol. 2010;8(4):149-61.

[22] Hu C-Y, Chan W, Delclos GP, Du XL. Adjuvant chemotherapy and risk of gastrointestinal, hematologic, and cardiac toxicities in elderly patients with stage III colon cancer. Am. J. Clin. Oncol. 2012;35(3):228-36.

[23] Paus R, Haslam IS, Sharov AA, Botchkarev VA. Pathobiology of chemotherapyinduced hair loss. Lancet Oncol. 2013;14(2):e50-9.

[24] Haslam IS, Pitre A, Schuetz JD, Paus R. Protection against chemotherapy-induced alopecia: targeting ATP-binding cassette transporters in the hair follicle? Trends Pharmacol. Sci. 2013.

[25] Davila M, Bresalier RS. Gastrointestinal complications of oncologic therapy. Nat. Clin. Pract. Gastroenterol. Hepatol. 2008;5(12):682-96.

[26] Stein A, Voigt W, Jordan K. Chemotherapy-induced diarrhea: pathophysiology, frequency and guideline-based management. Ther. Adv. Med. Oncol. 2010;2(1):51-63.

[27] Sun CC, Bodurka DC, Weaver CB, Rasu R, Wolf JK, Bevers MW, Smith JA, Wharton JT, Rubenstein EB. Rankings and symptom assessments of side effects from chemotherapy: insights from experienced patients with ovarian cancer. Support. Care Cancer. 2005;13(4):219-27.

[28] Feyer P, Jordan K. Update and new trends in antiemetic therapy: the continuing need for novel therapies. Ann. Oncol. 2011;22(1):30-8.

[29] Coates A, Abraham S, Kaye SB, Sowerbutts T, Frewin C, Fox RM, Tattersall $\mathrm{MH}$. On the receiving end--patient perception of the side-effects of cancer chemotherapy. Eur. J. Cancer Clin. Oncol. 1983;19(2):203-8.

[30] Cooper S, Georgiou V. The impact of cytotoxic chemotherapy--perspectives from patients, specialists and nurses. Eur. J. Cancer. 1992;28A Suppl:S36-8.

[31] Durant JR. The problem of nausea and vomiting in modern cancer chemotherapy. CA. Cancer J. Clin. 1984;34(1):2-6. 
[32] Morrow GR. Chemotherapy-related nausea and vomiting: etiology and management. CA. Cancer J. Clin. 1989;39(2):89-104.

[33] National Cancer Institute. Common Terminology Criteria for Adverse Events (CTCAE) Version 4.03. Bethesda, MD: National Institute of Health (NIH Publication No. 09-5410); 2010.

[34] Hesketh PJ, Van Belle S, Aapro M, Tattersall FD, Naylor RJ, Hargreaves R, Carides AD, Evans JK, Horgan KJ. Differential involvement of neurotransmitters through the time course of cisplatin-induced emesis as revealed by therapy with specific receptor antagonists. Eur. J. Cancer. 2003;39(8):1074-80.

[35] Thompson N. Optimizing treatment outcomes in patients at risk for chemotherapy-induced nausea and vomiting. Clin. J. Oncol. Nurs. 2012;16(3):309-13.

[36] Basch E, Prestrud AA, Hesketh PJ, Kris MG, Feyer PC, Somerfield MR, Chesney M, Clark-Snow RA, Flaherty AM, Freundlich B, Morrow G, Rao K V, Schwartz RN, Lyman GH. Antiemetics: American Society of Clinical Oncology clinical practice guideline update. J. Clin. Oncol. 2011;29(31):4189-98.

[37] Basch E, Hesketh PJ, Kris MG, Prestrud AA, Temin S, Lyman GH. Antiemetics: american society of clinical oncology clinical practice guideline update. J. Oncol. Pract. 2011;7(6):395-8.

[38] Ettinger DS, Armstrong DK, Barbour S, Berger MJ, Bierman PJ, Bradbury B, Ellis G, Kirkegaard S, Kloth DD, Kris MG, Lim D, Michaud LB, Nabati L, Noonan K, Rugo HS, Siler D, Sorscher SM, Stelts S, Stucky-Marshall L, Todaro B, Urba SG. Antiemesis. J. Natl. Compr. Canc. Netw. 2012;10(4):456-85.

[39] Roila F, Herrstedt J, Aapro M, Gralla RJ, Einhorn LH, Ballatori E, Bria E, ClarkSnow RA, Espersen BT, Feyer P, Grunberg SM, Hesketh PJ, Jordan K, Kris MG, Maranzano E, Molassiotis A, Morrow G, Olver I, Rapoport BL, Rittenberg C, Saito M, Tonato M, Warr D. Guideline update for MASCC and ESMO in the prevention of chemotherapy- and radiotherapy-induced nausea and vomiting: results of the Perugia consensus conference. Ann. Oncol. 2010;21 Suppl 5:v23243.

[40] Jordan K, Gralla R, Jahn F, Molassiotis A. International antiemetic guidelines on chemotherapy induced nausea and vomiting (CINV): content and implementation 
in daily routine practice. Eur. J. Pharmacol. 2014;722:197-202.

[41] Jones JM, Qin R, Bardia A, Linquist B, Wolf S, Loprinzi CL. Antiemetics for chemotherapy-induced nausea and vomiting occurring despite prophylactic antiemetic therapy. J. Palliat. Med. 2011;14(7):810-4.

[42] Glaus A, Knipping C, Morant R, Böhme C, Lebert B, Beldermann F, Glawogger B, Ortega PF, Hüsler A, Deuson R. Chemotherapy-induced nausea and vomiting in routine practice: a European perspective. Support. Care Cancer. 2004;12(10):70815.

[43] Molassiotis A, Saunders MP, Valle J, Wilson G, Lorigan P, Wardley A, Levine E, Cowan R, Loncaster J, Rittenberg C. A prospective observational study of chemotherapy-related nausea and vomiting in routine practice in a UK cancer centre. Support. Care Cancer. 2008;16(2):201-8.

[44] Haiderali A, Menditto L, Good M, Teitelbaum A, Wegner J. Impact on daily functioning and indirect/direct costs associated with chemotherapy-induced nausea and vomiting (CINV) in a U.S. population. Support. Care Cancer. 2011;19(6):84351.

[45] Cohen L, de Moor CA, Eisenberg P, Ming EE, Hu H. Chemotherapy-induced nausea and vomiting: incidence and impact on patient quality of life at community oncology settings. Support. Care Cancer. 2007;15(5):497-503.

[46] Molassiotis A, Brearley SG, Stamataki Z. Use of antiemetics in the management of chemotherapy-related nausea and vomiting in current UK practice. Support. Care Cancer. 2011;19(7):949-56.

[47] Bloechl-Daum B, Deuson RR, Mavros P, Hansen M, Herrstedt J. Delayed nausea and vomiting continue to reduce patients' quality of life after highly and moderately emetogenic chemotherapy despite antiemetic treatment. J. Clin. Oncol. 2006;24(27):4472-8.

[48] Hilarius DL, Kloeg PH, van der Wall E, van den Heuvel JJG, Gundy CM, Aaronson NK. Chemotherapy-induced nausea and vomiting in daily clinical practice: a community hospital-based study. Support. Care Cancer. 2012;20(1):107-17.

[49] Lachaine J, Yelle L, Kaizer L, Dufour A, Hopkins S, Deuson R. Chemotherapy- 
induced emesis: quality of life and economic impact in the context of current practice in Canada. Support. Cancer Ther. 2005;2(3):181-7.

[50] Pirri C, Katris P, Trotter J, Bayliss E, Bennett R, Drummond P. Risk factors at pretreatment predicting treatment-induced nausea and vomiting in Australian cancer patients: a prospective, longitudinal, observational study. Support. Care Cancer. 2011;19(10):1549-63.

[51] Tina Shih Y-C, Xu Y, Elting LS. Costs of uncontrolled chemotherapy-induced nausea and vomiting among working-age cancer patients receiving highly or moderately emetogenic chemotherapy. Cancer. 2007;110(3):678-85.

[52] Ballatori E, Roila F, Ruggeri B, Porrozzi S, Iannopollo M, Soru G, Cruciani G, Daniele B, Locatelli MC, Pellissier J, Deuson R. The cost of chemotherapyinduced nausea and vomiting in Italy. Support. Care Cancer. 2007;15(1):31-8.

[53] Ihbe-Heffinger A, Ehlken B, Bernard R, Berger K, Peschel C, Eichler H-G, Deuson R, Thödtmann J, Lordick F. The impact of delayed chemotherapy-induced nausea and vomiting on patients, health resource utilization and costs in German cancer centers. Ann. Oncol. 2004;15(3):526-36.

[54] Viale PH, Grande C, Moore S. Efficacy and cost: avoiding undertreatment of chemotherapy-induced nausea and vomiting. Clin. J. Oncol. Nurs. 2012;16(4):E133-41.

[55] Yeh Y-C, McDonnell A, Klinger E, Fowler B, Matta L, Voit D, Reddy P. Comparison of healthcare resource use between patients receiving ondansetron or palonosetron as prophylaxis for chemotherapy-induced nausea and vomiting. J. Oncol. Pharm. Pract. 2011;17(3):179-85.

[56] Craver C, Gayle J, Balu S, Buchner D. Clinical and economic burden of chemotherapy-induced nausea and vomiting among patients with cancer in a hospital outpatient setting in the United States. J. Med. Econ. 2011;14(1):87-98.

[57] Nevidjon B, Chaudhary R. Controlling emesis: evolving challenges, novel strategies. J. Support. Oncol. 2010;8(4 Suppl 2):1-10.

[58] Schwartzberg LS. Chemotherapy-induced nausea and vomiting: clinician and patient perspectives. J. Support. Oncol. 2007;5(2 Suppl 1):5-12. 
[59] Richardson JL, Marks G, Levine A. The influence of symptoms of disease and side effects of treatment on compliance with cancer therapy. J. Clin. Oncol. 1988;6(11):1746-52.

[60] NCCN Clinical Practice Guidelines in Oncology: Antiemesis, Version 2.2015. National Comprehensive Cancer Network. Retrieved November 17, 2015, from http://www.nccn.org/professionals/physician_gls/pdf/antiemesis.pdf.

[61] Moher D, Liberati A, Tetzlaff J, Altman DG. Preferred reporting items for systematic reviews and meta-analyses: the PRISMA statement. BMJ. 2009;339:b2535.

[62] Kitazaki T, Fukuda Y, Fukahori S, Oyanagi K, Soda H, Nakamura Y, Kohno S. Usefulness of antiemetic therapy with aprepitant, palonosetron, and dexamethasone for lung cancer patients on cisplatin-based or carboplatin-based chemotherapy. Support. Care Cancer. 2014;23:185-90.

[63] Tamura K, Aiba K, Saeki T, Nakanishi Y, Kamura T, Baba H, Yoshida K, Yamamoto N, Kitagawa Y, Maehara Y, Shimokawa M, Hirata K, Kitajima M. Testing the effectiveness of antiemetic guidelines: results of a prospective registry by the CINV Study Group of Japan. Int. J. Clin. Oncol. 2015.

[64] Molassiotis A, Aapro M, Dicato M, Gascon P, Novoa SA, Isambert N, Burke TA, $\mathrm{Gu}$ A, Roila F. Evaluation of risk factors predicting chemotherapy-related nausea and vomiting: Results from a European prospective observational study. J. Pain Symptom Manage. 2014;47.

[65] Murakami M, Hashimoto H, Yamaguchi K, Yamaguchi I, Senba S, Siraishi T. Effectiveness of palonosetron for preventing delayed chemotherapy-induced nausea and vomiting following moderately emetogenic chemotherapy in patients with gastrointestinal cancer. Support. Care Cancer. 2014;22:905-9.

[66] Furukawa N, Akasaka J, Shigemitsu A, Sasaki Y, Nagai A, Kawaguchi R, Kobayashi H. Evaluation of the relation between patient characteristics and the state of chemotherapy-induced nausea and vomiting in patients with gynecologic cancer receiving paclitaxel and carboplatin. Arch. Gynecol. Obstet. 2014;289:85964.

[67] Celio L, Bonizzoni E, Bajetta E, Sebastiani S, Perrone T, Aapro MS. Palonosetron plus single-dose dexamethasone for the prevention of nausea and vomiting in 
women receiving anthracycline/cyclophosphamide-containing chemotherapy: Meta-analysis of individual patient data examining the effect of age on outcome in two phase I. Support. Care Cancer. 2013;21(2):565-73.

[68] Celio L, Frustaci S, Denaro A, Buonadonna A, Ardizzoia A, Piazza E, Fabi A, Capobianco AM, Isa L, Cavanna L, Bertolini A, Bichisao E, Bajetta E. Palonosetron in combination with 1-day versus 3-day dexamethasone for prevention of nausea and vomiting following moderately emetogenic chemotherapy: A randomized, multicenter, phase III trial. Support. Care Cancer. 2011;19:1217-25.

[69] Aapro M, Fabi A, Nolè F, Medici M, Steger G, Bachmann C, Roncoroni S, Roila F. Double-blind, randomised, controlled study of the efficacy and tolerability of palonosetron plus dexamethasone for 1 day with or without dexamethasone on days 2 and 3 in the prevention of nausea and vomiting induced by moderately emetogenic chemotherapy. Ann. Oncol. 2010;21:1083-8.

[70] Molassiotis A, Stamataki Z, Kontopantelis E. Development and preliminary validation of a risk prediction model for chemotherapy-related nausea and vomiting. Support. Care Cancer. 2013;21(10):2759-67.

[71] Sekine I, Segawa Y, Kubota K, Saeki T. Risk factors of chemotherapy-induced nausea and vomiting: Index for personalized antiemetic prophylaxis. Cancer Sci. 2013;104(6):711-7.

[72] Chan A, Tan SH, Low XH, Yap KY-L. Antiemetic effectiveness and nausea and vomiting incidence during capecitabine and oxaliplatin chemotherapy. Nurs. Res. 2012;61(6):405-12.

[73] Fleishman SB, Mahajan D, Rosenwald V, Nugent A V, Mirzoyev T. Prevalence of Delayed Nausea and/or Vomiting in Patients Treated With Oxaliplatin-Based Regimens for Colorectal Cancer. J. Oncol. Pract. 2012;8(3):136-40.

[74] Yap KY-L, Low XH, Chui WK, Chan A. Computational prediction of state anxiety in Asian patients with cancer susceptible to chemotherapy-induced nausea and vomiting. J. Clin. Psychopharmacol. 2012;32(2):207-17.

[75] Nakamura F, Higashi T. Pattern of prophylaxis administration for chemotherapyinduced nausea and vomiting: an analysis of city-based health insurance data. Int. J. Clin. Oncol. / Japan Soc. Clin. Oncol. 2012. 
[76] Celio L, Denaro A, Agustoni F, Bajetta E. Palonosetron Plus 1-Day Dexamethasone for the Prevention of Nausea and Vomiting Due to Moderately Emetogenic Chemotherapy: Effect of Established Risk Factors on Treatment Outcome in a Phase III Trial. J. Support. Oncol. 2012;10(2):65-71.

[77] Bourdeanu L, Frankel P, Yu W, Hendrix G, Pal S, Badr L, Somlo G, Luu T. Chemotherapy-induced nausea and vomiting in Asian women with breast cancer receiving anthracycline-based adjuvant chemotherapy. J. Support. Oncol. 2012;10(4):149-54.

[78] Warr DG, Street JC, Carides AD. Evaluation of risk factors predictive of nausea and vomiting with current standard-of-care antiemetic treatment: analysis of phase 3 trial of aprepitant in patients receiving adriamycin-cyclophosphamide-based chemotherapy. Support. Care Cancer. 2011;19(6):807-13.

[79] Warr DG, Hesketh PJ, Gralla RJ, Muss HB, Herrstedt J, Eisenberg PD, Raftopoulos H, Grunberg SM, Gabriel M, Rodgers A, Bohidar N, Klinger G, Hustad CM, Horgan KJ, Skobieranda F. Efficacy and tolerability of aprepitant for the prevention of chemotherapy-induced nausea and vomiting in patients with breast cancer after moderately emetogenic chemotherapy. J. Clin. Oncol. 2005;23:2822-30.

[80] Hassan BAR, Yusoff ZBM. Negative impact of chemotherapy on breast cancer patients QOL - utility of antiemetic treatment guidelines and the role of race. Asian Pac. J. Cancer Prev. 2010;11(6):1523-7.

[81] Hesketh PJ, Aapro M, Street JC, Carides AD. Evaluation of risk factors predictive of nausea and vomiting with current standard-of-care antiemetic treatment: analysis of two phase III trials of aprepitant in patients receiving cisplatin-based chemotherapy. Support. Care Cancer. 2010;18(9):1171-7.

[82] Poli-Bigelli S, Rodrigues-Pereira J, Carides AD, Ma GJ, Eldridge K, Hipple A, Evans JK, Horgan KJ, Lawson F. Addition of the neurokinin 1 receptor antagonist aprepitant to standard antiemetic therapy improves control of chemotherapyinduced nausea and vomiting: Results from a randomized, double-blind, placebocontrolled trial in Latin America. Cancer. 2003;97:3090-8.

[83] Hesketh PJ, Grunberg SM, Gralla RJ, Warr DG, Roila F, De Wit R, Chawla SP, Carides AD, Ianus J, Elmer ME, Evans JK, Beck K, Reines S, Horgan KJ. The oral 
neurokinin-1 antagonist aprepitant for the prevention of chemotherapy-induced nausea and vomiting: A multinational, randomized, double-blind, placebocontrolled trial in patients receiving high-dose cisplatin - The Aprepitant Protocol 052 Study G. J. Clin. Oncol. 2003;21:4112-9.

[84] Roscoe JA, Morrow GR, Colagiuri B, Heckler CE, Pudlo BD, Colman L, Hoelzer $\mathrm{K}$, Jacobs A. Insight in the prediction of chemotherapy-induced nausea. Support. Care Cancer. 2010;18(7):869-76.

[85] Shih V, Wan HS, Chan A. Clinical predictors of chemotherapy-induced nausea and vomiting in breast cancer patients receiving adjuvant doxorubicin and cyclophosphamide. Ann. Pharmacother. 2009;43(3):444-52.

[86] Dranitsaris G, Joy A, Young S, Clemons M, Callaghan W, Petrella T. Identifying patients at high risk for nausea and vomiting after chemotherapy: the development of a practical prediction tool. I. Acute nausea and vomiting. J Support Oncol. 2009;7(4):W1-8.

[87] Petrella T, Clemons M, Joy A, Young S, Callaghan W, Dranitsaris G. Identifying patients at high risk for nausea and vomiting after chemotherapy: the development of a practical validated prediction tool. II. Delayed nausea and vomiting. J Support Oncol. 2009;7(4):W9-16.

[88] Colagiuri B, Roscoe JA, Morrow GR, Atkins JN, Giguere JK, Colman LK. How do patient expectancies, quality of life, and postchemotherapy nausea interrelate? Cancer. 2008;113(3):654-61.

[89] Booth CM, Clemons M, Dranitsaris G, Joy A, Young S, Callaghan W, Trudeau M, Petrella T. Chemotherapy-induced nausea and vomiting in breast cancer patients: a prospective observational study. J. Support. Oncol. 2007;5(8):374-80.

[90] Roscoe JA, Bushunow P, Morrow GR, Hickok JT, Kuebler PJ, Jacobs A, Banerjee TK. Patient expectation is a strong predictor of severe nausea after chemotherapy: a University of Rochester Community Clinical Oncology Program study of patients with breast carcinoma. Cancer. 2004;101(11):2701-8.

[91] Liaw C-C, Chang H-K, Liau C-T, Huang J-S, Lin Y-C, Chen J-S. Reduced maintenance of complete protection from emesis for women during chemotherapy cycles. Am. J. Clin. Oncol. 2003;26(1):12-5. 
[92] Osoba D, Zee B, Pater J, Warr D, Latreille J, Kaizer L. Determinants of postchemotherapy nausea and vomiting in patients with cancer. Quality of Life and Symptom Control Committees of the National Cancer Institute of Canada Clinical Trials Group. J. Clin. Oncol. 1997;15(1):116-23.

[93] Hursti TJ, Avall-Lundqvist E, Börjeson S, Fredrikson M, Fürst CJ, Steineck G, Peterson C. Impact of tumour burden on chemotherapy-induced nausea and vomiting. Br. J. Cancer. 1996;74(7):1114-9.

[94] du Bois A, Meerpohl HG, Vach W, Kommoss FG, Fenzl E, Pfleiderer A. Course, patterns, and risk-factors for chemotherapy-induced emesis in cisplatin-pretreated patients: a study with ondansetron. Eur. J. Cancer. 1992;28(2-3):450-7.

[95] Pollera CF, Giannarelli D. Prognostic factors influencing cisplatin-induced emesis. Definition and validation of a predictive logistic model. Cancer. 1989;64(5):1117-22.

[96] Dranitsaris G, Bouganim N, Milano C, Vandermeer L, Dent S, Wheatley-Price P, Laporte J, Oxborough K-A, Clemons M. Prospective validation of a prediction tool for identifying patients at high risk for chemotherapy-induced nausea and vomiting. J. Support. Oncol. 2013;11(1):14-21.

[97] Oken MM, Creech RH, Tormey DC, Horton J, Davis TE, McFadden ET, Carbone PP. Toxicity and response criteria of the Eastern Cooperative Oncology Group. Am. J. Clin. Oncol. 1982;5(6):649-55.

[98] Higa GM, Auber ML, Hobbs G. Identification of a novel marker associated with risk for delayed chemotherapy-induced vomiting. Support. Care Cancer. 2012;20(11):2803-9.

[99] Aaronson NK, Ahmedzai S, Bergman B, Bullinger M, Cull A, Duez NJ, Filiberti A, Flechtner H, Fleishman SB, de Haes JC. The European Organization for Research and Treatment of Cancer QLQ-C30: a quality-of-life instrument for use in international clinical trials in oncology. J. Natl. Cancer Inst. 1993;85(5):365-76.

[100] McCorkle R, Young K. Development of a symptom distress scale. Cancer Nurs. 1978;1(5):373-8.

[101] Bouganim N, Dranitsaris G, Hopkins S, Vandermeer L, Godbout L, Dent S, Wheatley-Price P, Milano C, Clemons M. Prospective validation of risk prediction 
indexes for acute and delayed chemotherapy-induced nausea and vomiting. Curr. Oncol. 2012;19(6):e414-21.

[102] Harris PA, Taylor R, Thielke R, Payne J, Gonzalez N, Conde JG. Research electronic data capture (REDCap)--a metadata-driven methodology and workflow process for providing translational research informatics support. J. Biomed. Inform. 2009;42(2):377-81.

[103] Mosa ASM, Yoo I, Parker JC. Online Electronic Data Capture and Research Data Repository System for Clinical and Translational Research. Mo. Med. 2015;112(1):46-52.

[104] Marinov M, Mosa ASM, Yoo I, Boren SA. Data-mining technologies for diabetes: a systematic review. J. Diabetes Sci. Technol. 2011;5(6):1549-56.

[105] Quinlan JRR. Induction of decision trees. Mach. Learn. 1986;1(1):81-106.

[106] Quinlan JR. C4. 5: programs for machine learning. Morgan Kaufmann; 1993.

[107] Data Mining Tools See5 and C5.0. Retrieved November 21, 2015, from http://www.rulequest.com/see5-info.html.

[108] Breiman L, Friedman JH, Olshen RA, Stone CJ. Classification and Regression Trees. Wadsworth. Wadsworth International Group; 1984.

[109] Duda RO, Hart PE, Stork DG. Pattern Classification. 2nd ed. John Wiley \& Sons; 2001.

[110] ROSENBLATT F. The perceptron: a probabilistic model for information storage and organization in the brain. Psychol. Rev. 1958;65(6):386-408.

[111] Hall M, Frank E, Holmes G, Pfahringer B, Reutemann P, Witten IH. The WEKA data mining software: an update. ACM SIGKDD Explor. Newsl. 2009;11(1):10-8.

[112] R-Project. Retrieved November 20, 2015, from https://www.rproject.org/about.html.

[113] John G, Langley P. Estimating Continuous Distributions in Bayesian Classifiers. 
Proc. Elev. Conf. Uncertain. Artif. Intell., Morgan Kaufmann; 1995, pp. 338-45.

[114] Le Cessie S, Van Houwelingen JC. Ridge estimators in logistic regression. Appl. Stat. 1992:191-201.

[115] Freund Y, Schapire RE. Large margin classification using the perceptron algorithm. Mach. Learn. 1999;37(3):277-96.

[116] Platt J, others. Fast training of support vector machines using sequential minimal optimization. Adv. Kernel Methods support Vector Learn. 1999;3.

[117] Wedyan S. Review and Comparison of Associative Classification Data Mining Approaches. Int. J. Comput. Information, Syst. Control Eng. 2014;8(1):34-45.

[118] Liu B, Hsu W, Ma Y. Integrating classification and association rule mining. Proc. fourth Int. Conf. Knowl. Discov. data Min., 1998.

[119] Wenmin Li, Jiawei Han, Jian Pei. CMAR: accurate and efficient classification based on multiple class-association rules. Proc. 2001 IEEE Int. Conf. Data Min., IEEE Comput. Soc; 2001, pp. 369-76.

[120] Antonie M-L, Zaiane OR. Text document categorization by term association. 2002 IEEE Int. Conf. Data Mining, 2002. Proceedings., IEEE Comput. Soc; 2002, pp. 19-26.

[121] Yin X, Han J. CPAR: Classification based on Predictive Association Rules. Proc. 2003 SIAM Int. Conf. Data Min., Philadelphia, PA: Society for Industrial and Applied Mathematics; 2003, pp. 331-5.

[122] Xiaoyuan Xu, Guoqiang Han, Huaqing Min. A novel algorithm for associative classification of image blocks. Fourth Int. Conf. onComputer Inf. Technol. 2004. CIT '04., IEEE; 2004, pp. 46-51.

[123] Antonie M-L, Zaïane OR. An Associative Classifier Based on Positive and Negative Rules. Proc. 9th ACM SIGMOD Work. Res. Issues Data Min. Knowl. Discov., New York, NY, USA: ACM; 2004, pp. 64-9.

[124] Baralis E, Garza P. A lazy approach to pruning classification rules. 2002 IEEE Int. 
Conf. Data Mining, 2002. Proceedings., IEEE Comput. Soc; 2002, pp. 35-42.

[125] Thabtah FA, Cowling P, Yonghong Peng. MMAC: A New Multi-Class, MultiLabel Associative Classification Approach. Fourth IEEE Int. Conf. Data Min., IEEE; 2004, pp. 217-24.

[126] Thabtah F, Cowling P, Peng Y. MCAR: multi-class classification based on association rule. Comput. Syst. Appl. 2005. 3rd ACS/IEEE Int. Conf., 2005, p. 33.

[127] Tang Z, Liao Q. A New Class Based Associative Classification Algorithm. IMECS. 2007;2007:685-9.

[128] Li X, Qin D, Yu C. ACCF: Associative Classification Based on Closed Frequent Itemsets. 2008 Fifth Int. Conf. Fuzzy Syst. Knowl. Discov., IEEE; 2008, pp. 3804.

[129] Kundu G, Islam MM, Munir S. ACN: An associative classifier with negative rules. 2008 IEEE Int. Conf. Syst. Syst. Eng., IEEE; 2008, pp. 1-6.

[130] Niu Q, Xia S-X, Zhang L. Association Classification Based on Compactness of Rules. 2009 Second Int. Work. Knowl. Discov. Data Min., IEEE; 2009, pp. 245-7.

[131] Wang X, Yue K, Niu W, Shi Z. An approach for adaptive associative classification. Expert Syst. Appl. 2011;38(9):11873-83.

[132] Yusof Y, Refai MH. MMCAR: Modified multi-class classification based on association rule. 2012 Int. Conf. Inf. Retr. Knowl. Manag., IEEE; 2012, pp. 6-11.

[133] Agrawal R, Imielinski T, Swami A. Mining association rules between sets of items in large databases. ACM SIGMOD Rec. 1993;22(2):207-16.

[134] Agrawal R, Srikant R. Fast algorithms for mining association rules. Proc. 20th Int. Conf. Very Large Data Bases, VLDB, vol. 1215, San Francisco, CA, USA: Morgan Kaufmann Publishers Inc.; 1994, pp. 487-99.

[135] Yoo I, Alafaireet P, Marinov M, Pena-Hernandez K, Gopidi R, Chang J-F, Hua L. Data mining in healthcare and biomedicine: a survey of the literature. J. Med. Syst. 2012;36(4):2431-48. 
[136] Wong PC, Whitney P, Thomas J. Visualizing association rules for text mining. 1999 IEEE Symp. Inf. Vis. (InfoVis '99), San Francisco, California: IEEE Comput. Soc; 1999, pp. 120-3,.

[137] Dimitrijević M, Bošnjak Z, Subotica S. Discovering interesting association rules in the web log usage data. Interdiscip. J. Information, Knowledge, Manag. 2010;5:191-207.

[138] Agrawal R, Imieliński T, Swami A. Mining association rules between sets of items in large databases. Proc. 1993 ACM SIGMOD Int. Conf. Manag. data SIGMOD '93, New York, New York, USA: ACM Press; 1993, pp. 207-16.

[139] Han J, Pei J, Yin Y. Mining frequent patterns without candidate generation. ACM SIGMOD Rec. 2000;29(2):1-12.

[140] Hahsler M, Grün B, Hornik K. arules-A computational environment for mining association rules and frequent item sets. J. Stat. Softw. 2005;14(15).

[141] Hornik K. Frequently Asked Questions on R. Retrieved March 8, 2013, from http://cran.r-project.org/doc/FAQ/R-FAQ.html.

[142] Han J, Pei J, Yin Y. Mining frequent patterns without candidate generation: A frequent-pattern tree approach. Data Min. Knowl. Discov. 2004;8:53-87.

[143] Han J, Pei J. Mining frequent patterns without candidate generation. ACM SIGMOD Rec. 2000;29(2):1-12.

[144] Package "arules." Retrieved March 8, 2013, from http://cran.rproject.org/web/packages/arules/arules.pdf.

[145] Attribute-Relation File Format (ARFF). Retrieved March 8, 2011, from http://www.cs.waikato.ac.nz/ml/weka/arff.html.

[146] Chen T-J, Chou L-F, Hwang S-J. Application of a data-mining technique to analyze coprescription patterns for antacids in Taiwan. Clin. Ther. 2003;25(9):2453-63. 
[147] Yoo I, Mosa ASM. Analysis of PubMed User Sessions Using a Full-Day PubMed Query Log: A Comparison of Experienced and Nonexperienced PubMed Users. JMIR Med. Informatics. 2015;3:e25.

[148] Mosa ASM, Yoo I. A study on PubMed search tag usage pattern: association rule mining of a full-day PubMed query log. BMC Med. Inform. Decis. Mak.

2013;13(January):8.

[149] Mosa ASM, Yoo I. Association mining of search tags in PubMed search sessions. 2014 IEEE Int. Conf. Bioinforma. Biomed., IEEE; 2014, pp. 56-61.

[150] Herskovic JR, Tanaka LY, Hersh W, Bernstam E V. A day in the life of PubMed: analysis of a typical day's query log. J. Am. Med. Inform. Assoc. 2007;14(2):21220.

[151] Zaki M, Parthasarathy S, Ogihara M, Li W. New algorithms for fast discovery of association rules. 3rd Intl. Conf. Knowl. Discov. Data Min., vol. 20, 1997, pp. 283-6.

[152] Association rules Viewer. Retrieved March 9, 2013, from http://www.lifl.fr/ jourdan/download/arv.html.

[153] Mosa ASM, Yoo I. Association mining of search tags in PubMed search sessions. 2014 Int. Work. Biomed. Heal. Informatics conjunction with IEEE Int. Conf. Bioinforma. Biomed., Belfast, UK: 2014.

[154] NCCN Clinical Practice Guidelines in Oncology: Antiemesis, Version 1.2013. National Comprehensive Cancer Network. Retrieved February 7, 2013, from http://www.nccn.org/professionals/physician_gls/pdf/antiemesis.pdf. 


\section{VITA}

Abu Mosa is a health informatics doctoral student at the MU Informatics Institute (MUII). He has gained BS in Computer Science from the Bangladesh University of Engineering and Technology and MS in Computer Science from the University College Dublin, Ireland. His research interest spans in the area of medical informatics focusing on the development of technologies for healthcare. He has co-authored several journal articles and a book chapter. He has served as the reviewer of many leading medical informatics journals and conferences. He has demonstrated superlative leadership skills during his time here at MU; he served as the Treasurer and President of MUII Graduate Student Association and Chair of the 2012 Missouri Informatics Symposium. In his doctoral dissertation project, he applied data mining techniques for the development of a novel, precise and dynamic prediction engine for practicing precision and personalized medicine to prevent chemotherapy-induced nausea and vomiting (CINV). 